\title{
One-dimensional Fermi liquids
}

\author{
Johannes Voit \\ Bayreuther Institut für Makromolekülforschung (BIMF) \\ and Theoretische Physik 1 \\ Universität Bayreuth \\ D-95440 Bayreuth (Germany)由 \\ and Institut Laue-Langevin \\ F-38042 Grenoble (France)
}

submitted to Reports on Progress in Physics on

November 19, 1994

last revision and update on

February 1, 2008

\footnotetext{
${ }^{1}$ Present address
} 


\section{Abstract}

We review the progress in the theory of one-dimensional (1D) Fermi liquids which has occurred over the past decade. The usual Fermi liquid theory based on a quasi-particle picture, breaks down in one dimension because of the Peierls divergence in the particlehole bubble producing anomalous dimensions of operators, and because of charge-spin separation. Both are related to the importance of scattering processes transferring finite momentum. A description of the low-energy properties of gapless one-dimensional quantum systems can be based on the exactly solvable Luttinger model which incorporates these features, and whose correlation functions can be calculated. Special properties of the eigenvalue spectrum, parameterized by one renormalized velocity and one effective coupling constant per degree of freedom fully describe the physics of this model. Other gapless 1D models share these properties in a low-energy subspace. The concept of a "Luttinger liquid" implies that their low-energy properties are described by an effective Luttinger model, and constitutes the universality class of these quantum systems. Once the mapping on the Luttinger model is achieved, one has an asymptotically exact solution of the 1D many-body problem. Lattice models identified as Luttinger liquids include the 1D Hubbard model off half-filling, and variants such as the $t-J$ - or the extended Hubbard model. Also 1D electron-phonon systems or metals with impurities can be Luttinger liquids, as well as the edge states in the quantum Hall effect.

We discuss in detail various solutions of the Luttinger model which emphasize different aspects of the physics of 1D Fermi liquids. Correlation functions are calculated in detail using bosonization, and the relation of this method to other approaches is discussed. The correlation functions decay as non-universal power-laws, and scaling relations between their exponents are parameterized by the effective coupling constant. Charge-spin separation only shows up in dynamical correlations. The Luttinger liquid concept is developed from perturbations of the Luttinger model. Mainly specializing to the 1D Hubbard model, we review a variety of mappings for complicated models of interacting electrons onto Luttinger models, and thereby obtain their correlation functions. We also discuss the generic behaviour of systems not falling into the Luttinger liquid universality class because of gaps in their low-energy spectrum. The Mott transition provides an example for the transition from Luttinger to non-Luttinger behaviour, and recent results on this problem are summarized. Coupling chains by interactions or tunneling allows transverse coherence to establish in the single- or two-particle dynamics, and drives the systems away from a Luttinger liquid. We discuss the influence of charge-spin separation and of the anomalous dimensions on the transverse dynamics of the electrons. The edge states in the quan- 
tum Hall effect provide a realization of a modified, chiral Luttinger liquid whose detailed properties differ from those of the standard model. The article closes with a summary of experiments which can be interpreted in favour of Luttinger liquid-correlations in the "normal" state of quasi-1D organic conductors and superconductors, charge density wave systems, and semiconductors in the quantum Hall regime. 


\section{Contents}

$\begin{array}{llr}1 & \text { Introduction } & 1\end{array}$

1.1 Motivation . . . . . . . . . . . . . . . . . . . . 1

1.2 Purpose and structure of this review . . . . . . . . . . . . . . . 5

\begin{tabular}{|lll}
2 & Fermi liquid theory and its failure in one dimension & 8
\end{tabular}

2.1 The Fermi liquid . . . . . . . . . . . . . . . . . . . . . . . . . . . . 8

2.2 Breakdown of Fermi liquid theory in one dimension . . . . . . . . . . . . . 10

\begin{tabular}{|lll}
\hline 3 & The Luttinger model & 14
\end{tabular}

3.1 Low-energy phenomenology in 1D - the Luttinger model . . . . . . . . . . 14

3.1.1 Ground state and elementary excitations of 1D fermions . . . . . . 14

3.1 .2 Tomonaga-Luttinger Hamiltonian . . . . . . . . . . . . . . . . 15

3.1 .3 Symmetries and conservation laws . . . . . . . . . . . . . . . . . . . 16

3.2 Boson solution of the Luttinger model $\ldots \ldots \ldots$. . . . . . . . . . . . 18

3.2 .1 Diagonalization of Hamiltonian . . . . . . . . . . . . . . . . . . . . 19

3.2 .2 Bosonization . . . . . . . . . . . . . . . . . . 24

3.3 Physical Properties of the Luttinger Model - Thermodynamics and Corre-

lation Functions . . . . . . . . . . . . . . . . . . . . . 27

3.3 .1 Thermodynamics and transport . . . . . . . . . . . . . . . . . . . . 28

3.3 .2 Single- and two-particle correlation functions . . . . . . . . . . . . . 30

3.4 Dynamical correlations: the spectral properties of Luttinger liquids . . . . 36

3.5 Alternative methods . . . . . . . . . . . . . . . . . . 38

3.5 .1 Green function methods . . . . . . . . . . . . . . . . . . 38

3.5 .2 Other bosonic schemes . . . . . . . . . . . . . . . . . . 42

3.6 Conformal field theory and bosonization . . . . . . . . . . . . . . 43

3.6 .1 Conformal invariance at a critical point . . . . . . . . . . . . . . 43

3.6 .2 The Gaussian mode . . . . . . . . . . . . . . . . . . . . . . . . 50

\begin{tabular}{|lll}
4 The Luttinger Liquid & 56
\end{tabular}

4.1 The conjecture . . . . . . . . . . . . . . . . . . 56

4.2 Luttinger model with nonlinear dispersion - the emergence of higher harmonics . . . . . . . . . . . . . . . . . . . 58

4.3 Backward and Umklapp scattering . . . . . . . . . . . . . . . . . . . . 59

4.4 Lattice models: Hubbard \& Co . . . . . . . . . . . . . . . . . . . . . . . . 64 
4.4 .1 Models . . . . . . . . . . . . . . . . . . . . . . . . . 64

4.4 .2 Bethe Ansatz . . . . . . . . . . . . . . . . . . . . . . . 65

4.4 .3 Low-energy properties of one-dimensional lattice models . . . . . . 69

4.5 Electron-phonon interaction and impurity scattering . . . . . . . . . . . . . 82

4.6 Transport in Luttinger liquids . . . . . . . . . . . . . . . . . . . . . . 86

4.6 .1 Electron-electron scattering . . . . . . . . . . . . . . . . 86

4.6 .2 Electron-impurity scattering . . . . . . . . . . . . . . . . . . 88

4.6 .3 Electron-phonon scattering . . . . . . . . . . . . . . . . . . 94

4.7 The notion of a Landau-Luttinger liquid . . . . . . . . . . . . . . . . . . . . . 95

5 Alternatives to the Luttinger liquid: spin gaps, the Mott transition, and $\begin{array}{ll}\text { phase separation } & 98\end{array}$

5.1 Spin gaps . . . . . . . . . . . . . . . . . . . . . . . . . . . . 98

5.2 The Mott transition . . . . . . . . . . . . . . . . . . . . . . . . 100

5.3 Phase separation . . . . . . . . . . . . . . . . . . 107

$\begin{array}{lll}6 & \text { Extensions of the Luttinger Liquid } & 109\end{array}$

6.1 Multi-component models . . . . . . . . . . . . . . . . . . . . 110

6.2 Crossover to higher dimensions . . . . . . . . . . . . . . . . . . 114

6.3 Edge states in the quantum Hall effect . . . . . . . . . . . . . . . . 128

7 The normal state of quasi-one-dimensional metals - a Luttinger liquid? 134

7.1 Organic conductors and superconductors . . . . . . . . . . . . . . . . . . 134

7.2 Inorganic charge density wave materials . . . . . . . . . . . . . . . . . 138

7.3 Semiconductor heterostructures . . . . . . . . . . . . . . . . . . . . . 139

$\begin{array}{lll}8 & \text { Summary } & 141\end{array}$ 


\section{Chapter 1}

\section{Introduction}

\subsection{Motivation}

Strongly correlated fermions are an important problem in solid state physics. Over the last one or two decades, experiments on many classes of materials have provided evidence that strong correlations are a central ingredient for the understanding of their physical properties. Among them are the heavy fermion compounds, the high- $T_{c}$ superconductors, a variety of intimately related organic metals, superconductors, and insulators, just to name a few. Also in normal metals, the interactions between the electrons are rather strong, although the correlations may be much weaker than in the systems mentioned before. The effective dimension of the electron gas plays an important role in correlating interacting fermions, and the materials listed are essentially three-(3D), two-(2D), and one-dimensional (1D), respectively. Correlations are also very important in semiconductor heterostructures and quantum wires, being two- or one-dimensional, including the Quantum Hall regime.

The theoretical description of strongly interacting electrons poses a formidable problem. Exact solutions of specific models usually are impossible, exception made for certain one-dimensional models to be discussed later. Fortunately, such exact solutions are rarely required (and more rarely even practical) when comparing with experiment. Most measurements, in fact, only probe correlations on energy scales small compared to the Fermi energy $E_{F}$ so that only the low-energy sector of a given model is of importance. Moreover, only at low energies can we hope to excite only a few degrees of freedom, for which a meaningful comparison to theoretical predictions can be attempted.

Correlated fermions in three dimensions are a well studied problem. Their theoretical description, by Fermi liquid theory, is approximate but well understood [1, 2]. It becomes an asymptotically exact solution for low energies and small wavevectors $\left(E \rightarrow E_{F},|\mathbf{k}| \rightarrow k_{F}, T \rightarrow 0\right)$. The limitation to low energies is instrumental here because, together with Fermi statistics, it implies that the phase space for excitations is severely restricted. In one dimension, there is a variety of exactly solvable models, which have been known for quite a time, but a deeper understanding of their mutual relationships and their relevance for describing the generic low-energy physics, close to the 1D 
Fermi surface, has emerged only rather recently. These relations as well as the properties of such one-dimensional Fermi liquids, or following Haldane "Luttinger liquids" [3], are the main subjects of this review article. Here we shall use the terms "one-dimensional Fermi liquids" and "Luttinger liquids" synonymously, although, as we show below, Fermi liquid behaviour as it is established in $3 \mathrm{D}$ is not possible in $1 \mathrm{D}$.

Fermi liquid theory is based on (but not exhausted by) a picture of quasi-particles evolving out of the particles (holes) of a Fermi gas upon adiabatically switching on interactions [1, 2]. They are in one-to-one correspondence with the bare particles and, specifically, carry the same quantum numbers and obey Fermi-Dirac statistics. The free Fermi gas thus is the solvable model on which Fermi liquid theory is built. The electronelectron interaction has three main effects: (i) it renormalizes the kinematic parameters of the quasi-particles such as the effective mass, and the thermodynamic properties (specific heat, susceptibility), described by the Landau parameters $F_{n}^{a, s}$; (ii) it gives them a finite lifetime diverging, however, as $\tau \sim\left(E-E_{F}\right)^{-2}$ as the Fermi surface is approached, so that the quasi-particles are robust against small displacements away from $E_{F}$; (iii) it introduces new collective modes. The existence of quasi-particles formally shows up through a finite jump $z_{k_{F}}$ of the momentum distribution function $n(k)$ at the Fermi surface, corresponding to a finite residue of the quasi-particle pole in the electron's Green function.

One-dimensional Fermi liquids are very special in that they retain a Fermi surface (if defined as the set of points where the momentum distribution or its derivatives have singularities) enclosing the same $k$-space volume as that of free fermions, in agreement with Luttinger's theorem [4]. However, there are no fermionic quasi-particles, and their elementary excitations are rather bosonic collective charge and spin fluctuations dispersing with different velocities. An incoming electron decays into such charge and spin excitations which then spatially separate with time (charge-spin separation). The correlations between these excitations are anomalous and show up as interaction-dependent nonuniversal power-laws in many physical quantities where those of ordinary metals are characterized by universal (interaction independent) powers.

To be more specific, a list of salient properties of such 1D Fermi liquids includes: (i) a continuous momentum distribution function $n(k)$, varying with as $\left|k-k_{F}\right|^{\alpha}$ with an interaction-dependent exponent $\alpha$, and a pseudogap in the single-particle density of states $\propto|\omega|^{\alpha}$, consequences of the non-existence of fermionic quasi-particles (the quasi-particle residue vanishes as $z_{k} \sim\left|k-k_{F}\right|^{\alpha}$ as $k \rightarrow k_{F}$ ); (ii) similar power-law behaviour in all correlation functions, specifically in those for superconducting and spin or charge density wave fluctuations, with universal scaling relations between the different nonuniversal exponents, which depend only on one effective coupling constant per degree of freedom; (iii) finite spin and charge response at small wavevectors, and a finite Drude weight in the conductivity; (iv) charge-spin separation; (v) persistent currents quantized in units of $2 k_{F}$. All these properties can be described in terms of only two effective parameters per degree of freedom which take over in 1D the role of the Landau parameters familiar from Fermi liquid theory.

The reasons for these peculiar properties are found in the very special Fermi surface topology of 1D fermions producing both singular particle-hole response and severe conser- 
vation laws. In a $1 \mathrm{D}$ chain, one has simply two Fermi "points" $\pm k_{F}$, and the Fermi surface of an array of chains consists of two parallel sheets (in the absence of interchain hopping). In both cases, one has perfect nesting, namely one complete Fermi sheet can be translated onto the other by a single wavevector $\pm 2 k_{F}$. This produces a singular particle-hole response at $2 k_{F}$, the well-known Peierls instability [5]. This type of response is assumed finite in Fermi liquid theory but, in 1D, is divergent for repulsive forward scattering (or attractive backscattering, the case considered by Peierls), leading to a breakdown of the Fermi liquid description. In addition, we have, as in 3D, the BCS singularity for attractive interactions [6]. On the other hand, the disjoint Fermi surface gives a well-defined dispersion, i.e. particle-like character, to the low-energy particle-hole excitations. They now can be taken as the building blocks upon which to construct a description of the 1D low-energy physics.

These properties are generic for one-dimensional Fermi liquids but particularly prominent in a 1D model of interacting fermions proposed by Luttinger [7] and Tomonaga [8] and solved exactly by Mattis and Lieb [9]. All correlation functions of the Luttinger model can be computed exactly, so that one has direct access to all physical properties of interest. The notion of a "Luttinger liquid" was coined by Haldane to describe these universal low-energy properties of gapless 1D quantum systems, and to emphasize that an asymptotic $(\omega \rightarrow 0, q \rightarrow 0)$ description can be based on the Luttinger model in much the same way as the Fermi liquid theory in 3D is based on the free Fermi gas. The basic ideas and procedures had been discussed earlier by Efetov and Larkin [10] but passed largely unnoticed. The name "Tomonaga - Luttinger liquid" might be more appropriate to give credit to Tomonaga's important early contribution but has not become widely popular today.

Despite this apparently very different set of physical properties, there are also similarities in the structures of Fermi and Luttinger liquids. Some concepts make these similarities particularly apparent: conformal field theory, where we essentially exploit the fact that both Fermi and Luttinger liquids (the former in 1D, of course) are critical, in the language of the theory of phase transitions, and possess the same central charge; a description of both theories based on Ward identities (i.e. symmetries and conservation laws), and the notion of a "Landau-Luttinger liquid", where one formulates a Fermi liquid picture for the pseudo-particles appearing in the exact Bethe-Ansatz solution of models like the 1D Hubbard model. Other methods, often more suitable for the practical calculations required by a solid state physicist, like bosonization, more strongly emphasize the differences between Fermi and Luttinger liquids.

In two dimensions, the applicability of Fermi liquid theory, specifically to the high- $T_{c}$ problem, is quite controversial. In fact, much of the recent interest in Luttinger liquids is due to Anderson's observation that the normal state properties of the $2 \mathrm{D}$ high- $T_{c}$ superconductors are strikingly different from all known metals and cannot be reconciled with Fermi-liquid theory; they are more similar to properties of 1D models [11. Anderson proposed that the essential physics be contained in the 2D Hubbard model and suggested a picture of a "tomographic Luttinger liquid" for the ground state and the low-energy excitations of this model, building on Haldane's earlier work in 1D, to give 
a more systematic basis to these conjectured non-Fermi liquid properties of the high- $T_{c}$ superconductors. Arguments have been advanced, however, also in favour of Fermi-liquid physics [12]. In addition a theory somewhat intermediate between Fermi and Luttinger liquids, a "marginal Fermi liquid" has been proposed [13], where the quasi-particle residue $z_{k}$ vanishes logarithmically as $k \rightarrow k_{F}$. (We parenthetically note that there is no simple solvable model, like the Fermi gas, or the Luttinger model, onto which one could build the marginal Fermi liquid phenomenology [13]. Very recent work seems to indicate, however, that certain impurity models do produce marginal Fermi liquid behaviour [14].)

While the relevance of Anderson's ideas is still quite controversial and no unambiguous formal justification has been published to date, they have refocussed attention on 1D models as paradigms for the breakdown of Fermi-liquid theory: there are few other instances where this has been established firmly. The main progress of the last years, to be reviewed here, is related to the realization that, in 1D, a variety of models allows essentially exact calculations of the physical properties of "exotic" non-Fermi-liquid metals. Emphasis has been directed in two main directions. (i) The relation of models defined on a lattice, such as the 1D Hubbard model, to continuum theories of the Tomonaga-Luttinger type. There had been a widespread opinion, that the lattice models would be appropriate to model the limit of strong electron-electron interactions, while the field theories would be better suited for weak-coupling situations. It has now become clear that this is not so, and that the continuum theories rather are the asymptotic low-energy limits of the lattice models even at arbitrarily strong coupling. Moreover, this mapping has provided us with several algorithms to extract the effective parameters of the continuum models from the (either Bethe Ansatz or numerical) solution of the lattice models. It therefore provides an asymptotically exact solution to the 1D many-body problem. We now can compute essentially all correlation functions for lattice models, an impossible task if one wanted to use the lattice solution directly. (ii) The calculation of physical properties from the now known correlation function allows to work out the distinctive difference of such Luttinger liquids from the predictions of Fermi liquid theory in higher dimensions, so as to get tools for the diagnosis of non-Fermi liquid behaviour.

With the general excitement in the community over the spectacular physics of the high$T_{c}$ superconductors, it has been somewhat forgotten that there are many families of organic and inorganic quasi-1D metals [15, 16] which do deviate strikingly from Fermi-liquid behaviour (at least from ordinary metals) in their normal state, and undergo a variety of low-temperature phase transitions into, e. g., charge or spin density wave (CDW/SDW) insulating phases or even become superconducting. The normal state properties of these materials are often highly anisotropic and justify application of 1D theory. We therefore possess a laboratory playground where we can confront theoretical evaluations of the distinctive properties of such "Luttinger liquids" with experimental reality - in a situation where the theoretical basis (namely one-dimensionality) is quite firmly established from experiment.

There is thus at least a threefold motivation to study models of $1 \mathrm{D}$ interacting electrons: (i) The search for a coherent description of the quasi-1D metals whose "exotic" properties have been studied over nearly two decades and which continue to be the focus 
of intense experimental efforts. (ii) 1D models as a paradigm for "metallic" systems which are not Fermi liquids. The detailed calculations possible here will hopefully sharpen our understanding of critical requirements for the breakdown of Fermi-liquid theory in general, and how such scenarios translate into experimental reality. There are only a few established examples of non-Fermi liquid metals in higher dimension such as the multichannel Kondo problem, but even these reduce, due to the spherical symmetry commonly assumed, to effective 1D problems [17]. (iii) The possibility of finding exact solutions to nontrivial many-body problems.

\subsection{Purpose and structure of this review}

This article will present a practical introduction to Luttinger liquids. It attempts to combine a review of the progress of the last couple of years with a self-contained and pedagogical presentation of the Luttinger model, its solution, and its properties (i.e. correlation functions) and especially emphasize bosonization as a simple practical means both to solve the model and to calculate correlation functions. Based on this, we carry on to the notion of a Luttinger liquid and a discussion of the various methods employed to map a complicated 1D many-body problem onto the relatively simple Luttinger model. For all models to be discussed, the emphasis will be on their properties, i.e. correlation functions, which ultimately can be compared with experiment. I also hope to demonstrate that the Luttinger liquid often is a useful device if none of the exact nonperturbative methods to compute correlation exponents works: incorporating all essential features of the 1D Fermi liquid, it is presumably the best possible starting point for a renormalization group analysis of the problem at hand.

On the other hand, we shall be quite schematic concerning the methods used to achieve an exact solution of the (lattice) models we are interested in, such as the Bethe Ansatz or numerical diagonalization techniques. We shall be more concerned with the various methods which have been invented to extract effective Luttinger parameters given a certain type of solution of the starting models, to compare their virtues and drawbacks and emphasize their complementarity. Moreover, we do not attempt to present a complete overview of the numerous 1D models used to describe strongly correlated electrons. Rather, we shall concentrate on a few of them, most often the paradigmatic Hubbard model. The methods discussed often can be applied without significant changes to other models the reader might be more interested in.

The field of 1D Fermi liquids is not new. Stimulated by and stimulating the research on quasi-1D organic conductors in the seventies and early eighties, a number of useful review articles has been available for some time. The meanwhile classical reviews of Sólyom 18 and Emery [19] contain much material on the use of renormalization group (with respect to a 1D Fermi gas) to treat the singularities generated perturbatively by the 1D interactions. There is also material on the solution of the Tomonaga-Luttinger model either by bosonization (in a somewhat approximate but for many purposes sufficient form) and through the use of Ward identities. We are extremely brief on papers duely covered there. 
Our presentation here will be limited to abelian bosonization. Nonabelian bosonization, retaining manifestly the $S U(2)$-invariance in the spin sector, is reviewed by Affleck [20]. Firsov, Prigodin, and Seidel [21] and Bourbonnais and Caron [22] more strongly emphasize phase transitions in real materials made of coupled 1D chains, and especially the paper by Bourbonnais and Caron gives a very modern presentation combining functional integral representations with renormalization group. A classical review on the earlier work on organic conductors is by Jérôme and Schulz [15] and the more recent developments have been summarized by Jérôme [23] and Williams et al. [24]. A pedagogical overview of both experimental and theoretical aspects can be found in the Proceedings of the 1986 NATOASI in Magog (Canada) [25] while the latest progress on organic materials is collected in the Proceedings of the biannual Synthetic Metals conferences [16]. Some subjects do not receive due coverage in this article: concerning the Bethe Ansatz, there are reviews by Sutherland [26], Korepin et al. [27], and Izyumov and Skryabin [28], and there is also a vast literature on conformal field theory [29]. Other problems of high current interest, and intimately related to our subject, could not be included for restrictions in space and time: spin chains, which can be related by a variety of methods to 1D interacting fermions; all developments starting from the Calogero-Sutherland model; one-dimensional bosons; persistent currents in mesoscopic rings, where interesting contributions originate from the study of 1D fermions despite the 3D spherical Fermi surface in the real materials, and many more. We hope that others take up the challenge to review these active areas.

This article is structured as follows. Chapter 2 will complement this brief introduction in that it discusses the breakdown of the Fermi liquid on a more technical level and identifies the relevant features of one-dimensionality. Specifically we show (i) how the Peierls divergence produces an instability of the 1D Fermi gas in the presence of repulsive interactions, (ii) how the breakdown of a quasi-particle picture can be seen in a secondorder perturbation calculation, and (iii) where Landau's derivation of a transport equation crashes in 1D.

Chapter 3 will present a detailed discussion of the Luttinger model from various angles. In Section 3.1, we argue that a universal low-energy description of 1D Fermi liquids can be based on this model. We define the Hamiltonian and discuss its symmetries and conservation laws which are essential for all solutions. In Section 3.2.1, we give a solution using a boson representation of the Hamiltonian, before constructing an explicit operator identity between fermions and these bosons in Section 3.2.2. This representation is fruitfully employed in Section 3.3 for a calculation of the Luttinger model correlation functions. The manifestation of charge-spin separation in dynamical correlations is the subject of Section 3.4. An alternative method of solution based on the equations of motion of the Green functions, and on Ward identities, is presented in Section 3.5. Another alternative for constructing a boson representation of the fermions, conformal field theory, is introduced in Section 3.6 and shown to be fully equivalent to the operator approach of the earlier sections.

The Luttinger model is based on very strong restrictions on the dispersion and interaction of the particles. In Chapter 4 we shall pass beyond these restrictions and show that the Luttinger physics still is conserved in a low-energy subspace of more realistic 
models. This is conjectured in Section 4.1, and then case studies are presented to its support. With nonlinear band dispersion (Section 4.2), the fermion operators acquire higher harmonics in $k_{F}$. Large-momentum transfer scattering in (Section 4.3) lifts unrealistic degeneracies of Luttinger model correlation functions by logarithmic corrections. The correlations of various lattice models are evaluated in Section 4.4. Electron-phonon systems or dirty 1D metals can also have Luttinger liquid correlations, and we touch upon these problems in Section 4.5. Section 4.6 shows that rich transport phenomena occur as one goes away from the simple Luttinger model. Finally, in Section 4.7, we show that the low-energy physics of the 1D Hubbard model is determined by low-energy excitations in the charge-momentum- and spin-rapidity-distribution functions, and that in each sector, one can therefore formulate a Fermi-liquid theory for its excitations.

Not all 1D systems are Luttinger liquids. In some cases, there are gaps in either the charge or spin excitation spectra, and phase separation can occur (at least in models). Chapter 5 discusses these cases. We expand especially on the Mott transition in Section 5.2 which has been studied in considerable detail during the past years.

In Chapter 6, we go beyond the framework of the Luttinger liquid outlined before. We discuss multi-band models in Section 6.1. An important issue has been the crossover from the 1D Luttinger liquid to higher-dimensional behaviour. We elaborate on this problem in Section 6.2. Finally, we describe the modelling of edge excitations supporting the transport in the quantum Hall effect in terms of a chiral Luttinger liquid in Section 6.3. While the general features are similar to the standard Luttinger liquid, the edge excitations have irrational charges and constitute a new universality class, described by a conformal field theory with central charge $c \neq 1$.

This review closes with a summary of experiments which provide (often controversial) evidence for Luttinger liquid correlations in several classes of materials. We discuss organic conductors and superconductors, inorganic charge density wave systems, and semiconductors in the quantum Hall regime.

The general approach chosen here is to give some space to the discussion of the basic methods used in this field in the last decade and to some selected examples. This necessarily requires selection, often biased by the author's prejudices, and many important papers are discussed only briefly. It is hoped that the general discussion will give tools, and that the overview on the current status give orientation to the reader to locate and appreciate the original articles relevant to him. Although I have tried to incorporate a maximum of the published literature available to me, space restrictions did not allow to do so systematically. I apologize to all those whose contributions have not received due coverage. 


\section{Chapter 2}

\section{Fermi liquid theory and its failure in one dimension}

\subsection{The Fermi liquid}

Macroscopic properties of ordinary (3D) metals can be described remarkably well by the model of a Fermi gas although the interactions are not weak. Why is this possible? The answer is provided by Landau's theory of the Fermi liquid [1, 2].

The key observation is that macroscopic properties involve only excitations of the system on energy scales (say temperatures) small compared to the Fermi energy. The state of the system can be specified in terms of its ground state, i.e. its Fermi surface, and its low-lying elementary excitations - a rarified gas of "quasi-particles". These quasi-particles evolve continuously out of the states of a free Fermi gas when interactions are switched on adiabatically, and are in one-to-one correspondence with the bare particles (adiabatic continuity). They possess the same quantum numbers as the original particles, but their dynamical properties are renormalized by the interactions. This scenario emerges because the phase space for scattering of particles is severly restricted by Fermi statistics: at low temperatures, most particles are frozen inside the Fermi sea, and only a fraction $T / T_{F} \ll 1$ participate in the scattering processes. Apart those originating from the requirement of stability there are, however, no restrictions on the magnitude of the effective interactions between the quasi-particles, as measured by the Landau parameters. The restriction to low-lying excitations implying low densities of excitations, and Fermi statistics are enough to ensure Fermi liquid properties.

The ground state of a gas of free particles is fully described by its momentum distribution function $n_{0}(\mathbf{k})$. For the interacting system, it can be specified by the quasi-particle distribution function which is the same as that of the bare particles in the free system. Excitations are then determined by the deviations they produce in the momentum distribution with respect to the ground state, $\delta n(\mathbf{k})=n(\mathbf{k})-n_{0}(\mathbf{k})$. So long as there are few excitations, $\delta n(\mathbf{k})$ is small. The change in energy $\delta E$ associated with quasi-particle 
excitations can then be expanded in powers of $\delta n(\mathbf{k})$

$$
\delta E=\sum_{\mathbf{k}}\left[\varepsilon_{0}(\mathbf{k})-\mu\right] \delta n(\mathbf{k})+\frac{1}{2} \sum_{\mathbf{k}, \mathbf{k}^{\prime}} \delta n(\mathbf{k}) f\left(\mathbf{k}, \mathbf{k}^{\prime}\right) \delta n\left(\mathbf{k}^{\prime}\right)+\ldots,
$$

where $f\left(\mathbf{k}, \mathbf{k}^{\prime}\right)$ is the quasi-particle interaction and $\mu$ is the chemical potential. Although the single-particle term is of first order in $\delta n(\mathbf{k})$ and the interaction term of second order, they are in fact of equal importance and the second term cannot be neglected: the notion of a quasi-particle making sense only in the neighbourhood of the Fermi surface, $\varepsilon_{0}(\mathbf{k})-\mu$ is small there and of the same sign as $\delta n(\mathbf{k})$.

On a more formal level, the Green function of an electron is

$$
G(\mathbf{k}, \omega)=\frac{1}{\varepsilon_{0}(\mathbf{k})-\omega-\Sigma(\mathbf{k}, \omega)},
$$

where $\varepsilon_{0}(\mathbf{k})$ is the bare dispersion and $\Sigma(\mathbf{k}, \omega)$ is the self-energy containing all the manybody effects. The poles of the Green functions give the single-particle excitation energies, and the imaginary part of the self-energy provides the damping of these excitations. $\Sigma(\mathbf{k}, \omega)$ is, for fixed $\mathbf{k}$, a smooth function of $\omega$ and continuous in $\mathbf{k}$. This guarantees solutions to the equation

$$
\varepsilon_{0}(\mathbf{k})-\omega-\Sigma(\mathbf{k}, \omega)=0,
$$

determining the single particle excitation energies. One hopes that there is only a single solution to this equation - but this need not be so. In fact, having a single solution - the quasi-particle pole with finite residue [四]

$$
z_{\mathbf{k}}=\left(1-\frac{\partial \operatorname{Re} \Sigma(\mathbf{k}, \omega)}{\partial \omega}\right)_{\omega=\varepsilon(\mathbf{k})}^{-1} \leq 1
$$

- implies a normal Fermi liquid. We shall see below that the the breakdown of Fermi liquid theory in $1 \mathrm{D}$ is signalled by the appearance of multiple solutions or vanishing of $z_{\mathbf{k}}$. The quasi-particle residue $z_{k_{F}}$ gives the magnitude of the jump of the momentum distribution function of the bare particles at the Fermi surface [四]. Expanding the self-energy to second order, the Green function close to the Fermi surface becomes

$$
G(\mathbf{k}, \omega)=G_{i n c}(\mathbf{k}, \omega)+\frac{z_{\mathbf{k}}}{\omega-v\left(|\mathbf{k}|-k_{F}\right)+i u \operatorname{sign}\left(|\mathbf{k}|-k_{F}\right)\left(|\mathbf{k}|-k_{F}\right)^{2}} .
$$

There is no damping of the quasi-particles at the Fermi surface. They will exist off the Fermi surface only to the extent that their damping is sufficiently small (their lifetime long enough) to make them behave like an eigenstate over a reasonably long time scale. Damping of a quasi-particle with energy $\omega$ is provided by complex configurations of quasiparticle-quasi-hole excitations. They also produce incoherent background $\operatorname{Im} G_{i n c}(\omega)$ in the spectral function which, interfering with the coherent part, gives $\operatorname{Im} G(\omega) \propto \omega^{2}$ for $\omega \rightarrow 0$ at finite $k$. Eq. (2.5) is to be compared to the 1D Green functions derived in Chapter 3.3, and to that of the marginal Fermi liquid whose quasi-particle residue vanishes as 13

$$
z_{\mathbf{k}} \sim-1 / \ln |\varepsilon(\mathbf{k})| \quad \text { for } \quad \varepsilon(\mathbf{k}) \rightarrow \mu=0
$$


The quasi-particle is the central concept in the theory of the Fermi liquid. From the quasi-particle picture, Landau derived, in his first paper, a Boltzmann-like transport equation for the Fermi liquid [1]. To this end, one assumes that spatially inhomogeneous excitations in the system take place on a macroscopic scale only, so that the wavevector $\mathbf{k}$ remains a good quantum number at least within a volume of macroscopic size. One can then define a local distribution function $\delta n(\mathbf{k}, \mathbf{r})$. The time evolution of this distribution is then given by

$$
\frac{\partial \delta n(\mathbf{k}, \mathbf{r})}{\partial t}+\mathbf{v}_{\mathbf{k}} \cdot \nabla \delta n(\mathbf{k}, \mathbf{r})+\delta\left(\varepsilon_{\mathbf{k}}-\mu\right) \sum_{\mathbf{k}^{\prime}} f\left(\mathbf{k}, \mathbf{k}^{\prime}\right) \mathbf{v}_{\mathbf{k}} \cdot \nabla \delta n\left(\mathbf{k}^{\prime}, \mathbf{r}\right)=I[n]
$$

where $I[n]$, the collision term, is a functional of $n(\mathbf{k}, \mathbf{r})$ and the velocity $\mathbf{v}_{\mathbf{k}}=\nabla_{\mathbf{k}} \varepsilon(\mathbf{k})$. Since $\delta n$ and $\delta\left(\varepsilon_{\mathbf{k}}-\mu\right)$ appear, it is clear that this equation applies only close to the Fermi surface. Notice that the assumption of variation of $n(\mathbf{k}, \mathbf{r})$ over macroscopic length scales implies coarse graining any underlying microscopic theory over length scales at least of the order of the thermal de Broglie length $\xi \sim v_{F} / \pi T$. $\xi$ measures the length over which the quasi-particles loose their phase coherence. Moreover, due to the collision term, (2.7) contains dissipation, produced by the elimination of degrees of freedom in the coarse graining process.

Subsequently however, Landau was able to derive the same equation from the general formalism of many-body theory without making reference to the quasi-particle picture [1], and one could conceive generalizations of the Fermi liquid theory based on this equation. For the one-dimensional Fermi liquid, however, the analogon of the Landau-Boltzmann transport equation has not yet been derived, and the usual derivation fails in 1D. In the remainder of this article, we shall base our notion of a Fermi liquid on the quasi-particle picture.

\subsection{Breakdown of Fermi liquid theory in one dimen- sion}

Adiabatic continuity is, a priori, a hypothesis which needs verification: while it works for repulsive interactions in $3 \mathrm{D}$, it cannot be justified for attractive interactions where a transition to superconductivity takes place - but neither can it be justified for repulsive interactions in $1 \mathrm{D}$, the case of highest interest in the present article. Here, we discuss where Fermi liquid theory breaks down in $1 \mathrm{D}$. The first discussion is rather qualitative and handwaving. A second one computes the perturbation corrections in the Green function of a 1D Fermi gas due to some interactions and therefore probes quasi-particles. The third part finally indicates where the derivation of Landau's quasi-particle interactions and transport equation breaks down and suggests that also the latter will have a new shape in $1 \mathrm{D}$.

On the microscopic level, the central problem in the theory of 1D interacting electrons is the Peierls instability [5], Figure 2.1: 1D electrons spontaneously open a gap at the Fermi surface when they are coupled adiabatically to phonons with wave vector $2 k_{F}$. The 
mechanism operates, however, also for electron-electron interactions. The particle-hole susceptibility in Figure 2.1 diverges as $\ln \left[\max \left(v_{F} q, \omega\right)\right]$ if momentum $2 k_{F}+q$ and frequency $\omega$ are transferred through the bubble. Its origin is the nesting property of the 1D Fermi surface: one piece of the Fermi surface can be matched identically onto the other by a "translation" with $Q= \pm 2 k_{F}$. (In higher dimensions, in the generic case, a given $2 \mathbf{k}_{F}$ only matches two points - a Fermi surface part of measure zero.) Summing up a particle-hole ladder, i.e. doing a mean-field theory, one would predict a (charge or spin) density wave instability at some finite temperature for repulsive interactions - implying that there can be no Fermi liquid in $1 D$. The finite transition temperature is, of course, unphysical and an artefact of mean-field theory. It is removed by realizing that, since the Peierls channel is as divergent as the Cooper pairing channel, both types of instabilities interfere and one has to solve at least a "parquet" of diagrams [30. The Peierls-Cooper interference conveys a marked non-mean-field character to this problem: mean-field theories are constructed by selecting one important series of diagrams. Here two of them interfere and compete! The 1D Fermi gas is inherently unstable towards any finite interaction, suggesting that it is not a good point of departure for analyzing interacting electrons in 1D. (Notwithstanding this statement, much progress in our understanding of 1D fermions is due perturbing the 1D Fermi gas by electron-electron interaction [18].) There is thus urgent need for new low-energy phenomenology, similar in spirit to the Fermi liquid picture, but adapted to the specific problems of $1 \mathrm{D}$ electrons.

The breakdown of Fermi liquid theory in 1D is also visible in a second order perturbation calculation, as we will demonstrate now. We consider a simplified problem of 1D electrons with a density-density interaction parameterized by a coupling constant $g$. We calculate the self-energy $\Sigma_{r s}(q, \omega)$ in Eq. (2.2) in second order perturbation theory. The relevant diagrams are shown in Figure 2.2. Anticipating on the next Chapters, we limit ourselves to (forward) scattering processes transferring only small momentum $q \ll k_{F}$, and discuss the relevant processes separately in order to avoid obscuring interferences. All arguments are robust, however.

We start with the process where all scattering partners are on the same side $r= \pm$ of the Fermi surface, to be called $g_{4}$ hereafter [the Hamiltonian is written out in Eq. (3.4)]. So long as $g_{4}$ is independent of momentum transfer, Hartree and Fock terms will cancel each other for scattering partners having the same spin. If they have opposite spin $\left(g_{4 \perp}\right)$, (b) and (d) are absent, and (a) only renormalizes the chemical potential. The self-energy (c) can be calculated and injected into (2.2). The pole of the Green function should give the energy for quasi-particle excitations, but here we obtain two solutions

$$
\omega=\left(v_{F} \pm\left|\frac{g_{4 \perp}}{\sqrt{8} \pi}\right|\right)\left(r k-k_{F}\right) \quad \text { !!! }
$$

This violates the single-pole assumption at the origin of the Fermi liquid. Anticipating Chapter 3 the meaning of the two poles is clear: charge-spin separation. The two poles are not converged into a single pole by higher order terms, which generate more and more poles around the two found in Eq. (2.8) and finally merge into a branch cut, giving this model the specific spectral features discussed in detail in Section 3.4. 
We now turn to forward scattering where both partners are on opposite sides of the Fermi surface [labelled $g_{2}$ hereafter, cf. Eq. (3.3)]. We drop spin indices since one has only the Hartree diagrams (a) (renormalizing again the chemical potential) and (c) both for $g_{2 \|}$ and $g_{2 \perp}$. Diagram (c) contains a counterpropagating electron-hole pair at $\pm k_{F}$. This is precisely the Peierls bubble from Fig. 2.1 which gives a logarithmic dependence to $\Sigma(k, \omega)$. The pole in the Green function (2.2) now has a residue $z_{k} \sim-1 / \ln \left|r k-k_{F}\right| \rightarrow 0$ as $k \rightarrow k_{F}$. Any quasi-particle character of the excitation fades away as we approach the Fermi surface! Again, higher order terms cannot restore the quasi-particle pole. They produce higher powers of the logarithm which sum up to a power law. These ubiquitous power laws have been mentioned in the Introduction and will be discussed in more detail in Chapter 3 .

A complete and rigorous microscopic justification of the Landau theory can be given [2]. Here, we limit ourselves to a sketch of where these arguments break down in 1D. The quasi-particle interaction $f\left(k, k^{\prime}\right)$ defined via Landau's expansion of the total energy (2.1) is related through

$$
f\left(k, k^{\prime}\right)=2 \pi i z_{k} z_{k^{\prime}} \lim _{\omega \rightarrow 0} \lim _{q \rightarrow 0} \Gamma\left(k, E_{F}, k^{\prime}, E_{F} ; q, \omega\right)
$$

to the complete particle-hole interaction vertex $\Gamma\left(k, E, k^{\prime}, E^{\prime} ; q, \omega\right)$. Notice that no momentum transfer is involved in the quasi-particle interaction. The complete particle-hole interaction $\Gamma$ is related to the irreducible one $I$ by the Bethe-Salpeter equation which we only display graphically in Figure 2.3. Singularities in $\Gamma$ are required for eventually destabilizing quasi-particles ( $\Gamma$ determines the two-particle Green function which is coupled, via the interaction, into the single-particle Green function). So long as $I$ is nonsingular, singular $\Gamma$ can only arise from the internal Green functions in the right diagram in Figure 2.3. Physically, they represent that part of the effective interaction which is mediated by propagating particles. There are, in fact, such singularities when the difference of (four-)momenta on the internal lines tends to zero. Due to the particular limit involved in Eq. (2.9), the quasi-particle interaction is not sensitive to these singularities and remains regular. The singularities matter, however, in opposite (forward scattering) limit $\omega=0, q \rightarrow 0$ when momentum transfer is allowed. Then collective (zero sound) modes can be excited. Their velocity, however, exceeds the Fermi velocity so that they do not interfere with the quasi-particles.

Now consider one dimension. As we have seen in Figure 2.1 above there is a logarithmic singularity in the (Peierls) particle-hole susceptibility at $q=2 k_{F}$. It is clear from Figure 2.3 that the Peierls bubble gives an additional divergence in the Bethe-Salpeter equation when the momentum of the internal Green functions differs by $2 k_{F}$ whereas the derivation of Landau theory assumes this vertex to be finite at $2 k_{F}$. Moreover, the Peierls divergence is worse than that at $q=0$ in that the internal momentum integrals sample the full bandwidth; at small $q$, the pole structure of the singular part is such that one only integrates over a slice of width $q$. This is why the singularity does not enter the quasiparticle interaction. The two-particle Green function then carries the singularity in $\Gamma$ into the single-particle Green function where it will ruin the quasi-particle pole. 
The quasi-particle interaction $f\left(k, k^{\prime}\right)$ in (2.9) does not involve momentum transfer between the quasi-particles. In other words, the components of the interaction which do transfer momentum are irrelevant in $3 \mathrm{D}$. This is very different from $1 \mathrm{D}$ where, on dimensional grounds, these interactions are marginal and cannot be neglected compared to those which do not transfer momentum. This finally generates charge-spin separation. We shall give a more detailed argument in the next chapter, at the end of Section 3.1.3, after we have introduced the relevant Hamiltonian. A reduction of the interactions to an effective quasi-particle interaction (2.9) cannot be operated in 1D.

Any search for an extension or a replacement of Fermi liquid theory in 1D must necessarily incorporate in a consistent manner the Peierls divergence and momentum transfer in the interaction process. This is what the Luttinger liquid approach, to be discussed in the next chapter, does. A valid though less satisfactory alternative, starting from a 1D Fermi gas, is offered by either solving parquet equations or performing renormalization group as "devices" to sum up consistently the offending divergences discussed here [18, 30]. 


\section{Chapter 3}

\section{The Luttinger model}

\subsection{Low-energy phenomenology in 1D - the Luttinger model}

\subsubsection{Ground state and elementary excitations of 1D fermions}

We have seen in the previous chapters that both the Peierls singularity and charge-spin separation, both related to the small phase space in 1D, spoil a Fermi liquid description of 1D correlated fermions and require new approaches. On the other hand, the rewarding feature of 1D physics is that the particle-hole excitations acquire well-defined particlelike dispersion in the long-wavelength limit $q \rightarrow 0$, Figure 3.1. These collective density fluctuations obey approximately bosonic commutation relations and can indeed be used to construct the new low-energy phenomenology called for.

To describe the low-energy physics, we need to know the ground state and the elementary excitations. Consider a system with $N_{0}$ electrons in a system of length $L$. In the absence of external fields, the ground state of the free system is the Fermi sea $|F S\rangle$ with $k_{F}=N_{0} \pi / 2 L$. In general, the ground state may be different, however. In a magnetic field, the number of up- and down-spins, is different, $k_{F \uparrow} \neq k_{F \downarrow}$, and in an electric field, the number of right- and left-moving fermions is different, $k_{F}^{(+)} \neq-k_{F}^{(-)}$, producing a net magnetization and current, respectively. Varying the chemical potential changes all four $k_{F}$. With respect to the reference state given by $k_{F}$, one can therefore introduce four numbers $N_{r, s}$ measuring the addition or removal of fermions, above or below the reference $k_{F}$, in the channel $(r, s)$, where $r$ labels the dispersion branch close to $r k_{F}$ and $s$ the spin. The total charge and spin as well as charge and spin currents with respect to the reference state are obtained by linear combination.

What are the elementary excitations? For the free system, one could add a fermion in a $k$-state with $|k|>k_{F}$ and create a quasi-particle. However, we have seen in the preceding chapter that these quasi-particles are not stable against turning on interactions.

Next consider particle-hole excitations $c_{k+q}^{\dagger} c_{k}|F S\rangle$, Fig. 3.1 (left). Firstly, notice that the electron and hole created travel at the same group velocity and therefore form an almost bound state which is certainly extremely susceptible to interactions, particularly in 1D. 
Secondly, for small $q$ where the dispersion is almost linear, there is a huge degeneracy $L|q| / 2 \pi$ of these excitations with energy $\omega(q)$. We can form "particles", corresponding to the linear dispersion branch $\omega(q) \propto|q|$ for $q \rightarrow 0$ in Fig. 3.1, by coherently superposing

particle-hole excitations with different $k$, Eq. (3.5) below. These fluctuations are also present in the Fermi liquid but there, the low-energy spectral region $\left(\omega<v_{F} k_{F}, 0<\right.$ $\left.q<2 k_{F}\right)$ is filled in. The presence of these finite-q low-energy states allows the decay of these excitations into their constituent quasi-particles and therefore is responsible for a kind of "chemical" equilibrium between quasi-particles and collective excitations. In 1D this decay into quasi-particles is not possible and makes these charge- (spin-) fluctuations stable elementary excitations of the system. They have bosonic commutation properties.

With respect to our reference state $|F S\rangle$, there are thus two types of elementary excitations: (i) the charge and spin and their corresponding current excitations which change the Fermi wavevectors $k_{F, s}^{(r)}$ and thus the number of fermions in the system [3], and (ii) the collective bosonic charge and spin-density fluctuations. There are no stable quasiparticles, and the addition of a fermion generates both types of elementary excitations, cf. Eq. (3.41) below.

These features are generic to 1D gapless quantum systems but are particularly prominent in the exactly solvable Luttinger model. In the following, we describe and solve this model before we turn, in the next chapter, to the reduction of microscopic lattice models (e.g. Hubbard model) onto effective Luttinger Hamiltonians.

\subsubsection{Tomonaga-Luttinger Hamiltonian}

The Tomonaga-Luttinger model describes 1D right- and left-moving fermions through the Hamiltonian [3], [7]-[9], [31]-[35]

$$
\begin{aligned}
H & =H_{0}+H_{2}+H_{4}, \\
H_{0} & =\sum_{r, k, s} v_{F}\left(r k-k_{F}\right): c_{r k s}^{\dagger} c_{r k s}: \\
H_{2} & =\frac{1}{L} \sum_{p, s, s^{\prime}}\left[g_{2 \|}(p) \delta_{s, s^{\prime}}+g_{2 \perp}(p) \delta_{s,-s^{\prime}}\right] \rho_{+, s}(p) \rho_{-, s^{\prime}}(-p), \\
H_{4} & =\frac{1}{2 L} \sum_{r, p, s, s^{\prime}}\left[g_{4 \|}(p) \delta_{s, s^{\prime}}+g_{4 \perp}(p) \delta_{s,-s^{\prime}}\right]: \rho_{r, s}(p) \rho_{r, s^{\prime}}(-p): .
\end{aligned}
$$

$c_{r k s}$ describes fermions with momentum $k$ and spin $s$ on the two branches $(r= \pm$ ) of the dispersion varying linearly $\left[\varepsilon_{r}(k)=v_{F}\left(r k-k_{F}\right)\right]$ about the two Fermi points $\pm k_{F}$.

$$
\rho_{r, s}(p)=\sum_{k}: c_{r, k+p, s}^{\dagger} c_{r, k, s}:=\sum_{k}\left(c_{r, k+p, s}^{\dagger} c_{r, k, s}-\delta_{q, 0}\left\langle c_{r, k, s}^{\dagger} c_{r, k, s}\right\rangle_{0}\right)
$$

is the density fluctuation operator (describing the "particles" introduced above), and : . . : denotes normal ordering, defined by the second equality. The Tomonaga and Luttinger models are distinguished by different cutoff prescriptions on the dispersion. In the Tomonaga model [8] there is a finite bandwidth cutoff $k_{0}$, i.e. the allowed $k$-space states for branch $r$ are $r k_{F}-k_{0}<k<r k_{F}+k_{0}$. This simulates the finite bandwidth of all 
real physical systems but, unfortunately, only allows an asymptotically exact solution. In the Luttinger model [7], on the contrary, the dispersion extents to infinity: $-\infty<k<\infty$ for both branches. In order to obtain physically meaningful results, all the negative energy states have to be occupied. The presence of these unphysical states is not expected to affect the low-energy physics of the model $\left(|\omega| \ll E_{F},|q| \ll k_{F}\right)$. (A thorough discussion of various cutoff procedures is given by Sólyom [18] and Apostol [36].) The normal ordering convention in Eqs. (3.2) and (3.5) is necessary to avoid reference to the infinite quantity $\sum_{k}\left\langle c_{r k s}^{\dagger} c_{r k s}\right\rangle$, the total particle number, which is ill-defined. The coupling constants $g_{2}$ and $g_{4}$ measure the strength of forward scattering (momentum transfer $|q| \ll k_{F}$ ) between particles on different or on the same branch of the dispersion, respectively, Figure 3.2. They may depend on the relative orientation of the spin of the scattering particles. The interaction terms with $p=0$ give the change in Hartree-Fock energy of the system upon addition of particles, and those with finite $p$ describe the scattering of the elementary excitations.

An exact solution of the Luttinger model is possible [3, 9, 31, 34] if a cutoff $\Lambda$ is imposed on the momentum transfer of the interactions [3, 31]. The coupling "constants" $g_{i}(p)$ therefore depend on the momentum transfer (below, we shall exhibit explicitly this momentum dependence only where necessary).

\subsubsection{Symmetries and conservation laws}

The possibility for an exact solution of the Luttinger model can be traced back to severe conservation laws. The Hamiltonian not only conserves the total charge and spin of the system

$$
\left[N_{\rho}, H\right]=0 \quad, \quad\left[N_{\sigma}, H\right]=0
$$

but is does so separately on each branch $r$

$$
\left[N_{r, \rho}, H\right]=0, \quad\left[N_{r, \sigma}, H\right]=0, \quad \text { or } \quad\left[N_{r, s}, H\right]=0
$$

Clearly, this implies conservation of the charge and spin currents

$$
\left[J_{\rho}, H\right]=0, \quad\left[J_{\sigma}, H\right]=0, \quad \text { or } \quad\left[J_{s}, H\right]=0 .
$$

Consequently, the Hamiltonian is invariant under the gauge transformations

$$
\Psi_{r s}(x) \rightarrow \exp \left(i \theta_{r}\right) \Psi_{r s}(x)
$$

for each branch separately. Expressed differently, the Luttinger model possesses, in addition to the usual gauge symmetry $\Psi_{r s}(x) \rightarrow \exp (i \theta) \Psi_{r s}(x)$, a chiral symmetry

$$
\Psi_{r s}(x) \rightarrow \exp (\operatorname{ir} \theta) \Psi_{r s}(x)
$$

The physical origin of these conservation laws is the restriction of the interaction Hamiltonian $\mathrm{H}_{2}+\mathrm{H}_{4}$ to small momentum transfer (forward) scattering: processes scattering particles across the Fermi surface are excluded from the model. 
For specific values of the interaction constants

$$
g_{2, \|}=g_{2, \perp} \quad \text { and } \quad g_{4, \|}=g_{4, \perp}
$$

the Hamiltonian is invariant under a spin-rotation

$$
\Psi_{r s}(x) \rightarrow \sum_{s^{\prime}} g_{s s^{\prime}} \Psi_{r s^{\prime}}(x)
$$

where $g_{s s^{\prime}}=(\exp [i \Omega \cdot \sigma])_{s s^{\prime}}$ is a $\mathrm{SU}(2)$-matrix. As will be seen below, correlation functions are spin-rotation invariant also when only the left equation in (3.11) is fulfilled.

The linear dispersion and the normal ordering involved in the density operators (3.5) makes the model charge-conjugation symmetric $\left[\Psi_{r s}(x) \rightarrow \Psi_{r s}^{\dagger}(x)\right]$. While a more complete model need not be charge conjugation symmetric, linearizing the dispersion amounts to a constant-density-of-states approximation - often employed also in higher-dimensional systems. Finally, when $g_{2, s, s^{\prime}}=g_{4, s, s^{\prime}}$ the Luttinger model can be considered as the small momentum transfer limit of a physical Hamiltonian involving only density-density interactions.

Conservation of total charge and spin applies to most models commonly studied. Their conservation separately on each branch is, however, a specific property of the Luttinger model and not shared by more realistic 1D models. There, it holds in a low-energy subspace if interaction terms not commuting with the charge and spin currents are irrelevant. If they are relevant, the low-energy physics is characterized by different (possibly reduced) symmetries and cannot be described by an effective Luttinger model. Two such interaction processes are depicted in Figure 3.3. $g_{1 \perp}$ describes exchange scattering across the Fermi surface and spoils spin current conservation but respects charge current conservation. $g_{3 \perp}$ is Umklapp scattering of two particles in the same direction across the Fermi surface and destroys charge current conservation while conserving the spin current. However, momentum conservation usually inactivates $g_{3 \perp}$, except for commensurate band fillings. Other interaction processes violating both charge and spin current conservation are possible, too.

Charge conjugation and spin rotation occur as separate symmetries because of the interactions. The free Hamiltonian has a higher symmetry which, however, is broken by the interaction terms. The $g_{2}$-interaction does not commute with the kinetic energy $\left[H_{2}, H_{0}\right] \neq 0$. It therefore can modify the ground state by exciting particle-hole pairs out of the Fermi sea. On the other hand, $g_{4}$ commutes $\left[H_{4}, H_{0}\right]=0$. With this term alone the Fermi sea remains the ground state. Its influence is limited to removing degeneracies in the excitations, as can a magnetic field or a hopping matrix element between chains.

The corresponding interactions are present also in higher dimensions. Still, it seems that they play no role there. The reason is that these interactions are marginal or scale invariant in $1 \mathrm{D}$, in a renormalization group sense, while they are irrelevant in $D>1$ and drop out of the problem. Marginality means that the coupling constant does not change under a change in the length (or energy) scale while relevance or irrelevance imply an increase resp. decrease of the coupling constant as the length (energy) scale is increased (decreased). The marginality of $g_{2}$ and $g_{4}$ can be seen by simple power counting. 
Taking the length scale to have canonical dimension $[L]=1$, the Hamiltonian has $[H]=$ -1 , the fermion operator has $\left[\Psi_{r}(x)\right]=-1 / 2$ and the density operator $\left[\rho_{r}(x)\right]=-1$. Consequently $\left[g_{2}\right]=\left[g_{4}\right]=0$ i.e. $g_{2}$ and $g_{4}$ do not change with scale. The dimension of $\Psi_{r}(x)$ can be changed by the presence of marginal operators of $g_{2}$-type but not by those of $g_{4}$-type. The dimension of $\rho_{r}(x)$ is not changed by marginal interactions. Notice that the coupling constants $g_{i}$ transfer momentum in the scattering process, and their marginality implies that this momentum transfer cannot be neglected on any length (energy) scale. In contrast, the momentum transfer of interactions in 3D can be neglected because the interaction is irrelevant (the explicit prefactor $L^{-3}$ in $H_{\text {int }}$ gives it a dimension -2 ).

To see the consequences of this marginality, and in particular of $g_{4}$, inject a particle into the second empty plane wave state above the Fermi surface $\left|\varphi_{1}\right\rangle=c_{k_{F}+4 \pi / L, s}^{\dagger}|F S\rangle$. Where can it be transferred by $H_{4}$ which conserves the energy? The only allowed process relaxes it into the first empty state above the Fermi surface and excites the last particle from the Fermi sea to the same empty state: $\left|\varphi_{2}\right\rangle=H_{4}\left|\varphi_{1}\right\rangle=c_{k_{F}+2 \pi / L,-s}^{\dagger} c_{k_{F},-s} c_{k_{F}+2 \pi / L, s}^{\dagger}$ $|F S\rangle$. Now, within this two-state subsystem $\left\{\left|\varphi_{1}\right\rangle,\left|\varphi_{2}\right\rangle\right\}$, the Hamiltonian reduces to the matrix

$$
H=\left(\begin{array}{ll}
4 v_{F} \pi / L & 2 g_{4 \perp} / L \\
2 g_{4 \perp} / L & 4 v_{F} \pi / L
\end{array}\right)
$$

The diagonal terms come from the kinetic energy, and the $1 / L$-factor comes in from the quantization of the $k$-vectors, and the off-diagonal interaction terms are proportional to $1 / L$ because of the explicit normalization factor in the Hamiltonian $H_{4}$. Interaction and kinetic energy both scale with $1 / L$ and are of equal importance! Carrying through the same argument in $3 \mathrm{D}$, the kinetic terms will continue to scale with $1 / L$ while the interaction terms scale with $1 / L^{3}$ and therefore can safely be neglected at finite momentum transfer [37]. The new eigenvalues are $(4 \pi / L)\left(v_{F} \pm g_{4 \perp} / 2 \pi\right)$ suggesting that the particles have split into two objects propagating at two different renormalized velocities $v_{\rho, \sigma}=$ $v_{F} \pm g_{4 \perp} / 2 \pi-$ charge-spin separation.

The argument continues to hold as one injects the particles at higher momenta $2 n \pi / L$ where $H_{4}$ couples it to $n-1$ other states of lower energy. It also carries over to the $g_{2^{-}}$ interaction. Due to the non-conservation of energy, however, an infinite number of states are coupled to the particle at any momentum. Momentum transfer scattering, therefore, can never be neglected in 1D, and a reduction to a quasi-particle interaction (2.9) cannot be justified in any circumstances. The marginality of forward scattering with finite momentum transfer in $1 \mathrm{D}$ is at the origin both of the anomalous correlation exponents and charge-spin separation which we shall discuss in more detail in the subsequent sections. It is the important difference to higher-dimensional systems.

\subsection{Boson solution of the Luttinger model}

A variety of solutions for the Luttinger model have been produced in the past. Historically, the first solution involved a boson representation of the Hamiltonian [9] and will be reviewed first. This solution was "completed" by the construction of a boson representa- 
tion for the fermion operators [32, 33] which has been made rigorous by Haldane [3] and Heidenreich et al. 31. It emphasizes the differences between Fermi liquids in one and in higher dimensions. Methods developed in Fermi liquid theory, more strongly emphasizing similarities between 1D and 3D Fermi liquids, have also been used to solve the Luttinger model [34, 35] and are reviewed in Section 3.5.

\subsubsection{Diagonalization of Hamiltonian}

The Tomonaga-Luttinger model (3.1), (although using Luttinger's version with infinite bands and a momentum transfer cutoff in the interactions throughout, we shall often attach Tomonaga's name to the model, too) describes excitations with respect to a ground state described by the Fermi wave vector $k_{F}=(\pi / 2)\left(N_{0} / L\right)$ where $N_{0}$ is the number of physical electrons in a chain of length $L$. Due to the unphysical negative energy states, $N_{0} \neq \sum_{r k s}\left\langle c_{r k s}^{\dagger} c_{r k s}\right\rangle_{0}$; the left-hand side of this equation is finite, the right-hand side is infinite. The infinitely extended dispersion introduces many more subtleties into the model which are crucial to obtain a correct solution. There are three important steps in achieving a complete solution of this model: (i) the realization that due to the infinite dispersion, the $\rho_{r, s}(p)$ obey exact boson commutation relations [9]; (ii) a representation of the free Hamiltonian (3.2) as a bilinear in these boson operators [9]; (iii) the explicit construction of a boson representation for the fermion operators $\Psi_{r s}(x)=(1 / \sqrt{L}) \sum_{k} c_{r k s} \exp (i k x)$.

"Normally" (the precise meaning of this will become apparent below) density operators commute $\left[\rho_{s}\left(p_{1}\right), \rho_{s}\left(p_{2}\right)\right]=0$ because their Fourier transforms $\rho_{s}(x)=\Psi_{s}^{\dagger}(x) \Psi_{s}(x)$ are local objects. This is no longer true for Luttinger's density operators because of the fermion doubling

$$
\Psi_{s}(x) \rightarrow \Psi_{r, s}(x) \quad ; \quad c_{k s}=\sum_{r= \pm} \Theta(r k) c_{r k s}
$$

$\Theta(x)$ is the step function. There is now a nonlocal relation between the physical fermions $\Psi_{s}(x)$ and the right- and left-moving $\Psi_{r, s}(x)$

$$
\Psi_{s}^{\dagger}(x)=\frac{1}{2 \pi i} \sum_{r} \int_{-L / 2}^{L / 2} d y K(y) \Psi_{r, s}^{\dagger}(x+y) \text { with } K(y)=\frac{\pi}{L} \cot \left(\frac{\pi y}{L}\right),
$$

and the density operators no longer commute.

The commutator of the density operators is

$$
\left[\rho_{r, s}(p), \rho_{r^{\prime}, s^{\prime}}\left(-p^{\prime}\right)\right]=\delta_{r, r^{\prime}} \delta_{s, s^{\prime}} \sum_{k}\left(c_{r, k+p, s}^{\dagger} c_{r, k+p^{\prime}, s}-c_{r, k+p-p^{\prime}, s}^{\dagger} c_{r, k, s}\right)
$$

In a finite band containing both $\pm k_{F}\left(c_{k, s}\right.$ without the subscript $r$ ), it is permissible to change the summation variable $k \rightarrow k+p^{\prime}$ in the second term which makes the commutator vanish. For the Tomonaga model (finite bands around $\pm k_{F}$ ), for $p \neq p^{\prime}$ one has an operator acting on the states near the band edges $r k_{F} \pm k_{0}$, and the approximate bosonic commutators of the Tomonaga model are obtained by neglecting these band edge terms. For $p=p^{\prime}$ one measures the difference in the number of occupied states at $k$ and $k+p$, i.e. 
$p$ - making the commutator a finite number. For infinite bands (Luttinger model), one manipulates the (ill-defined) difference of two infinite quantities, and one must introduce normal ordered operators, Eq. (3.5), into the right hand side of Eq. (3.16). The problem of the band edge terms is then rigorously absent since there is no band edge left, and for $p=p^{\prime}$ the argument for the Tomonaga model carries over:

$$
\begin{aligned}
{\left[\rho_{r, s}(p), \rho_{r^{\prime}, s^{\prime}}\left(-p^{\prime}\right)\right] } & =\delta_{r, r^{\prime}} \delta_{s, s^{\prime}} \sum_{k}: c_{r, k+p, s}^{\dagger} c_{r, k+p^{\prime}, s}-c_{r, k+p-p^{\prime}, s}^{\dagger} c_{r, k, s}: \\
& +\delta_{r, r^{\prime}} \delta_{s, s^{\prime}} \delta_{p, p^{\prime}} \sum_{k}\left[\left\langle n_{r, k+p, s}\right\rangle_{0}-\left\langle n_{r k s}\right\rangle_{0}\right] \\
& =-\delta_{r, r^{\prime}} \delta_{s, s^{\prime}} \delta_{p, p^{\prime}} \frac{r p L}{2 \pi} .
\end{aligned}
$$

One can safely change the summation variable in the first line of (3.17) because the operators are normal-ordered; the two terms add up to zero, leaving the contribution of the second line. In the Tomonaga model with finite bands for right- and left-movers, the boson algebra obtains approximately (for wave vectors far from the band edges) because one works with truncated density operators. The algebra (3.17) is known as the $U(1)$ Kac-Moody algebra in field theory, and the nonvanishing of the commutator (3.17) due to the infinite number of negative energy states (Luttinger model) or the cutoff procedure (Tomonaga model) is called an "anomaly".

Acting on the ground state of the free Hamiltonian $H_{0}$, the $\rho_{r, s}(p)$ behave either as creation or annihilation operators, depending on $\operatorname{sign}(p)$

$$
\rho_{+, s}(-p)|0\rangle=\rho_{-, s}(p)|0\rangle=0 \text { for } p>0 \text {. }
$$

To complete the algebra, it is necessary to construct a ladder operator $U_{r s}$ which changes the fermion number without affecting the bosonic excitations. This operator is necessary again because of the infinite dispersion: since there are no upper and lower limits to the number of particles, the number operator cannot be expressed in terms of raising and lowering operators. Haldane and Heidenreich et al. have given such a construction in terms of the bosons $\rho_{r, s}(p)$ and the fermions $\Psi_{r s}(x)$ [3, 31, cf. below.

There are several ways to see that the free fermion Hamiltonian $H_{0}$, Eq. (3.2), is equivalent to an operator bilinear in the bosons $\rho_{r s}(p)$. The simplest one 9] is to examine the commutator

$$
\left[H_{0}, \rho_{r, s}(p)\right]=v_{F} r p \rho_{r, s}(p)
$$

which is obviously compatible with

$$
H_{0}=\frac{\pi v_{F}}{L} \sum_{r, p \neq 0, s}: \rho_{r, s}(p) \rho_{r, s}(-p):+ \text { const. }
$$

The equivalence of the Hamiltonians (3.2) and (3.20) is known as Kronig's identity [38], and is valid at fixed particle number. If particles are added to the system , the "+const." becomes important, however, because one must add their kinetic energy to the Hamiltonian. We put them into the lowest available states above the Fermi sea (other states can 
be reached by acting with the boson operators). The complete Hamiltonian then takes the form

$$
\begin{aligned}
H_{0} & =\frac{\pi v_{s}}{L} \sum_{r, p \neq 0, s}: \rho_{r, s}(p) \rho_{r, s}(-p): \\
& +\frac{\pi}{2 L} \sum_{s}\left[v_{N}\left(N_{+, s}+N_{-, s}\right)^{2}+v_{J}\left(N_{+, s}-N_{-, s}\right)^{2}\right], \\
(-1)_{s}^{J} & =-(-1)_{s}^{N}, \quad\left(v_{s}=v_{N}=v_{J}=v_{F}\right),
\end{aligned}
$$

where the $N_{r, s} \equiv \rho_{r, s}(p=0)$, Eq. (3.5), are taken relative to their (infinite) ground state value and therefore measure excitations with respect to a given ground state charge. The symmetric combination $N_{s}=\sum_{r} N_{r, s}$ measures charge and the antisymmetric combination $J_{s}=\sum_{r} r N_{r, s}$ measures current excitations, both carrying spin $s$. Total charge and spin, as well as charge and spin currents, are obtained by the appropriate sums over $s$. These quantities specify the number and the left-right asymmetry of the fermions added to the reference state $\left(N_{s}=N_{0} / 2, J_{s}=0\right)$. The equality of the three velocities in (3.21) to the bare one only holds for the free model and is violated by interactions (the HartreeFock energy of the added particles appears as the $q=0$ components of the interaction Hamiltonian). Including the charge and current excitations, (3.2) and (3.21) possess the same spectrum, by construction. That the multiplicities of the levels also are equal can be proved by calculating the grand partition function both in the fermion (3.2) and in the boson (3.21) representation. Thus the fermionic and bosonic Hilbert spaces are identical.

Why are such two different representations of the same Hamiltonian possible? (i) Reconsider the elementary particle-hole excitations in Figure 3.1. They acquire a welldefined particle-like character in $1 \mathrm{D}$ as $q \rightarrow 0$. In the Luttinger model the low- $q$ branch of their dispersion is strictly linear in $q$. Decay of these excitations in the constituent particles and holes is forbidden on account of $1 \mathrm{D}$ kinematics - it would involve states in the void low-frequency part of the spectrum. There should thus be a representation of the Hamiltonian, which describes excitations, in terms of these particles alternative to the original fermionic one. Moreover, the absence of dispersion implies that these excitations do not interact: one excitation with momentum $q+q^{\prime}$ has the same energy as two excitations with momenta $q$ and $q^{\prime}$. Certainly, these collective modes also exist in higher dimensions, but so does the electron-hole continuum which permits their decay into quasi-particles and quasi-holes. (ii) An intimately related observation is that the particle and the hole created in such an excitation, travel at the same group velocity and therefore form an almost bound state which surely is extremely susceptible to dramatic modification by interactions where, in any case, momentum transfer cannot be neglected. (iii) All states with even (odd) fermion charge $N-N_{0}$ have excitation energies that are even (odd) multiples of $\pi v_{F} / L$. In other words, the spectrum effectively becomes that of a harmonic oscillator. This fact again suggests that an equivalent boson representation of $H_{0}$ should be possible. (iv) The Kac-Moody algebra (3.17) can be obtained either by representing $\rho_{r s}(p)$ as a fermion bilinear (3.5) or as the gradient of true bosonic field $\Phi_{r, s}(x)$ [Eq. (3.40) below]. Since the algebra is unique, the two representations must be equivalent. While for the noninteracting problem, the two representations are true alternatives, the 
success of bosonization is related to the fact that the bosonic one becomes more "natural" once interactions are introduced.

Now the Luttinger Hamiltonian can be diagonalized by a Bogoliubov transformation [3, 9, 31]. First transform to charge and spin variables

$$
\begin{array}{ll}
\rho_{r}(p)=\frac{1}{\sqrt{2}}\left[\rho_{r, \uparrow}(p)+\rho_{r, \downarrow}(p)\right] \quad, \quad N_{r, \rho}=\frac{1}{\sqrt{2}}\left[N_{r, \uparrow}+N_{r, \downarrow}\right], \\
\sigma_{r}(p)=\frac{1}{\sqrt{2}}\left[\rho_{r, \uparrow}(p)-\rho_{r, \downarrow}(p)\right] \quad, \quad N_{r, \sigma}=\frac{1}{\sqrt{2}}\left[N_{r, \uparrow}-N_{r, \downarrow}\right] .
\end{array}
$$

We only include the $z$-component of the spin density operator working within abelian bosonization. At this point, the $\mathrm{SU}(2)$-spin transformation properties of the fermions (3.12) has been broken down to $\mathrm{U}(1$ ) [just like the gauge transformation (3.9) for the charges], and likewise for the symmetry of the Hamiltonian, even if (3.11) is satisfied. One can keep the spin densities transforming explicitly according to $\mathrm{SU}(2)$

$$
\mathbf{S}_{r}(x)=\sum_{s, s^{\prime}} \frac{1}{2} \Psi_{r s}^{\dagger}(x) \sigma_{s s^{\prime}} \Psi_{r s^{\prime}}(x)
$$

and represent the Hamiltonian in terms of the $\mathrm{U}(1)-\rho_{r^{-}}$and $\mathrm{SU}(2)-\mathbf{S}_{r}$-fields. In this way, one can keep SU(2)-invariance manifest at every stage of the calculation. The price to be paid is, however, a significantly more complicated boson respresentation which will not be reviewed here [20, 39].

The interactions transform as

$$
g_{i \rho}=\frac{1}{2}\left(g_{i \|}+g_{i \perp}\right) \quad, \quad g_{i \sigma}=\frac{1}{2}\left(g_{i \|}-g_{i \perp}\right) .
$$

The Hamiltonian then becomes $(\nu=\rho, \sigma$ henceforth)

$$
\begin{aligned}
H_{0} & =\frac{\pi v_{F}}{L} \sum_{\nu r p \neq 0}: \nu_{r}(p) \nu_{r}(-p): \\
& +\frac{\pi}{2 L}\left[v_{N \nu}\left(N_{+\nu}+N_{-\nu}\right)^{2}+v_{J \nu}\left(N_{+\nu}-N_{-\nu}\right)^{2}\right] \quad\left(v_{N \nu}=v_{J \nu}=v_{F}\right) \\
H_{2} & =\frac{2}{L} \sum_{\nu p} g_{2 \nu}(p) \nu_{+}(p) \nu_{-}(-p) \\
H_{4} & =\frac{1}{L} \sum_{\nu r p} g_{4 \nu}(p): \nu_{r}(p) \nu_{r}(-p):
\end{aligned}
$$

We diagonalize by the canonical transformation

$$
\begin{aligned}
\tilde{H} & =e^{i S_{\nu}} H e^{-i S_{\nu}}, \quad \tilde{\nu}_{r}(p)=e^{i S_{\nu}} \nu_{r}(p) e^{-i S_{\nu}} \\
S_{\nu} & =\frac{2 \pi i}{L} \sum_{p>0} \frac{\xi_{\nu}(p)}{p}\left[\nu_{+}(p) \nu_{-}(-p)-\nu_{-}(p) \nu_{+}(-p)\right] .
\end{aligned}
$$

The $\nu_{r}$ 's explicitly transform as

$$
\tilde{\nu}_{r}(p)=v_{r}(p) \cosh \left[\xi_{\nu}(p)\right]+\nu_{-r}(p) \sinh \left[\xi_{\nu}(p)\right]
$$


and $\tilde{H}$ is diagonal under the condition

$$
K_{\nu}(p) \equiv e^{2 \xi_{\nu}(p)}=\sqrt{\frac{\pi v_{F}+g_{4 \nu}(p)-g_{2 \nu}(p)}{\pi v_{F}+g_{4 \nu}(p)+g_{2 \nu}(p)}} .
$$

For repulsive interactions, $K_{\nu}<1$ while for attraction $K_{\nu}>1$. The diagonal form is then

$$
\begin{aligned}
& \tilde{H}=\frac{\pi}{L} \sum_{r \nu p \neq 0} v_{\nu}(p): \tilde{\nu}_{r}(p) \tilde{\nu}_{r}(-p): \\
&+\quad \frac{\pi}{2 L}\left[v_{N \nu}\left(N_{+\nu}+N_{-\nu}\right)^{2}+v_{J \nu}\left(N_{+\nu}-N_{-\nu}\right)^{2}\right] \\
& \text { with } \quad v_{N \nu} v_{J \nu}=v_{\nu}^{2} \text { i.e. } v_{N \nu}=v_{\nu} / K_{\nu} \quad \text { and } \quad v_{J \nu}=v_{\nu} K_{\nu}
\end{aligned}
$$

(The $N_{r \nu}$-operators are not changed by the canonical transformation.) The renormalized charge and spin fluctuation velocity is

$$
v_{\nu}(p)=\sqrt{\left[v_{F}+\frac{g_{4 \nu}(p)}{\pi}\right]^{2}-\left[\frac{g_{2 \nu}(p)}{\pi}\right]^{2}}
$$

and therefore

$$
v_{N \nu}=v_{F}+g_{4 \nu}+g_{2 \nu} \quad, \quad v_{J \nu}=v_{F}+g_{4 \nu}-g_{2 \nu},
$$

and the limit $p \rightarrow 0$ is implied whenever $p$ is not exhibited explicitly. Due to the momentum transfer cutoff $\Lambda$, we have asymptotically

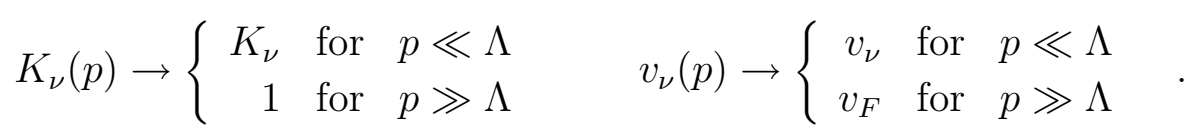

Eqs. (3.31) and (3.32) are the central constitutive relations for the Luttinger model and the Luttinger liquid hypothesis discussed in the next chapter will postulate that these relations continue to hold in a low-energy subspace of all solvable gapless 1D models. The quantity $K_{\nu}(p \rightarrow 0)$, Eq. (3.30), is the essential renormalized coupling constant for each degree of freedom, and physically plays the role of a stiffness constant. $K_{\nu}$ governs the power-law decay of most correlation functions. The two parameters $v_{\nu}$ and $K_{\nu}$ completely describe the low-energy physics of each degree of freedom $\nu$ of the model. That there are just two such parameters is not surprising: the Hamiltonian has only two parameters $g_{2 \nu}$ and $g_{4 \nu}$, and we just get back what we have put in. Important are the following facts: (i) the three different velocities in the problem are all renormalized by the interactions, Eq. (3.32), and describe different physical processes. $v_{\nu}$, the renormalized Fermi (or "sound") velocity governs the bosonic excitations; $v_{N \nu}$ is related to the fermionic charge excitations, i.e., for $\nu=\rho$ measures the shift in chemical potential upon varying the Fermi wave vector $\delta \mu=v_{N \rho} \delta k_{F}$ and, for $\nu=\sigma$ the relation of the magnetic field to the magnetization $M=v_{N \sigma}\left(k_{F \uparrow}-k_{F \downarrow}\right) . \quad v_{J \nu}$ finally measures the energy necessary to create persistent charge or spin currents on the periodic chain. (ii) All three velocities are properties of the spectrum of the model. Spectra can, however, be calculated either exactly by Bethe Ansatz (e.g. Hubbard model) or to high accuracy with numerical methods, and 
the velocities can then be determined. (iii) The three velocities determine the renormalized coupling constant $K_{\nu}$ which in turn determines all correlation functions of the Luttinger model. It is now obvious how Eq. (3.32) turns the Luttinger model into a very useful device for accessing the correlation functions of all 1D gapless models.

One prominent property of the Luttinger model - charge-spin separation - is manifest here: charge and spin fluctuations propagate with different velocities and will therefore separate in time. In realistic models, charge-spin separation will be dynamically generated close to their Fermi surface. In the Luttinger model describing just this subspace, it has become a manifest property of the model.

Collective charge density and spin density fluctuations propagating with different velocities do also occur in higher dimensional models, and in particular in the Fermi liquid. This is not special to 1D. Dramatic consequences in 1D arise, however, from the lack of robustness of hypothetical quasi-particles with respect to these elementary excitations separating in time. Quasi-particles do not exist in 1D systems with charge-spin separation. This may again be traced back to the lack of a continuum of low-energy excitations for $0 \leq q \leq 2 k_{F}$, (Figure 3.1): in 1D there is no way how these collective modes can decay into the hypothetical constituent quasi-particles (holes) which therefore never reappear once interactions have been introduced. The absence of quasi-particles is most directly seen in the single-particle spectral function which cannot be written in a form similar to Eq. (2.5). Detailed results can be found in Section 3.4.

Labelling the charge-localization (Mott-Hubbard) transitions generated by strong Coulomb interactions as "charge-spin separation" is somewhat misleading. It happens in higher dimensions, too. At issue are the excitations out of this state, and whether there are quasi-particles at low energies. Of course, there may be borderline cases, where the quasi-particle residue in (2.5) is small but finite, and most of the spectral weight resides in the collective modes.

Charge-spin separation is also visible in the many-particle correlation functions. This is trivial, and also happens in higher dimension, for the small- $q$ parts of density and spin density correlation functions. The novel feature of the correlation functions in 1D is the appearance of two separate singularities close to $2 k_{F}$ (and, partly, higher multiples thereof) where a single one is expected in the absence of charge-spin separation. This will also be discussed in Section 3.4. Before, we need to find a practical representation of the fermion operator in terms of the bosons diagonalizing the Hamiltonian in order to be able to calculate correlation functions.

\subsubsection{Bosonization}

A completely satisfactory boson solution of the Luttinger model also requires an explicit representation of the fermion operators $\Psi_{r s}(x)$ in terms of the bosons $\rho_{r s}(p)$. Then any correlation function can be given an equivalent boson representation and, the diagonal Hamiltonian being a simple boson bilinear, the calculation of any of these correlation functions becomes almost trivial, reducing to Gaussian averages.

Pioneering work in this direction was performed by Luther and Peschel [32] and Mattis 
[33]. They proposed a bosonization formula which allowed an asymptotic calculation of correlation functions but was certainly not an operator identity transforming between fermions and bosons. The cutoff procedures and the interpretation of these cutoffs were ambiguous [36]. Field theorists proposed similar constructions at the same time [40].

A precise formulation of such an operator identity was given independently by Haldane [3] and Heidenreich et al. [31], and involves the construction of the (unitary) ladder operator $U_{r, s}$. This operator increases by unity the number of fermions with spin $s$ on branch $r$ and must commute with the boson operators with finite momentum. It is sufficient for that purpose to consider states $\left|N_{r, s}\right\rangle$ where all states below a certain wave vector are filled and above empty:

$$
U_{r, s}\left|N_{r, s} N_{\bar{r}, \bar{s}}\right\rangle=\left|N_{r, s}+1 N_{\bar{r}, \bar{s}}\right\rangle \quad, \quad\left[\rho_{r, s}(p \neq 0), U_{r^{\prime}, s^{\prime}}\right]=0
$$

in evident notation. A natural guess is to put the new fermion into the first free level above the reference state, occupied up to $\left|k_{F}+2 \pi N_{r, s} / L\right|$,

$$
\begin{aligned}
U_{r, s} & =\frac{1}{\sqrt{L}} \sum_{k} c_{r k s}^{\dagger} \delta\left(r k-\left[k_{F}+\frac{\left(2 N_{r, s}+1\right) \pi}{L}\right]\right) \\
& =\frac{1}{\sqrt{L}} \int_{0}^{L} d x \Psi_{r, s}^{\dagger}(x) \exp \left(i r\left[k_{F}+\frac{\left(2 N_{r, s}+1\right) \pi}{L}\right] x\right) .
\end{aligned}
$$

Its commutator with the bosons does, however, not vanish

$$
\left[\rho_{r, s}(p), U_{r^{\prime}, s^{\prime}}\right]=\frac{\delta_{r, r^{\prime}} \delta_{s, s^{\prime}}}{\sqrt{L}} \int_{0}^{L} d x e^{i p x} \Psi_{r, s}(x) \exp \left(i r\left[k_{F}+\frac{\left(2 N_{r, s}+1\right) \pi}{L}\right] x\right) .
$$

The idea now is to introduce, into Eq. (3.37), a bosonic field $\phi_{r, s}(x)$ whose commutators with $\rho_{r, s}(p)$ compensate the unwanted commutator from $\Psi_{r, s}$. One then has

$$
\begin{gathered}
U_{r, s}=\frac{1}{\sqrt{L}} \int_{0}^{L} d x e^{i r k_{F} x} e^{-i \phi_{r, s}^{\dagger}(x)} \Psi_{r, s}^{\dagger}(x) e^{-i \phi_{r, s}(x)} \\
\text { with } \\
\phi_{r, s}(x)=-\frac{\pi r x}{L} N_{r, s}+\lim _{\alpha \rightarrow 0}\left(\frac{2 \pi i}{L} \sum_{p \neq 0} \frac{e^{-\alpha|p| / 2-i p x}}{|p|} \Theta(r p) \rho_{r, s}(-p)\right)
\end{gathered}
$$

which is the desired operator. This expression can be inverted for $\Psi_{r, s}(x)$, now given in terms of bosons and the ladder operator, and compactified into

$$
\Psi_{r s}(x)=\lim _{\alpha \rightarrow 0} \frac{e^{i r\left(k_{F}-\pi / L\right) x}}{\sqrt{2 \pi \alpha}} U_{r s}^{\dagger} \exp \left(\frac{-i}{\sqrt{2}}\left[r \Phi_{\rho}(x)-\Theta_{\rho}(x)+s\left\{r \Phi_{\sigma}(x)-\Theta_{\sigma}(x)\right\}\right]\right) .
$$

The two phase fields are

$$
\Phi_{\nu}(x)=-\frac{i \pi}{L} \sum_{p \neq 0} \frac{e^{-\alpha|p| / 2-i p x}}{p}\left[\nu_{+}(p)+\nu_{-}(p)\right]-\left(N_{+, \nu}+N_{-\nu}\right) \frac{\pi x}{L},
$$

and

$$
\Theta_{\nu}(x)=\frac{i \pi}{L} \sum_{p \neq 0} \frac{e^{-\alpha|p| / 2-i p x}}{p}\left[\nu_{+}(p)-\nu_{-}(p)\right]+\left(N_{+, \nu}-N_{-\nu}\right) \frac{\pi x}{L}
$$


and are constructed from the $\phi_{r, s}$ and $\phi_{r, s}^{\dagger}$ plus commutator terms. The charge density operator is related to $\Phi_{\rho}$ by

$$
\rho(x)=\sqrt{2}\left[\rho_{+}(x)+\rho_{-}(x)\right]=-\frac{\sqrt{2}}{\pi} \frac{\partial \Phi_{\rho}(x)}{\partial x},
$$

where the factor $\sqrt{2}$ comes from (3.22), and there is an analogous expression for the spin density. $\Theta_{\nu}(x)$ is related to the momentum canonically conjugate to $\Phi_{\nu}(x)$

$$
\Pi_{\nu}(x)=\frac{1}{L} \sum_{p \neq 0} e^{-\alpha|p| / 2-i p x}\left[\nu_{+}(p)-\nu_{-}(p)\right]+\left(N_{+, \nu}-N_{-, \nu}\right) \frac{\pi}{L}
$$

by

$$
\Theta_{\nu}(x)=\pi \int_{-\infty}^{x} d z \Pi_{\nu}(z)
$$

and the commutation relations are

$$
\begin{gathered}
{\left[\Phi_{\nu}(x), \Pi_{\nu^{\prime}}\left(x^{\prime}\right)\right]=i \delta_{\nu, \nu^{\prime}} \delta\left(x-x^{\prime}\right)} \\
{\left[\Phi_{\nu}(x), \Theta_{\nu^{\prime}}\left(x^{\prime}\right)\right]=i \frac{\pi}{2} \delta_{\nu, \nu^{\prime}} \operatorname{sign}\left(x^{\prime}-x\right) .}
\end{gathered}
$$

We can rewrite the Hamiltonian in terms of these phase fields as

$$
H=\frac{1}{2 \pi} \sum_{\nu} \int d x\left\{v_{J \nu} \pi^{2} \Pi_{\nu}^{2}(x)+v_{N \nu}\left(\frac{\partial \Phi_{\nu}(x)}{\partial x}\right)^{2}\right\},
$$

making obvious the equivalence to the Gaussian model of statistical mechanics. Under the Bogoliubov transformation (3.28), the phase fields transform as

$$
\Phi_{\nu} \rightarrow \Phi_{\nu} \sqrt{K_{\nu}} \quad \text { and } \quad \Theta_{\nu} \rightarrow \Theta_{\nu} / \sqrt{K_{\nu}}
$$

if we neglect the momentum dependence of the interactions $g(p)$ so that the $K_{\nu}$ can be taken outside the summations in (3.42) and (3.43). These expressions can now be employed for calculating arbitrary correlation functions. Examples are given in the following.

There is also a more physical way of arriving at the general boson structure of the fermion operators [41, 42]. Define a boson field $\Phi_{r, s}(x)$ by $\partial \Phi_{r, s}(x) / \partial x=-\pi \rho_{r, s}(x)$ where $\rho$ describes density fluctuations. Introducing a particle at site $x$ creates a kink of amplitude $\pi$ in the field $\Phi_{r, s}$, i.e. the phases of all other particles have to shift to accommodate the new particle. This field can be considered as a dynamical implementation of the Fermi surface phase shifts appearing in Anderson's arguments in favour of a Luttinger liquid in 2D [11]. Since displacement operators are exponentials of momentum operators, one could guess $\Psi_{r, s}(x) \sim \exp \left[i \pi \int_{-\infty}^{x} d z \Pi_{r, s}(z)\right]$ where $\Pi_{r, s}(z)$ is the momentum canonically conjugate to $\Phi_{r, s}(x)$. This operator commutes, however, with itself. The required change of sign at $x=x^{\prime}$ is achieved by multiplying with $\exp \left[ \pm i \Phi_{r, s}(x)\right]$ which yields

$$
\Psi_{r, s}(x) \approx \lim _{\alpha \rightarrow 0} \frac{1}{2 \pi \alpha} \exp \left[i r k_{F} x-i r \Phi_{r, s}(x)+i \pi \int_{-\infty}^{x} d z \Pi_{r, s}(z)\right] .
$$

This is essentially the Luther-Peschel-Mattis formula [32, 33] which contains all the important bosonic terms for the calculation of physical properties but does not have the status of an operator identity in the full Hilbert space of the Luttinger model. 


\subsubsection{Spinless fermions}

At various stages of this article, we will need spinless fermions, either because of their physical relevance as elementary charge excitations (holons) in the Hubbard and related models, or just for simplification. Here, we compile the most important formulae from the preceding paragraphs for spinless fermions.

The Hamiltonian is obtained by dropping the spin label and summations in (3.2) (3.4), i.e.

$$
\begin{aligned}
H & =\frac{\pi v_{F}}{L} \sum_{r p}: \rho_{r}(p) \rho_{r}(-p):+\frac{\pi v_{F}}{2 L}\left(N^{2}+J^{2}\right) \\
& +\frac{1}{L} \sum_{p}\left(g_{2}(p) \rho_{+}(p) \rho_{-}(p)+\frac{g_{4}(p)}{2} \sum_{r}: \rho_{r}(p) \rho_{r}(-p):\right)
\end{aligned}
$$

with $N, J=N_{+} \pm N_{-}$. The Hamiltonian is diagonalized as

$$
\tilde{H}=\sum_{r} \frac{\pi v(p)}{L}: \tilde{\rho}_{r}(p) \tilde{\rho}_{r}(-p):+\frac{\pi}{2 L}\left(v_{N} N^{2}+v_{J} J^{2}\right)+\text { const. }
$$

with the velocities

$v(p)=\sqrt{\left[v_{F}+\frac{g_{4}(p)}{2 \pi}\right]^{2}-\left[\frac{g_{2}(p)}{2 \pi}\right]^{2}}, \quad v_{N}=v_{F}+\frac{g_{4}(0)+g_{2}(0)}{2 \pi}, \quad v_{J}=v_{F}+\frac{g_{4}(0)-g_{2}(0)}{2 \pi}$,

and the stiffness "constant"

$$
K(p)=\sqrt{\frac{2 \pi v_{F}+g_{4}(p)-g_{2}(p)}{2 \pi v_{F}+g_{4}(p)+g_{2}(p)}} .
$$

Finally, the bosonization identity for spinless fermions is

$$
\Psi_{r}(x)=\lim _{\alpha \rightarrow 0} \frac{e^{i r\left(k_{F}-\pi / L\right) x}}{\sqrt{2 \pi \alpha}} U_{r}^{\dagger} \exp (-i[r \Phi(x)-\Theta(x)])
$$

The fields $\Phi(x)$ and $\Theta(x)$ are given by the expressions (3.42) and (3.43) for the charges, and the operators $\rho_{r}(p)$ now refer to spinless fermions. With these fields, the Hamiltonian the has the following phase representation

$$
H=\frac{1}{2 \pi} \int d x\left\{v_{J} \pi^{2} \Pi^{2}(x)+v_{N}\left(\frac{\partial \Phi(x)}{\partial x}\right)^{2}\right\} .
$$

\subsection{Physical Properties of the Luttinger Model - Ther- modynamics and Correlation Functions}

The machinery set up in the preceding section is extremely useful in calculating correlation functions. A remarkable feature of the Luttinger model is that all correlation functions can be calculated exactly. With the boson representation of an operator, all the expectation 
values reduce to Gaussian averages, as we shall show on some examples here. The linear response of an operator $B$ in a system described by a Hamiltonian $H_{0}$, coupled to an external field $a(x, t)$ by the operators $A(x)$, i.e. $H=H_{0}+\int d x a(x, t) A(x)$ is related, through the Kubo formulae, to correlation functions of the system in the absence of the external field (in the interaction picture and assuming translational invariance)

$$
\begin{aligned}
\langle B(x, t)\rangle & =\left\langle B^{(a=0)}(x, t)\right\rangle+\int_{-\infty}^{\infty} \chi_{B A}\left(x-x^{\prime}, t-t^{\prime}\right) a\left(x^{\prime}, t^{\prime}\right) d x^{\prime} d t^{\prime}, \\
\chi_{B A}(x, t) & =-i \Theta(t)\langle[B(x, t), A(0,0)]\rangle_{a=0} .
\end{aligned}
$$

$\chi$ is called susceptibility, response function, retarded correlation function, etc. There are many closely related functions and below, we shall denote all of them as $R_{B A}$ or, if symmetric, simply as $R_{A}$, with some exception for cases of special relevance. Within linear response theory, the Luttinger model can make predictions for all possible measurements.

\subsubsection{Thermodynamics and transport}

The Luttinger model has a specific heat linear in temperature

$$
C(T)=\gamma T \quad, \quad \frac{\gamma}{\gamma_{0}}=\frac{1}{2}\left(\frac{v_{F}}{v_{\rho}}+\frac{v_{F}}{v_{\sigma}}\right) .
$$

The linearity is both characteristic of the underlying fermions (linear specific heat in any dimension) as well as of the bosonic excitations (phonons in 1D also have a linear specific heat). $\gamma_{0}$ is the coefficient of free electrons which can be calculated from both representations as

$$
\gamma_{0}=\frac{\pi^{2} k_{B}^{2}}{3} N\left(E_{F}\right)=\frac{2 \pi k_{B}^{2}}{3 v_{F}}
$$

the density of states of the free Luttinger model being a constant $N(E)=2 / \pi v_{F}$ including spin and both branches. The spin susceptibility and compressibility are

$$
\frac{1}{\chi}=\frac{1}{L} \frac{\partial^{2} E_{0}(\sigma)}{\partial \sigma^{2}} \quad, \quad \frac{1}{\kappa}=\frac{1}{L} \frac{\partial^{2} E_{0}(n)}{\partial n^{2}},
$$

where $E_{0}$ is the ground state energy as a function of the particle (spin) density $n(\sigma)$. Throughout this article, we denote the average particle density (band filling factor) by $n$ and the density fluctuations by $\rho(x)$. The susceptibilities are renormalized by the interactions

$$
\chi=\frac{2 K_{\sigma}}{\pi v_{\sigma}}=\frac{2}{\pi v_{N \sigma}} \quad \text { and } \quad \kappa=\frac{2 K_{\rho}}{\pi v_{\rho}}=\frac{2}{\pi v_{N \rho}} .
$$

They are related to the renormalized velocities for the charge (spin) excitations defined in Eq. (3.34). This is expected, of course, because $v_{N \nu}$ measures the change in energy upon changing the number of electrons in the system, cf. (3.31). As we shall see below, spin-rotation invariance requires $K_{\sigma}=1$.

The electrical conductivity is determined from the current-current correlations through the Kubo formula

$$
\sigma(\omega)=\frac{i}{\omega}\left[\frac{D}{\pi}+R_{j}^{R}(\omega)\right]
$$


where the first term is the diamagnetic part and the (second) paramagnetic term is given in terms of the retarded current-current correlation function

$$
R_{j}^{R}(\omega)=-\frac{i}{L} \int_{0}^{L} d x \int_{0}^{\infty} d t\langle[j(x, t), j(0,0)]\rangle e^{i \omega t}
$$

The Drude weight $D$ in fact is a susceptibility and is related to the derivative of the ground state energy with respect to an applied flux $\Phi$ 43.

$$
D=\left.\frac{\pi}{2 L} \frac{\partial^{2} E_{0}(\Phi)}{\partial \Phi^{2}}\right|_{\Phi=0}=2 v_{J \rho}
$$

A flux creates a "persistent" current in a Luttinger ring, and the appearance of $v_{J \rho}$ here should not surprise from (3.31) again. $2 v_{J \rho}=2 v_{\rho} K_{\rho}$ plays the role of the plasma frequency in $1 \mathrm{D} 44$.

One has to be careful in the definition of the current operators. Naively, one has $j(x)=\sqrt{2} v_{F}\left[\rho_{+}(x)-\rho_{-}(x)\right]=\sqrt{2} v_{F} \Pi_{\rho}(x)$. A more careful evaluation via the continuity equation $\partial_{t} \rho(x t)+\partial_{x} j(x t)=0$ gives, however,

$$
j(x t)=\frac{\sqrt{2}}{\pi} \frac{\partial}{\partial t} \Phi_{\rho}(x t)=\sqrt{2} v_{\rho} K_{\rho} \Pi_{\rho}(x t)=\sqrt{2} v_{J \rho} \Pi_{\rho}(x t) .
$$

The difference is due to the fact that, in the Luttinger model, $g_{2}$ may be different from $g_{4}$ and the density does not necessarily commute with the interaction Hamiltonian, as it does in a well-defined lattice model. Notice, however, that $v_{\rho} K_{\rho}=v_{J \rho}$; if the Luttinger model is derived from a well-defined lattice model with density-density interactions only, $g_{2 \nu}=g_{4 \nu}$ is required and, using Eq. (3.34), one obtains $v_{J \rho}=v_{F}$, i.e. the current operators are not renormalized by interactions. This applies to galilean invariant models in general where, in the limit $q \rightarrow 0$, the current becomes proportional to momentum which is conserved by the interactions [45]. Consequently, as can be shown by two partial integrations on Eq. (3.65) producing

$$
\sigma(\omega)=-\lim _{q \rightarrow 0} \operatorname{Re}(i \omega)^{-3} \int_{0}^{\infty} d t e^{i \omega t}\langle[[H, j(q t)],[H, j(q, 0)]]\rangle,
$$

one has $R_{J}^{R}(\omega) \equiv 0$ 445, so that the conductivity reduces to a pure Drude peak

$$
\sigma(\omega)=2 v_{J \rho} \delta(\omega)=2 v_{F} \delta(\omega)
$$

with an interaction-independent strength. This relation has been derived by a number of people 44, 46, 47, 48.

There are a few most remarkable facts about these unspectacular formulae. (i) The finiteness of the susceptibilities characterizes the system as a "normal metal". It is highly nontrivial in view of the ubiquitous divergences we shall encounter in the following sections. The physical origin lies in the strong conservation laws of the $1 \mathrm{D}$ phase space in the absence of backscattering [46]. (ii) These quantities can be calculated both in a fermion representation where one considers the charge excitations $N_{\nu}$ and uses (3.62), and from the $q \rightarrow 0$ limit of bosonic correlation functions which we shall compute in Section 3.5. 
The result is the same. The reason will be given below [47]. (iii) The boson representation gives the susceptibilities in absolute magnitude for lattice models provided parameters are identified correctly [47]. One can invert the procedure and use these relations to identify the parameters of a low-energy boson theory for lattice models from (3.62) [10, 48, 49]. (iv) They can be obtained from the energy alone which can be calculated either from an exact solution or accurately with numerical diagonalization where correlation functions are not readily available 44, 49.

\subsubsection{Single- and two-particle correlation functions}

The thermodynamic properties do not differ from the Fermi liquid. There, compressibility and susceptibility are renormalized by interactions, too, and the renormalization is given by the Landau parameters $F_{0}^{s}$ and $F_{0}^{a}$. We neither see the anomalous power-laws not the effects of charge-spin separation highlighted earlier. To this end, we carry on to the spaceand/or time-dependent correlation functions

$$
R_{O}(x, t)=-i \Theta(t)\left\langle\left[O(x, t), O^{\dagger}(0,0)\right]_{ \pm}\right\rangle
$$

of various operators $O$ of interest. $[\ldots]_{ \pm}$denotes commutator or anticommutator for bosons and fermions, respectively. Other, e.g. time-ordered, correlation functions are obtained in a similar way.

We give a rather explicit calculation for the single-electron Green function

$$
G_{r s}(x t)=-i \Theta(t)\left\langle\left\{\Psi_{r s}(x t), \Psi_{r s}^{\dagger}(00)\right\}\right\rangle \equiv-i \Theta(t)(\tilde{G}(x t)+\tilde{G}(-x-t))
$$

where $\Psi_{r, s}(x)$ has been defined in Eq. (3.41), to sketch how such a calculation works in practice, and to give some formulae useful for the work with boson operators. In (3.71), we have incorporated that $G$ is diagonal in $r$ and $s$. Using the bosonization identity (3.41) in (3.71), we first commute the $U_{r s}^{\dagger}$-operator from $\Psi_{r, s}(x)$ at the left of the expression through the exponentials until it arrives at the right, where $\Psi_{r s}^{\dagger}(0)$ has a $U_{r s}$. Being unitary, we have $U_{r s}^{\dagger} U_{r s}=1$. What are the terms we pick up during the commutations? $U_{r s}$ commutes with $\rho_{r s}(p \neq 0)$ by construction, so that the only nonvanishing terms come from the operators $N_{r s}$ measuring the charge excitations in the phase fields $\Phi_{\nu}$ and $\Theta_{\nu}$. These terms, however, involve prefactors $1 / L$ so that their contribution vanishes in the limit $L \rightarrow \infty$. If we are interested in this thermodynamic limit, we can neglect both the $U_{r s^{-}}$and $N_{r s^{-}}$operators altogether. We see that the Luther-Peschel-Mattis formula (3.51) (neglecting the $U_{r, s^{-}}$and $N_{r, s^{-}}$-operators) gives the exact asymptotic behaviour of the Green function! (This statement is not completely true for the many-particle functions: $U_{r s}$ anticommutes with $U_{r^{\prime} s^{\prime}}$ if at least one index is different. When one considers operators $O$ pairing $\Psi_{r, s}(x)$ with different indices, as we do in almost all two-particle functions below, the $U_{r s}$ will produce phase factors. The exponent of the power-law is unaffected by these phase factors but logarithmic or prefactor corrections crucially depend on them [50].)

After diagonalizing the Hamiltonian, the Green function becomes (dropping the indices 
$r$ and $s$ )

$$
\begin{aligned}
\tilde{G}(x t) & =\lim _{\alpha \rightarrow 0} \frac{e^{i r k_{F} x}}{2 \pi \alpha}\left\langle\exp \left(-\frac{i}{\sqrt{2}}\left[r \tilde{\Phi}_{\rho}(x t)-\tilde{\Theta}_{\rho}(x t)\right]\right) \exp \left(\frac{i}{\sqrt{2}}\left[r \tilde{\Phi}_{\rho}(00)-\tilde{\Theta}_{\rho}(00)\right]\right)\right\rangle_{\rho} \times \\
& \times\left\langle\exp \left(-\frac{i}{\sqrt{2}}\left[r \tilde{\Phi}_{\sigma}(x t)-\tilde{\Theta}_{\sigma}(x t)\right]\right) \exp \left(\frac{i}{\sqrt{2}}\left[r \tilde{\Phi}_{\sigma}(00)-\tilde{\Theta}_{\sigma}(00)\right]\right)\right\rangle_{\sigma} .
\end{aligned}
$$

However, we keep the momentum dependence of the interactions and do not use (3.50); we denote the transformed fields by $\tilde{\Phi}_{\nu}$ and $\tilde{\Theta}_{\nu}$. Moreover, since the $\rho$-phase fields commute with the $\sigma$-fields, we have separated the exponentials into products involving only $\rho$ and $\sigma$ separately. $\langle\ldots\rangle_{\nu}$ denotes the expectation value with the $\nu$-part of the Hamiltonian. Next we use the important relation

$$
e^{A} e^{B}=e^{A+B} e^{[A, B] / 2} \quad \text { valid if }[A, B] \in \mathbb{C}^{H}
$$

to merge all $\nu$-phase fields into one exponential. The commutators contribute $\exp \left[C_{\nu}(x t)\right]$ with

$$
C_{\nu}(x t)=-\frac{\pi}{L} \sum_{p \neq 0} \frac{e^{-\alpha|p|} e^{-i p x}}{p}\left(\left[K_{\nu}(p)+\frac{1}{K_{\nu}(p)}\right] i \sin \left[v_{\nu}(p) p t\right]+2 r \cos \left[v_{\nu}(p) t\right]\right) .
$$

The expectation value $\left\langle e^{A+B}\right\rangle \equiv \exp \left[D_{\nu}(x t)\right]$ is evaluated using

$$
\langle\exp A\rangle=\exp \left(\frac{1}{2}\left\langle A^{2}\right\rangle\right)
$$

valid for a linear form in boson operators whose exponential is averaged with a harmonic oscillator (Gaussian) Hamiltonian. We find

$$
\begin{aligned}
& D_{\nu}(x t)=\frac{\pi^{2}}{4 L^{2}} \sum_{p, p^{\prime} \neq 0} \frac{e^{-\alpha\left(|p|+\left|p^{\prime}\right|\right) / 2}}{p p^{\prime}} \times \\
& \quad \times \sum_{R= \pm}\left\langle\nu_{R}(p) \nu_{R}\left(p^{\prime}\right)\right\rangle \prod_{P=p, p^{\prime}}\left(r \sqrt{K_{\nu}(P)}+R \frac{1}{\sqrt{K_{\nu}(P)}}\right)\left(e^{-i P\left(x-R v_{\nu}(P) t\right)}-1\right)
\end{aligned}
$$

with

$$
\left\langle\nu_{r}(p) \nu_{r}\left(p^{\prime}\right)\right\rangle=\delta_{p,-p^{\prime}} \frac{L|p|}{2 \pi}\left(\frac{\Theta(r p)}{\exp \left[v_{\nu}(p) p / 2 k T\right]-1}+\Theta(-r p)\left\{1+\frac{1}{\exp \left[v_{\nu}(p) p / 2 k T\right]-1}\right\}\right) .
$$

For $T=0$, to be treated first, the Bose-Einstein distribution vanishes for $p \neq 0$, and we can rearrange $e^{C_{\nu}} e^{D_{\nu}}$ so that

$$
\begin{aligned}
C_{\nu}(x t)+D_{\nu}(x t) & =-\frac{1}{2}\left[V_{+}^{\nu}(x t)+V_{-}^{\nu}(x t)-2 r V_{0}^{\nu}(x t)\right] \\
V_{ \pm}^{\nu}(x t) & =\frac{1}{2} \int_{0}^{\infty} \frac{d p}{p} e^{-\alpha p} K_{\nu}^{ \pm 1}(p)\left[1-\cos (p x) e^{-i v_{\nu}(p) p t}\right], \\
V_{0}^{\nu}(x t) & =\frac{i}{2} \int_{0}^{\infty} \frac{d p}{p} e^{-\alpha p} \sin (p x) e^{-i v_{\nu}(p) p t}
\end{aligned}
$$


All correlation functions can be expressed in terms of $V_{ \pm}$and $V_{0}$.

Now remember that in the Luttinger model a momentum transfer cutoff must be imposed on the interactions, and the asymptotic values of $K_{\nu}$ and $v_{\nu}$ are given by (3.35). Taking e.g. $V_{+}$, we split the integral in two terms by adding and subtracting [...] on the right-hand side [51, 52]. Taking together $\left(K_{\nu}-1\right)[\ldots]$, the important contributions will come from $p \ll 1 / \Lambda$, and we can replace $v_{\nu}(p) \rightarrow v_{\nu}$ there. The contribution of this term to $V_{+}$then becomes

$$
\frac{K_{\nu}-1}{4} \ln \left(\frac{\Lambda_{+}^{\nu}+i v_{\nu} t+i x}{\Lambda_{+}^{\nu}}\right)+\frac{K_{\nu}-1}{4} \ln \left(\frac{\Lambda_{+}^{\nu}+i v_{\nu} t-i x}{\Lambda_{+}^{\nu}}\right)
$$

where the cutoff $\Lambda_{+}^{\nu}$ is given by

$$
\ln \left(\frac{\Lambda_{+}^{\nu}}{\Lambda_{0}}\right)=-\frac{1}{K_{\nu}-1} \int_{0}^{\infty} \frac{d p}{p}\left[K_{\nu}(p)-1-\left(K_{\nu}-1\right) e^{-\Lambda_{0} p}\right] .
$$

$\Lambda_{0}$ is arbitrary but finite. Since (3.81) would be obtained by taking an exponential cutoff on $K_{\nu}(p)-1$, (3.82) amounts to finding an equivalent exponential cutoff to the cutoff of arbitrary form contained in $K_{\nu}(p)$. In the following, we assume that there is a single cutoff $\Lambda$ in the problem independent of the indices \pm and $\nu$. In the second term from $V_{+}$, the only interaction-dependent quantity is $v_{\nu}(p)$. Here it is simplest to use the fact that (3.81) can be obtained with an exponential cutoff $\Lambda$, add and subtract $\exp (-\Lambda p)$ and use $v_{\nu}$ resp. $v_{F}$ for the integrals weighted at small or large momentum. $V_{0}$ is treated in the same way. The final result is then [18, 51, 52, 53] (approximate expressions have been given by many others)

$$
\tilde{G}(x, t)=\frac{1}{2 \pi} e^{i r k_{F} x} \lim _{\alpha \rightarrow 0} \frac{\Lambda+i\left(v_{F} t-r x\right)}{\alpha+i\left(v_{F} t-r x\right)} \prod_{\nu=\rho, \sigma} \frac{1}{\sqrt{\Lambda+i\left(v_{\nu} t-r x\right)}}\left(\frac{\Lambda^{2}}{\left(\Lambda+i v_{\nu} t\right)^{2}+x^{2}}\right)^{\gamma_{\nu}}
$$

The exponent is

$$
\gamma_{\nu}=\frac{1}{8}\left(K_{\nu}+\frac{1}{K_{\nu}}-2\right) \geq 0
$$

Eq. (3.83) gives the universal behaviour of the Green function, which is independent of detailed cutoff forms. Nonuniversal contributions which have been eliminated by the trick of adding, subtracting and recombining terms above, can also be evaluated [54]. The spinless fermion result can be obtained by putting formally $K_{\rho}=K_{\sigma}=K$ and $v_{\rho}=v_{\sigma}=v$ [32, 55].

For $t=0$, the Green function decays as

$$
G_{r s}(x) \sim x^{-1-\alpha} \quad, \quad \alpha=2 \sum_{\nu} \gamma_{\nu} \geq 0
$$

The exponent $\alpha$ appears in all single-particle properties. $\alpha / 2$ is the "anomalous dimension" of the fermion operators. [It has become customary to use $\alpha$ both for the exponent of the Green function and for the infinitesimal in the bosonization identity (3.41); the 
context usually identifies clearly which $\alpha$ is referred to, and confusion seems unlikely.] From $G_{r s}$, one can derive the momentum distribution function [53, 56, 57]

$$
n(k) \sim \frac{1}{2}-C_{1} \operatorname{sign}\left(k-k_{F}\right)\left|k-k_{F}\right|^{\alpha}-C_{2}\left(k-k_{F}\right)
$$

which does not have a jump at $k_{F}$ but rather a continuous power-law variation. An exact calculation of the prefactors is also possible [53]. In (3.86) the breakdown of Fermi liquid theory and the absence of quasi-particles are evident. Fermi liquids have a jump discontinuity of amplitude $z_{k_{F}} \leq 1$ at $k_{F}$ where $z_{k}$ is the wave-function renormalization constant, Eq. (2.4). However, the velocities do not enter and charge-spin separation does not manifest itself, and only the absence of quasi-particles due to the Peierls-type coupling of the two Fermi points is probed. The single-particle density of states $N(\omega)$ varies as a power-law

$$
N(\omega) \sim|\omega|^{\alpha}
$$

with the same exponent $\alpha$. Again, exact but lengthy expressions are available [53].

The density-density correlation function consists out of several pieces, corresponding to the wave vectors $q \approx 0(\rho), q \approx \pm 2 k_{F}$ (CDW), and $q \approx \pm 4 k_{F}\left(4 k_{F}\right.$-CDW), and, in principle, higher multiples

$$
\begin{aligned}
O_{\rho}(x) & =\sum_{r, s} \rho_{r, s}(x)=\sqrt{2} \sum_{r} \rho_{r}(x)=-\frac{\sqrt{2}}{\pi} \frac{\partial \Phi_{\rho}(x)}{\partial x} \\
O_{C D W}(x) & =\sum_{s} \Psi_{+, s}^{\dagger}(x) \Psi_{-, s}(x) \\
& =\frac{1}{2 \pi \alpha} \sum_{s} U_{+, s} U_{-, s}^{\dagger} \exp \left\{-2 i k_{F} x+\sqrt{2} i\left[\Phi_{\rho}(x)+s \Phi_{\sigma}(x)\right]\right\} \\
& \approx \frac{1}{\pi \alpha} \exp \left\{-2 i k_{F} x+\sqrt{2} i \Phi_{\rho}(x)\right\} \cos \left[\sqrt{2} \Phi_{\sigma}(x)\right] . \\
O_{4 k_{F}}(x) & =\sum_{s} \Psi_{+, s}^{\dagger}(x) \Psi_{+,-s}^{\dagger}(x) \Psi_{-,-s}(x) \Psi_{-, s}(x) \\
& =\frac{2}{(2 \pi \alpha)^{2}} \exp \left\{-4 i k_{F} x+\sqrt{8} i \Phi_{\rho}(x)\right\} .
\end{aligned}
$$

In the Luttinger model, the $U_{r s}$-ladder operators give only contributions vanishing in the thermodynamic limit $L \rightarrow \infty$ and have been dropped after (3.89) [see however the remark after Eq. (3.71)]. Notice also that $O_{4 k_{F}}$ involves four fermions in the Luttinger model but, as will be explained below, is part of the (two-particle) density operator in lattice models. Moreover, the wavelength $\lambda=2 \pi / 4 k_{F}=\left(N_{0} / L\right)^{-1}$ equals the inverse particle density. Establishment of $4 k_{F}$-CDW long-range order therefore corresponds to the formation of a Wigner crystal, and we shall be interested in this possibility as well as its short-range ordered variant below. The expectation values are evaluated exactly as in the case of the Green function above, so that we only give the asymptotic decay laws

$$
\begin{aligned}
R_{\rho}(x) & =\frac{K_{\rho}}{(\pi x)^{2}}, \\
R_{C D W}(x) & \sim \cos \left(2 k_{F} x\right) x^{-2+\alpha_{C D W}} \quad, \quad \alpha_{C D W}=2-K_{\rho}-K_{\sigma}, \\
R_{4 k_{F}}(x) & \sim \cos \left(4 k_{F} x\right) x^{-2+\alpha_{4 k_{F}}} \quad, \quad \alpha_{4 k_{F}}=2-4 K_{\rho},
\end{aligned}
$$


We see that the $4 k_{F}$-correlations decay very fast at weak-coupling but become competitive with the $2 k_{F}$ ones when $K_{\rho}$ decreases [58]. For $K_{\sigma}=1,4 k_{F}$ correlations dominate over $2 k_{F}$ for $K_{\rho} \leq 1 / 3$.

The other correlation functions follow similar power-laws. Long wavelength spin fluctuations follow (3.91) with $K_{\rho} \rightarrow K_{\sigma}$. For later use, we also give the operators for the $x, y, z$-components of the SDW correlations

$$
\begin{aligned}
O_{S D W, x}(x) & =\sum_{s} \Psi_{+, s}^{\dagger}(x) \Psi_{-,-s}(x) \\
& =\frac{1}{\pi \alpha} \exp \left[-2 i k_{F} x+\sqrt{2} i \Phi_{\rho}(x)\right] \cos \left[\sqrt{2} \Theta_{\sigma}(x)\right] \\
O_{S D W, y}(x) & =-i \sum_{s} s \Psi_{+, s}^{\dagger}(x) \Psi_{-,-s}(x) \\
& =\frac{1}{\pi \alpha} \exp \left[-2 i k_{F} x+\sqrt{2} i \Phi_{\rho}(x)\right] \sin \left[\sqrt{2} \Theta_{\sigma}(x)\right] \\
O_{S D W, z}(x) & =\sum_{s} s \Psi_{+, s}^{\dagger}(x) \Psi_{-, s}(x) \\
& =\frac{i}{\pi \alpha} \exp \left[-2 i k_{F} x+\sqrt{2} i \Phi_{\rho}(x)\right] \sin \left[\sqrt{2} \Phi_{\sigma}(x)\right]
\end{aligned}
$$

The correlation functions decay as

$$
\begin{aligned}
R_{S D W}(x) & \sim \cos \left(2 k_{F} x\right) x^{-2+\alpha_{S D W}} \\
\alpha_{S D W_{x}} & =\alpha_{S D W_{y}}=2-K_{\rho}-K_{\sigma}^{-1} \quad, \quad \alpha_{S D W_{z}}=2-K_{\rho}-K_{\sigma}
\end{aligned}
$$

Singlet (SS) and triplet (TS) superconducting correlations do not oscillate and decay with exponents

$$
\begin{aligned}
\alpha_{S S} & =2-K_{\rho}^{-1}-K_{\sigma} \quad, \quad \\
\alpha_{T S 0} & =2-K_{\rho}^{-1}-K_{\sigma} \quad, \quad \alpha_{T S \pm 1}=2-K_{\rho}^{-1}-K_{\sigma}^{-1} .
\end{aligned}
$$

Each correlation function has its proper special combination of the two parameters $K_{\nu}$ in the power-law exponent which therefore parameterize completely the scaling laws between the exponents. Remember also that $K_{\nu}$ relates the three velocities for each degree of freedom, i.e. the spectrum of low-lying eigenvalues (3.32). Different is only the correlation function of the long-wavelength charge or spin fluctuations. The operator $\nu(x) \nu(0)$ is marginal with a scaling dimension -2 and does not acquire an anomalous dimension. Also its correlation function does not depend on a cutoff (in the other expressions, it has simply been suppressed), as has been discussed in Section 3.3.1.

The three components of the spin density and triplet superconductivity operators have very different representations in terms of the phase fields $\Phi_{\sigma}(x)$ and $\Theta_{\sigma}(x)$, and their correlation functions differ (at least formally) even in the exponents. This is so because our abelian bosonization scheme treats $\sigma_{z}$ on a special footing and breaks the spin-rotation symmetry $S U(2)$ down to $U(1)$. In the absence of external magnetic fields or spin-anisotropic interactions, the correlation functions must be spin-rotation invariant. We see that this requires $K_{\sigma}=1$. We shall assume this to be the case throughout this 
article except when stated to the contrary. Again, nonabelian bosonization would allow to keep the spin-rotation invariance manifest at every stage of the calculation.

The Green function's $\alpha$ is invariant under $K_{\rho} \rightarrow 1 / K_{\rho}$ and therefore does not depend in an important way on the sign of the interaction. It is positive and, had one only $g_{2}$, would be symmetric in attraction and repulsion. It is only $g_{4}$ which slightly changes the modulus of $\alpha$ when $g_{i} \rightarrow-g_{i}$. $\alpha=0$ is possible only when the fluctuations on all branches are free - the system may still be interacting, though, if $g_{4} \neq 0$. On the other hand, the manyparticle correlations do depend on the sign of the interactions: $K_{\rho}<1$ for repulsion, and $K_{\rho}>1$ for attraction. Consequently, for repulsive interactions, the $2 k_{F}$ density wave correlations decay more slowly than for free fermions $\left(\sim x^{-2}\right)$, while for attractive coupling, the superconducting correlations decay slowest. At first sight surprising will be the fact that the correlation functions of density and spin density (as well as those for singlet and triplet superconductivity) are strictly degenerate in the spin-rotation invariant Luttinger model. This is quite counterintuitive, and nature is certainly richer than such simple-minded results. On the other hand, the Luttinger liquid hypothesis requires that this degeneracy of exponents carries over to more realistic models. The resolution of this puzzle will be postponed to Chapter 4 .

If one is interested in finite temperatures, there are several possibilities. (i) One can use the conformal invariance of the Luttinger model to map the $T=0$ correlation functions onto those at $T \neq 0$. This will be demonstrated in the next section, Eq. (3.158). (ii) One can introduce Matsubara frequencies and calculate the boson propagators $\sim D_{\nu}(x \tau)$ at imaginary times. (iii) One can simply use the Bose-Einstein distribution $n_{B E}(p)$ at finite temperature in (3.77). This will decorate the integrals appearing in (3.79) and partly those in $(3.80)$ with factors $\operatorname{coth}\left(\beta v_{\nu} p / 2\right)$. The integrals can still be evaluated in terms of logarithms of Gamma functions which, for small temperatures essentially add terms $\ln \left\{\pi\left[x \pm i v_{\nu} t\right] / \sinh \left(\pi\left[x \pm i v_{\nu} t\right] / v_{\nu} \beta\right)\right\}$ to (3.81). If $x \gg v_{\nu} \beta$, the hyperbolic sine will grow exponentially, and correlation functions like (3.92) will therefore decay exponentially on a scale set by the thermal coherence length $\xi_{T}=\pi v_{F} / T$. If $\xi_{T} \gg 1 / \Lambda$, the power-laws discussed before will still show up in the window in between.

Transforming to $k$-space, one has to distinguish between the instantaneous and static correlation functions. Given a correlation function in $x$-space

$$
R_{i}(x) \sim \cos \left(n k_{F} x\right) x^{-2+\alpha_{i}} \quad, \quad R(t) \sim t^{-2+\alpha}
$$

the instantaneous and static correlations behave as

$$
R_{i}(k, t=0) \sim\left(k-n k_{F}\right)^{1-\alpha_{i}} \quad, \quad R_{i}(k, \omega=0) \sim\left(k-n k_{F}\right)^{-\alpha_{i}},
$$

respectively, with equivalent formulae for the $\omega$-dependent local and $q=0$-functions. For free fermions, the static correlations have a logarithmic divergence which is changed into a power law divergence by even weak interactions. On the other hand, the instantaneous correlations are nonsingular usually (though possibly enhanced), and singularities can only be brought up by rather strong interactions. Divergences of this kind have been observed both in computer simulations and in X-ray scattering on quasi-1D materials, and will be discussed below. 
We have not discussed charge-spin separation in detail yet. While it is contained in the full expression for the Green function (3.83), it does not influence the long-distance or time properties of the correlation functions. It is clear that this subtle feature of 1D interacting fermions can only be probed in dynamic, $q$ - and $\omega$-resolved correlation functions.

\subsection{Dynamical correlations: the spectral properties of Luttinger liquids}

Fermi liquid theory breaks down in 1D for two reasons: (i) the anomalous dimensions of the fermion operators, giving rise to the nonuniversal power laws discussed in the preceding section, and (ii) charge-spin separation. Either of them is sufficient to kill all quasi-particles in the neighbourhood of the Fermi surface, and both together will certainly cooperate. However, all correlation functions of the previous section are affected only by the anomalous dimensions. Much effort has been devoted to the study of these functions over the last decade.

On the other hand, for a long time much less has been known about the dynamical ( $x$ - and $t$ - resp. $q-$ and $\omega$-dependent) correlations. Also, how to measure charge-spin separation? Since this phenomenon is characterized by different propagation velocities for charge and spin fluctuations, fully dynamical correlation functions are needed to put it into evidence. The single-particle spectral function $\rho_{r s}(q, \omega)$ is defined as

$$
\rho_{r s}(q, \omega)=-\frac{1}{\pi} \operatorname{Im} G_{r s}(r k+q, \mu+\omega)
$$

where $G_{r s}$ is the retarded Green function (3.71), (3.83). There is no principal difficulty in computing this quantity. All we need to do is Fourier transform. This can be done quite easily for spinless fermions or for the one-branch Luttinger liquid $\left(g_{2}=0\right)$ but is laborious for the full model for $s=1 / 2$-fermions we are most interested in.

With spinless fermions we can single out the influence of the anomalous fermion dimensions. This is the generic structure [32, 53, 54, 59, 60]: At $q=0$ (i.e. $k=k_{F}$ ), $\rho(0, \omega) \sim|\omega|^{\alpha-1}$, i.e. a power-law divergence (or cusp-singularity for $\alpha>1$ ) instead of the $\delta$-function in Fermi liquid theory. Clearly, as the 1D correlations increase from zero, spectral weight is pushed away from the Fermi surface by the virtual particle-hole excitations generated by $g_{2}$. Let us increase $q$. In a Fermi liquid, the $\delta$-function would disperse with $q$ and broaden but essentially conserve its shape. In the Luttinger liquid, $\rho(q, \omega)$ strongly deforms: There is a power law singularity $\rho(q, \omega) \sim \Theta(\omega-v q)(\omega-v q)^{\gamma_{0}-1}$ at positive frequencies (for $q>0$ ) and a weaker singularity $\sim \Theta(-\omega-v q)(-\omega-v q)^{\gamma_{0}}$ at negative frequencies. In the positive frequency contribution - particle creation above the Fermi surface - spectral weight of an incoming particle is boosted to higher energies by the particle-hole excitations on both branches. The negative frequency contribution describes the destruction of particles above the Fermi surface present in the ground state as a result of particle-hole excitations. As $q$ increases, the negative frequency part is exponentially suppressed and all the spectral weight is transferred to positive frequencies. 
For the "one-branch" Luttinger liquid ( $g_{2}=0$, charge-spin separation only), one has finite spectral weight only at positive frequencies (for $q>0$ ) between $v_{\sigma} q$ and $v_{\rho} q$ with inverse-square-root divergences at the edges [53, 59, 61]. At $k_{F}$, the spectral function reduces to $\delta(\omega)$ and the momentum distribution is a step function with a jump of unity at $k_{F}$, in agreement with Luttinger's theorem [4]. Although this seems to imply a Fermi liquid it is clear that the physical picture is quite different and that the notion of a quasi-particle does not make sense because the $\delta$-function does not survive the slightest displacement from the Fermi surface. The incident electron decays into multiple particle-hole-like charge and spin fluctuations which all live on the same branch as the incoming fermion. It is immediately apparent that $n(k)$ and, more generally, any quantity depending on $k$ or $\omega$ alone will fail to detect charge-spin separation. It can be seen only in quantities depending on both $q$ and $\omega$.

We now turn to the spectral properties of the $s=1 / 2$-Luttinger liquid [53, 54, 59, 60, 62. We limit ourselves to the spin-rotation invariant case $\left(\gamma_{\sigma}=0\right)$. Fig. 3.6 displays the dispersion of $\rho(q, \omega)$ for small $q$ and $\alpha=0.125$. It is apparent that the spectral function carries features both from the spinless fermions (synonymous with "anomalous fermion dimensions") and the one-branch problem ("charge-spin separation"). At very small $q$, on the scale of the Figure, $\rho$ looks pretty much like the spinless fermions' function. As $q$ increases, the negative frequency weight (very small anyway) is transferred to positive frequency but, most importantly, the generic two-peak structure of the spectral function becomes apparent. The exponent of the singularity at $v_{\sigma} q$ is $2 \gamma_{\rho}-1 / 2$ while it is $\gamma_{\rho}-1 / 2$ at the $v_{\rho} q$-singularity and $\gamma_{\rho}$ at $-v_{\rho} q$. Since $\gamma_{\rho}=1 / 16$ here, the correction to the onebranch case is quite insignificant here and the charge-spin separation aspect is clearly dominant at finite $q$. The weight above/below $\pm v_{\rho} q$ originating from the anomalous dimensions is barely visible. As $\alpha$ increases, the various power-law divergences weaken and finally transform into cusp-singularities. At the same time, the spectral function becomes much less structured, and spectral weight is shifted by the electronic correlations both to above/below $\pm v_{\rho} q$, more reminiscent of the spinless fermion problem. As the correlations increase, the features originating from charge-spin separation are more and more obscured by transfers of spectral weight over significant energy scales. The important scale here is the energy of the charge fluctuations $\pm v_{\rho} q$.

The spectral function in Figure 3.6 obeys to the sum rule

$$
\int_{-\infty}^{\infty} d \omega \rho_{r s}(q, \omega)=1 \text { for all } q
$$

The single-particle density of states $N(\omega)$ has already been discussed above. A local sum rule is not satisfied by $N(\omega)$ unless $g_{4 \|}=0$ [51] as is the case for local interactions; in general (long-range interactions), one has [53, 62, 63.

$$
\int_{0}^{\infty} d \omega\left[N_{r s}(\omega)-N_{0}(\omega)\right]=-\frac{1}{4 \pi v_{F}} \int_{-\infty}^{\infty} \frac{d k}{2 \pi} g_{4 \|}(k)
$$

$N_{0}(\omega)=1 / 2 \pi v_{F}$ being the noninteracting density of states. It is satisfied, however, by the Tomonaga model with a finite bandwidth cutoff 63 . Here, as usual, $\int_{0}^{\infty} d \omega N(\omega)=n$, 
the particle density which is not changed by the interactions. The failure of the local sum rule in the Luttinger model is certainly due to the introduction of the unphysical negative-energy states which are sampled in the frequency integral.

The many-particle spectral functions display similar features. Fig. 3.7 displays the charge $[S(q, \omega)]$ and spin $[\chi(q, \omega)]$ structure factors at $2 k_{F}$ and the charge factor at $4 k_{F}$ $\left[S_{4}(q, \omega)\right]$ [53, 64]. Again there are power-law singularities at $\omega= \pm v_{\sigma} q$ and $\pm v_{\rho} q$ but the functions now are symmetric because the CDW and SDW operators mix left- and right-moving particles. At weak coupling, there are cusps, and only as $K_{\rho}<1 / 2$ do they turn into divergences. Further interesting is the fact that the $2 k_{F}$ CDW and SDW fluctuations are sensitive to charge-spin separation but the $4 k_{F^{-}} \mathrm{CDW}$ is not. This is easy to understand from the boson representation of these operators (3.89) - (3.96): the $2 k_{F^{-}}$ operators necessarily involve the $\Phi_{\sigma}$ or $\Theta_{\sigma}$-fields in addition to $\Phi_{\rho}$. The only divergent $4 k_{F}$-operator, however, only depends on the charge field $\Phi_{\rho} .4 k_{F}$-operators involving the spin degrees of freedom are never divergent.

\subsection{Alternative methods}

\subsubsection{Green function methods}

There are alternative routes for solving the Tomonaga-Luttinger model, based on diagrammatic methods or equations of motion for the Green function. They provide an interpretation of the novel physics of the Luttinger liquid from the standpoint of conventional many-body theory, and therefore stress the formal similarities of Fermi and Luttinger liquids while the bosonization approach more strongly emphasizes their differences. Moreover, the connection between symmetries, conservation laws and the lowenergy structure of 1D Fermi liquids may become more apparent in this approach which we outline now. It has been pioneered by Dzyaloshinskii and Larkin for a spinless variant of the model, Eq. (3.52] [34] and followed and extended by others [18, 35, 46, 65].

The power of the 1D conservation laws can be gauged from the fact that our arguments for the breakdown of Fermi liquid theory in 1D in Section 2.1 were based on divergences encountered in a perturbation treatment of the self-energy corrections to the 1D Green functions. As a consequence of Ward identities, vertex and self-energy correction cancel exactly in some quantities (such as density-density correlation functions) and to such a large extent in others that meaningful answers are obtained and all results of the bosonization approach reproduced.

What are Ward identities? They are specific relations between the vertex operators and (single or $n$-particle) Green functions of a theory, translating its conservation laws i.e. its symmetries, into a Green function formalism which describes the dynamics of the excitations. Vertex operators couple the charges and currents of a system to external fields. They involve the corresponding density operator (e.g. $\rho(p), j_{\rho}(p)$ ) plus two (more generally $2 n$ ) fermions. The equation of motion for the vertex operator in general produces Green functions involving even more particles. If the charge is conserved, however, $\rho(p)$ obeys the continuity equation. Combining it with the equation of motion of the simple 
vertex described, yields just the difference of the two single-particle propagators involved, instead of complicated objects involving intermediate excitations. The principle is easy: use the continuity equation associated with the conserved charge to reduce the equations of motion for the object under consideration, then Fourier transform the resulting expression to recover an algebraic relation. This is particularly transparent for density-density response which we study now before carrying on to the single-particle Green function.

In Section 3.1.3, we had studied the conservation of charge and spin separately on each branch of the dispersion. This generates the following continuity equations for the charge and spin densities and currents from the Heisenberg equations of motion

$$
\begin{aligned}
i \frac{\partial \nu(p, t)}{\partial t} & =[\nu(p, t), H]=-v_{J_{\nu}} p j_{\nu}(p, t) \\
i \frac{\partial \tilde{\nu}(p, t)}{\partial t} & =[\tilde{\nu}(p, t), H]=-v_{N \nu} p \tilde{j}_{\nu}(p, t)
\end{aligned}
$$

with the total charge $(\nu=\rho)$ and spin $(\nu=\sigma)$ densities and currents

$$
\nu(p)=\sum_{r} \nu_{r}(p) \quad \text { and } \quad j_{\nu}(p)=\sum_{r} r \nu_{r}(p)
$$

In the Green function approach, it is the physical charge and spin densities which enter the various operators. For this reason, we define in this section, and only in this section

$$
\nu_{r}(p)=\rho_{r \uparrow}(p) \pm \rho_{r \downarrow}(p)
$$

at variance with the remainder of this paper. This will avoid a confusing proliferation of factors $\sqrt{2}$ due to the different definition in (3.22). The "axial" charge and spin densities and currents (named after similar constructions appearing in field theory) are

$$
\tilde{\nu}(p)=\sum_{r} r \nu_{r}(p) \text { and } \tilde{j}_{\nu}(p)=\sum_{r} \nu_{r}(p)
$$

and are identical to the usual currents and charges, Eq. (3.108), respectively. $v_{J \nu}$ and $v_{N \nu}$ are the velocities for charge and current excitations [3] defined earlier (3.32).

Notice in passing that both equations can be put together to produce the equations of motion of a harmonic oscillator 46]

$$
\frac{\partial^{2} \nu(p, t)}{\partial t^{2}}+v_{J \nu} v_{N \nu} p^{2} \nu(p, t)=0
$$

indicating that the charge (spin) density fluctuations are the elementary excitations of the systems which propagate with an effective (sound) velocity $\sqrt{v_{J \nu} v_{N \nu}}$. The conservation laws thus completely determine the dynamics of our system.

For illustration, we investigate the density-density correlation function

$$
R_{\rho \rho}(q, \omega)=\int_{-\infty}^{\infty} d t e^{i \omega t} R_{\rho \rho}(q, t) \quad, \quad R_{\rho \rho}(q, t)=-\frac{i}{L}\langle T \rho(q, t) \rho(-q, 0)\rangle .
$$

$T$ is the time ordering operator. Applying $i \partial_{t}$ to this equation and using (3.106), we have

$$
i \frac{\partial R_{\rho}(q, t)}{\partial t}=\frac{i}{L} v_{J \rho} q\left\langle T j_{\rho}(q, t) \rho(-q, 0)\right\rangle+\frac{1}{L} \delta(t)\langle[\rho(q), \rho(-q)]\rangle
$$


where the last term originates from taking the time derivatives of the step functions implied by time ordering but vanishes on account of the commutator algebra Eq. (3.17). A first Ward identity is obtained from the Fourier transform

$$
\omega R_{\rho \rho}(q, \omega)-v_{J \rho} q R_{j_{\rho} \rho}(q, \omega)=0
$$

Similar Ward identities can be derived for $R_{j_{\rho} \rho}(q, \omega)$ (with the difference that $\left[j_{\rho}(q), \rho(q)\right]$ $\neq 0$ ) and for the axial charges and currents (3.110). The second derivative of the densitydensity correlation function is then

$$
-\frac{\partial^{2} R_{\rho \rho}(q, t)}{\partial t^{2}}=v_{J \rho} v_{N \rho} q^{2} R_{\rho \rho}(q, t)+\frac{2 v_{J \rho} q^{2}}{\pi} \delta(t)
$$

which is Fourier transformed into

$$
R_{\rho \rho}(q, \omega)=\frac{2}{\pi} \frac{v_{J \rho} q^{2}}{\omega^{2}-v_{\rho}^{2} q^{2}} \quad \text { with } \quad v_{\rho}=\sqrt{v_{J \rho} v_{N \rho}}
$$

Eq. (3.113) is an example of a very simple - yet manifestly powerful - Ward identity. Metzner and Di Castro [46] give many more.

Now consider the single-particle Green function

$$
G_{r s}(k, t)=-i\left\langle T c_{r s}(k, t) c_{r s}^{\dagger}(k, 0)\right\rangle
$$

which obeys the equation of motion [35]

$$
\begin{aligned}
\left(\omega-r v_{F} k\right) G_{r s}(k, \omega)= & 1+i \sum_{\nu, q} \int \frac{d \Omega}{2 \pi}\left[g_{2 \nu}\left(1-2 \delta_{\nu, \sigma} \delta_{s, \downarrow}\right) F_{-r r s}^{\nu}(k, \omega ; k+q, \omega+\Omega ; q, \Omega)\right. \\
& \left.+g_{4 \nu}\left(1-2 \delta_{\nu, \sigma} \delta_{s, \downarrow}\right) F_{r r s}^{\nu}(k, \omega ; k+q, \omega+\Omega ; q, \Omega)\right]
\end{aligned}
$$

To get (3.118), take $i \partial_{t} G_{r s}(k, t)$ using the Heisenberg equation of motion and Fourier transform; deriving the $T$-operator gives the 1 , taking the commutator with $H_{0}$ gives $r v_{F} k G_{r s}$, and the commutators with $H_{2}$ and $H_{4}$ and using (3.22) to go from the $\rho_{r s}$ to the $\nu_{r}$, gives the vertex functions

$$
F_{r^{\prime} r s}^{\nu}\left(k_{1}, t_{1} ; k_{2}, t_{2} ; q t\right)=-\left\langle T \nu_{r^{\prime}}(q t) c_{r s}\left(k_{2}, t_{2}\right) c_{r s}^{\dagger}\left(k_{1}, t_{1}\right)\right\rangle
$$

Continuing now without using (3.106) would lead to a hopeless hierarchy of equations. However, (3.119) obeys a remarkable Ward identity

$q F_{r^{\prime} r s}^{\nu}(k, \omega ; k+q, \omega+\Omega ; q, \Omega)=r \pi\left(1-2 \delta_{\nu, \sigma} \delta_{s, \downarrow}\right) R_{\nu_{r^{\prime} \nu_{r}}}(q, \Omega)\left[G_{r s}(k, \omega)-G_{r s}(k+q, \omega+\Omega)\right]$

which helps to simplify the problem. The one-branch density-density correlation function

$$
R_{\nu_{r^{\prime}} \nu_{r}}(q, \omega)=-\frac{i}{L} \int d t e^{i \omega t}\left\langle T \nu_{r^{\prime}}(q t) \nu_{r}(-q 0)\right\rangle= \begin{cases}\frac{r q}{\pi} \frac{\omega+r\left(v_{N \nu}+v_{J \nu}\right) q / 2}{\omega^{2}-\left(v_{\nu} q\right)^{2}} & \text { for } r=r^{\prime} \\ \frac{1}{\pi} \frac{\left(v_{J \nu}-v_{N \nu}\right) q^{2} / 2}{\omega^{2}-\left(v_{\nu} q\right)^{2}} & \text { for } r=-r^{\prime}\end{cases}
$$


can itself be derived from (3.113) and related Ward identities. One can now eliminate the vertex function from (3.118) and close the equation of motion for the Green function. The resulting integral equation is then solved by Fourier transforming back to a real space differential equation and taking into account boundary and analyticity conditions. The result agrees with the expression (3.83) up to details of cutoff procedures. Notice that the Ward identity for $F_{r^{\prime} r s}^{\nu}$ (3.120) involves the chiral (charge and spin) density operators $\nu_{r}$. It therefore is the consequence of two separate Ward identities, one for the density $\sum_{r} \nu_{r}$ which is present also in the many-body problem in higher dimensions, and a new one involving the axial density $\sum_{r} r \nu_{r}$ which is new and related to the disconnected 1D Fermi "surface" and the absence of backscattering in the Luttinger model.

In these two examples, we have rederived results via Ward identities which are also quite easy to derive from bosonization. There are others where the derivation via Ward identities are easier than with bosonization. An example is the intra- or inter-branch polarization bubble $\Pi_{r r^{\prime}}^{\rho}(q, \omega)$ which is related to the density correlation function (3.121) by Dyson's equation

$$
R_{\rho_{r} \rho_{r^{\prime}}}(q, \omega)=\Pi_{r r^{\prime}}^{\rho}(q, \omega)+\sum_{t t^{\prime}} \Pi_{r t}^{\rho}(q, \omega) g_{t t^{\prime} \rho} R_{\rho_{t^{\prime}} \rho_{r^{\prime}}}(q, \omega)
$$

represented graphically in Figure 3.4. $g_{r r^{\prime} \rho}$ denotes $g_{2 \rho}$ or $g_{4 \rho}$. The polarization $\Pi_{r r^{\prime}}^{\rho}$ is given by the irreducible vertex $\Lambda_{r r^{\prime} s}^{\rho}$ and the exact single-particle Green functions $G_{r^{\prime} s}$

$$
\Pi_{r r^{\prime}}^{\rho}(q \omega)=-i \sum_{s} \int \frac{d k d \Omega}{(2 \pi)^{2}} \Lambda_{r r^{\prime} s}^{\rho}(k, \Omega ; k+q, \omega+\Omega ; q, \omega) G_{r^{\prime} s}(k, \Omega) G_{r^{\prime} s}(k+q, \Omega+\omega)
$$

as shown in Fig. 3.5. $\Lambda$ is obtained from $F$ by amputating the external fermion legs, i.e. dividing by the product of the two Green functions involved in (3.120) and taking only the interaction-irreducible part of $F$. $\Pi$ must be a wildly divergent function because the Green functions have divergences and the vertex corrections certainly have divergences, too! This is not true, however, and with the Ward identity (3.120), converted into one for $\Lambda$, one obtains the simple, finite results

$$
\begin{aligned}
\Pi_{r,-r}^{\rho}(q, \omega) & =0 \\
\Pi_{r r}^{\rho}(q, \omega) & =\frac{-i}{\omega-r v_{F} q} \sum_{s} \int \frac{d k d \Omega}{(2 \pi)^{2}}\left[G_{r s}(k, \Omega)-G_{r s}(k+q, \Omega+\omega)\right] \\
& =\frac{r}{\pi} \frac{q}{\omega-v_{F} q} \equiv \Pi_{r r}^{\rho(0)}(q, \omega) .
\end{aligned}
$$

This result is remarkable: all vertex and self-energy corrections have cancelled out as a consequence of the Ward identities, and the polarization is identical to $\Pi^{\rho(0)}$ of free fermions. Eq. (3.122) then reduces to a standard RPA summation, showing that RPA is exact for the density-density correlation functions. Moreover, the charge and spin susceptibilities $\lim _{q \rightarrow 0} \lim _{\omega \rightarrow 0} R_{\nu \nu}(q, \omega)$ are finite, in agreement with (3.62). The Luttinger liquids therefore are "normal" metals. This is entirely due to the conservation laws and Ward identities which enforce the cancellation of all divergences which would occur in a diagrammatic development. 
Of course, one can also compute all the many-particle Green functions, and construct the same picture as in the preceding sections using the standard many-body formalism. We reemphasize that in the exact solutions we had found, both the Ward identities related to the charges (currents) and to the axial charges (currents) were essential. It is the latter one that gives the one-dimensional Fermi liquids their special properties.

Similar results can also be obtained by more diagram-based techniques [18, 34, 65]. In this case, the Ward identities are expressed by the theorem that closed fermion loops with more than two fermion lines vanish (equivalent in the vanishing of the transverse current in quantum electrodynamics). The limitation to forward scattering only in the Luttinger model implies that a closed fermion line has all of its parts on a definite branch $r$.

Moreover, one can use the Ward identities to construct a field-theoretical renormalization group formulation of the Luttinger model with respect to the free Fermi gas [46, 66]. This verifies that all couplings are dimensionless, and that consequently, the beta-function

$$
\beta(g)=\Lambda\left(\frac{\partial g}{\partial \Lambda}\right) \equiv 0
$$

at the Luttinger liquid fixed point. The density operators do not acquire anomalous dimensions, and the coupling constants are renormalization group invariants. It also verifies the correctness of the earlier scaling Ansatz [18].

\subsubsection{Other bosonic schemes}

In Section 3.2.2, we have solved the Luttinger model via a boson representation of the Hamiltonian and of the fermion operators. Other bosonic approaches, based on functional integrals and a Hubbard-Stratonovich decoupling have been developed in the past [61, 67]. They are closer to the methods used in quantum field theory than Haldane's operator approach. They also provide an exact solution of the model, and reproduce all the results obtained by the two methods presented above. Which one to use is rather a matter of taste and background than of the specific nature of the problem at hand.

A bosonic scheme widely used for strongly correlated fermions are "slave bosons" and one may naturally wonder if there is any relation to the bosonization discussed above. Slave bosons are usually applied to problems where double occupancy of lattice sites is dynamically forbidden because of strong electronic repulsion. One tries to circumvent the difficult treatment of inequality constraints (such as $\left\langle n_{i}\right\rangle \leq 1$ ) by introducing additional particles into an enlarged Hilbert space whereby the inequality constraint translates into an equality constraint which can be solved by Lagrange multipliers. Properties are then obtained by projecting back onto the physical Hilbert space. From these remarks, it is quite clear that slave bosons and the Tomonaga-Luttinger bosons are two distinct entities. While the latter are the elementary excitations of the 1D Fermi liquid, the former are, in the first place, a bookkeeping device to obtain good approximations to fermionic properties. Still, slave bosons have been used successfully, together with standard bosonization, to obtain low-energy properties of, e.g., the $U=\infty$ Hubbard model [68]. A deeper knowledge of differences and similarities of both types of bosons is, however, just beginning to emerge 69 . 


\subsection{Conformal field theory and bosonization}

In the language of the theory of phase transitions, one-dimensional Fermi liquids are critical at $T=0$. An arbitrary system, close to a second order phase transition, exhibits strong precursor fluctuations of the ordered phase, whose typical size is measured by the correlation length $\xi \sim\left|\left(T-T_{c}\right) / T_{c}\right|^{-\nu}$ which diverges as the critical point $T_{c}$ is approached. Thermodynamic properties (specific heat, magnetization, etc.) exhibit similar divergences whose sole origin is the divergence in $\xi$. Therefore, their critical exponents can be related by scaling relations to $\nu$ and the dimension of space. These scaling relations only depend on the symmetry of the theory (universality). At the critical point, correlation functions decay as power-laws of distance and time with some critical exponents which generally can be calculated from the model under consideration [70]. The power-law correlations of one-dimensional Fermi liquids found in Section 3.3, show explicitly that we have a $T=0$ quantum critical point.

\subsubsection{Conformal invariance at a critical point}

Conformal field theory is a powerful means of characterizing universality classes of critical systems in 2D statistical mechanics or 1D quantum field theories [time playing the role of a second dimension, these theories in fact are $(1+1) \mathrm{D}]$ in terms of a single dimensionless number, the central charge $c$ of the underlying Virasoro algebra [29]. The critical exponents are the scaling dimensions of the various operators in a conformally invariant theory and, generically, are fully determined by $c$. A notable exception are theories with central charge $c=1$ such as the Gaussian model, of particular relevance to the problems considered here, where the exponents (scaling dimensions) depend on a single effective coupling constant of the model. Both the central charge and the scaling dimensions can be computed from the finite-size scaling properties of the ground state energy and the low-lying excitations [29, 71]. This is important because these quantities can be computed accurately either by Bethe Ansatz (for models solvable by the technique) or, in any case, by numerical diagonalization.

What are the symmetries of systems at a critical point? It is certainly translationally and rotationally invariant. Quantum field theories, in addition are Lorentz invariant but in $(1+1) \mathrm{D}$, Lorentz invariance reduces to rotations in the $\mathbf{x}=(x, t)$-plane. As we have seen above, a system at criticality, in addition is characterized by scale invariance,

$$
\mathbf{x} \rightarrow \lambda \mathbf{x}
$$

It turns out that the combined rotational and scale invariance implies that the system is invariant under a wider symmetry group, the global conformal group. On a classical level, conformal transformations are general coordinate transformations which leave the angles between two vectors invariant. In dimension $D>2$, the global conformal group is finite-dimensional, and so is the associated Lie algebra of its generators. There is a finite number of constraints, and these allow for an evaluation of the two-point and three-point correlation functions, but not for the higher ones. 
The situation is different in two dimensions, where all correlation functions can be determined. Consider a general coordinate transformation

$$
\mathbf{x} \rightarrow \mathbf{x}^{\prime}=\mathbf{x}+\xi(\mathbf{x})
$$

For this transformation to be conformal, $\xi$ must satisfy certain constraints which can be expressed in a differential equation (Killing-Cartan equation). In general dimension $D$, this leaves for $\xi(\mathbf{x})$ a polynomial of second degree in $\mathbf{x}$ (with tensor coefficients). In two dimensions, however, the Killing-Cartan equation reduces to the Cauchy-Riemann equation, and therefore all analytic functions are allowed for conformal transformations. This group of transformations, called local conformal group, is much wider than the global conformal group encountered before. It is then natural to switch to complex variables $z, \bar{z}=x_{1} \pm i x_{2}$, so that we have

$$
z \rightarrow z+\xi^{z}(z)=f(z) \quad, \quad \bar{z} \rightarrow \bar{z}+\bar{\xi}^{\bar{z}}(\bar{z})=\bar{f}(\bar{z})
$$

To determine the algebra corresponding to the local conformal group, we need the commutation relations of the generators of the transformations. Since $\xi^{z}(z)$ and $f(z)$ are analytic, they can be expanded in a Laurent series

$$
\xi^{z}(z)=\sum_{n=-\infty}^{\infty} \xi_{n} z^{n+1}
$$

[and a similar equation for $\bar{\xi}(\bar{z})]$, and we find the generators of the local conformal transformations

$$
\ell_{n}(z)=-z^{n+1} \partial_{z}, \quad \bar{\ell}_{n}(\bar{z})=-\bar{z}^{n+1} \partial_{\bar{z}}, \quad n \in \mathbb{Z}
$$

These generators obey the local conformal algebra

$$
\left[\ell_{m}, \ell_{n}\right]=(m-n) \ell_{m+n}, \quad\left[\bar{\ell}_{m}, \bar{\ell}_{n}\right]=(m-n) \bar{\ell}_{m+n}, \quad\left[\ell_{m}, \bar{\ell}_{n}\right]=0
$$

This infinite dimensional algebra is called the classical Virasoro algebra. (The global conformal algebra is generated by $\left\{\ell_{-1}, \ell_{0}, \ell_{1}\right\}$.) Since the two algebras are independent, one may take $z$ and $\bar{z}$ as independent, corresponding to the natural variables for left- and right-moving objects; the physical theory then lives on $\bar{z}=z^{\star}$.

We now go to the quantum (or statistical mechanics) case. How do fields and correlation functions of a quantum field theory transform under conformal transformations? In general, an infinitesimal symmetry variation in a field $\phi$ is generated by $\delta_{\xi} \phi=\xi[Q, \phi]$ where $Q$ is the conserved charge associated with the symmetry. Local coordinate transformations are generated by the charges constructed from the stress-energy tensor $T_{i j}$. Rotational invariance constrains $T_{i j}$ to be symmetric, and scale invariance requires its trace to vanish; then conformal invariance does not impose additional constraints showing that it is implied by rotational and dilatational invariance. Translating these conditions into the complex variables $z$ and $\bar{z}$, one can show that only the diagonal components

$$
T(z) \equiv T_{z z}(z) \quad \text { and } \quad \bar{T}(\bar{z}) \equiv \bar{T}_{\bar{z} \bar{z}}(\bar{z})
$$


do not vanish. In the radial quantization scheme, the conserved charge then becomes

$$
Q=\frac{1}{2 \pi i} \oint[d z T(z) \xi(z)+d \bar{z} \bar{T}(\bar{z}) \bar{\xi}(\bar{z})],
$$

which generates a field variation

$$
\delta_{\xi, \bar{\xi}} \phi(w, \bar{w})=\frac{1}{2 \pi i} \int\{d z[T(z) \xi(z), \phi(w, \bar{w})]+d \bar{z}[\bar{T}(\bar{z}) \bar{\xi}(\bar{z}), \phi(w, \bar{w})]\} \quad .
$$

In general, it is difficult at this point to proceed further without having explicit expressions at hand. There is, however, a distinctive class of fields, to be called primary fields, for which

$$
\delta_{\xi, \bar{\xi}} \phi(w, \bar{w})=\left(h \partial_{z} \xi^{z}(z)+\xi^{z}(z) \partial_{z}+\bar{h} \partial_{\bar{z}} \bar{\xi}^{\bar{z}}(\bar{z})+\bar{\xi}^{\bar{z}}(\bar{z}) \partial_{\bar{z}}\right) \phi(w, \bar{w})
$$

which can be recognized as the infinitesimal version of

$$
\phi(w, \bar{w}) \rightarrow\left(\frac{\partial f}{\partial w}\right)^{h}\left(\frac{\partial \bar{f}}{\partial \bar{w}}\right)^{\bar{h}} \phi(f(w), \bar{f}(\bar{w}))
$$

All other fields are called secondary fields. $h$ and $\bar{h}$ are two real numbers, the conformal weights of the field $\phi$. The combinations $\Delta=h+\bar{h}$ and $s=h-\bar{h}$ are the scaling dimension and spin of the field $\phi$, respectively [if one works in a basis of eigenstates of $L_{0}$ and $\bar{L}_{0}$, the combinations $L_{0}+\bar{L}_{0}$ and $i\left(\mathrm{E}_{0}-\bar{L}_{0}\right)$ are generators of dilations and rotations, respectively]. Eq. (3.137) is the transformation law of a complex tensor of rank $h, \bar{h}$. Normally, such a tensor transforms with integer powers of $\partial f / \partial z$ and $\partial \bar{f} / \partial \bar{z}$ which are the number of $z$ and $\bar{z}$ indices; here, however, one could conceive also noninteger exponents. They are called anomalous dimensions. As a consequence, the scaling dimension of the field $\phi$ also can become anomalous. We have seen examples in the Luttinger model in the preceding section.

One reason for the special status of primary fields is that one can derive (in fact in any dimension) some of their correlation functions from the transformation property (3.136). The two-point function $G^{(2)}=\left\langle\phi_{1}\left(z_{1}, \bar{z}_{1}\right) \phi_{2}\left(z_{2}, \bar{z}_{2}\right)\right\rangle$ must be invariant under a conformal transformation $(3.129)$

$$
\delta_{\xi, \bar{\xi}} G^{(2)}\left(z_{i}, \bar{z}_{i}\right)=\left\langle\left(\delta_{\xi, \bar{\xi}} \phi_{1}\right) \phi_{2}\right\rangle+\left\langle\phi_{1} \delta_{\xi, \bar{\xi}} \phi_{2}\right\rangle=0
$$

Using the transformation law (3.136), one can derive a differential equation for $G^{(2)}$ which can be solved to yield

$$
G^{(2)}\left(z_{i}, \bar{z}_{i}\right)=\frac{C_{12}}{z_{12}^{2 h} \bar{z}_{12}^{2 \bar{h}}}
$$

where $z_{i j}=z_{i}-z_{j}$ and $C_{12} \propto \delta_{\Delta_{1}, \Delta_{2}}$ is a constant. The three-point function $G^{(3)}$ can be determined in a similar manner, but the four-point function, at the present stage of development, can only be determined up to a function of the cross-ratio $z_{12} z_{34} / z_{13} z_{24}$.

Not all fields are primary fields. For the primary fields to transform according to (3.136), the operator product expansion (OPE) of the stress-energy tensor with $\phi$ for short distances must go as

$$
T(z) \phi(w, \bar{w})=\frac{h}{(z-w)^{2}} \phi(w, \bar{w})+\frac{1}{z-w} \partial_{w} \phi(w, \bar{w})+\ldots
$$


where radial ordering is implied, and there is an equivalent equation for the anti-holomorphic (left-moving) piece of the stress-energy tensor. (In the following, we always imply the existence of such equivalent equations for the anti-holomorphic dependences.) A secondary field has a higher than double-pole singularity in its OPE with $T(z)$. The most prominent representative is $T(z)$ itself

$$
T(z) T(w)=\frac{c / 2}{(z-w)^{4}}+\frac{2}{(z-w)^{2}} T(w)+\frac{1}{z-w} \partial T(z) .
$$

The coefficient $c(=\bar{c} \geq 0)$ is called the central charge. It cannot be determined by the requirement of conformal invariance alone, and will depend on the theory studied. Different values of $c$ will imply different universality classes.

The nonvanishing of $c$ represents an anomaly which often occurs in problems with local symmetries. It means that a classical symmetry cannot be implemented quantummechanically due to renormalization effects. Therefore not all fields but only the primary fields transform according to (3.136). As will be seen below, $T(z)$ determines the change in action under a local coordinate transformation. In a path-integral formalism, the anomaly in $T$ then implies that the complete measure cannot be made conformally invariant. The anomaly is also called Schwinger term. As examples, for a free boson $\phi(z), T(z)=$ : $\left[\partial_{z} \phi(z)\right]^{2}: / 2$ and $c=1$; free real (Majorana) fermions $\psi(z)$, relevant for the 2D Ising model, have $T(z)=: \psi(z) \partial_{z} \psi(z): / 2$ and $c=1 / 2$; finally, free complex (Dirac) fermions $\Psi(z)$, relevant for the Luttinger model, have $T(z)=i:\left[\partial_{z} \Psi^{\dagger}(z)\right] \Psi(z)-\Psi^{\dagger}(z) \partial_{z} \Psi(z): / 2$ and $c=1$ like the bosons.

This anomaly has important consequences for the algebra of the generators of the local conformal transformations on the quantum level. Just as above on the classical level, one can derive the algebra of the generators from a Laurent expansion of the stress-energy tensor

$$
T(z)=\sum_{n=-\infty}^{\infty} L_{n} z^{-n-2} .
$$

Using (3.141), we obtain the Virasoro algebra with central extension $c$

$$
\begin{aligned}
{\left[L_{n}, L_{m}\right] } & =(n-m) L_{n+m}+\frac{c}{12}\left(n^{3}-n\right) \delta_{n+m, 0} \\
{\left[\bar{L}_{n}, \bar{L}_{m}\right] } & =(n-m) \bar{L}_{n+m}+\frac{\bar{c}}{12}\left(n^{3}-n\right) \delta_{n+m, 0} \\
{\left[L_{n}, \bar{L}_{m}\right] } & =0
\end{aligned}
$$

The classical Virasoro algebra is recovered for $c=0$. Every conformal quantum field theory defines a representation of (3.143) with some central charge $c, \bar{c}$. The $L_{n}$ are the generators of transformations of quantum fields associated with the monomial of degree $n+1$ in $z$. For $\xi^{z}(z)=-\xi_{n} z^{n+1}$, we have

$$
\delta \phi(z, \bar{z})=-\xi_{n}\left[L_{n}, \phi(z, \bar{z})\right] .
$$

Unitarity constrains the generators to satisfy

$$
L_{m}^{\dagger}=L_{-m}
$$


and regularity of the stress-energy tensor at the origin implies

$$
L_{m}|0\rangle=0, \quad m \geq-1 \quad \text { and } \quad L_{m}^{\dagger}|0\rangle=0, \quad m \leq-1
$$

in their action on the vacuum $|0\rangle$.

There are two more important properties of the stress-energy tensor. Under a local conformal transformation to $z^{\prime}=f(z)$, it transforms as

$$
\begin{aligned}
T(z) & \rightarrow T^{\prime}(z)=\left(\frac{d z^{\prime}}{d z}\right)^{2} T\left(z^{\prime}\right)+\frac{c}{12}\left\{z^{\prime}, z\right\} \\
\left\{z^{\prime}, z\right\} & =\frac{\partial_{z}^{3} z^{\prime}}{\partial_{z} z^{\prime}}-\frac{3\left(\partial_{z}^{2} z^{\prime}\right)^{2}}{2\left(\partial_{z} z^{\prime}\right)^{2}}
\end{aligned}
$$

The first term in (3.147) translates the fact that $T(z)$ is a field of conformal weight $(2,0)$ in agreement with (3.141) above, while the second term contains the conformal anomaly. (3.148) is known as the Schwarzian derivative.

We now turn to the representations of the Virasoro algebra, i.e. the states of our Hilbert space. In general, the representations of symmetry groups are constructed from highest weight vectors (states). Such a highest weight state $|h\rangle$ is created by the action of a holomorphic primary field $\phi$ on the vacuum, at the origin

$$
|h\rangle=\phi(0)|0\rangle \quad, \quad L_{0}|h\rangle=h|h\rangle \quad, \quad L_{n}|h\rangle=0 \quad, \quad n>0 \quad .
$$

$|h\rangle$ is thus eigenstate of $L_{0}$. The $L_{n}, n>0$ are the lowering operators annihilating $|h\rangle$. The corresponding raising operators are $L_{-n}, n>0$ and, acting on $|h\rangle$, generate the descendant states

$$
L_{-n_{1}} \ldots L_{-n_{k}}|0\rangle \neq 0 \quad, \quad 1 \leq n_{1} \leq \ldots \leq n_{k}
$$

They form a basis for the representation vector space. The eigenvalue of $L_{0}$ on the state (3.150) is $h+n_{1}+\ldots+n_{k}$. The highest weight state $|h\rangle$ has the lowest eigenvalue among all the states that can be created out of it by acting with the raising operators. It is the ground state in a given sector of the theory. The descendants are the excited states. The $L_{n}(n>0)$ act as an infinite number of harmonic oscillator annihilation operators, and the $L_{n}^{\dagger}=L_{-n}$ then are the creation operators. The level of the state (3.150) is $\sum_{i=1}^{k} n_{k}$, and the level associated with an operator $L_{-n}$ is $n$. The number of basis vectors on a given level $N$ is $P(N)$, the number of partitions of $N$. The conformal weight of all the descendant states on level $N$ is $h+N$. The vector space generated from $|h\rangle$ is called Verma module.

All states (and fields) in a conformal field theory can be grouped into conformal families (towers). They consist of a highest weight state $|h\rangle$ and all the descendant states generated by the application of the raising operators $L_{-n}$. The different highest weight states are obtained from the action of the different primary fields $\phi_{n}(z)$ [or, more generally, $\phi_{n}(z, \bar{z})$ ] on the vacuum according to (3.149). The conformal families offer a very convenient way to classify the excitations in the system and the spectrum of the scaling dimensions. 
All correlation functions involving secondary fields can be calculated from those containing primary fields only, by acting on them with a differential operator obtained from the transformation property (3.144). From global conformal invariance, we also know the two-point correlation functions of the primary fields, Eq. (3.139), and can construct the three-point function according to the same scheme. What about the $n$-point function for primary fields? It may happen that on a given level of the theory, say $k$, the states are not linearly independent but that there is a combination of states that vanishes (the family is then said to be degenerate at level $k$ ). The equation describing this degeneracy can then be transformed into a differential equation to be satisfied for an arbitrary correlation function of primary fields, if at least one of them is degenerate. In this way, it is possible to obtain, for conformal field theories with degenerate families, all correlation functions.

Unitary representations of the Virasoro algebra only exist for certain values of $c$ and $h$

$$
\begin{aligned}
& \qquad \begin{aligned}
& c \geq 1 \quad, \quad h \geq 0 \\
& \text { or } c=1-\frac{6}{m(m+1)}, \quad h_{p, q}(m)=\frac{[(m+1) p-m q]^{2}-1}{4 m(m+1)} \\
& \text { with } m=3,4, \ldots, \quad 1 \leq p \leq m-1 \quad, \quad 1 \leq q \leq p,
\end{aligned}
\end{aligned}
$$

and at least the discrete series (3.152) does indeed have degenerate families. The models belonging to this discrete series have quantized critical exponents [72] contained in (3.152). The most famous among them is the $2 \mathrm{D}$ Ising model with $c=1 / 2$.

Up to now, we have implicitly assumed that our fields are defined in the infinite $z$ plane. What happens when we consider finite systems? From Eq. (3.146), we deduce that

$$
\langle T(z)\rangle=\sum_{m=-\infty}^{\infty}\left\langle 0\left|\frac{L_{m}}{z^{m+2}}\right| 0\right\rangle=0
$$

in the infinite complex $z$ plane. Now use the exponential transformation

$$
z=\exp \left(\frac{2 \pi i}{L} u\right), \quad u=\frac{L}{2 \pi i} \log z
$$

to map the infinite $z$-plane onto a strip $(u)$ of width $L$ with periodic boundary conditions. Observe that under this transformation, the mode expansion (3.142) simply becomes a Fourier transformation, and the Virasoro generators $L_{n}$ become the Fourier coefficients of the stress-energy tensor. We obtain

$$
T_{\text {strip }}(u)=\left(T_{\text {plane }}(z)-\frac{c}{12}\{u, z\}\right)\left(\frac{d z}{d u}\right)^{2}
$$

and with (3.153) therefore

$$
\left\langle T_{\text {strip }}(u)\right\rangle=\frac{c}{24}\left(\frac{2 \pi}{L}\right)^{2} .
$$

The stress-energy tensor measures the cost of energy [change in the action $\delta S=(-1 / 2 \pi) \times$ $\left.\int T_{i j} \partial_{i} \xi_{j} d^{2} r\right]$ of a change in metric. One can now calculate the change in energy associated 
with another (nonconformal) transformation, a horizontal dilatation of the $u$-strip $\left[u_{1}^{\prime}=\right.$ $\left.(1+\varepsilon) u_{1}, u_{2}^{\prime}=u_{2}\right]$ which changes the length of the system, and integrate to find

$$
E(L)-E(\infty)=\frac{c \pi}{6 L}
$$

where $E(L)$ is the energy per unit length [71]. This formula is extremely important because it allows us to determine the value of the central charge from calculations on finite systems! Moreover, it suggests an interpretation of the anomaly (3.148) as a Casimir effect, i.e. a shift in the energy due to the finite geometry of the system. The mathematical reason is that the local conformal transformations (with the exception of the global ones) are (i) usually not defined in all points of the complex plane and (ii) are not one-to-one mappings of the complex plane on itself.

The exponential transformation (3.154) is also important to obtain the scaling dimensions of primary fields from finite-size calculations [73]. The two-point correlation function of a primary operator $\phi(z, \bar{z})$ with conformal weights $h, \bar{h}$ transforms under a conformal transformation (3.154) into

$$
\left\langle\phi(u, \bar{u}) \phi\left(u^{\prime}, \bar{u}^{\prime}\right)\right\rangle=\frac{(\pi / L)^{2 \Delta}}{\left(\sinh \left[\pi\left(u-u^{\prime}\right) / L\right]\right)^{2 h}\left(\sinh \left[\pi\left(\bar{u}-\bar{u}^{\prime}\right) / L\right]\right)^{2 \bar{h}}} .
$$

[Notice in passing: correlation functions at finite temperature $1 / \beta$ must satisfy periodic boundary conditions in the Matsubara time $\tau=i t$. (3.158) then gives directly the finite temperature expressions for the correlation functions of the preceding section if we put $L=2 v \beta$, as suggested in Section 3.3.] Writing $u=u_{1}+i u_{2}$ and going on the physical surface $\bar{u}=u^{\star}$, this can be expanded as

$$
\begin{aligned}
\left\langle\phi(u, \bar{u}) \phi\left(u^{\prime}, \bar{u}^{\prime}\right)\right\rangle & =\left(\frac{2 \pi}{L}\right)^{2 \Delta} \sum_{N, \bar{N}=0}^{\infty} a_{N} a_{\bar{N}} \exp \left[-2 \pi(\Delta+N+\bar{N})\left(u_{1}-u_{1}^{\prime}\right) / L\right] \\
& \times \exp \left[2 \pi i(s+N-\bar{N})\left(u_{2}-u_{2}^{\prime}\right) / L\right]
\end{aligned}
$$

Here, $\Delta$ and $s$ are scaling dimension and spin of the operator, respectively. The correlation function can also be calculated using operators $\hat{\phi}\left(u_{2}\right)$ which act on the states $|n, k\rangle$ of a Hilbert space

$$
\left\langle\phi(u) \phi\left(u^{\prime}\right)\right\rangle=\sum_{n}\left\langle 0\left|\hat{\phi}\left(u_{2}\right)\right| n, k\right\rangle e^{-\left(E_{n}-E_{0}\right)\left(u_{1}-u_{1}^{\prime}\right)}\left\langle n, k\left|\hat{\phi}\left(u_{2}^{\prime}\right)\right| 0\right\rangle
$$

where the matrix elements depend on $u_{2}$ as $\exp \left(i k u_{2}\right)$ with momentum $k$. Comparing (3.159) with (3.160), we find that energies and momenta scale as

$$
\begin{aligned}
E_{n}^{(L)}(N, \bar{N}) & =E_{0}^{(L)}+2 \pi v(\Delta+N+\bar{N}) / L \\
k^{(L)}(N, \bar{N}) & =k^{(\infty)}+2 \pi(s+N-\bar{N}) / L .
\end{aligned}
$$

Here, the energies are taken on the system of length $L$, and $v$ is the velocity of the excitations. In (3.162), we have allowed for a finite momentum $k^{(\infty)}$ of the highest weight state in the conformal tower built by the primary field $\phi$, extrapolated to the infinite 
system. Eqs. (3.161), (3.162) show that on a finite strip, each primary operator generates the whole spectrum of scaling dimensions and momenta of the conformal tower, i.e. also those of the descendant operators at level $(N, \bar{N})$.

There are thus two ways to obtain the scaling dimensions and correlation functions of a conformal field theory; (i) one can construct the stress-energy tensor; from its transformation properties 3.147) or its OPE with itself (3.141), one can deduce $c$, and from its OPE with other fields one obtains their scaling dimensions (3.140). (ii) one can study the finite size scaling behaviour of the ground state and a set of excited states, and obtain the central charge from Eq. (3.157). Going back now from $z, \bar{z}=z^{\star}$ to $(x, t)$ and extrapolating $L \rightarrow \infty$, the correlation functions of a primary $(h, \bar{h})$ field $\phi(x t)$ are then given from (3.139)

$$
\langle\phi(x t) \phi(00)\rangle=\frac{e^{i k^{(\infty)} x} e^{i \bar{k}^{(\infty)} x}}{(x+i v t)^{2 h}(x-i v t)^{2 \bar{h}}}
$$

and those of the descendant fields have the same structure with the corresponding $(h+$ $N, \bar{h}+\bar{N})$.

\subsubsection{The Gaussian model}

The first problem beyond the discrete classification scheme (3.152 - also the most important in the context of the present article - is given by theories with central charge $c=1$ and realized by free bosons, precisely the spinless Luttinger model, or the Gaussian model of statistical mechanics. Here, we give a conformal field theory analysis. The action for free bosons is given by

$$
S=\frac{1}{2 \pi} \int d z d \bar{z}\left(\partial_{z} \Phi\right)\left(\partial_{\bar{z}} \Phi\right) \quad, \quad \Phi \equiv \Phi(z, \bar{z})
$$

and the equivalence to the Luttinger model is clear when represented in terms of phase fields (3.49) or (3.58). The model is critical and manifestly conformally invariant, and remains so even upon introducing a dimensionless coupling constant $g$ as a prefactor in $S$, for all values of $g$ [cf. below after Eq. (3.187)]. The solution of the equations of motion can be given in terms of left- and right-moving (holomorphic and anti-holomorphic) fields $\Phi(z, \bar{z})=[\phi(z)+\bar{\phi}(\bar{z})] / 2$ where $z, \bar{z}=x \pm i y$. Their correlation functions are

$$
\langle\phi(z) \phi(w)\rangle=-\log (z-w) \quad, \quad\langle\bar{\phi}(\bar{z}) \bar{\phi}(\bar{w})\rangle=-\log (\bar{z}-\bar{w}) \quad .
$$

The fields $\phi(z)$ are not conformal fields, but their derivatives are. To show this, we need the stress-energy tensor which can be identified from the change in the action $\delta S=$ $(-1 / 2 \pi) \int T_{i j} \partial_{i} \xi_{j} d^{2} r$ under a conformal transformation $\mathbf{r} \rightarrow \mathbf{r}+\xi$ of the fields $\phi$ as (after going to the complex $z$-plane)

$$
T(z)=-\frac{1}{2}:[\partial \phi(z)]^{2}: \equiv-\frac{1}{2} \lim _{d \rightarrow 0}\left[\partial \phi\left(z+\frac{d}{2}\right) \partial \phi\left(z-\frac{d}{2}\right)-\frac{1}{d^{2}}\right],
$$

where the identity defines the normal-ordering convention. From the OPE of $\phi$ with the stress-energy tensor, we find

$$
T(z) \partial \phi(w)=\frac{\partial \phi(w)}{(z-w)^{2}}+\frac{1}{z-w} \partial^{2} \phi(w)+\ldots
$$


which, comparing with Eq. (3.140), indeed identifies $\partial \phi$ as a primary field with conformal weights $(1,0)$. It is now possible to write down a mode expansion (Laurent series) for the field $\partial \phi(z)$

$$
J(z) \equiv i \partial \phi(z)=\sum_{n=-\infty}^{\infty} \frac{J_{n}}{z^{n+1}}, \quad J_{n}=\oint \frac{d z}{2 \pi i} z^{n} J(z)
$$

Again, on a cylinder, i.e. periodic boundary conditions for a $(1+1)$ D field theory, the $J_{n}$ simply are the Fourier components of the current $J(z)$. Their algebra from (3.168) is

$$
\left[J_{n}, J_{m}\right]=n \delta_{n+m, 0}
$$

the $U(1)$-Kac-Moody algebra. We immediately note that the algebra of the $J_{n}$, (i) up to the factor $n$ which can be absorbed into a redefinition of $J_{n}$, is a bosonic one, and (ii) is identical to the algebra satisfied by the Luttinger density operators $\rho_{r s}$, Eq. (3.17). Physically, this identifies the $J_{n}$ as (chiral) density fluctuation modes or currents, which is the same because we look at a single branch only. The modes with $n<0$ are creation operators, and those with $n>0$ are annihilation operators

$$
J_{n}|0\rangle=0 \quad \text { for } \quad n>0
$$

The correlation function of the currents $J(z)=i \partial \phi(z)$ is

$$
\langle J(z) J(w)\rangle=\frac{1}{(z-w)^{2}}
$$

from (3.139) and the conformal weights $(1,0)$. Of course, for free bosons, these results can also be obtained directly by simply calculating a Gaussian integral.

Eq. (3.169) is a special case of a more general expression

$$
\left[J_{n}^{a}, J_{m}^{b}\right]=i f^{a b c} J_{n+m}^{c}+k n \delta^{a b} \delta_{n+m, 0}
$$

satisfied by the current generators $J_{n}^{a}$ of a more general Lie group, e.g. $S U(2)$ as appears in nonabelian bosonization schemes [20]. $f^{a b c}$ are its structure constants, and the integer $k$ is the level of the Kac-Moody algebra. The central charge of the associated Virasoro algebra is related to the level $k$ by $c=3 k /(k+2)$. We do not go into further details here, although we shall encounter an example of a $U(1)$-Kac-Moody algebra with $c \neq 1$ in Section 6.3 when we discuss the chiral Luttinger liquids formed by the edge excitations in the fractional quantum Hall effect.

One can also consider "vertex operators" : $\exp [i \alpha \phi(z)]$ : which, from their OPE with $T(z)$, are identified as primary fields with weights $\left(\alpha^{2} / 2,0\right)$. This determines the decay of their correlation functions, which, like all Gaussian model correlations, can also be evaluated explicitly

$$
\left\langle: e^{i \alpha \phi(z)}:: e^{-i \alpha \phi(w)}:\right\rangle=e^{\alpha^{2}\langle\phi(z) \phi(w)\rangle}=\frac{1}{(z-w)^{\alpha^{2}}} .
$$

The first equality is (3.75) and the second equality has been obtained with (3.165). 
Up to now, we have been silent on a parameter of the theory - the compactification radius $R$. This is a parameter of the theory, and one can either fix it from certain constraints on the vertex operators, or give it from the outset and then determine the operators which are well-behaved. Single-valuedness of the vertex operators implies that the fields $\phi(z)$ must be compactified with a compactification radius $R$ (obey periodic boundary conditions on a circle with radius $R$ )

$$
\phi+2 \pi R=\phi \quad, \quad R=n / \alpha
$$

In general, the vertex operators : $\exp [i \alpha \phi(z)]$ : have weird commutation relations. We can require, however, the object

$$
\Psi_{\alpha}^{\dagger}(z)=: \exp [i \alpha \phi(x)]:
$$

to obey fermionic anticommutation relations with $\Psi(z)$ and with its antiholomorphic counterparts $\bar{\Psi}^{\dagger}(\bar{z}), \bar{\Psi}(\bar{z})$, and to commute with the currents 40

$$
\begin{aligned}
\left\{\Psi(z), \Psi^{\dagger}\left(z^{\prime}\right)\right\} & =\delta\left(z-z^{\prime}\right) \quad, \quad\left\{\Psi(z), \Psi\left(z^{\prime}\right)\right\}=0 \\
\left\{\Psi(z), \bar{\Psi}^{\dagger}\left(\bar{z}^{\prime}\right)\right\} & =\left\{\Psi(z), \bar{\Psi}\left(\bar{z}^{\prime}\right)\right\}=0 \\
{\left[J(z), \Psi\left(z^{\prime}\right)\right] } & =-\delta\left(z-z^{\prime}\right) \Psi(z) .
\end{aligned}
$$

This imposes $\alpha=1$ for free fermions. : $\exp [i \phi(z)]$ : then creates a $(1,0)$ state from the vacuum. One can also prove that the current $J_{f}(z)=: \Psi^{\dagger}(z) \Psi(z)$ : written as a fermion bilinear is identical to the bosonic current $J_{b}(z)=i \partial \phi(z)$ where the field $\phi$ is precisely the one appearing in the exponential in (3.175), making the identity of the fermion and boson representations complete.

The Kac-Moody generators $J_{m}$ are extremely useful in classifying the excitations in our model. The Hamiltonian is related to the stress-energy tensor through $[x$ is the spatial coordinate of the image of $z$ on the cylinder]

$$
H=\frac{1}{2 \pi} \int[T(x)+\bar{T}(x)] d x \quad, \quad T(x)=\frac{1}{2}: J(x) J(x):+\frac{1}{24}\left(\frac{2 \pi}{L}\right)^{2} .
$$

The constant shows that our model has a central charge $c=1$. Putting together the mode expansions (3.142), (3.168) and the exponential transform (3.154), and realizing that the transformation to the cylinder generates an anomaly similar to (3.147) in : $J(z) J(z)$ :, we find the generators of the Virasoro algebra

$$
\frac{2 \pi}{L} L_{m}=\frac{1}{2 \pi} \int_{0}^{\infty} d x e^{i \frac{2 \pi}{L} m x} T(x)=\frac{\pi}{L} \sum_{n=-\infty}^{\infty}: J_{n} J_{m-n}:-\delta_{m, 0} \frac{c \pi}{12 L} .
$$

The $L_{m}$ satisfy the Virasoro algebra (3.143) with central charge $c=1$ among them, and

$$
\left[L_{n}, J_{m}\right]=-m J_{n+m}
$$

with the generators of the Kac-Moody algebra. To every Kac-Moody algebra, there is an associated Virasoro algebra, and the present construction of the Virasoro generators from 
the Kac-Moody ones is due to Sugawara. Specializing (3.179) to $n=0$ yields (taking only one chiral component)

$$
\left[H, J_{m}\right]=-\frac{2 \pi}{L} m J_{m}
$$

showing that the $J_{m}$ act as raising $(m<0)$ and lowering $(m>0)$ operators of a harmonic spectrum. The spectrum is harmonic because of the linearized dispersion and the equal $k$-spacing. Moreover, Eq. (3.170) implies that the state $|0\rangle$ is annihilated by the Virasoro generators (3.178) with $n>0$, and therefore qualifies as a highest-weight state.

The $J_{m}$ can be used to algebraically generate this spectrum and its conformal tower (family) of descendant states from a reference state $|0\rangle$. We suppose this state completely filled up to some energy, call it Fermi energy, $E_{F}=0$. By applying the $J_{m}$ with $m<0$, we make a particle-hole excitation with energy $-m$ by raising a particle from below the Fermi energy into an unoccupied state above, $-m$ levels higher. The energy level structure of the Hamiltonian carries over to the descendants created from the reference state $|0\rangle$

$$
J_{-n_{1}} \ldots J_{-n_{k}}|0\rangle \neq 0 \quad, \quad 1 \leq n_{1} \leq \ldots \leq n_{k}
$$

as in Eq. (3.150) for the Virasoro generators. The level of the state 3.181 ) is $\sum_{i=1}^{k} n_{i}$, $\left(n_{i}>0\right)$, and the level associated with a single generator $J_{-m}$ is $m(>0)$. The energy of the state equals its level in units of $2 \pi / L$. Of course, such a description is redundant: a state at level $N$ can be generated in $P(N)$ ways; $P(N)$ is the number of partitions of $N$. At level $N$, we have an N-particle-N-hole excitation. The Kac-Moody algebra can therefore be used to classify the particle-hole excitations from a reference state.

There are states which the currents cannot create from our Fermi sea $|0\rangle$ : those with additional particles, i.e. a total charge $Q$ with respect to $|0\rangle$, and specifically the lowestenergy states $|Q\rangle$ where the $Q$ particles occupy the first $Q$ states above the Fermi level. We anticipate that there is an infinity of such states, and each of them will be the highest weight state in the sector with total charge $Q$ of the theory. From our discussion, it is clear that the vertex operators (with $\alpha=1$ for a free theory)

$$
\Psi^{\dagger}(z)=: \exp [i \phi(z)]:
$$

creates a chiral fermion. The chiral state $|Q\rangle$ then is created out of $|0\rangle$ by

$$
|Q\rangle=: \exp [i Q \phi(z)]:|0\rangle
$$

The energy of a state $|Q\rangle$ is

$$
E(Q)-E(0)=v_{F} \frac{\pi Q^{2}}{L}
$$

Acting on $|Q\rangle$, the Kac-Moody generators $J_{-m}$ will again create the full spectrum of particle-hole excitations in the sector $|Q\rangle$.

There are more general operators than (3.182). One can build them by combining fields of both chiralities, writing e.g.

$$
\Psi_{m n}^{\dagger}(z, \bar{z})=: \exp \left(i\left[\alpha_{m n} \phi(z)+\bar{\alpha}_{m n} \bar{\phi}(\bar{z})\right]\right):
$$


Its scaling dimension is $\left(\alpha_{m n}^{2}+\bar{\alpha}_{m n}^{2}\right) / 2$, and $\alpha$ and $\bar{\alpha}$ are related to the compactification radius $R$ by

$$
\alpha_{m n}=\frac{1}{2}\left(\frac{m}{R}+\frac{n R}{2}\right) \quad, \quad \bar{\alpha}_{m n}=\frac{1}{2}\left(\frac{m}{R}-\frac{n R}{2}\right) .
$$

Its physical meaning is quite clear. Increasing $m$ means increasing the charge for both chiralities $\Psi_{m 0}^{\dagger}|Q, \bar{Q}\rangle=|Q+m, \bar{Q}+m\rangle$. Increasing $n$ transfers charge from one chirality to the other, i.e. creates a finite persistent current $\Psi_{0 n}^{\dagger}|Q, \bar{Q}\rangle=|Q+n, \bar{Q}-n\rangle$ [this is an example of a primary operator with $k^{(\infty)} \neq 0$ in (3.162)]. $\Psi_{m n}^{\dagger}$ creates charge or current excitations, or combinations thereof. As for $\Psi_{\alpha}(z)$, the particles making up these charge and current excitations are fermions only at the particular compactification radius $R=1$. Else, they are more general objects.

The Luttinger or Gaussian model has operators with continuously varying exponents. Responsible for this are dimension $(1,1)$ marginal operators which take the model along a whole critical line. The simplest of these operators is

$$
S^{\prime}=\frac{g-1}{2 \pi} \int d z d \bar{z}\left(\partial_{z} \Phi\right)\left(\partial_{\bar{z}} \Phi\right)
$$

which is proportional to the free action (3.164) and whose only effect is to introduce the coupling constant $g$ as a prefactor into $S$. The effect of this interaction can simply be absorbed by redefining the fields $\phi \rightarrow \phi / \sqrt{g}$. With this redefinition, the effective $\alpha \rightarrow \alpha / \sqrt{g}$ in the vertex operators changes accordingly, and consequently both their compactification radius and their conformal weight. When the compactification radius $R \neq 1, \Psi_{\alpha}^{\dagger}$ no longer describes a fermion but a more complicated object. In the Luttinger model, the $g_{2}$-interaction is such a marginal operator, coupling currents of both chiralities. From Eq. (3.173) it is obvious then that $S^{\prime}$ generates continuously varying exponents in the correlation functions, and varying $g$ sweeps the model over a whole critical line.

By transforming the phase-field representation of the spinless Luttinger model 3.58 into an imaginary time $(\tau=i t)$ action, one obtains the Gaussian model with an effective coupling constant $g=K$. Importantly, the coupling constant $g$ depends essentially on $g_{2}$. Finite $g_{4}$ only renormalizes the effective $g_{2}$ but, alone, is not able to give $g \neq 1$. This is quite easy to understand because it only changes the Fermi velocity of the Luttinger model and is therefore absorbed when going to the second spatial coordinate $y=v \tau$.

More interesting is the case when the interactions are not of current-current type, or when the theory is formulated on a lattice so that the identification of the conformal operators is far from obvious. Mironov and Zabrodin have given a simple application of the methods discussed above to interacting spinless fermions (or bosons) [74]

$$
H=\int_{0}^{L} d x \partial_{x} \psi^{\dagger}(x) \partial_{x} \psi(x)+\frac{g}{2} \int_{0}^{L} d x d y \psi^{\dagger}(x) \psi^{\dagger}(y) V(x-y) \psi(y) \phi(x)
$$

$V(x)$ is a repulsive pair interaction of rather general form. The density of particles is $n=N / L$ and $k_{F}=\pi n$. The model can be solved by Bethe Ansatz for $V(x)=\delta(x)$ but most of the results are expected to carry over for reasonable longer-range potentials.

From the finite-size scaling formula for the ground state energy (3.157), one finds $c=1$ which puts it in the Gaussian (Luttinger liquid) universality class. Now we want 
to find the correlation function of some local operator $O(x)$. To this end, one must compute up to order $1 / L$ the energy of the lowest excited state $|\varphi\rangle$ whose matrix element $\langle 0|O(x)| \varphi\rangle \neq 0$ for $L \rightarrow \infty$. Then, one can use Eq. (3.163) if its scaling dimension and spin is known from (3.161) and (3.162). As an example, one can make particlehole excitations $[\rho(x)]$ with momentum $2 m \pi / L(m$ small). From their excitation energies linear in $m$, one can deduce the renormalized sound velocity $v$ and $\Delta= \pm s=1$. Of course, this is in agreement with our earlier discussion where we found that the currents of the Gaussian model are $(1,0)$ or $(0,1)$ fields. Next, make an excitation at constant particle number with $k_{0 n}^{(\infty)}=2 n k_{F}$ where the particle-hole spectrum goes to zero. In a free system, this would correspond to applying the operator $\Psi_{0 n}^{\dagger}$ to the Fermi sea. The Bethe Ansatz gives the energy change $\delta E_{0 n}=(2 \pi / L)\left(2 k_{F} n^{2} / v\right)$ and, using (3.161), the scaling dimension $\Delta_{0 n}=2 k_{F} n^{2} / v$. Comparing with (3.186), one finds a compactification radius $R^{2}=2 k_{F} / v$. One can also add pairs of particles or a single particle, where a selection rule enforces half-integer $n$. The energy shift is given by (3.161) with a scaling dimension (3.186) with $m=1, n=1 / 2$ and the $R$ found above. The Green function and the CDW correlation functions (corresponding to the low-energy excitation at $2 k_{F}$ discussed before) are then

$$
G(x) \sim \cos \left(k_{F} x\right) x^{-1 / 2 R^{2}-R^{2} / 2}, \quad R_{C D W}(x) \sim \cos \left(2 k_{F} x\right) x^{-2 R^{2}}, \quad R^{2}=2 k_{F} / v=K .
$$

The exponents satisfy the scaling relations of the spinless Luttinger model, and the correlation exponent $K$ has been identified in the last equality. (The factor 4 difference in $R^{2}$ to Mironov and Zabrodin [74 must be due to a different prefactor $[2 / \pi]$ of the Gaussian action.)

The close correspondence of conformal field theory and the operator approach to bosonization should be apparent now, at least for the case $c=1$ of the Luttinger and Gaussian models. Density fluctuations (currents), charge and current excitations, and fermion raising and lowering operators all appear either in an operator approach based on a Hilbert space, or in an algebraic formalism based on the $U(1)$-Kac-Moody algebra, which is satisfied by the currents as a consequence of the $U(1)$-gauge symmetry (3.9) corresponding to the conservation of left- and right-moving particles separately. The main problem in the operator approach is the explicit identification of the coupling constant of the Luttinger model which then determines all correlation functions. In conformal field theory, we must determine the scaling dimensions of the various primary operators. As will be discussed in the next chapter, in both cases the spectrum of low-lying eigenvalues is sufficient for that purpose, showing once more the full equivalence between bosonization and conformal field theory. Which one to use is a matter of taste.

The preceding analysis of the interacting spinless fermions foreshadows the application of conformal field theory to rather general models of interacting electrons. We shall discuss this topic in the next chapter, where some other methods for extracting the low-energy physics will also be presented. 


\section{Chapter 4}

\section{The Luttinger Liquid}

\subsection{The conjecture}

The Luttinger model can be solved exactly at any interaction strength, except for too strong attraction where the model becomes unstable towards phase separation (formation of electron droplets; Section 5.3). However, it contains drastic approximations with respect to a realistic many-body problem: (i) its dispersion is strictly linear, and (ii) the electron-electron interaction is limited to forward scattering only. The possibility of an exact solution is precisely related to these two approximations.

One may therefore wonder if these approximations are essential in the sense that the Luttinger physics is lost in any different model or if, on the contrary, this physics is robust. In this case, only parameters $\left(K_{\nu}, v_{\nu}\right)$ would be renormalized close to the Fermi surface, but the structure of the low-energy theory would be identical to the Luttinger model. Of course, further away from the Fermi surface, new phenomena such as boson-boson interactions or lifetime effects could occur but it would be guaranteed that they fade away as $\left(k-k_{F}, \omega, T\right) \rightarrow 0$. This is what happens in the Fermi liquid. The Luttinger model would then represent the generic behaviour of gapless 1D quantum systems, and one could build upon it the universal low-energy phenomenology for all 1D metals (the Luttinger liquid) called for by the breakdown of the Fermi liquid in 1D (Chapter 2).

Haldane, in the early 1980's, conjectured that this was indeed possible and supported this conjecture with a series of case studies of models solvable by Bethe Ansatz [49, 75]. He also demonstrated that certain features mapped away when passing to the Luttinger model, such as curvature in the dispersion, only introduce nonsingular, perturbative interactions among the bosons [3] which disappear as one goes to the long-wavelength or low-frequency limit. Haldane's conjecture has meanwhile been verified in an impressive number of instances some of which will be discussed below and, to my knowledge, no counterexample has been discovered yet.

What is the content of this "Luttinger liquid conjecture"? Given any 1D model of correlated quantum particles (in 1D not even necessarily fermions) and let there be a branch of gapless excitations: then the Luttinger model is the stable low-energy fixed point of the original model (or at least its gapless degrees of freedom). In other words, the asymptotic 
low-energy properties of the degree of freedom associated with this branch are described by an effective (renormalized) Luttinger model, in particular with one renormalized Fermi velocity $v_{\nu}$ and one renormalized effective coupling (stiffness) constant $K_{\nu}$, up to perturbative boson-boson interactions. All properties found for the Luttinger model: (i) absence of fermionic quasi-particles in the vicinity of the Fermi surface, (ii) anomalous dimensions of the fermion operators producing nonuniversal power-law correlations, (iii) charge-spin separation, (iv) the universal relations among the nonuniversal exponents of the correlation functions, among the velocities for sound, charge, and current excitations and between velocities and the effective renormalized coupling constants, carry over to the low-energy sector of the model under consideration. The nontrivial task remaining then is to determine the two central quantities of the Luttinger liquid, the renormalized velocity $v_{\nu}$ and the renormalized effective coupling constant $K_{\nu}$, from the original model. Once this is achieved, one has an asymptotically exact solution of the $1 \mathrm{D}$ many-body problem. This is what most of this chapter will be about.

A word of caution is required for systems with several degrees of freedom. If all degrees of freedom remain gapless, the low-energy fixed point will be a Luttinger liquid in the sense described. If some degrees of freedom become gapped while others do not, the physics within the gapless degrees of freedom can be described as a Luttinger liquid while all quantities involving gapped degrees of freedom will deviate qualitatively from the Luttinger liquid. In Chapter 5, we will give examples for this kind of situation.

When mapping a more realistic model of interacting electrons in 1D onto the Luttinger model, complications arise from two sources: (i) the dispersion of these models is not linear; (ii) the interactions generally do not only contain the forward scattering processes included in, and solved by the Luttinger model but also the large momentum transfer backward (spin exchange) and Umklapp scattering (for commensurate band filling) depicted in Fig. 3.3. Moreover if the interactions are not weak, states far from the Fermi surface are coupled to the Fermi surface states; curvature and interaction could then conspire to invalidate the Luttinger liquid picture. That none of this in fact happens, was demonstrated by Haldane [3, 49].

There are two basic ways of proceeding. One can start from a Luttinger model and extend it by various interactions and other features, and then study the stability and renormalization of the Luttinger model solution. The alternative is to start directly from a realistic model of $1 \mathrm{D}$ correlated fermions, possibly on a lattice, and search for either Luttinger liquid-correlations or the specific Luttinger liquid properties of the spectrum of charge, current and sound excitations, Eq. (3.32). The organization of the chapter will follow roughly this order. 


\subsection{Luttinger model with nonlinear dispersion - the emergence of higher harmonics}

Haldane extended the Luttinger Hamiltonian, Eqs. (3.1)-(3.4), by terms modelling nonlinear dispersion

$$
\begin{aligned}
\varepsilon_{r}(k) & =v_{F}\left(r k-k_{F}\right) \\
& \rightarrow v_{F}\left(r k-k_{F}\right)+\frac{1}{2 m}\left(r k-k_{F}\right)^{2}+\frac{\lambda}{12 m^{2} v_{F}}\left(r k-k_{F}\right)^{3}
\end{aligned}
$$

The third order term is necessary to ensure stability, and $\lambda>3 / 4$ is required then. (4.1) can be bosonized, and one obtains quadratic terms of the usual structure (3.21), with parameters renormalized by $m$ and $\lambda$, but also cubic and quartic boson terms. The quadratic form can be diagonalized as usual and the remaining terms are written as (for spinless fermions for simplicity [3])

$$
\delta H=\sum_{r} \frac{1}{2 \pi} \int_{0}^{L} d x: \frac{1}{6 m} \tilde{\Phi}_{r}^{3}(x)+\frac{\lambda}{48 m^{2} v_{F}} \tilde{\Phi}_{r}^{4}(x):
$$

where the fields $\Phi_{r}(x)=\left[r \Phi_{\rho}(x)-\Theta_{\rho}(x)\right] / \sqrt{2}$ are given in terms of the phase fields of Eqs. (3.42) and (3.43) and the tilde implies that the Bogoliubov transformed fields (3.50) enter. $\delta H$ describes boson-boson interactions. Their appearance is quite clear physically because, for a curved dispersion, two fluctuations with wave vector $q$ and $q^{\prime}$ will have an energy different from a single one with $q+q^{\prime}$ : fluctuations interact. Fortunately, $\delta H$ is harmless: one can either (i) argue, using renormalization group, that these higher order boson terms are irrelevant and therefore do not influence the fixed point physics described by the quadratic ones, or (ii) as did Haldane, perform a systematic $1 / m$-expansion to find that the model still obeys the constitutive Luttinger liquid relations (3.32) between velocities and coupling constant, and that the relation of $K$ to the correlation function exponents, Eqs. (3.85) - (3.100), also remains unchanged. Only the values of $K$ and the velocities change.

There is, however, another important effect caused by the nonlinear dispersion: the appearance, in the physical fermion operators $\Psi(x)$, of higher harmonics in the chiral fermion operators $\Psi_{r}(x)$ which generates components with $3 k_{F}, 5 k_{F}, \ldots$ in the fermion fields and with $4 k_{F}, 6 k_{F}, \ldots$ in the pair fields. Consequently, the single-particle Green function acquires components at $3 k_{F}, 5 k_{F} \ldots$, and e.g. the density-density correlations get oscillations at $4 k_{F}, 6 k_{F} \ldots$ in addition to the usual ones at small $q$ and at $2 k_{F}$. These components are not present in the Luttinger model but may appear in any more general model with nonlinear dispersion $\varepsilon(k)$. They are necessary for constructing local density and fermion operators.

This becomes apparent when one attempts to construct a representation for excitations on all length scales from the long-wavelength ones which dominate the low-energy physics [41]. Recall from our bosonization procedure in Section 3.2.2 that the field $\Phi(x)$ had a kink of amplitude $\pi$ at the location of each particle. The location of the $l$ th particle then 
is given by $\Phi(x)=l \pi$. When going from the smeared, long-wavelength density operators $\rho_{r}(x)$ to a physical local operator $\rho(x)$, it is sufficient to multiply by a delta function $\sum_{l} \delta[\Phi(x)-l \pi]$ to locate the individual particles. The local density then is written as

$$
\rho(x)=[n+\Xi(x)] \sum_{m=-\infty}^{\infty} \exp \left[2 i m\left\{\Phi(x)+k_{F} x\right\}\right]
$$

where $n=N / L$ is the average density, and the field $\Xi(x)$ describes the long-wavelength fluctuations. The fermion field essentially is the square-root

$$
\Psi(x) \sim \sqrt{n+\Xi(x)} \exp [i \Theta(x)] \sum_{m=-\infty}^{\infty} \exp \left[(2 m+1) i\left\{\Phi(x)+k_{F} x\right\}\right],
$$

but the sum must contain only odd terms to ensure the anticommutation property. $\Theta(x)$ and $\Phi(x)$ have been introduced in (3.42) and (3.43), and $\Xi(x)$ commutes with $\Theta(x)$ as $\left[\Theta(x), \Xi\left(x^{\prime}\right)\right]=i \delta\left(x-x^{\prime}\right)$. Moreover, describing charge density fluctuations, $\Xi(x)$ is related to $\Phi(x)$ by $\partial_{x} \Phi(x)=-\pi[n+\Xi(x)]$. An equation equivalent to (4.4) for spin$1 / 2$ fermions can be constructed easily. The correlation exponents associated with these higher harmonics are then deduced with the methods of Section 3.3 [3].

In the Luttinger model with its linear dispersion, only the components with $m=0, \pm 1$ are present in (4.3) and those with $m=-1,0$ in (4.4). This is related to the fact that in the Luttinger model, the mean current is [from the continuity equation (3.106) for $q \rightarrow 0] j=v_{j} J / L$, and is strictly conserved because $J$ is a good quantum number [3]. With $\delta H$ [Eq. (4.2)] containing the nonlinearity, the current operator as determined from the continuity equation contains higher-order boson terms, and a simple relation to the quantum number $J$ only obtains close to the Fermi surface. In order to allow for a fermionic representation of this complex current operator, the physical fermions must contain the higher harmonics in the chiral fermions.

\subsection{Backward and Umklapp scattering}

We now turn to the problem of non-Luttinger interactions. The Luttinger model includes only the forward scattering interactions $g_{2}$ and $g_{4}$, Eqs. (3.3) and (3.4). This is certainly very restrictive since any realistic model, say with an interaction

$$
H_{i n t}=\frac{1}{L} \sum_{k, k^{\prime}, q, s, s^{\prime}} V(q) c_{k+q, s}^{\dagger} c_{k^{\prime}-q, s^{\prime}}^{\dagger} c_{k^{\prime}, s^{\prime}} c_{k, s}
$$

will also contain components of $V(q)$ with $q$ large, specifically $q \approx 2 k_{F}$. The restriction to forward scattering is, however, absolutely essential in guaranteeing the exact solvability of the Luttinger model.

In any realistic theory, these offending interactions will be there. The most important processes are depicted in Figure 3.3. The contributions to the Hamiltonian are

$$
H_{1 \perp}=g_{1 \perp} \sum_{s} \int_{0}^{L} d x: \Psi_{+, s}^{\dagger}(x) \Psi_{-, s}(x) \Psi_{-,-s}^{\dagger}(x) \Psi_{+,-s}(x):
$$




$$
\begin{aligned}
& =\frac{2 g_{1 \perp}}{(2 \pi \alpha)^{2}} \int d x \cos \left[\sqrt{8} \Phi_{\sigma}(x)\right], \\
H_{3 \perp} & =\frac{g_{3 \perp}}{2} \sum_{s} \int_{0}^{L} d x: \Psi_{+, s}^{\dagger}(x) \Psi_{+,-s}^{\dagger}(x) \Psi_{-,-s}(x) \Psi_{-, s}(x)+\text { H.c. : } \\
& =\frac{2 g_{3 \perp}}{(2 \pi \alpha)^{2}} \int d x \cos \left[\sqrt{8} \Phi_{\rho}(x)\right] .
\end{aligned}
$$

The first term represents exchange scattering of two counterpropagating particles with opposite spin across the Fermi surface (momentum transfer $\approx 2 k_{F}$ ) and violates spincurrent conservation. [SU(2)-invariant backscattering would imply the presence of a $g_{1 \|^{-}}$ term in the Hamiltonian which, after bosonization, can however be absorbed into $g_{2 \nu} \rightarrow$ $g_{2 \nu}-g_{1 \|} / 2$.] The second term is Umklapp scattering of two particles moving in the same direction. The product of four fermion fields in Eq. (4.8) contains a factor $\exp \left(4 i k_{F} x\right)$ which, generally, oscillates rapidly and suppresses contributions from this term. For half-filled bands, however, $4 k_{F}$ equals a reciprocal lattice vector $\pm 2 \pi / a$, and Umklapp scattering becomes important. Charge-spin separation is respected here. This is not generic. However all purely electronic processes coupling charge and spin, i.e. arising from four fermion operators, are less relevant than (4.6) and (4.8) [50].

For the Luttinger liquid phenomenology to survive one must demonstrate that, although these interactions certainly renormalize velocities and stiffness constant, they do not destroy the universal relations among them nor those between $K$ and the correlation function exponents. To map the low-energy physics onto a Luttinger model, one then has to (i) check that there are (how many?) branches with gapless excitations; (ii) for each branch determine the relevant renormalized velocity and coupling constant; (iii) insert these into the Luttinger liquid expressions for the quantities of interest. If this works, it is proven that the originally offending interactions are irrelevant at the Luttinger liquid fixed point and that their only effect was a quantitative renormalization of the Luttinger liquid parameters.

This is the spirit of all approaches to a Luttinger liquid description of interacting $1 \mathrm{D}$ electrons. It is most explicit in the renormalization group method if one accepts the limitation to weak coupling, and we shall treat $H_{1 \perp}$ in this way. While more powerful mappings of lattice models onto the Tomonaga-Luttinger model are available now, in conjunction with renormalization group, such "direct" extensions of the TomonagaLuttinger model give a clear and simple idea of how the renormalized effective parameters in the Luttinger liquid are generated. Moreover, many problems beyond the 1D electronelectron-interaction models, such as coupling to phonons or scattering by impurities, only become tractable with this approach. Also renormalization group allows to determine corrections to the simple power-law decay $(3.92)-(3.100)$ of correlation functions of the Luttinger model which are absolutely essential to obtain a correct picture of the physics of more complicated models. Finally, much of the early understanding of what is now called "Luttinger liquid" was based on continuum models 18, 19, by that time most often running under the label "g-ology", and renormalization group was the most important tool for their understanding.

We derive renormalization group equations for $H_{1 \perp}$ following a method described by 
Chui and Lee [76]. There are other ways to formulate the renormalization group; they have been reviewed in detail elsewhere [18, 19, 22. First diagonalize the Luttinger part of $H_{\sigma}$ (shorthand for all terms containing $\sigma$-operators). Then compute the partition function $Z_{\sigma}=\left\langle\exp -\beta H_{\sigma}\right\rangle$ in the Matsubara formalism of imaginary times $\tau=i t . Z_{\sigma}$ can be expanded in $H_{1 \perp}$ and the expectation value be evaluated with respect to the diagonal part

$$
Z_{\sigma}=\sum_{n} \frac{1}{(n !)^{2}}\left(\frac{g_{1 \perp}}{(2 \pi)^{2}}\right)^{2 n} \int\left(\prod_{i}^{2 n} \frac{d^{2} r}{\alpha^{2}}\right) \exp \left[2 K_{\sigma} \sum_{i>j} q_{i} q_{j} \ln \left(\frac{\left|\mathbf{r}_{i}-\mathbf{r}_{j}\right|^{2}}{\alpha^{2}}\right)\right]
$$

The $2 \mathrm{D}$ vector $\mathbf{r}=\left(x, v_{\sigma} \tau\right)$ and $q_{i}=1$ for $i=1 \ldots n$ and -1 else. $Z_{\sigma}$ is now identified as the partition function of a classical 2D Coulomb gas with charges $q_{i}$, at a fictitious temperature $\beta_{C G}=4 K_{\sigma}$ and a fugacity $g_{1 \perp} /(2 \pi)^{2}$. For this problem Kosterlitz and Thouless [77] derived a set of renormalization group equations which translate into

$$
\frac{d K_{\sigma}}{d \ell}=-\frac{1}{2} K_{\sigma}^{2}\left(\frac{g_{1 \perp}}{\pi v_{\sigma}}\right)^{2} \quad, \quad \frac{d g_{1 \perp}}{d \ell}=g_{1 \perp}\left(2-2 K_{\sigma}\right)
$$

They describe the flow of the effective coupling constants $g_{1 \perp}$ and $K_{\sigma}$, shown in Figure 4.1 , when short-distance degrees of freedom (between $\alpha$ and $\alpha e^{\ell}$ ) are integrated out. Here $\alpha$ is reinterpreted as a short-distance cutoff parameter which may be of the order of a lattice constant. The coupling constants must be rescaled so as to maintain the Fermi surface physics and the asymptotic correlations invariant. There are two different types of flow. (i) Assume $K_{\sigma}$ sufficiently large so that $\left|g_{1 \perp}\right|$ decreases with increasing $\ell$ (lower right part of Fig. 4.1). If this remains so even for $\ell \rightarrow \infty$, the renormalization group trajectory will flow into a fixed point $g_{1 \perp}^{\star}=0$ and $K_{\sigma} \rightarrow K_{\sigma}^{\star} \cdot g_{1 \perp}$ has dropped out of the problem, i.e. at long distances the model behaves effectively as a Luttinger model with a renormalized $K_{\sigma}^{\star}$. The fixed point is spin-rotation invariant if it turns out that $K_{\sigma}^{\star}=1$. Then the flow is precisely along the separatrix. This is one example of a Luttinger liquid. [Even then, during intermediate stages of the calculation, one may have $K_{\sigma}(\ell) \neq 1$; this apparent breaking and final restoration of $S U(2)$-invariance is typical of abelian bosonization.] (ii) If the bare $K_{\sigma}$ is not large enough compared to $\left|g_{1 \perp}\right|, K_{\sigma}$ will flow towards 0 but more importantly $\left|g_{1 \perp}\right|$ will increase. Derived from a perturbation expansion, the renormalization group manifestly looses its sense. It is clear that the system flows away from the Luttinger liquid fixed line, and the diverging $\left|g_{1 \perp}\right|$ signals an instability of the model towards a different ground state whose accurate description must, however, be based on different methods. This regime will be the subject of Section 5.1 .

So long as the system is not half-filled, the charge exponent $K_{\rho}$ is not renormalized. At half-filling, the situation in the charge degrees of freedom is isomorphic to the spin part discussed here. It is sufficient to change $g_{1 \perp} \rightarrow g_{3 \perp}, K_{\sigma} \rightarrow K_{\rho}$ and carry over the Equations (4.11). Also more complicated models where charge-spin coupling is important can be treated in this way [50]. The application to phonons and impurities will be discussed below. 
The essential weakness of the renormalization group approach is its limitation to weak coupling, being derived from perturbation expansions. This limitation has been overcome by several methods which will be discussed in the subsequent sections.

Before, however, we discuss in more details the correlation functions of such a Luttinger liquid where all non-Luttinger interactions have become irrelevant. A first idea about the correlations is obtained by inserting the fixed point value $K_{\nu}^{\star}$ into the correlation functions of Section 3.3. This is the standard procedure in the renormalization group treatment of critical points [70. In particular, we would then find a degeneracy of exponents between SDW and CDW, and SS and TS, no matter what the precise fixed point values $K_{\nu}^{\star}$. Anticipating that the non-half-filled repulsive Hubbard model can be described, at least for small $U$, by $(3.1)+(4.6)$, it is clear that this cannot be the whole story.

Let us consider the $2 k_{F^{-}} S D W_{z}$ correlation function for definiteness. The $S D W_{z^{-}}$ operator is

$$
O_{S D W_{z}}^{\dagger}(x)=\sum_{s} s \Psi_{-, s}^{\dagger}(x) \Psi_{+, s}(x)=\frac{-i}{\pi \alpha} \exp \left[2 i k_{F} x-\sqrt{2} i \Phi_{\rho}(x)\right] \sin \left[\sqrt{2} i \Phi_{\sigma}(x)\right] .
$$

Now consider the time-ordered correlation function (again in imaginary-time formalism)

$$
\begin{aligned}
-R_{S D W_{z}}(\mathbf{r}) & =\left\langle T_{\tau} O_{S D W_{z}}(\mathbf{r}) O_{S D W_{z}}^{\dagger}(\mathbf{0})\right\rangle \\
& =\frac{1}{Z_{\sigma}} \operatorname{Tr}\left(T_{\tau} O_{S D W_{z}}(\mathbf{r}) O_{S D W_{z}}^{\dagger}(\mathbf{0}) \exp \left[\int_{0}^{\beta \rightarrow \infty} d \tau H(\tau)\right]\right),
\end{aligned}
$$

where $H=H_{\text {Lutt }}+H_{1 \perp}$ and the trace (Tr) is performed over $\sigma$ and $\rho$. The charge part is trivial and gives the Luttinger result $|\mathbf{r}|^{-K_{\rho}}$. In the spin part, use Wick's theorem to expand the exponential in $H_{1 \perp}$. This will generate nonvanishing contributions at all even orders which are essentially those contained in the partition function $Z_{\sigma}$ multiplied by $O O^{\dagger}$. In addition to these terms there will, however, be important new terms in odd orders of $H_{1 \perp}$ not present in the partition sum [78, 79]. They arise from contracting the $\sigma$-part of the $S D W_{z}$-operators with $H_{1 \perp}$

$$
\begin{gathered}
\left\langle\sin \left[\sqrt{2 K_{\sigma}} \Phi_{\sigma}(\mathbf{r})\right] \sin \left[\sqrt{2 K_{\sigma}} \Phi_{\sigma}(\mathbf{0})\right] \cos \left[\sqrt{8 K_{\sigma}} \Phi_{\sigma}\left(\mathbf{r}_{1}\right)\right]\right\rangle \\
=-\frac{1}{8}\left\langle\exp \sqrt{2 K_{\sigma}}\left[\Phi_{\sigma}(\mathbf{r})+\Phi_{\sigma}(\mathbf{0})-2 \Phi_{\sigma}\left(\mathbf{r}_{1}\right)\right]+\text { H.c. }\right\rangle
\end{gathered} .
$$

These expectation values do not vanish because the prefactor of the $\Phi_{\sigma}$-field in the correlation function is half of that in the perturbation operator or, in other words, because the $\Psi_{-, s}^{\dagger} \Psi_{+, s}$-components of the $S D W_{z}$-operator also occur as factors in $H_{1 \perp}$. In the Coulomb gas language, this is equivalent to saying that one considers the screening of two test charges $q / 2$ by charges $-q$. The terms up to second order can be reexponentiated in the spirit of a cumulant expansion. Now it is important to integrate up the correlation function along the whole renormalization group trajectory [78, 79. The spin-part of the correlation function then becomes

$$
R_{S D W_{z}}^{(\sigma)}(\mathbf{r} ; \alpha)=\exp \left(-K_{\sigma} \ln \frac{|\mathbf{r}|}{\alpha}+\int_{0}^{\ell} \frac{g_{1 \perp}\left(\ell^{\prime}\right)}{\pi v_{\sigma}} d \ell^{\prime}+\frac{1}{2} \int_{0}^{\ell}\left[\frac{g_{1 \perp}\left(\ell^{\prime}\right)}{\pi v_{\sigma}}\right]^{2} \ln \frac{|\mathbf{r}|}{\alpha} d \ell^{\prime}\right) .
$$


If scaling goes to weak coupling, the integrals can be extended to infinity and the usual expressions involving the fixed-point exponent $K_{\sigma}^{\star}$ follow. Notice, however, that an ultimate cutoff is provided by the observation scale $|\mathbf{r}|$ (if not by temperature or system size) so that the integration cannot go beyond $\ell^{\star}=\ln |\mathbf{r}| / \alpha$. The correlation function then decays as

$$
R_{S D W_{z}}(\mathbf{r})=\left(\frac{|\mathbf{r}|}{\alpha}\right)^{-K_{\rho}-K_{\sigma}^{\star}} \sqrt{\ln \frac{|\mathbf{r}|}{\alpha}}, \quad K_{\sigma}^{\star}=1,
$$

where we have reintroduced the contribution from the charge density fluctuations. In doing the integrals, we have used explicitly the fact that we scale along critical line (the separatrix in Fig. 4.1) so that the logarithmic corrections only obtain in the spinrotation invariant case. In this case, one recovers expressions identical to (4.16) for the $x$ - and $y$-components of the $S D W$ correlation function although, involving $\Theta_{\sigma}$-fields, the intermediate expressions are quite different. The charge density wave and superconducting correlations decay as

$R_{C D W}(\mathbf{r}) \sim|\mathbf{r}|^{-K_{\rho}-1} \ln ^{-3 / 2}|\mathbf{r}|, \quad R_{S S}(\mathbf{r}) \sim|\mathbf{r}|^{-K_{\rho}^{-1}-1} \ln ^{-3 / 2}|\mathbf{r}|, \quad R_{T S}(\mathbf{r}) \sim|\mathbf{r}|^{-K_{\rho}^{-1}-1} \ln ^{1 / 2}|\mathbf{r}|$.

There is no logarithmic correction to the $4 k_{F-} \mathrm{CDW}$ function because it does not involve spin fluctuations. It is remarkable that at this level, the degeneracy of the CDW and SDW correlation functions and between SS and TS is lifted: they have the same exponents, correctly given by the Luttinger model but the correlations are logarithmically stronger for SDW and TS. For repulsive interactions, magnetic correlations must dominate! If we have attractive backscattering $g_{1 \perp} \rightarrow-g_{1 \perp}$ with $K_{\sigma}$ left unchanged, CDW and SS will be logarithmically enhanced over SDW and TS [just exchange the log-exponents in (4.16) and (4.17)]. Finally, if spin-rotation invariance is broken and there is an easy plane anisotropy, $g_{1 \perp}$ scales to zero faster. In this case, the integration along the trajectory only gives prefactor corrections to the power-law correlations [79]. These results can be transposed straightforwardly to commensurate systems when Umklapp scattering is irrelevant [50].

The phase diagram in the $g_{1 \perp}-K_{\rho}$-plane obtained in the absence of Umklapp scattering is displayed in Figure 4.2. At $g_{1 \perp}>0$, the dominant divergences are SDW for $K_{\rho}<1$ and TS for $K_{\rho}>1$. Subdominant fluctuations are indicated in parenthesis, and the preceding discussion shows that CDW and SS have the same exponents as SDW and TS but are disfavoured by their logarithmic corrections. We have assumed the system to be spinrotation invariant, and consequently, the fixed-point $K_{\sigma}^{\star}=1$. For $g_{1 \perp}<0$, a spin gap opens through a Kosterlitz-Thouless transition, and formally $K_{\sigma}^{\star}=0$. Here, CDW and SS have the strongest divergences for $K_{\rho}<1$ and $K_{\rho}>1$, respectively. They also diverge in the regimes $1 \leq K_{\rho} \leq 2$ and $1 / 2 \leq K_{\rho} \leq 1$, respectively, though with a weaker power than the dominant fluctuations.

Logarithmic corrections to the free energy of statistical models whose fermionic description contains a marginally irrelevant Umklapp operator and which are related to the singularities found here in the correlation functions, had been discovered earlier by Black and Emery 80 . 


\subsection{Lattice models: Hubbard \& Co.}

A variety of nontrivial lattice models can be solved exactly in $1 \mathrm{D}$, for which no exact solution exists in higher dimension. A non-exhaustive list contains the Heisenberg model [81], the Hubbard model [82, 83] and various long-range, supersymmetric or degenerate extensions, the supersymmetric $t-J$-model [84]-[86], and others. Solvable continuum models include, apart the Tomonaga-Luttinger model discussed above and the LutherEmery model reviewed in Section 5.1, the massive and massless Thirring model [87 and the interacting Bose gas 88. Exact solutions are due to a large extent to very strong conservation laws arising from the restricted phase space for 1D fermions.

We briefly discuss some important lattice models. A central role is played by the Hubbard model, and our treatment of Luttinger liquid correlations in lattice models will be centered on this model. We therefore also present a short summary of important Bethe-Ansatz results for this model to make this section more self-contained.

\subsubsection{Models}

The Hubbard model [82] is described by the Hamiltonian

$$
H_{\mathrm{Hub}}=-t \sum_{<i, j>s} c_{i, s}^{\dagger} c_{j, s}+\frac{U}{2} \sum_{i, s}\left(n_{i, s}-1 / 2\right)\left(n_{i,-s}-1 / 2\right)-\mu \sum_{i, s} n_{i, s}
$$

where $c_{i, s}$ describes fermions with spin $s$ in Wannier orbitals at site $i, n_{i, s}=c_{i, s}^{\dagger} c_{i, s}, U$ is the repulsion of two electrons on the same site and $\mu$ the chemical potential. One can also fix the band filling to $n=N_{\text {electrons }} / N_{\text {sites }} .\langle i, j\rangle$ restricts the sum to nearest neighbours. This model is the simplest approximation for strongly correlated electrons in a crystal lattice. The model is exactly solvable, cf. Section 4.4.2. For a long time, it was believed that the Hubbard model describes the strong-coupling limit of the 1D Fermi liquid while the Tomonaga-Luttinger model rather would represent the weak-coupling case. To show that this is not the case, and that both are closely related, is a major purpose of Section 4.4 .

Various more realistic extensions can be considered. In some cases, it is necessary to add longer-range interactions between the electrons. The extended Hubbard model [50, 89]

$$
H_{\mathrm{EHM}}=H_{\mathrm{Hub}}+V \sum_{i} n_{i} n_{i+1}
$$

includes interactions between neighbouring sites, but one may obviously go to longer interaction range [such as $1 / r$ [90] or a Yukawa form $\exp (-r) / r$ ]. In contrast to the Hubbard model, this Hamiltonian is no longer exactly solvable. Also "off-diagonal" terms, i.e. interactions coupling charge densities on site to those on bonds, can be added [91 -96]

$$
H=H_{\mathrm{Hub}}+X \sum_{i, s}\left(c_{i=1, s}^{\dagger} c_{i, s}+\text { H.c. }\right)\left(n_{i,-s}+n_{i+1,-s}\right)
$$

An important feature here is the breaking of charge-conjugation symmetry generated by $X$. This term goes beyond the zero-differential-overlap approximation. A critical 
discussion of the approximations involved in going from a realistic correlation problem to the Hubbard model in a 1D context has been given by Painelli and Girlando [92 and Campbell et al. [94].

At $U>0$ and half-filled band, the Hubbard model has an insulating ground state whose spin fluctuations are described by an effective Heisenberg model [81] with an (antiferromagnetic) exchange integral $J=4 t^{2} / U$. For a nearly half-filled Hubbard model, it is more convenient to think in terms of a few holes doped into such an antiferromagnetic Heisenberg system. For large $U$, double occupancy of lattice sites is dynamically forbidden, and the energy scales for charge fluctuations $(\sim t)$ and for spin fluctuations $(\sim J \ll t)$ are well separated. One can then simplify the problem by projecting out the states in the Hilbert space involving double occupancies. In a restricted Hilbert space containing only singly-occupied and empty sites, one finds in second order in $t / U$ the following Hamiltonian $(t-J$-model, 84- 86])

$$
H_{t-J}=-t \sum_{i}\left[\left(1-n_{i,-s}\right) c_{i, s}^{\dagger} c_{i+1, s}\left(1-n_{i+1,-s}\right)+\text { H.c. }\right]+J \sum_{i}\left[\mathbf{S}_{i} \cdot \mathbf{S}_{i+1}-\frac{1}{4} n_{i} n_{i+1}\right] .
$$

The fermions $c_{i, s}$ now behave as spinless fermions, and $\mathbf{S}_{i}=\sum_{s, s^{\prime}} c_{i, s}^{\dagger}(\sigma)_{s, s^{\prime}} c_{i, s^{\prime}}$ are spin operators. This model can be solved in two limits. For $J=0$, it reduces to the $U=\infty$ Hubbard model which describes free spinless fermions, and for $J / t=2$, it possesses an additional supersymmetry and can be solved by Bethe Ansatz 84]- 86]. The $t-J$-model approximates the strong-coupling limit of the Hubbard model only for $J \ll t$. Models with other interactions or more bands can, however, be approximated in a low-energy subspace by a $t-J$-model with sizable $J$ 97. Both the $t-J$ and the Hubbard model can be extended to include additional degeneracies 98. Another interesting extension consists in introducing longer-range hopping [99] or spin exchange. We shall not say much on these variants here.

Most of the methods discussed below for extracting the Luttinger liquid parameters from one of these models will work, with minor modifications, also for the others with similar structure. When a model is not solvable by Bethe-Ansatz, numerical diagonalization can provide similar information. We therefore limit our discussion as much as possible to the quite generic case of the Hubbard model and only briefly discuss changes occurring when passing to other systems. In the following section, we list some important elements of the Bethe-Ansatz which are helpful for understanding the mapping onto the Luttinger liquid.

\subsubsection{Bethe Ansatz}

The 1D Hubbard model has been solved exactly via Bethe Ansatz by Lieb and Wu 83 (for pedagogical reviews on the Bethe Ansatz, see Sutherland [26], Korepin et al. [27], Izyumov and Skryabin [28] or Nozières [100]), and the ground state energy and some thermodynamic quantities can be obtained [101]-[103]. Also the excitation spectrum of some collective modes has been computed quite early [104, 105. The basic physical picture emerging from these initial studies is as follows. For $U>0$, the system is metallic 
whenever the band is not half-filled ( $N_{\text {electrons }} \neq N_{\text {sites }}=L / a$ with lattice constant $a$ ): the chemical potential for adding a particle to $N$ - and $N-1$-particle systems are equal. Exactly at half-filling, one finds a difference $(\sim \sqrt{U} \exp (-1 / U)$ for $U \rightarrow 0$ and $\sim U$ for $U \rightarrow \infty$ [83, 104]) between these two quantities indicating that the system has turned into a Mott insulator for any $U>0$. Finite $U>0$ obviously prohibits double occupancy of sites, all sites are now (singly) occupied and no low-energy charge excitations possible. The lower Hubbard band is completely filled, and the upper Hubbard band is empty in the ground state. The spins are coupled through an effective antiferromagnetic exchange integral $J=4 t^{2} / U$, and their dynamics reduces to a Heisenberg model.

At half-filling, the $U<0$-sector is related to the $U>0$ one by a particle-hole transformation $c_{i, s} \rightarrow(-1)^{i} c_{i, s}^{\dagger}$ for a single spin direction only, say $s=\uparrow$, exchanging the role of charge and spin degrees of freedom, and the charge gap discussed above turns into a spin gap: occupying sites with two electrons with antiparallel spins is favoured. These pairs are mobile and the charge excitations massless. There are no singular features in the Bethe Ansatz for $U<0$ as a function of band-filling implying that the picture applies to the whole $U<0$-sector [103].

These results can be obtained qualitatively, and for $U / t \ll 1$ also quantitatively, with the renormalization group methods described in the preceding section. The coupling constants are $g_{i \perp}=U a, i=1, \ldots, 4$ ( $g_{3 \perp}$ only occurs for half-filled bands), and the Fermi velocity is $v_{F}=2 t a \sin \left(k_{F} a\right)$.

Bethe's Ansatz provides a solution for all interaction strengths and band-fillings [33]. We sketch the principal ideas, following Nozières [100]. The Bethe Ansatz relies on the following facts. (i) Due to energy and momentum conservation, in 1D a two-particle collision classically and quantum-mechanically conserves both momenta individually. The particles then only can be exchanged or phase-shifted, and the two-particle wave-function asymptotically $\left(\left|x_{1}-x_{2}\right| \rightarrow \infty\right)$ obeys

$$
\Psi\left(x_{1}, x_{2}\right)=a e^{i\left(k_{1} x_{1}+k_{2} x_{2}\right)}+b e^{i\left(k_{1} x_{2}+k_{2} x_{1}\right)} .
$$

The Bethe Ansatz postulates this behaviour for all distances between the particles. (ii) A three-particle collision does not conserve individual momenta except if the scattering matrix factorizes. This factorization implies another conservation law. For $N$ particles, one then has $N$ conservation laws, expressed by $\left\{k_{i}^{\prime}\right\}=\left\{k_{i}\right\}$. (iii) The Hilbert space of the Hamiltonian separates in $N$ ! quadrants each characterized by a permutation $P$ of the $N$ particles, ordered in one quadrant as $1 \leq x_{1} \leq x_{2} \leq \ldots x_{N} \leq L$. The $N$-particle wave-function there becomes

$$
\Psi\left(x_{1}, \ldots, x_{N}\right)=\sum_{P} A[P] e^{i k_{P_{i}} x_{i}} .
$$

Fermi or Bose statistics determines its continuation into the other sectors. (iv) The amplitude $A[P]$ is determined by the conditions of continuity of $\Psi$ as $x_{i} \rightarrow x_{i+1}$ and periodic boundary conditions $\Psi\left(x_{1}, \ldots, x_{N}\right)=\Psi\left(x_{2}, \ldots, x_{N}, x_{1}+L\right)$. The problem is the computation of $A[P]$. (v) Introducing spin, suppose we have $N$ electrons, $M$ of which have spin $\downarrow$, on a lattice with $L$ sites $x_{i}$. One must then ensure that the factorization 
of the $S$-matrix is not perturbed by the spin indices (Yang-Baxter conditions). There is then a second permutation $Q$ for the spin labels, and the wave function where the $M$ down-spins occupy the sites $x_{1} \ldots x_{M}$ and the $N-M$ up-spins the sites $x_{M+1} \ldots x_{N}$ is denoted by $\Psi\left(x_{1}, \ldots, x_{M}, x_{M+1}, \ldots, x_{N}\right)$. The Bethe Ansatz postulates that in each quadrant characterized by $Q$, i.e. $1 \leq x_{Q_{1}} \leq x_{Q_{2}} \leq \ldots x_{Q_{N}} \leq L$, the wave function is given by 83

$$
\Psi\left(x_{1}, \ldots, x_{M}, x_{M+1}, \ldots, x_{N}\right)=\sum_{P} A[Q, P] \exp \left(i \sum_{j=1}^{N} k_{P j} x_{Q j}\right) .
$$

The $N$ numbers $k_{i}$ are determined from the coupled Lieb-Wu equations $(u=U / 4 t)$

$$
\begin{aligned}
2 \pi I_{j} & =L k_{j}-2 \sum_{\beta=1}^{M} \arctan \left(\frac{\sin k_{j}-\Lambda_{\beta}}{u}\right), \\
2 \pi J_{\alpha} & =2 \sum_{j=1}^{N} \arctan \left(\frac{\Lambda_{\alpha}-\sin k_{j}}{u}\right)-2 \sum_{\beta=1}^{M} \arctan \left(\frac{\Lambda_{\alpha}-\Lambda_{\beta}}{2 u}\right), \\
I_{j} & =\left\{\begin{array}{l}
\text { integer } \\
\text { half }- \text { odd }- \text { integer }
\end{array} \quad \text { if } \mathrm{M}=\left\{\begin{array}{l}
\text { even } \\
\text { odd },
\end{array}\right.\right. \\
J_{\alpha} & =\left\{\begin{array}{l}
\text { integer } \\
\text { half }- \text { odd }- \text { integer }
\end{array}\right.
\end{aligned}
$$

The total energy and momentum of the system are then

$$
E=-2 t \sum_{i=1}^{N} \cos k_{i} \quad, \quad P=\sum_{i=1}^{N} k_{i}
$$

Eqs. (4.24) - (4.28) give the exact energy and wavefunction of the 1D Hubbard model. The quantum numbers $k_{i}$ are the momenta of the particles characterizing the orbital degrees of freedom. Unlike for free particles, they are not equally spaced but shifted by the presence of the other particles. The $\Lambda_{\alpha}$ are called rapidities and describe the spin state. On the other hand, the integers or half-odd-integers $I_{i}$ and $J_{\alpha}$ are equally spaced. The ground state is obtained by occupying the levels with minimal $\left|I_{i}\right|$ and $\left|J_{\alpha}\right|$. Therefore the distribution of $q_{i}=2 \pi I_{i} / L$ and $p_{\alpha}=2 \pi J_{\alpha} / L$ is given by a Fermi distribution $\Theta\left(k_{F \uparrow}+k_{F \downarrow}-q_{i}\right)$ and $\Theta\left(k_{F \downarrow}-p_{\alpha}\right)$, respectively. In the absence of a magnetic field, the ground state has $k_{F \uparrow}+k_{F \downarrow}=2 k_{F}$ and $k_{F \downarrow}=k_{F}$, so that the $q_{i}$ have a doubled Fermi wavevector while the $p_{\alpha}$ have the normal $k_{F}$.

This splitting of the Fermi surface into two can be clarified further by studying the elementary excitations. Two of them are obtained by making a hole either in the $I_{i^{-}}$or in the $J_{\alpha}$-distribution. In the first case, one obtains a charged, spinless holon, in the second case a neutral spin-1/2 spinon. Both holon and spinon live in the lower Hubbard band. There are other solitonic excitations involving doubly occupied sites which therefore build up the upper Hubbard band [106]. In general, holons and spinons are not independent, and the Lieb-Wu equations (4.25), (4.26) imply that they interact. Introducing a real hole will affect both channels. Moreover, the representation of physical electrons and holes in terms of holons and spinons is not known to date. 
The interaction of holons and spinons complicates the calculation of their dispersion. Simple results are obtained only for weak or strong $U$. Then, the holons obey

$$
\varepsilon^{(h)}(q)=\left\{\begin{array}{ll}
4 t \cos (q a / 2)-2 t \cos \left(k_{F} a\right) & \text { for } u \ll 1 \\
2 t \cos (q a) & \text { for } u \gg 1
\end{array} .\right.
$$

Their Fermi surface is at $k_{F}^{(h)}=2 k_{F}, \varepsilon_{F}^{(h)}=-\mu$. The spinon have dispersion

$$
\varepsilon^{(s)}(q)=\left\{\begin{array}{ll}
2 t\left[\cos (q a)-\cos \left(k_{F} a\right)\right] & \text { for } u \ll 1 \\
(\pi / 2) J_{e f f} \cos (q a / n) & \text { for } u \gg 1
\end{array} .\right.
$$

The effective exchange integral is

$$
J_{e f f}=\frac{4 t^{2}}{U}\left(n-\frac{\sin (2 \pi n)}{2 \pi}\right) .
$$

The dispersion is only defined for $q \leq k_{F}^{(s)}=k_{F}$, and the energy becomes zero at $k_{F}$. The first feature translates the reduced Brillouin zone of the compressed Heisenberg chain, and the second one implies that spinon-antispinon pairs can be created spontaneously.

The wavefunction (4.24) is not of much practical value due to its enormous complexity: in fact, there are about $N !^{2}$ expansion coefficients $A[Q, P]$ ! In the calculation of the wavefunction, there is no gain with respect to brute-force exact diagonalization. A calculation of correlation functions, and especially of their asymptotic behaviour, based on the Bethe Ansatz is therefore elusive.

Important simplifications occur in the limit $U \rightarrow \infty$ 107. The members on the lefthand sides of Eqs. (4.25) and (4.26) are of order $O\left(U^{0}\right)$. For the equalities to hold, the $\Lambda$ on the right-hand sides must be proportional to $u$ : $\Lambda_{\alpha}=2 u \lambda_{\alpha}$ making the $\sin k_{j}$-terms negligible. This simplifies the Lieb-Wu equations to

$$
\begin{aligned}
2 \pi I_{j} & =L k_{j}+2 \sum_{\beta=1}^{M} \arctan \left(2 \lambda_{\beta}\right), \\
2 \pi J_{\alpha} & =2 N \arctan \left(2 \lambda_{\alpha}\right)-2 \sum_{\beta=1}^{M} \arctan \left(\lambda_{\alpha}-\lambda_{\beta}\right) .
\end{aligned}
$$

The equations for $k_{j}$ and $\lambda_{\alpha}$ now decouple and can be solved successively. Concomitant with this decoupling is a decoupling of the wave function (for the quadrant $Q$ )

$$
\Psi\left(x_{1}, \ldots, x_{M}, x_{M+1}, \ldots, x_{N}\right)=(-1)^{Q} \operatorname{det}\left[e^{i k_{i} x_{Q j}}\right] \Phi\left(y_{1}, \ldots, y_{M}\right)
$$

into a charge and a spin part. det[...] is a Slater determinant involving only the particle positions irrespective of their spin, i.e. describing free spinless fermions. $\Phi\left(y_{1}, \ldots, y_{M}\right)$ is the Bethe Ansatz wave function of a Heisenberg chain 81] of the $N$ spins, characterized through the positions of the $M$ down-spins, on a compressed lattice of just $N$ sites. This decoupling of the wave function means a complete charge-spin separation over all energy scales in the $U \rightarrow \infty$-Hubbard model and is correct to $O(1 / u)$. 
The wave function (4.34) can be evaluated numerically for much bigger systems than (4.24) and, combined with either finite-size scaling or further analytical work, allows to discuss the asymptotic low-energy properties and the critical exponents of correlation functions. Applications will be discussed in the following section. We also note that there is a systematic large- $U$ expansion for the distribution functions of the momenta $k$ and rapidities $\Lambda$ [108].

\subsubsection{Low-energy properties of one-dimensional lattice models}

In the first part of this section, we shall discuss various successful methods to derive the correlation exponents of interacting electrons in 1D lattices, taking the Hubbard model as an example. At the end, we briefly summarize the physical picture and then outline the changes occurring when going to the variants introduced in Section 4.4.1.

Early studies of correlation functions heavily relied on numerical simulation of Hubbard and extended Hubbard models. Hirsch and Scalapino used quantum Monte Carlo techniques to directly study the density and spin density correlation functions at various band-fillings and interaction strengths on lattices of 20 - 40 sites at temperatures down to about $t / 15$ [109. Of course, both the finite temperatures and the accuracy of the simulations did not allow a determination of the correlation exponents and thus of $K_{\rho}$, but one main point of concern was the doubling of the wave vector of divergence in the charge density response from $2 k_{F}$ to $4 k_{F}$ as interactions are increased and/or $V$ is turned on. This could be rephrased in terms of the present language as under what conditions $K_{\rho}+1<(>) 4 K_{\rho}$ i.e. $K_{\rho}<(>) 1 / 3$ which marks the value where $2 k_{F^{-}}$and $4 k_{F^{-}}$-responses are equally divergent. Quite generally, it was found that increasing $U$ decreases the $2 k_{F^{-}}$ CDW-correlations but somewhat increases the $4 k_{F^{-}} \mathrm{CDW}$ as $U \rightarrow \infty$. The decrease at $2 k_{F}$ was less, however, if charge density fluctuations were measured on the bonds between lattice sites (bond order wave, BOW) rather than on the lattice sites themselves. The $2 k_{F}$-SDW correlations were enhanced by $U$. This is quite easy to understand physically: $U$ generates antiferromagnetic spin exchange but suppresses on-site charge fluctuations while intersite fluctuations remain unaffected, at least at lowest order in $U$. On the other hand, for $U \rightarrow \infty$, the electrons behave as spinless fermions with a Peierls divergence

vector of $2 k_{F}^{(s=0)}=4 k_{F}^{(s=1 / 2)}$. Adding a nearest-neighbour repulsion $V$ strongly favours the $4 k_{F}$-CDW, especially on-site and in quarter-filled bands, also enhances the SDW and further suppresses the $2 k_{F}$-CDW: the energies due to both $U$ and $V$ are minimized when the particles occupy every second site, i.e. forming a $4 k_{F^{-}} \mathrm{CDW}$.

The suppression of the $2 k_{F}$-CDW by $U$ and $V$ is interesting in view of the general expression for the density correlations in a Luttinger liquid, Eqs. (3.92) and (3.102) which imply that both exponents of divergence at $2 k_{F}$ and $4 k_{F}$ increase with decreasing $K_{\rho}$ and thus with increasing $U$ and $V$. The suppression of the $2 k_{F^{-}}$CDW then must be due to the influence of $U$ on its prefactor which must decrease as $U$ increases. Hirsch and Scalapino demonstrate this by showing that, at low enough temperature and various $U$, both SDW and CDW diverge with the same exponent but that the scale where the asymptotic behaviour is observed, is vastly different and, in fact, very low for the CDWs [109. A related 
suppression of $2 k_{F}$-CDW correlations due to the prefactor (with a concomitant enhancement of SDW and BOW) can also demonstrated for a half-filled band in renormalization group [78].

More extended results on the influence of band-filling and interaction range on the competition of $2 k_{F^{-}}$and $4 k_{F^{-}}$CDWs have been produced by Mazumdar, Dixit, and Bloch [110]. They also propose a qualitative but systematic picture predicting the appearance of $2 k_{F^{-}}$or $4 k_{F^{-}}$CDWs, in terms of the contribution to the ground state wave function of certain extreme symmetry broken configurations and the barriers to resonance between them. Specifically, for the quarter-filled band, finite $V$ is necessary to promote a $4 k_{F^{-}}$ CDW but an eventual second-neighbour repulsion $V_{2}$ must be small: $V_{2}<V / 2$. For $1 / 2<n<2 / 3$, however, a new kind of defect-CDW with periodicity $\pi / a$ is found possible and competes with the $4 k_{F}$ one, depending on the precise values of the interaction constants. Long-range interactions are necessary to stabilize a $4 k_{F^{-}} \mathrm{CDW}$ for $n>2 / 3$, and the generic CDW will be at $2 k_{F}$. The competition between $2 k_{F^{-}}$and $4 k_{F^{-}}$CDWs will reappear below in terms of the Luttinger parameter $K_{\rho}$ being smaller or larger than $1 / 3$. For the special case of $n=1 / 2$, electron-phonon coupling has also been included recently [111]. Also, a more systematic theory for a Luttinger liquid floating on top of a commensurate CDW, e.g. with $q_{C D W}=\pi / a$, will be given at the end of this section [112].

Subsequent work rather turned attention to single-particle properties and to the question of (non)-Fermi-liquid behaviour in the 1D Hubbard model. Several quantum Monte Carlo studies indicated a finite jump in the momentum distribution function $n(k)$ at $k_{F}$, to be compared with the Luttinger prediction (3.86) 113-116. Notice, however, that Equation (3.86) applies to an infinite system. Finite size effects give $n(k)$ a finite jump at $k_{F}$ whose scaling is governed by the exponent $\alpha$ [57, 115]

$$
\Delta n\left(k_{F}\right)=n\left(k_{F}-\pi / L\right)-n\left(k_{F}+\pi / L\right) \sim L^{-\alpha},
$$

subsequently identified in improved simulations [117]. The absence of any significant rounding expected from the power-law behaviour in $n(k)$, up to about 200 lattice sites indicates, however, that the asymptotic Luttinger regime in the 1D Hubbard model is confined to a tiny momentum slice around the Fermi surface, whose smallness, in fact, remains surprising. (With reference to the different scales in different quantities, identified by Hirsch and Scalapino [109] and discussed above, this does not necessarily imply that Luttinger liquid correlations in all other quantities are confined to such small momentum/energy scales.)

Sorella et al. also studied the divergences of the density and spin density correlation functions of the 1D Hubbard model and could identify the different exponents from finitesize scaling [117. In particular, they were able to verify the scaling relations between $\alpha_{C D W}, \alpha_{S D W}$, and $\alpha_{4 k_{F}}$, Eqs. (3.92) - (3.98), implied by their dependence upon $K_{\rho}$ alone ( $K_{\sigma}=1$ for $\mathrm{SU}(2)$-invariance), and they found an upper limit $\alpha=1 / 8$ as $U \rightarrow \infty$, implying $K_{\rho} \geq 1 / 2$.

These exponents can be determined exactly from the $U \rightarrow \infty$ Bethe wave function 
[107. We study the momentum distribution function

$$
n(k)=\left\langle c_{k s}^{\dagger} c_{k s}\right\rangle=\frac{1}{L} \sum_{j, l}\left\langle c_{j s}^{\dagger} c_{l s}\right\rangle e^{i k(j-l) a} .
$$

At this stage, the real-space representation of the Bethe wave function can be used. In order to transfer an electron from site $l$ to $j$, we have to take out of the Slater determinant one spinless fermion at $l$ and reinsert it at $j$. At the same time, the spin configuration is changed: we must take out of the Heisenberg chain the spin at $l^{\prime}$, corresponding to the electron at $l\left(l \neq l^{\prime}\right.$ because of the compressed lattice), and insert it again at $j^{\prime}$ corresponding to the new electron site $j$ which can also be viewed as permuting neighbouring spins successively between $l^{\prime}$ and $j^{\prime}$. Then, one has to sum over all configurations of the spinless fermions, as implied by the average $\langle\ldots\rangle$ in (4.36) taken over the wavefunction (4.34). The permutation of two neighbouring spins is mediated by the operator $2 \mathbf{S}_{i} \cdot \mathbf{S}_{i+1}+1 / 2$ so that the evaluation of the spin contribution to $n(k)$ requires calculation a correlation function of $j-l$ of these operators for each configuration of spinless fermions. The charge contribution, on the other hand, is just the product of two Slater determinants.

Ogata and Shiba solved these functions numerically and obtained a function $n(k)$ characterized by a jump at $k_{F}$ and, surprisingly at that time, another weak singularity at $3 k_{F}$, shown in Fig. 4.3. Both of these facts were somewhat surprising because the spinless fermions' $n(k)$ jumps at $2 k_{F}$ which thus appeared a natural candidate for Fermi surface of the $U \rightarrow \infty$-Hubbard model. However, the electrons involved in $n(k)$ both contain a charge and a spin component, and the spins feed back into the charges by the kernel on the right-hand side of (4.32). The (re-)appearance of $k_{F}$ and $3 k_{F}$ is due to oscillations with wavevector $\pm 2 k_{F}$ in the spin contribution to $n(k)$. A careful finite-size-scaling analysis showed that the apparent jump at $k_{F}$ would fade away as $L \rightarrow \infty$ and that the variation of $n(k)$ with system size was compatible with the Luttinger liquid power law (3.86) with an exponent $\alpha \approx 0.14$. The $3 k_{F}$-singularity was shown to be due an excitation of $k_{F}$-fermions to $3 k_{F}$ accompanied by creation of electron-hole pairs with $-2 k_{F}$, and is also required by the picture of Section 4.2. They also studied the spin-spin correlation function at $q=2 k_{F}$. From the singularity observed as a function of $q$ they inferred a decay in real space as $R_{S D W}(x) \sim \cos \left(2 k_{F} x\right) x^{-1.44}$ while their results for the Heisenberg model were consistent with the known form $\sim \cos (\pi x) \ln ^{1 / 2}(x) / x$ [79, 118.

An analytical evaluation of these quantities is also possible [119]-[121]. Parola and Sorella started from an evaluation of the spin-spin correlation function [119]

$$
<\mathbf{S}_{r} \cdot \mathbf{S}_{0}>=\sum_{j=2}^{r+1} P_{S F}^{r}(j) S_{H}(j-1),
$$

where $S_{H}(j)$ is the (known) spin correlation function of the Heisenberg model and $P_{S F}^{r}(j)$ is the probability of finding $j$ (spinless) particles between 0 and $r$ with one at 0 and one at $r$. The evaluation of this latter quantity is difficult but at least asymptotically possible, and one finds

$$
<\mathbf{S}_{r} \cdot \mathbf{S}_{0}>\approx \cos \left(2 k_{F} r\right) \frac{\ln ^{1 / 2} r}{r^{3 / 2}}
$$


which is consistent with the Luttinger liquid function (3.98) provided $K_{\rho}=1 / 2$. This implies an exponent $\alpha=1 / 8$ for the momentum distribution function $n(k)$ of the $U=\infty$ Hubbard model at $k_{F}$, in quite good agreement with the numerical data of Ogata and Shiba. Parola and Sorella could recalculate analytically the momentum distribution, following the procedure by Ogata and Shiba and, fixing two open parameters so as to reproduce the behaviour at $k_{F}$, were able to identify the exponent at $3 k_{F}$ as $\alpha_{3 k_{F}}=9 / 8$ [120], a value also found by others [65, 122].

Anderson and Ren compute the correlation exponents of the Hubbard model in the $U \rightarrow \infty$-limit in a more physical way 122 . They observe that the Ogata-Shiba wave function implies charge-spin separation only for those excitations which take place solely in one channel. If we consider correlation functions of excitations affecting both channels, phase shifts will arise in the distribution of the momenta $\left\{k_{i}\right\}$ due to changes in the rapidity-distribution. This is due to the kernel on the right-hand side of Eq. (4.32), and to the parity effects in the distributions of the quantum numbers $I_{i}$ and $J_{\alpha}$ in the LiebWu equations, cf. (4.27). As an example, the Green function for the holon at $+2 k_{F}$ is $G^{(h)}(x, t) \sim e^{2 i k_{F} x} /\left(x-v_{\rho} t\right)$ and involves only charge degrees of freedom: if one adds an $I_{i}$ to the system, the rapidities do not change. Removing a spinon at $-k_{F}$, i.e. a $J_{\alpha}$, there is a phase shift of $\delta_{ \pm 2 k_{F}}=\pi / 2$ of all holon momenta in the same direction. The spinon Green function, which for the Heisenberg model is $e^{i k_{F} x} / \sqrt{x-v_{\sigma} t}$, will therefore also contain a contribution from the phase shift of the holons which reduces the overlap with the "unshifted" ground state wave function. This introduces a factor $\left(x \pm v_{\rho} t\right)^{-(\delta / 2 \pi)^{2}}$ for the phase shifts on each side of the momentum distribution. The $2 k_{F}$-spin-spin correlation function consists of a right-moving particle (spinon) and a left-moving hole (antispinon). Each of them shifts the $k_{i}$-distribution in the same direction, so that the total phase shift is $\delta_{ \pm 2 k_{F}}=\pi$ on each side. Putting everything together, we have

$$
\begin{aligned}
\langle\mathbf{S}(x t) \cdot \mathbf{S}(00)\rangle & =\frac{\cos \left(2 k_{F} x\right)}{\left(x-v_{\sigma} t\right)^{1 / 2}\left(x+v_{\sigma} t\right)^{1 / 2}\left(x-v_{\rho} t\right)^{1 / 4}\left(x+v_{\rho} t\right)^{1 / 4}} \\
& =x^{-3 / 2} \cos \left(2 k_{F} x\right) \text { for } t=0 .
\end{aligned}
$$

The $4 k_{F^{-}}$CDW only involves a right-moving holon and a left-moving antiholon, and therefore is decoupled from the spins

$$
\left\langle O_{4 k_{F}}(x t) O_{4 k_{F}}^{\dagger}(00)\right\rangle=\frac{\cos \left(4 k_{F} x\right)}{\left(x-v_{\rho} t\right)\left(x+v_{\rho} t\right)} .
$$

Other interesting examples are provided by the $k_{F^{-}}$and $3 k_{F}$-pieces of the single-particle Green function. Here, one has to take out (add) both a holon and a spinon. One can take out the holon at $2 k_{F}$ and the spinon at $-k_{F}$. The removal of the spinon shifts the holon momenta by $\pi / 2$ in the positive direction, and this phase shift cancels a quarter of the $2 \pi$-shift caused by the holon removal at $2 k_{F}: \delta_{2 k_{F}}=3 \pi / 2$ while $\delta_{-2 k_{F}}=\pi / 2$. This process determines the $k_{F}$-component. One can, however, also take out the spinon at $+k_{F}$, and then the ensuing phase shift adds to that of the holon removal $\delta_{2 k_{F}}=5 \pi / 2$. This determines the $3 k_{F}$-Green function. We have

$$
G_{k_{F}\left(3 k_{F}\right)}(x t)=\frac{\exp \left[i\left(2 k_{F} \pm k_{F}\right) x\right]}{\left(x-v_{\sigma} t\right)^{1 / 2}\left(x-v_{\rho} t\right)^{(1 \mp 1 / 4)^{2}}\left(x+v_{\rho} t\right)^{1 / 16}} .
$$


All these correlation functions agree with the Luttinger liquid expressions taken at $K_{\rho}=$ $1 / 2$.

The removal or addition of spinons and holons can also be interpreted in terms of the charge and current excitations of a Luttinger liquid in the charge and spin channels. One can consider the $3 k_{F}$-component of the Green function as being due to an additional current excitation with momentum $2 k_{F}$ with respect to the $k_{F}$-piece. Anderson and Ren also prove that the correlation exponents of the Green function, which can be derived from the kernels of the Lieb-Wu equations (4.25) and (4.26) [75, are precisely the Fermi-surface phase shifts due to the insertion of an additional particle 122.

The applicability of these methods is, however, quite restricted: (i) there are many models which cannot be solved exactly, and (ii) even if a Bethe Ansatz solution is available, manageable simplifications generally only occur in special limits such as $U \rightarrow \infty$ for the Hubbard model. On the other hand, the notion of a Luttinger liquid is based on the low-energy properties of a many-body problem, and a priori, a complete solution is not required. Following Haldane [49] and Section 3.2.2, a Luttinger liquid can be identified and its characteristic parameters determined by using only the low-energy spectrum of the lattice Hamiltonian. These can be found reliably either from an exact solution or by numerical diagonalization.

One general method is due to Efetov and Larkin [10] and Haldane [3, 49] where it was formulated for a spinless fermion system, and then extended by Schulz [42, 48] for models of $S=1 / 2$-electrons. Here, one formulates an effective Luttinger Hamiltonian for the low-energy physics and then identifies its parameters $K_{\nu}, v_{\nu}$ from the properties of the exact solution. Central to this approach is the use of the relations between the correlation exponents $K_{\nu}$ and the renormalized velocities [49] $v_{N \nu}=v_{\nu} / K_{\nu}, ; v_{J \nu}=v_{\nu} K_{\nu}$, Eq. (3.32). To identify the Luttinger liquid one must, in principle, determine the three velocities $v_{\nu}, v_{N \nu}$, and $v_{J \nu}$ per degree of freedom and check that they satisfy (3.32). $K_{\nu}$ is then obtained automatically. In practice, this programme is rarely carried out to this point (with the notable exception of [75]). Rather, one assumes (3.32) to hold and determines both $v_{\nu}$ and $v_{N \nu}$ which are sufficient to yield the remaining parameters $K_{\nu} \cdot v_{N \rho}=v_{\rho} / K_{\rho}$ is related to the compressibility $\kappa$ by Eq. (3.62) $\kappa^{-1}=L^{-1} \partial^{2} E / \partial n^{2}=\pi v_{N \rho} / 2$, and the change of the ground state energy with particle density $n$ can be readily determined by Bethe Ansatz or numerical methods. A similar relation (3.62) for $v_{N \sigma}=v_{\sigma} / K_{\sigma}$ to the magnetic susceptibility can be explored in the spin sector. If the system is spinrotation invariant, $K_{\sigma}=1$ and $v_{\sigma}$ is found. In the charge sector, $v_{\rho}$ must be determined independently from the low-energy spectrum of charge excitations. To identify which type of excitations in the Bethe Ansatz is relevant, one can realize, as does Schulz [42], from the boson representation (3.90) that the $4 k_{F}$-CDW operator involves only charge degrees of freedom and then argue that power-law decay of this correlation function must originate from gapless excitations at that wavevector. These "particle-hole" excitations have been known since a long time [105], and their velocity is

$$
v_{\rho}=\lim _{p \rightarrow 0} \frac{\varepsilon\left(k_{0}, p_{0}\right)}{p\left(k_{0}, p_{0}\right)} \text {. }
$$


Operationally, in the Bethe Ansatz wave function, take one particle with pseudo-momentum $k_{0}$ out of the filled (charge) pseudo-Fermi sea and put it into one of the empty states above at pseudo-momentum $p_{0}$. Find the energy $\varepsilon\left(k_{0}, p_{0}\right)$ and (physical) momentum $p\left(k_{0}, p_{0}\right)$ associated with this excitation; then take the limit $p \rightarrow 0$. This gives $v_{\rho}$, and $K_{\rho}$ is then determined, too. Ultimately, the correlation exponent $K_{\rho}$ is fully determined by thermodynamic properties [3, 10, 48, 49].

This procedure does not suffer from the limitations of perturbative renormalization group and allows an exact calculation of the Luttinger liquid parameters. Being related to properties of the eigenvalue spectrum, it can easily be adapted to numerical exact diagonalization studies. One concern might be finite size effects because the numerical solutions are confined to small systems. They are, however, not critical here since the energies of the low-lying states usually converge rather quickly to the infinite system limit.

One can also apply conformal field theory methods to determine the correlation exponents of the Hubbard model. Conformal field theory, as we have sketched it in Section 3.6, requires a Lorentz-invariant system [29]. This is the case for spinless fermions (or models of the same universality class) with only one branch of gapless excitations, and has consequently been applied to such problems with great success [74. The Hubbard model and all other models in the universality class of a spin-1/2 Luttinger liquid are not Lorentz-invariant because the spin and charge velocities $v_{\sigma} \neq v_{\rho}$ play the roles of two different velocities of light. Each channel $\nu$ taken by itself is conformally invariant, however, described by a Virasoro algebra with central charge $c_{\nu}=1$. The complete theory is then described by a semidirect product of these Virasoro algebras, and the scaling dimensions of operators now depend, instead of a single coupling constant, on an $N \times N$-matrix of coupling constants, the "dressed charge matrix", for an $N$-component system [123].

Frahm and Korepin have applied these methods to the 1D Hubbard model in order to deduce the long-distance asymptotics of its correlation functions [124. The idea is the following: the elements of the dressed charge matrix

$$
Z \equiv\left(\begin{array}{cc}
Z_{c c} & Z_{c s} \\
Z_{s c} & Z_{s s}
\end{array}\right)=\left(\begin{array}{ll}
\xi_{c c}\left(k_{0}\right) & \xi_{c s}\left(\Lambda_{0}\right) \\
\xi_{s c}\left(k_{0}\right) & \xi_{s s}\left(\Lambda_{0}\right)
\end{array}\right)
$$

(and, of course, the velocities of the gapless excitations $v_{\nu}$ ) can be evaluated from the Bethe Ansatz. Its entries $\xi$ obey equations derived from and similar to the Lieb-Wu equations (4.25), (4.26), with the limit $L \rightarrow \infty$ taken. $k_{0}$ and $\Lambda_{0}$ are the cutoffs in the distribution functions of the momenta and rapidities. The entries of the dressed charge matrix are related to thermodynamic quantities of the model in much the same way as the effective coupling constant of spinless fermions is. For example, Frahm and Korepin find 124

$$
\xi_{c c}^{2}\left(k_{0}\right)=\pi v_{\rho} n^{2} \kappa
$$

where $\kappa$ is the compressibility and $n$ the charge density. Comparing (4.44) to (3.62), we see that $\xi_{c c}^{2}\left(k_{0}\right)$ is essentially $K_{\rho}$, up to a factor $n^{2}$. This formula has been derived independently by Kawakami and Yang [125], who use earlier Bethe Ansatz evaluations of on the thermodynamic properties in order to get $\xi_{c c}$ as a function of the system parameters. These authors, however, neglect the off-diagonal elements of the dressed charge matrix. 
In analogy to Section 3.6, conformal invariance then determines the scaling dimensions of (primary and descendant) operators. The role of the coupling constant $g$ of the Gaussian model is now played by the matrix $Z . \Delta N_{c(s)}$ and $D_{c(s)}$, later grouped into vectors $\Delta \mathbf{N}$ and $\mathbf{D}$, count the charges $(c)$ and spins $(s)$ added by the field $\phi$ to the Bethe Ansatz distribution and are (up to linear combination) the changes in the charge and current excitations of the Luttinger model [126]. The ground state has $\mathbf{\Delta N}=\mathbf{D}=\mathbf{0}$. Allowed values of $\mathbf{D}$ depend on $\boldsymbol{\Delta} \mathbf{N}$. For example, for a fermion operator $c_{ \pm k_{F}, \uparrow}^{\dagger}, \boldsymbol{\Delta} \mathbf{N}=(1,0)$, $\mathbf{D}=( \pm 1 / 2, \mp 1 / 2)$, for $c_{ \pm k_{F}, \downarrow}^{\dagger}, \boldsymbol{\Delta} \mathbf{N}=(1,1), \mathbf{D}=(0, \pm 1 / 2)$, and for the density operator $\rho$, one has $\Delta \mathbf{N}=\mathbf{0}$. Numbers $N_{c(s)}^{ \pm}$characterize descendent fields $\phi$ at level $\sum N_{c(s)}^{ \pm}$. Primary fields have $N_{c, s}^{ \pm}=0$, and finite values describe secondary fields. The correlation functions of the primary and descendent fields $\phi_{\Delta^{ \pm}}$with scaling dimensions $\Delta^{ \pm}$are given by conformal field theory as

$$
\left\langle\phi_{\Delta^{ \pm}}(x, t) \phi_{\Delta^{ \pm}}(0,0)\right\rangle=\frac{\exp \left[-2 i D_{c} P_{F, \uparrow} x\right] \exp \left[-2 i\left(D_{c}+D_{s}\right) P_{F, \downarrow} x\right]}{\left(x-i v_{\rho} t\right)^{2 \Delta_{c}^{+}}\left(x+i v_{\rho} t\right)^{2 \Delta_{c}^{-}}\left(x-i v_{\sigma} t\right)^{2 \Delta_{s}^{+}}\left(x+i v_{\sigma} t\right)^{2 \Delta_{s}^{-}}}
$$

which is a direct generalization of (3.163) to a two-component system.

As in Section 3.6, the central charge and the scaling dimensions can be obtained from the finite size corrections to the energy of the ground state and the energies and momenta of low-lying excited states of the system. For $c=1$ in both the charge and spin channel, (3.157) generalizes to

$$
E_{0}(L)-L \varepsilon_{0}=-\frac{\pi}{6 L}\left(v_{\rho}+v_{\sigma}\right)+\mathcal{O}\left(\frac{1}{L}\right),
$$

where the symbol $\mathcal{O}(1 / L)$ stands for terms decaying faster than $1 / L$. $E_{0}$ is the ground state energy at size $L$, and $\varepsilon_{0}$ is the energy density in the infinite system. Eq. (4.46) must be verified by the solution. Eqs. (3.161) and (3.162) for energy and momentum of the low-lying excitations are given by

$$
\begin{aligned}
& E(\boldsymbol{\Delta} \mathbf{N}, \mathbf{D})-E_{0}=\frac{2 \pi}{L}\left[v_{\rho}\left(\Delta_{c}^{+}+\Delta_{c}^{-}\right)+v_{\sigma}\left(\Delta_{s}^{+}+\Delta_{s}^{-}\right)\right]+\mathcal{O}\left(\frac{1}{L}\right) \\
& P(\boldsymbol{\Delta} \mathbf{N}, \mathbf{D})-P_{0}=\frac{2 \pi}{L}\left(\Delta_{c}^{+}-\Delta_{c}^{-}+\Delta_{s}^{+}-\Delta_{s}^{-}\right)+2 D_{c} P_{F \uparrow}+2\left(D_{c}+D_{s}\right) P_{F \downarrow} .
\end{aligned}
$$

On the other hand, these quantities can be computed from the Bethe Ansatz (or numerically) as

$$
\begin{gathered}
E(\Delta \mathbf{N}, \mathbf{D})-E_{0}=\frac{2 \pi}{L}\left\{\frac{1}{4} \Delta \mathbf{N}^{T} \cdot\left(Z^{-1}\right)^{T} \cdot\left(\operatorname{diag}\left[v_{\rho}, v_{\sigma}\right]\right) \cdot Z^{-1} \cdot \Delta \mathbf{N}+\right. \\
\left.+\mathbf{D}^{T} \cdot Z \cdot\left(\operatorname{diag}\left[v_{\rho}, v_{\sigma}\right]\right) \cdot Z^{T} \cdot \mathbf{D}+v_{\rho}\left(N_{c}^{+}+N_{c}^{-}\right)+v_{\sigma}\left(N_{s}^{+}+N_{s}^{-}\right)\right\}+\mathcal{O}\left(\frac{1}{L}\right),
\end{gathered}
$$

$P(\Delta \mathbf{N}, \mathbf{D})-P_{0}=\frac{2 \pi}{L}\left\{\Delta \mathbf{N}^{T} \cdot \mathbf{D}+N_{c}^{+}-N_{c}^{-}+N_{s}^{+}-N_{s}^{-}\right\}+2 D_{c} P_{F, \uparrow}+2\left(D_{c}+D_{s}\right) P_{F, \downarrow}$.

Comparing Eqs. (4.48) to (4.47) one deduces the scaling dimensions

$$
\begin{aligned}
& 2 \Delta_{c}^{ \pm}(\Delta \mathbf{N}, \mathbf{D})=\left(Z_{c c} D_{c}+Z_{s c} D_{s} \pm \frac{Z_{s s} \Delta N_{c}-Z_{c s} \Delta N_{s}}{2 \operatorname{det} Z}\right)^{2}+2 N_{c}^{ \pm}, \\
& 2 \Delta_{s}^{ \pm}(\Delta \mathbf{N}, \mathbf{D})=\left(Z_{c s} D_{c}+Z_{s s} D_{s} \pm \frac{Z_{c c} \Delta N_{s}-Z_{s c} \Delta N_{c}}{2 \operatorname{det} Z}\right)^{2}+2 N_{s}^{ \pm} .
\end{aligned}
$$


In order to obtain the correlation functions of the Hubbard model, on would have to expand the physical operators $O$ in terms of the conformal fields. This is usually not possible. On the other hand, the quantum numbers of the intermediate states can be determined from the representation of the operators $O$ in terms of the electron creation and annihilation operators. We have given examples above. Then, the complete asymptotic behaviour of the correlation functions can be given in terms of the scaling dimensions and thus in terms of the dressed charge matrix $Z$ whose entries depend on $U$ and the bandfilling $n$. As an example, the first few terms of the complete density-density correlation function are given by Frahm and Korepin [124] and, in the absence of a magnetic field, reduce to Eqs. (3.91)-(3.93) when the appropriate $K_{\rho}$ is inserted there. In this way, the conformal fields contributing to the density-density correlations are not explicitly identified. The knowledge of their scaling dimensions is sufficient to determine their contribution to the correlation function. Penc and Solyom have finally deduced explicit Tomonaga-Luttinger coupling constants $g_{i}$ from the dressed charge matrix and the scaling dimensions of the Hubbard model [126].

While the asymptotic correlation exponents agree with the approach by Schulz 42, 48] and Kawakami and Yang [125, there are some subtle differences. In general, in the absence of magnetic fields, $Z$ is not diagonal as naively expected for charge-spin separation. However, one of the matrix elements $Z_{c s}=0$ and $Z_{s c}=Z_{c c} / 2$ which gives critical exponents identical to those for a charge-spin separating system as assumed by Schulz. One can include an external magnetic field [127]. Then, there is no longer a simple relation between the elements of $Z$, the exponents now differ from those derived under the assumption of charge-spin separation, and charge and spin are strongly coupled. On the other hand, the dressed charge matrix is probably not a good quantity to "measure" charge-spin separation, because it does not change in any essential way in the limit $U \rightarrow \infty$ where we know 107] that the product form of the Bethe wavefunction implies complete charge-spin separation.

The dependence of $K_{\rho}$ on $U$ and $n$ is shown in Fig. 4.4, and that of the velocities $v_{\nu}$ in Fig. 4.548 . For small $U$, the variation of $K_{\rho}$ with $U$ is consistent with the perturbative result $K_{\rho} \approx 1-U / \pi v_{F}$, and the slope varies with bandfilling due to the $n$-dependence of the Fermi velocity $v_{F}=2 t \sin (\pi n / 2)$. At larger $U, K_{\rho}$ deviates from a straight line and $K_{\rho} \rightarrow 1 / 2$ for $U \rightarrow \infty$ for all $n$. $K_{\rho}=1 / 2$ is also the limit for $n \rightarrow 0$ for any $U>0$ which is quite obvious due to the $n$-dependence of $v_{F}$. Also $K_{\rho} \rightarrow 1 / 2$ for $n \rightarrow 1,(U>0)$, cf. below. The velocities $v_{\nu} \rightarrow v_{F}$ for $U \rightarrow 0$ as expected, and as $U \rightarrow \infty, v_{\rho}=2 t \sin (\pi n)$ and $v_{\sigma}=\left(2 \pi t^{2} / U\right)[1-\sin (2 \pi n) /(2 \pi n)]$. While $v_{\rho} \propto n$ for all $U$ and small $n, v_{\sigma} \propto n^{2}$ for $U>0$ and $\propto n$ for $U=0$.

These parameters can then be inserted into the results obtained in Section 3.3 to obtain the correlation functions of the Hubbard model as a function of $U$ and $n$. In particular, for $U \rightarrow \infty$, one obtains $\alpha \rightarrow 1 / 8, \alpha_{C D W, S D W} \rightarrow 1 / 2$ and $\alpha_{4 k_{F}} \rightarrow 0$. The properties of the charge degrees of freedom in this limit can be straightforwardly understood in terms of spinless fermions, in agreement with the factorization of the Bethe wave function. E.g. the $4 k_{F}$-part of the density-density correlations is simply the $2 k_{F}$-CDW of free spinless fermions with a doubled Fermi wavevector. The large- $U$ limit of $v_{\rho}$ is simply the Fermi 
velocity of free spinless fermions with a hopping integral $t$. Also close to half-filling even at finite $U$, a spinless fermion picture applies: here one best thinks in terms of a few holes doped into the insulating half-filled band, and the repulsion $U$ is accounted for by treating them as spinless fermions. When there are very few of them, their mutual interaction will be negligible. This explains the value $K_{\rho}=1 / 2$ found for all $U$ as $n \rightarrow 1$. The spinless fermion picture also implies that the prefactor of the $2 k_{F}$-part of the densitydensity correlation function must vanish as $U \rightarrow \infty$. More care has to be taken for spin or single-particle correlations. The ground state of the Hubbard model can be viewed as containing a number of holons appropriate to the doping level but no spinons. In this way, it becomes clear that the characteristic wave vector for the SDW oscillations $2 k_{F}$ shifts with doping due to the introduction of holes although in a local picture, there are no configurations of parallel neighbouring spins [42]. The motion of holons disrupts the spin correlations and therefore leads to a more rapid decay of the spin-spin correlations than in the half-filled band or a Heisenberg antiferromagnet. Introducing a hole (or an electron) creates, however, a holon at $\pm 2 k_{F}$ and a spinon at $\pm k_{F}$, and therefore the single-particle Green function oscillates with wavevectors $k_{F}, 3 k_{F}$, etc.

The low-energy spectral function of the Hubbard model, obtained by inserting $\alpha=$ $1 / 8$ for the limit $U \rightarrow \infty$ into the Luttinger model, is shown in Fig. 3.6. It is clearly dominated by the spectral weight between $v_{\sigma}$ and $v_{\rho}$, and the weight above/below $\pm v_{\rho}$ is quite negligible. Comparison to functions of models with either charge-spin separation or anomalous dimensions only, suggests that charge-spin separation is the dominant nonFermi-liquid feature in the 1D Hubbard model [53, 59, 60]. Even for infinite repulsion, the anomalous correlations are quite weak. Physically, this implies that the power-laws in the correlations are most sensitive to the range of the interaction, taken finite in the Luttinger but zero in the Hubbard model, while the influence of short-range interactions is strong on charge-spin separation. This allows to rationalize the small momentum range, where Luttinger liquid behaviour is seen in $n(k)$ in Figure 4.3. Unfortunately, no signature of charge-spin separation has been detected in a Monte-Carlo simulation of the spectral function directly of the Hubbard model [128. This could be due to finite system size and/or temperature, but certainly needs further study.

Correlation functions of the $t-J$-model behave in a similar manner and also identify it as a Luttinger liquid [129, 130. Specifically, at the supersymmetric point $J / t=2$, where the model is solvable by Bethe Ansatz, conformal field theory allows to derive the dependence of $K_{\rho}$ on band-filling [129], in a similar manner as for the Hubbard model. It obeys to the same limits as for the $U>0$ Hubbard model $1 \geq K_{\rho} \geq 1 / 2$ but tends towards the free value for the nearly empty band, while in this limit the Hubbard model behaves as if $U \rightarrow \infty$. On going away from the supersymmetric point, the model is no longer solvable, and one has to turn to numerical diagonalization on small clusters to obtain the correlation exponents [130. Again, one uses Eq. (3.62) to obtain $v_{\rho} / K_{\rho}$ and separately studies the spectrum of the charge excitations. While $K_{\rho}$ continues to obey to the lower bound $K_{\rho} \geq 1 / 2$ (the equality holding for empty bands at $J<2 t$, half-filled bands at $J<3.5 t$ and at $J=0$ for any filling), $K_{\rho}>1$ now occurs for larger values of $J$. Eqs. (3.99) and (3.100) imply a region of dominant superconducting 
fluctuations. According to the general scaling arguments above, logarithmic corrections would favour the triplet type if the spins are massless, while opening of a spin gap would make singlet superconductivity dominate. Evidence for a spin gap has been produced by using variational wavefunctions, a procedure to be discussed below [131]. Imada and Hatsugai also measured spin correlation functions in their Monte Carlo simulations [116]. While for small $J / t$, their results are quite close to those of the Hubbard model found by Hirsch and Scalapino [109], the spin correlations become commensurate, i.e. peaked at $q=\pi / a$ rather than at $2 k_{F}$ as $J$ increases. In this regime, the holes in the $t-J$-model probably act as mobile defects in a short-range-ordered antiferromagnetic background. Finally, in the large- $J$ region $(>2 \ldots 3.5 t$ depending on $n$ ), phase separation occurs: here the attraction due to the interaction terms in Eq. (4.21) is optimized at the expense of the kinetic energy. The point $J=2 t, n=0$ is possibly singular and $K_{\rho}$ there may depend on the order of the limits.

The phase diagram and the Luttinger liquid correlations of the $t-J$-model have also been established from variational wave functions [131, 132, 133]. This result is particularly noteworthy because these functions can be generalized into higher dimensions where exact solutions generally are not possible and numerical studies are severely limited by finite size effects (Section 6.2). Recall that, on a technical level, a major problem in treating the $t-J$-model with analytical methods, is the implementation of the constraint of excluded double occupancy. This constraint is implemented, however, in a variational wave function due to Gutzwiller 134

$$
\left|\Psi_{G}\right\rangle=\prod_{i}\left(1-n_{i \uparrow} n_{i \downarrow}\right)|F S\rangle \quad \text { with }|F S\rangle=\prod_{|k|<k_{F}} c_{k \uparrow}^{\dagger} c_{k \downarrow}^{\dagger}|0\rangle
$$

where $|F S\rangle$ is the filled Fermi sea and $|0\rangle$ the vacuum. This wave function yields rather good energies but the correlation hole between two particles it contains is too short. The momentum distribution has a sharp jump at $k_{F}$ but the spin correlations $($ at $n=1)$ are pretty close to the exact ones for a Heisenberg chain [135. To find a way to increase the range of correlations, notice the following. $\left|\Psi_{G}\right\rangle$ provides an exact solution to spin chains with an exchange integral falling off as $J \propto r^{-2}$ [136]. In the course of this solution, it has been shown that $\left|\Psi_{G}\right\rangle$ can be rewritten as

$$
\left|\Psi_{G}\right\rangle=\sum \operatorname{det}\left(e^{i q_{i} r_{j}}\right) \operatorname{det}\left(e^{i p_{i} r_{j}}\right) \prod_{j} S^{-}\left(r_{j}\right)|F M\rangle \propto \prod_{i<j} \sin ^{2}\left[\frac{\pi}{L}\left(r_{i}-r_{j}\right)\right],
$$

where $|F M\rangle$ denotes the fully (up)- spin-polarized ferromagnetic chain and $j$ labels the sites of the overturned spins. The size of the correlation hole can now be increased simply by increasing the power of the sines (Jastrow factor):

$$
\left|\psi_{\nu}\right\rangle=\prod_{\text {all } i<j}\left|\sin \left[\frac{\pi}{L}\left(r_{i}-r_{j}\right)\right]\right|^{\nu}\left|\psi_{G}\right\rangle
$$

where the notation under the product sign emphaised that in this product, the positions enter irrespective of the particles' spin direction. $\left(\left|\Psi_{\nu}\right\rangle\right.$ is also related to the quantum Hall effect as will be seen in Section [.3.). This can be seen quite explicitly from $\left|\psi_{\nu}\right|^{2}=$ 
$\prod_{i<j} \exp \left(-V_{i j}\right)$ with $V_{i j} \propto-\nu \ln \left|z_{i}-z_{j}\right|$ and $z_{i}=\exp \left(2 \pi i r_{i} / L\right)$, which represents the partition function of hard core objects with a logarithmic interaction [133]. It therefore can serve as a natural starting point for a variational treatment of the $t-J$-model. Correlation functions show power-law behaviour compatible with the Luttinger liquid form, Section 3.3, whose exponents now depend on the optimal value of $\nu$ which is obtained from variational Monte Carlo simulations. One can establish an explicit relation

$$
K_{\rho}=\frac{1}{2 \nu+1}
$$

to the Luttinger exponent $K_{\rho}$, and the numerical data are in good agreement. (4.53) can be derived either by finding a solvable model whose ground state is given by $\left|\psi_{\nu}\right\rangle . K_{\rho}$ can then be extracted from the spectrum of low-lying states exactly as for the Hubbard model above [137]. Another possibility is to computed explicitly the momentum distribution and then identify the exponent to Eq. (3.85) [138]. Finally, by applying increasing powers of the Hamiltonian to $\left|\Psi_{\nu}\right\rangle$, one can obtain increasingly accurate approximations to the exact ground state (provided that it is not orthogonal to the trial state $\left|\Psi_{\nu}\right\rangle$ ), and this method has allowed to uncover evidence for the formation of a spin gap in a region of very low carrier density and large $J / t$ close to the phase separation instability. In this limit, the system is a singlet superconductor. The resulting phase diagram is given in Figure 4.6, where "attractive Luttinger" stands for dominant TS and "repulsive Luttinger" implies dominant SDW correlations in a Luttinger liquid. The $t-J$-model can be generalized to include a $J \propto r^{-2}$ exchange, and its solutions are quite close to the Gutzwiller-Jastrow form discussed above.

One can also formulate Hubbard- and $t-J$-type models with long-range hopping which, in the limit of half-filled band, reduce to the Haldane-Shastry spin chain [99]. These models are exactly solvable but the solution in general is not Jastrow form. Away from half-filling, they have Luttinger liquid low-energy physics. One important element of these models is chirality, i.e. the hopping term must be constructed in such a way that the electronic dispersion contains only a single linear branch, corresponding to right-(or left-) moving particles alone. In this situation, the only allowed effective interaction of the electrons is of $g_{4}$-type, Eq. (3.4), while $g_{1}=g_{2}=g_{3}=0$. This still allows for charge-spin separation because $g_{4 \perp} \neq 0$ but the renormalized effective coupling constant $K_{\rho}=1$, the value for free fermions. This is an interesting situation because all $q$ - or $\omega$-dependent correlation functions will be indistinguishable from a Fermi liquid, still there are no quasiparticle excitations. From the discussion in Sec. 3.3 one would conclude, e.g., that the momentum distribution of such a model is a step function $n(k)=\Theta\left(k_{F}-k\right)$ but the spectral function $\rho(q, \omega)$ is purely incoherent with spectral weight between $v_{\sigma} q$ and $v_{\rho} q$ and square-root singularities at these frequencies.

At half-filling, the model exhibits a metal-insulator transition for $U \geq 2 \pi t$, as borne out by a jump in the chemical potential at $n=1$. How is this possible if Umklapp processes are forbidden? As $U \rightarrow 2 \pi t$ from below, the charge velocity diverges, corresponding to a divergence of $g_{4}$ ! At the same time, the compressibility goes to zero and the Drude weight of the conductivity also diverges. This is due to the dispersion exhibiting a jump 
discontinuity at the Brillouin zone boundary [99]. This is pretty opposite to the standard scenario, where relevant Umklapp processes generate the Mott-Hubbard transition, the charge velocity vanishes, and the Drude weight has a finite jump (Sec. 5.2). Here, the compressibility diverges and the Drude weight vanishes. The properties of the model in the charge sector therefore are peculiar and strongly affected by the pathological dispersion. The spin fluctuations, on the other hand, are more normal with a strong peak in $\chi(T)$ at $T \sim J=4 t^{2} / U$, and the instantaneous spin-spin correlation function has a logarithmic divergence at $q=\pi$, corresponding to $1 / r$-decay in space, as for the half-filled Hubbard model.

A further example for application of these methods, especially Eq. (3.62), is provided by the extended Hubbard model, Eq. (4.19). At half-filling, this model has been studied by many methods both analytical and numerical [50, 139]. This model possesses a rich phase diagram but, due to the importance of Umklapp scattering here, the system is insulating when the interactions are repulsive [50, 139]. Correlation functions and the identification of the phases as Luttinger liquids (eventually only in a single channel) have been studied using renormalization group [50]. The physics away from half-filling is also interesting. For $U \rightarrow \infty$, we recover spinless fermions, and the model can be mapped onto an anisotropic Heisenberg chain which again can be solved by Bethe Ansatz, so that the correlation exponents can be deduced [140, 141]. For finite $U$, Mila, Zotos, and Penc [142, 143] used numerical diagonalization combined with Eq. (3.62) to evaluate the phase diagram and correlation functions of the quarter-filled band. The phase diagram together with lines of constant $K_{\rho}$ in the positive $U, V$-region is given in Figure 4.7. On a technical level, this study is noteworthy because it is one of the few instances where the three velocities $v_{N \rho}, v_{\rho}$, and $v_{J \rho}$ have been determined explicitly and their consistency with the Luttinger liquid relations has been verified. For $v_{J \rho}$, one can use the relation of the Drude weight of the conductivity (precisely $2 v_{J \rho}$ ) to the dependence of the ground state energy on an external flux 43]

$$
v_{J \rho}=\left.\frac{\pi}{2 L} \frac{\partial^{2} E_{0}(\phi)}{\partial \phi^{2}}\right|_{\phi=0} .
$$

At weak coupling, the system is a Luttinger liquid, and its correlations are described by a parameter $K_{\rho}$, indicated in Fig. 4.7 as dashed lines. This parameter can now become smaller than $1 / 2$, the $U \rightarrow \infty$-limit of the Hubbard model. Due to the relevance of Umklapp scattering terms which have a scaling dimension of $2-8 K_{\rho}$, there is a lower limit $K_{\rho}=1 / 4$ in the metallic phase. Beyond, the system goes insulating, and $K_{\rho}$ discontinuously jumps to zero. The dominant correlations at weak-coupling are SDW but $2 k_{F}$-CDWs are only logarithmically weaker. For $K_{\rho}<1 / 3$, however, the divergence of the $4 k_{F}$-CDW correlations becomes stronger than the SDW one, indicating gradual charge localization on alternating sites. In the insulating phase, this charge modulation is long-range ordered, and the system can be viewed as a Wigner crystal. Fourth-order processes in $t$ still give an antiferromagnetic exchange interaction between occupied sites, and a $2 k_{F^{-}}$SDW consequently is superposed on the $4 k_{F^{-}}$CDW. As one goes to larger $V$ (unphysical if one thinks in terms of electron-electron interaction alone but conceivable if 
on-site phonons are included), the effective correlations get weaker, and $K_{\rho}$ can become even larger than unity. If the system is still a Luttinger liquid in this range, it would be dominated by triplet superconducting correlations before giving way to phase separation. (The caveat is important, since a conclusive study of the properties of the spectrum of the Hamiltonian on which the derivation of the Luttinger parameters is based, was not possible [142, 143], and Mila et al. conjecture about a two-fluid picture where a Luttinger liquid would coexist with a liquid of local singlet pairs.) A more complete diagram, including attractive interactions is also available [143]. Here, one can find $2 k_{F}$-CDWs, SS when a spin gap opens, another TS when there is no spin gap, and a phase separation regime when all interactions are attractive, as in the half-filled band (commensurability is unimportant at this point). Most of these results agree with a similar study by Sano and Ono [144] who, however, find evidence for a spin gap in the large- $V$-small- $U$ region, and who therefore would favour an SS phase preceding phase separation. Moreover, these authors extend these results to a third-filled band $(n=2 / 3)$ where similar results obtain except for strong repulsion. Here, no evidence for a transition to an insulating phase is found. While the general absence of such a transition is somewhat surprising, the Umklapp operators which could mediate such a transition are less relevant than at quarter-filling, and the transition may have escaped detection because is expected to occur at stronger coupling. Notice that for a third-filled band, $2 k_{F}=4 k_{F}=\pi / 3 a(\bmod 2 \pi / a)$, and that the Umklapp operator couples charges and spins (Section 5.2). One thus predicts the opening of a charge gap to be accompanied by opening of a spin gap. The competition of SDW and TS at positive $U$ and negative $V$, a situation that might be generated in two-band models, has been studied in detail by Kuroki et al. who, however, find somewhat poor agreement with renormalization group predictions even at weak coupling [145].

At general bandfilling $n$, the mapping onto a Heisenberg chain produces a finite magnetic field [140, 141]. Still, one can derive Luttinger parameters, and $K_{\rho}$ now can become as small as $1 / 8$ [49, allowing $\alpha$ to increase up to about 1.5. This confirms the earlier statement that the correlation exponents are strongly sensitive to the interaction range. A finite range is required to produce really strong correlations from strong interactions. This is exemplified most dramatically by considering a long-range Coulomb potential $V(r) \approx 1 / r$ [90]. In a continuum system, the charge fluctuations no longer have a linear spectrum at low $q$ but rather go as $\omega_{\rho}(q) \approx \sqrt{q^{2} \ln q}$, so that formally $v_{\rho}=0$. The spin fluctuations behave normal. The logarithmic low-energy spectrum also gives a peculiar dependence to the correlation functions involving density operators. The density-density correlations decay as

$$
\langle\rho(x) \rho(0)\rangle=A_{1} \cos \left(2 k_{F} x\right) \frac{\exp (-c \sqrt{\ln x})}{x}+A_{2} \cos \left(4 k_{F} x\right) \exp (-4 c \sqrt{\ln x})
$$

i.e. slower than any power of $x$ in its $4 k_{F}$-component. Comparing to (3.92) and (3.93), one formally would have $K_{\rho}=0$. The Green function behaves as $G(x) \approx \exp \left(i k_{F} x-c \ln ^{-3 / 2} x\right)$ and decays faster than any power of $x$ (formally $\alpha \rightarrow \infty$ ). The system is at the edge of Wigner crystallization. It retains some marginal Luttinger liquid character because the quantum version of the Mermin-Wagner theorem [146, 147] forbids a real phase transition 
into a long-range ordered 1D crystal.

On the lattice, a systematic investigation of the effects of band-filling on the structure of the ground state and thus the dominant CDW instability can be performed in the strong coupling limit under quite general conditions of convexity for a long-range density-density interaction 89, 148. In particular, from the minimization of the electronic interaction energy (i.e. in the atomic limit) one finds a series of generalized Wigner lattices as the band-filling $n$ is varied. For $n=1 / m$, one has every $m$-th site singly occupied with $m-1$ empty sites in between. For $n=2 /(2 m+1)$, the singly occupied sites are separated alternatingly by $m$ and $m-1$ empty sites. Other configurations can be constructed with a simple algorithm 89, 148]. As one dopes the system away from these rational bandfillings, one introduces solitons with fractional charge $q= \pm \rho e$ into the ground states. These particles are mobile if the hopping integral $t$ is finite, and they will experience an effective interaction. They will form again a Luttinger liquid, and their effective velocities $v_{\nu}$ and correlation exponent $K_{\nu}$ can be calculated as a function of the filling factor (the analysis is practical only for infinite interactions) [112].

\subsection{Electron-phonon interaction and impurity scat- tering}

How stable is the Luttinger liquid with respect to electron-phonon coupling?

There are several models describing different aspects of this interaction. Coupling of electrons to acoustic phonons is modelled by the Hamiltonian

$$
H_{\mathrm{SSH}}=-\sum_{i, s}\left(t_{0}-\alpha_{S S H}\left[u_{i+1}-u_{i}\right]\right)\left(c_{i+1, s}^{\dagger} c_{i, s}+\text { H.c. }\right)+\sum_{i}\left(\frac{P_{i}^{2}}{2 M}+\frac{K}{2}\left[u_{i+1}-u_{i}\right]^{2}\right)
$$

The electron-phonon coupling arises from the first-order modulation of the hopping integral by the relative displacements $u_{i+1}-u_{i}$ of two neighbouring sites. $K$ is the spring constant, $P_{i}$ the momentum operator, and $M$ the ion mass. Electron-electron interactions can be added if required. This model has been proposed to describe the essential physics of conducting polymers, and polyacetylene in particular, by $\mathrm{Su}$, Schrieffer, and Heeger ( $\mathrm{SSH}$ ) and most often has been studied close to half-filling [149, 150.

Electrons may also be coupled to intramolecular vibrations (optical phonons) which modulate the energy levels $\varepsilon_{i}$ of the lattice sites

$$
H_{\mathrm{Hol}}=-t_{0} \sum_{i, s}\left(c_{i+1, s}^{\dagger} c_{i, s}+\text { H.c. }\right)+\sum_{i}\left(\frac{P_{i}^{2}}{2 M_{r}}+\frac{f}{2} Q_{i}^{2}\right)+g \sum_{i} Q_{i} n_{i}
$$

This model is due to Holstein [151] and has played a central role in the polaron problem [152]. Here, the phonons are dispersionless, the spring constant is called $f$, and $g$ is the first order coupling of a molecular energy level to a vibrational coordinate $Q$ with an associated reduced mass $M_{r}$. Another model somewhat intermediate between the SSH and Holstein models, where the electrons couple to the librational motion of rings in a polymer chain, 
has been introduced and discussed recently [153. It combines dispersionless phonons with coupling to the hopping integral $t$. Phonon dispersion and different structures of the coupling terms lead to important differences in the physics of these models. Being not central to the subject of this article, we shall not detail them here but rather emphasize the common features of phonon-coupled Luttinger liquids.

Mean-field theory, the crudest form of an adiabatic approximation, does not lead to Luttinger liquid behaviour [5]. Rather, a gap opens and CDW long-range order obtains. Compatibility with the Mermin-Wagner theorem [146, 147] for incommensurate systems is reestablished by the Goldstone mode, the sliding of the CDW, but this only gives a gapless charge density excitation. The spins remain gapped, and the generic physics of such models is discussed further in Section 5.1. For genuine Luttinger liquid behaviour, one must therefore go beyond phonon mean-field theory and/or include electron-electron interaction. Due to the importance of electron-phonon backscattering in 1D [5], we give a brief discussion using renormalization group, directly extending Section 4.3 to include electron-phonon interaction.

The generic electron-phonon coupling Hamiltonian has the boson representation [154

$$
\begin{aligned}
H & =H_{1}^{e-p}+H_{2}^{e-p} \\
H_{1}^{e-p} & =\frac{\gamma_{1}}{\pi \alpha} \int d x\left\{\exp \left[\sqrt{2} i \Phi_{\rho}(x)\right] \cos \left[\sqrt{2} \Phi_{\sigma}(x)\right] \varphi_{2 k_{F}}(x)+\text { H.c. }\right\} \\
H_{2}^{e-p} & =\frac{\gamma_{2}}{\sqrt{L}} \int d x\left[\rho_{+}(x)+\rho_{-}(x)\right] \varphi_{0}(x)
\end{aligned}
$$

$H_{1,2}^{e-p}$ describe electron-phonon backward and forward scattering. $\varphi_{2 k_{F}}(x)$ is the $2 k_{F^{-}}$ component of the displacement field $Q_{i}$ or $u_{i+1}-u_{i}$, scaled by $\sqrt{M}$, and $\varphi_{0}(x)$ the $q \approx 0$ component. $\varphi_{0}(x)$ is a real field, but $\varphi_{2 k_{F}}(x)$ is complex because the scattering processes with $\pm 2 k_{F} \neq \pi / a$ are physically different. The coupling constants are

$$
\gamma_{1}^{(\mathrm{SSH})}=4 i \alpha_{S S H} \sin \left(k_{F} a\right), \quad \gamma_{2}^{(\mathrm{SSH})}=0 ; \quad \gamma_{1}^{(\mathrm{Hol})}=\gamma_{2}^{(\mathrm{Hol})}=g
$$

The vanishing of $\gamma_{2}$ for acoustic phonons only holds at $q=0$ and is a consequence of the linear dispersion in the centre of the Brillouin zone. The influence of the finite- $q$ contribution is of order $\left(v_{s} / v_{\rho}\right)^{2}$ where $v_{s}$ is the sound velocity of the phonons, and can be neglected in most realistic situations [155]. This may perhaps not be permitted close to a Mott transition where $v_{\rho} \ll v_{F}$ and may become comparable to $v_{s}$ [156.

Electron-phonon forward scattering in a Luttinger model can be diagonalized exactly [155]. Equivalent results are obtained by including it into the renormalization group. First, we integrate out the phonons to generate effective, retarded electron-electron interactions (in imaginary time formalism)

$$
\begin{aligned}
& H_{1}^{\mathrm{eff}}\left(\tau-\tau^{\prime}\right)= 2\left(\frac{\left|\gamma_{1}\right|}{2 \pi \alpha}\right)^{2} \int d x \int d x^{\prime} D_{0}\left(x-x, \tau-\tau^{\prime}\right) \exp \left(\sqrt{2} i\left[\Phi_{\rho}(x, \tau)-\Phi_{\rho}\left(x^{\prime}, \tau^{\prime}\right)\right]\right) \\
& \times \sum_{r} \cos \left(\sqrt{2}\left[\Phi_{\sigma}(x, \tau)+r \Phi_{\sigma}\left(x^{\prime}, \tau^{\prime}\right)\right]\right)+\text { H.c. } \\
& H_{2}^{\mathrm{eff}}\left(\tau-\tau^{\prime}\right)=\gamma_{2}^{2} \int d x \int d x^{\prime} \sum_{r, r^{\prime}} \rho_{r}(x, \tau) D_{0}\left(x-x^{\prime}, \tau-\tau^{\prime}\right) \rho_{r^{\prime}}\left(x^{\prime}, \tau^{\prime}\right)
\end{aligned}
$$


with the bare phonon propagator

$$
D_{0}(x, \tau)=\frac{1}{2 \omega_{p h}} \delta(x) \exp \left(-\omega_{p h}|\tau|\right) \quad .
$$

When deriving scaling equations for the effective coupling constants, $\alpha$ is interpreted as a cutoff which is also extended to the $\tau$-direction (Section 4.3). In order not to loose the short-time contributions which are important at high phonon frequencies $\omega_{p h}$, one must integrate the effective retarded interactions between $\tau-\tau^{\prime}=0$ and $\alpha / v_{F}$, giving effective instantaneous interactions, plus (4.62) and (4.63) with a cutoff $\alpha / v_{F}$ in $\tau$ [157. The instantaneous interactions are added to the electronic terms, and the retarded pieces are included into the renormalization group. Following Section 4.3, we obtain the following set of scaling equations [154, 157]

$$
\begin{aligned}
\frac{d K_{\rho}^{-1}}{d \ell} & =\frac{1}{2} \frac{v_{\rho}}{v_{\sigma}}\left(Y_{1}^{(p h)}-Y_{2}^{(p h)}\right) \mathcal{D}(\ell), \\
\frac{d K_{\sigma}^{-1}}{d \ell} & =\frac{1}{2}\left(Y_{\sigma}^{2}+Y_{1}^{(p h)} \mathcal{D}(\ell)\right) \\
\frac{d Y_{\sigma}}{d \ell} & =Y_{\sigma}\left(2-2 K_{\sigma}\right)-Y_{1}^{(p h)} \mathcal{D}(\ell) \\
\frac{d Y_{1}^{(p h)}}{d \ell} & =Y_{1}^{(p h)}\left(3-K_{\rho}-K_{\sigma}-Y_{\sigma}\right) \\
\frac{d Y_{2}^{(p h)}}{d \ell} & =Y_{2}^{(p h)}, \\
\frac{d v_{\nu}}{d \ell} & =\frac{1}{2} v_{\nu} K_{\nu}\left(Y_{1}^{(p h)}-Y_{2}^{(p h)}\right) \mathcal{D}(\ell) .
\end{aligned}
$$

The abbreviations are

$$
Y_{\sigma}=\frac{g_{1 \perp}}{\pi v_{\sigma}}, \quad Y_{1}^{(p h)}=\frac{\left|\gamma_{1}\right|^{2}}{\pi v_{\sigma} \omega_{p h}^{2}}, \quad \mathcal{D}=\frac{\alpha_{0} \omega_{p h}}{v_{\sigma}} \exp \left(-\frac{\alpha(\ell) \omega_{p h}}{v_{\sigma}}\right) .
$$

$Y_{2}^{(p h)}=Y_{1}^{(p h)}$ for dispersionless modes, and zero (for the present purposes) for acoustic modes.

A few important points are immediately apparent. (i) The phonon frequency $\omega_{p h}$, through $\mathcal{D}(\ell)$, is the decisive quantity controlling the interplay of repulsive electronelectron and attractive electron-phonon interactions. At a scale $\ell_{p h}=\ln \left(E_{F} / \omega_{p h}\right)$, all retardation effects are scaled out, and the model behaves (and eventually continues to renormalize) as effectively instantaneous. The influence of electron-phonon interaction is the stronger the lower $\omega_{p h}$. (ii) The charge degrees of freedom remain gapless for any nonvanishing phonon frequency. While $K_{\rho}$ rather strongly decreases for acoustic phonons, its sense of renormalization for dispersionless modes depends on the relative importance of forward and backward scattering. The intial $K_{\rho}(\ell=0)$ contains a contribution from the short-time part of $D_{0}$, as discussed above. (iii) A gap may open in the spin fluctuations, and in fact does so for low enough phonon frequency and / or high enough electron-phonon coupling. The system then is no longer a Luttinger liquid and its physics 
will be discussed in more detail in Section 5.1. Here SS or CDW correlations dominate, depending on $K_{\rho}$ [157, 158]. (iv) In the opposite limit of high phonon frequency and/or weak coupling, there is a Luttinger liquid regime. Depending on the renormalized $K_{\rho}$, we have SDW or TS correlations. The properties at low energies, below $\omega_{p h}$, are then given by the Luttinger liquid correlations (Section 3.3) with the fixed-point values $K_{\rho}^{\star}$ and $v_{\nu}^{\star}$. The scaling equations respecting spin-rotation invariance, $K_{\sigma}^{\star}=1$ is guaranteed for gapless spin fluctuations. At energies above $\omega_{p h}$, there will be deviations from the Luttinger liquid properties. An example, the Holstein contribution to the optical conductivity, involving phonon emission, will be discussed in the next section [159. Corrections to the spectral functions of a model with forward scattering only [155, 156], have also been evaluated [160]. (v) The velocities $v_{\nu}$ of the charge and spin fluctuations are renormalized by electron-phonon interaction. Consequently, this interaction has a pronounced influence on the thermodynamic properties such as specific heat, compressibility and susceptibility [159]. It is analogous to the enhancement of the effective mass, or the density of states at the Fermi level, familiar from higher-dimensional systems. In contrast to higher dimensions, the electron-phonon interaction couples charge and spin fluctuations and therefore strongly renormalizes the magnetic properties of the Luttinger liquid.

Renormalization group is also very useful to study the influence of impurity scattering on the low-energy properties of Luttinger liquids [161, 162]. The forward $(q \approx 0)$ and backward $\left(q \approx 2 k_{F}\right.$ ) electron-impurity scattering components can be represented by two Gaussian fields $\eta(x)$ and $\xi(x)$ with white noise correlations $P_{\xi}=\exp \left[-D_{\xi}^{-1} \int|\xi(x)|^{2} d x\right]$ and a similar expression for $P_{\eta}$ [163]. $D_{\eta(\xi)}=v_{F} / \tau_{\eta(\xi)}$ and $\tau$ is the scattering time. The interaction Hamiltonian is

$$
\begin{aligned}
H_{f} & =\sum_{r s} \int d x \eta(x) \Psi_{r s}^{\dagger}(x) \Psi_{r s}(x)=-\frac{\sqrt{2}}{\pi} \int d x \eta(x) \frac{\partial \Phi_{\rho}(x)}{\partial x} \\
H_{b} & =\sum_{s} \int d x\left[\xi(x) \Psi_{+s}^{\dagger}(x) \Psi_{-s}(x)+\xi^{\star}(x) \Psi_{-s}^{\dagger}(x) \Psi_{+s}(x)\right] \\
& =\frac{1}{\pi \alpha} \int d x\left\{\xi(x) e^{i\left[\sqrt{2} \Phi_{\rho}(x)+2 k_{F} x\right]} \cos \left[\sqrt{2} \Phi_{\sigma}(x)\right]+\text { H.c. }\right\}
\end{aligned}
$$

This Hamiltonian is of the same structure as the electron-phonon interaction (4.59) and (4.60) except that $\eta(x)$ and $\xi(x)$ are static fields while $\varphi_{0}(x)$ and $\varphi_{2 k_{F}}(x)$ posses dynamics. The renormalization group treatment therefore is parallel to the phonon problem up to two differences: (i) the "phonon frequency" $\omega_{p h}=0$ here to reflect the static nature of the impurity fields; (ii) for the same reason, forward scattering can be completely eliminated by simply shifting

$$
\Phi_{\rho}(x) \rightarrow \tilde{\Phi}_{\rho}(x)=\Phi_{\rho}(x)-\frac{\sqrt{2} K_{\rho}}{v_{\rho}} \int^{x} d z \eta(z)
$$

and completing the square. More importantly, if one uses the replica trick to treat backscattering, the resulting action only contains differences of $\Phi_{\rho}$-fields so that they are not affected by the shift (4.69). Also unaffected by this shift are the $\Pi_{\rho}(x)$ - and $\Theta_{\rho}$-fields because they are generated from $\Phi_{\rho}$ by time derivatives. This immediately implies that both the conductivity and the pairing fluctuations (SS and TS) are unaffected 
by electron-impurity forward scattering. The charge and spin density wave correlation functions, on the other hand will decay exponentially with distance

$$
R_{C D W, S D W}(x, t)=\left.e^{-D_{\eta}\left(\frac{K_{\rho}}{v_{\rho}}\right)^{2}|x|} R_{C D W, S D W}(x, t)\right|_{\eta \equiv 0}
$$

Decay with time is not affected.

Also the influence of electron-impurity backscattering $\xi(x)$ is dramatic. The renormalization group equations (4.65) can be taken over directly [161] with the phonon $\mathcal{D}$ dropped, $Y_{2}^{(p h)}=0$, and $Y_{1}^{(p h)}$ is replaced by a new

$$
\mathcal{D}=\frac{2 D_{\xi} \alpha}{\pi v_{\sigma}^{2}}\left(\frac{v_{\sigma}}{v_{\rho}}\right)^{K_{\rho}} .
$$

The scaling dimension of the impurity backscattering operator $\mathcal{D}$ is $3-K_{\rho}-K_{\sigma}^{\star}$ and determines its (ir)relevance in the limit $\mathcal{D}, Y_{\sigma} \rightarrow 0$ where mutual renormalization effects can be neglected [162]. $\mathcal{D}$ goes relevant except for $K_{\rho}>2$ for a SU(2)-invariant system $\left(K_{\sigma}^{\star}=1\right)$ and for $K_{\rho}>3$ for a spin-gapped system $\left(K_{\sigma}^{\star}=0\right)$ : disorder is always relevant except deep in the superconducting region, and more so for a triplet superconductor than for a singlet one. The fact that even weak disorder becomes relevant for weak superconducting correlations $\left(1<K_{\rho}<2\right)$ shows that Anderson's theorem 164 fails in 1D. A Luttinger liquid only occurs when the coupling constants of the pure system were such that it is strongly TS ( $K_{\rho}^{\star} \geq 2$ required), and TS correlations then continue to dominate. Disorder can also be irrelevant when a spin gap opens $\left(Y_{\sigma} \rightarrow-\infty\right)$ with SS correlations strongest, but very large $K_{\rho}^{\star} \geq 3$ is called for here. In all other cases, both $\mathcal{D}$ and $-Y_{\sigma}$ flow to infinity. Disorder is relevant, and localization occurs. Moreover, one always has a spin gap, and the physics then is best described as a CDW $\left(K_{\rho}^{\star}=0\right)$ pinned by impurities 165 (charge density glass). For strong enough repulsion between the electrons, one would however expect localization in the presence of antiferromagnetic correlations. Such a random antiferromagnet is, in fact, conjectured by Giamarchi and Schulz [161] and their failure to find it identified as an artefact of the development leading to the renormalization group equations. The main features of the RG equations can also be rationalized by realizing that the impurity backscattering operator linearly couples to the CDW-operator (3.89) while the other types of fluctuations (SDW, SS, TS) are only influenced in higher order. It is therefore clear, that the charge density glass phase descending from CDWs strongly extends in the phase diagram.

The influence of impurities and electron-phonon scattering on transport is a subject of the following section.

\subsection{Transport in Luttinger liquids}

\subsubsection{Electron-electron scattering}

In the presence of band curvature and in lattice models, the Hamiltonian does not commute with the current which no longer is proportional to the momentum, and nonvanishing 
conductivity at finite frequencies is possible. There are several processes contributing. The most obvious ones are Umklapp processes whereby $n$ electrons are transferred from one side of the Fermi surface to the other, carrying with them momentum of the order $\pm 2 n k_{F}$. These processes are possible at low energy only in commensurate systems where the Fermi wave vector has a rational relation to a reciprocal lattice vector $G$ : $k_{F}=(m / n) G$. Away from these commensurate band-fillings they involve states separated from the Fermi level by a finite energy gap $\Delta_{m / n}$ and will therefore contribute to the conductivity only at frequencies or temperatures above $\Delta_{m / n}$ 44, 45]. These issues will be discussed further in Section 5.2. Another contribution comes from band curvature. In the presence of interactions, band curvature will also renormalize the current operator [3, 45].

Band curvature in an incommensurate system adds a term (4.2) to the Luttinger Hamiltonian (3.1). The current operator is given by Eq. (3.67), and its commutator with the Hamiltonian for small $q$ reduces to

$$
\lim _{q \rightarrow 0}[H, j(q)]=\frac{\lambda}{48 m^{2} v_{F}}+\ldots
$$

Then, using Eq. (3.68), one obtains at $T=0$

$$
\sigma(\omega>0)=\frac{1}{8 \pi}\left(\frac{\lambda}{12 m^{2} v_{F}}\right)^{2} \frac{K_{\rho}-K_{\rho}^{-1}}{4 v_{\rho}^{3}} \omega^{3}+\ldots
$$

i.e. a universal (interaction-independent) $\omega^{3}$-law 45 . This result is essentially perturbative in the band-curvature. Both for $K_{\rho} \rightarrow 1$ (noninteracting electrons) and $\lambda / m^{2} \rightarrow 0$ [free electrons with $k^{2} / 2 m$-dispersion, i.e. Galilei invariance $(\lambda \rightarrow 0)$ ], or Lorentz invariance $(m \rightarrow \infty)$ ] the finite frequency contribution disappears, as it must according to Chapter 3. A direct calculation of the temperature dependence of the dc-conductivity is not possible, but Giamarchi and Millis [45] give arguments for a divergence faster than any power of $1 / T$ as $T \rightarrow 0$.

In these calculations, there is no mechanism for dissipation. Ogata and Anderson argue that special boundary conditions must be used in order to allow for dissipative effects [166]. Further neglecting vertex corrections, they find that the dc-resistivity and conductivity vary as

$$
\rho(T)=\frac{1}{\sigma(T)} \sim T^{1-2 \alpha}
$$

where $\alpha$ is the single-particle exponent. For $\alpha \ll 1$ as we have in the 1D Hubbard model the resistivity varies nearly linearly with temperature. This behaviour should be closer to real systems than the Hamiltonian-based calculations outlined before. The frequency dependence then is determined by a relaxation rate linear in $\omega$ :

$$
\sigma(\omega) \sim \frac{\omega^{2 \alpha}}{i \omega+\omega \tan \pi \alpha}
$$

That the optical conductivity in real materials could essentially probe the density of states and thus depend on powers of $\alpha$ had also been conjectured earlier [167. 
Finally, some information can also be obtained by other methods. Carmelo and Horsch [168 calculate the weight of the $\delta(\omega)$-peak in the conductivity of the 1D Hubbard model directly from the Bethe-Ansatz wave function and provide an interpretation from the Landau-Luttinger-liquid point-of-view (Section 4.7).

Obviously, while electron-electron scattering is one conduction-limiting mechanism, experiments often probe other influences: scattering off impurities and phonons. Much work has been done on the impurity problem, less on electron-phonon scattering, and we start with the former.

\subsubsection{Electron-impurity scattering}

We shall proceed in two steps: (i) a single (or double) impurity and (ii) a system containing a finite concentration of impurities.

If we consider a single impurity in a Luttinger liquid, it is convenient to compute the conductance $G$ rather than the conductivity $\sigma$ of the system. The conductance is defined on a sample of finite dimensions by $G=1 / R$ in terms of the resistance, and related macroscopically to the conductivity $\sigma=1 / \rho$ by $G=\sigma A / L$ where $A$ is the cross section (= 1 in our $1 \mathrm{D}$ problems) and $L$ the length of the system. Microscopically, $G$ can be computed via a Kubo formula [169]

$$
G=\lim _{\omega \rightarrow 0} \frac{1}{\hbar L \omega} \int_{0}^{L} d x \int d \tau e^{i \omega \tau}\left\langle T_{\tau} J(x \tau) J(00)\right\rangle
$$

For reference, one can evaluate this expression for the impurity-free Luttinger liquid and finds

$$
G=n K_{\rho} \frac{e^{2}}{h}
$$

where $h=2 \pi \hbar$ and $n$ is the number of channels ( $n=2$ for spin- $1 / 2$ electrons). This result has been found earlier by Apel and Rice [170]. Notice that in contrast to $\sigma, G$ is renormalized by the electron-electron interactions. At first sight, this is not surprising since the finite length breaks the translational invariance of the system which is the basis of the independence of $\sigma$ of electronic interactions. On the other hand, $G$ can also be defined for $L \rightarrow \infty$ and then gives a results different from the Drude weight in the conductivity. It thus appears that the limit $L \rightarrow \infty$ and the process of turning on the electron-electron interaction do not commute.

We now include a single or double impurity, mainly following Kane and Fisher 169]. Equivalent results have been obtained by Furusaki and Nagaosa [171]. For simplicity, we restrict ourselves to spinless fermions $\left(n=1, K_{\rho} \rightarrow K\right)$. There are two complementary starting points: a weak impurity where perturbation theory in the impurity potential works, and a strong impurity which can be viewed as two weakly connected semi-infinite Luttinger liquids. In the first case, the Hamiltonian for an impurity at $x=0$ is

$$
\delta H=\int d x V(x) \Psi^{\dagger}(x) \Psi(x)
$$

with $V(x)$ strongly peaked around $x=0$. The action for its dominant contribution, backscattering of $m$ electrons across the Fermi surface, i.e. transferring momentum 
$\pm 2 m k_{F}$, is

$$
\delta S \approx \sum_{m=-\infty}^{\infty} \frac{v_{m}}{2} \int d \tau e^{i 2 m \Phi(x=0, \tau)}
$$

$v_{m}$ is the Fourier transform of $V(x)$ at $2 m k_{F}$. Here, we have included the higher harmonics from $\Psi(x)$ which occur in the Luttinger liquid (Section 4.2). The weak link, on the other hand, can be modelled by a hopping Hamiltonian

$$
\delta H \approx-t\left[\Psi_{l}^{\dagger}(x=0) \Psi_{r}(x=0)+\text { H.c. }\right] .
$$

Now one traces over the degrees of freedom away from the impurity $(x \neq 0)$ to obtain an effective action for $x=0$ only. This can then be used to compute the conductance through the impurity. To this end, one derives renormalization group equations for $v_{m}$ or $t_{m}$

$$
\frac{d v_{m}}{d \ell}=\left(1-m^{2} K\right) v_{m}, \quad \frac{d t_{m}}{d \ell}=\left(1-\frac{m^{2}}{K}\right) t_{m} .
$$

For repulsive interactions $K<1$, the most relevant backscattering term $v_{1}$ increases under scaling, i.e. an initially weak impurity behaves effectively as a strong one. This qualitative conclusion is supported by the strong-coupling limit where $t_{1}$ (and all higher $\left.t_{m}\right)$ is irrelevant, i.e. the two Luttinger liquids are effectively isolated. The impurity thus produces total reflection for repulsive interactions. On the other hand, for attractive interactions, $K>1$, all $v_{m}$ are irrelevant and at least $t_{1}$ is relevant, i.e. the impurity allows for total transmission. At the fixed point, in the former case the resulting conductance is $G=0$, in the latter one has the ideal Luttinger liquid conductance $G=K e^{2} / h$.

When temperature $T$, frequency $\omega$, or voltage $V$ are finite, they provide an effective cutoff to the renormalization group flow and produce power-law corrections to the fixed point conductances. One finds [with $\Omega=\max (\omega, T, V)$ and $n=1$ for spinless fermions]

$$
G(\Omega)=\frac{e^{2}}{h}\left[K-\sum_{m=1}^{\infty} a_{m \Omega}\left|v_{m}\right|^{2} \Omega^{2\left(m^{2} K-1\right)}\right] .
$$

The expansion coefficients $a_{m \Omega}$ are nonuniversal but their ratios are universal. Power-laws similar to the second terms on the right-hand side may be derived for transport through a weak link [169, 171].

Including spin degrees of freedom, the physics becomes much richer. The Hamiltonian for scattering off an impurity now becomes

$$
\begin{aligned}
\delta H & =\sum_{s} \int d x V(x) \Psi_{s}^{\dagger}(x) \Psi_{s}(x) \\
\delta S & =\sum_{m_{\rho}, m_{\sigma}} v_{m_{\rho}, m_{\sigma}} \int d \tau \cos \left(\sqrt{2} m_{\rho} \Phi_{\rho}\right) \cos \left(\sqrt{2} m_{\sigma} \Phi_{\sigma}\right)
\end{aligned}
$$

and for a weak link connecting two semi-infinite spin-1/2 Luttinger liquids

$$
\begin{aligned}
\delta H & =-t \sum_{s}\left[\Psi_{r s}^{\dagger}(0) \Psi_{l s}(0)+\text { H.c. }\right] \\
\delta S & =\sum_{m_{\rho}, m_{\sigma}} t_{m_{\rho}, m_{\sigma}} \int d \tau \cos \left(\sqrt{2} m_{\rho} \Theta_{\rho}\right) \cos \left(\sqrt{2} m_{\sigma} \Theta_{\sigma}\right), m_{\rho}=m_{\sigma} \bmod 2 .
\end{aligned}
$$


Notice how the impurity couples charge and spin degrees of freedom. In addition to the (charge) conductance $G \equiv G_{\rho}$, Eq. (4.76), a spin conductance $G_{\sigma}=2 K_{\sigma} e^{2} / h$ can be defined. Then several phases are possible in principle, depending on the interactions $K_{\nu}$ : (i) the impurity can be irrelevant for charge and spin so that one recovers the perfect conductor $G_{\nu}=2 K_{\nu} e^{2} / h$; (ii) the impurity can be relevant in one channel only, i.e. one has a charge conductor and spin insulator $\left(G_{\rho}=2 K_{\rho} e^{2} / h\right.$ and $\left.G_{\sigma}=0\right)$ or vice versa; (iii) the impurity is relevant and one has a perfect insulator $G_{\nu}=0$. Treating the impurity potential or the weak link by renormalization group, one finds that the most relevant term is $2 k_{F}$-backscattering of an electron on the impurity. In the limit of small impurity potential, the renormalization equation for $v_{m_{\rho}, m_{\sigma}}$ is

$$
\frac{d v_{m_{\rho}, m_{\sigma}}}{d \ell}=\left(1-m_{\rho}^{2} \frac{K_{\rho}}{2}-m_{\sigma}^{2} \frac{K_{\sigma}}{2}\right) v_{m_{\rho}, m_{\sigma}}
$$

In the spin-symmetric case $\left(K_{\sigma}=1\right)$, the lowest term with $m_{\rho}=m_{\sigma}=1$ is relevant [case (iii)] for repulsive interactions $\left(K_{\rho}<1\right)$, marginal for free electrons $\left(K_{\rho}=1\right)$ and irrelevant $\left[\right.$ case (i)] for attractive interactions $\left(K_{\rho}>1\right)$, as in the spinless case. Also corrections to the fixed point conductances can be evaluated, and one finds expressions very similar to Eq. (4.82) from the spinless case. Case (ii) obtains when, for some reason, a potential component with $m_{\rho}>1$ much larger than $v_{1,1}$ and has to be incorporated first. Its principal effect is to fix the phase of $\Phi_{\rho}$ at the impurity to a preferred value. With respect to this phase-quenched situation, $v_{1,1}$ is irrelevant if $K_{\rho}<2$ and $K_{\sigma}>2$ in general, and if $K_{\sigma}>1 / 2$ for symmetric potentials. All indices $\rho \leftrightarrow \sigma$ if a potential component with $m_{\sigma}>1$ is large, and the criterion of symmetry of the scattering potential is replaced by spin symmetry of the barrier. There are also several fixed points at intermediate couplings where explicit calculations are possible [169.

For finite impurity strength, consistency requires to include the irrelevant electronic interactions into the renormalization group scheme. This applies to $g_{1 \perp}$ in the spin$1 / 2$ case. Matveev et al. 172 treat a Fermi gas plus perturbative interactions. $g_{1 \perp}$ renormalizes $K_{\sigma}$ which enters the conductance exponent. Translated into a conductance, one obtains logarithmic corrections to the power-laws characterizing the (electronic) fixedpoint properties. Matveev et al. also claim that the temperature dependence of the conductance changes to nonmonotonic for $g_{1}$ more repulsive than a critical strength. Electron-electron backscattering can be quenched by applying a magnetic field. This gives an interesting crossover behaviour to the conductance. At energy scales larger than $2 \mu_{B} B$ ( $\mu_{B}$ is the Bohr magneton), backscattering is present and the system behaves as a Luttinger liquid. Below $2 \mu_{B} B$, the external field blocks the backscattering contribution, and the scaling behaviour of spinless electrons applies. One then has to match both sets of equations at $\ell_{B}=\ln \left(2 \mu_{B} B\right)$. Matveev et al. also argue that backscattering can be restored by applying a finite bias $V$, and predict a cusp-singularity in the differential conductance at $V=2 \mu_{B} B / e$.

The analysis can be generalized to a situation with two impurities creating an island between two semi-infinite Luttinger liquids [169, 173]. By fine-tuning a parameter, e.g. the energy of the (noninteracting and spinless) electrons incident on the barrier, one 
finds resonances with perfect transmission at certain energies although they have a finite width even at $T=0$. As one turns on repulsive interactions, interesting changes take place. One still needs to tune the energy of the incident electrons in order to make the $2 k_{F}$-backscattering matrix element $v_{1}$ vanish. (i) Then, however, the resonances become infinitely sharp as $T \rightarrow 0$ - the interactions suppress all off-resonance conductance. (ii) The conductance exactly at resonance depends on the strength of the electron-electron repulsion and, eventually, on the impurity strength at higher multiples of $2 k_{F}$. In particular, for $1>K>1 / 2$ (and also for $1 / 2>K>1 / 4$ provided that $v_{2}$ is small enough), one recovers the full Luttinger liquid conductance $K e^{2} / h$ at resonance with zero conductance off resonance. Only for $K<1 / 4$, or $K<1 / 2$ and $v_{2}$ large enough, is zero conductance obtained. Of course, for attractive interactions, the barriers become irrelevant, there are no resonances and one recovers the full Luttinger liquid conductance without fine-tuning.

Including spin, there are important differences, as can be seen easily in the limit of very strong barriers [169]. The charge on the island now is discrete; if it is odd, there will be a spin degeneracy as for a local magnetic moment. This is reminiscent of the Kondo effect, where a magnetic impurity $(s=1 / 2$ in the simplest case) is embedded in a Fermi sea of electrons. Resonant transmission through the island is again possible upon fine-tuning one (or several) parameters.

Generically, there are two types of resonances which can be achieved tuning one parameter only. The Kondo resonance is the generalization of the spinless fermion resonance discussed above. Suppose that we have tuned the $2 k_{F}$-backscattering term (4.83) to zero. Transmission will then depend on whether the next-to-leading terms $\left(m_{\rho}\right.$ or $\left.m_{\sigma}=2\right)$ are relevant or not. For $K_{\sigma}=1, v_{1,2}$ is harmless, and $v_{2,1}$ blows up only if $K_{\rho}<1 / 2$. This implies that, for $1>K_{\rho}>1 / 2$, both spin and charge are perfectly transmitted on resonance (although a single barrier would be totally reflecting), but for $K_{\rho}<1 / 2$, charge is totally reflected while spin is transmitted on resonance. Off resonance, there is no conductance, as for spinless fermions. Of course, for attractive interactions $\left(K_{\rho}>1\right)$ the barriers are irrelevant altogether. Allowing for $K_{\sigma} \neq 1$, one can also find a phase which transmits charge and reflects spin.

Another resonance is possible when both $v_{1,1}$ and $v_{2,1}$ become relevant, i.e. $K_{\rho}<1 / 2$. For a symmetric potential and $v_{1,1}$ only, one obtains charge and spin insulating barriers. For $v_{2,1}$ only, the barriers are charge insulating and spin conducting. As a function of $v_{1,1} / v_{2,1}$, one will have a charge resonance with finite conductance in between two charge insulating phases. In a case with broken spin-rotation invariance, this intermediate fixed point is accessible perturbatively, and its properties can be computed in some detail [169]. Its main interest lies in its finite charge conductance, because the generic Kondo resonance in this regime has $G_{\rho}=0$ and is thus difficult to observe.

More relevant experimentally is the shape of the resonance (an $I-V$-characteristic, for example) as a control parameter $\delta$ (e.g. the gate voltage on the island) is tuned through the resonance. Perfect resonance is achieved when the renormalized potential $v^{\star}=0$. Off resonance, the conductance will be determined by the growth of $v$ as it flows away from the fixed point $v^{\star}=0$. According to Eq. (4.85), $v_{1,1}$ grows with an exponent $\lambda=1-K_{\rho} / 2-K_{\sigma} / 2$ (resp. $1-K$ for spinless fermions). Close to the critical point, there 
will be a vanishing frequency scale $\Omega \sim \delta^{1 / \lambda}$. Here, one then expects the conductance depend in a universal way on the ratio $\Omega / T$

$$
G(T, \delta) \sim \tilde{G}\left(c \delta / T^{\lambda}\right)
$$

where $\tilde{G}$ is a universal scaling function. For small argument, one can expand $\tilde{G}$ to second order about the fixed-point value $G^{\star}$. For large arguments, i.e. far from the critical point, one can match onto the conductance at finite temperature in the single-strong-impurity limit. In this way, one finds

$$
\tilde{G}(X) \sim X^{-2 / K_{\rho}}
$$

(drop the index $\rho$ for fermions without spin). Only for a noninteracting system is the line shape Lorentzian. If interactions are present $\left(K_{\rho} \neq 1\right)$, the tails of the resonance line will be suppressed (repulsion) or enhanced (attraction). For spinless fermions, these scaling arguments can be backed up by an exact nonperturbative calculation for a special value of the coupling constant $K=1 / 2$. Moreover, for another value $K=1 / 3$, relevant for the Luttinger liquid description of the fractional quantum Hall effect at $\nu=1 / 3$ (where $\nu$ is the Landau level filling factor) 174 (Section 6.3), quantum Monte Carlo simulations give excellent agreement with the scaling prediction (4.87) and, in addition, provide the complete scaling function $\tilde{G}(X)$ for all $X$ [175.

A particularly detailed discussion of the line shapes is given by Furusaki and Nagaosa either for the tail region of a strong resonance or in the limit of strong barriers (weak link) 173. They showed that, only for strong electron-electron interaction (no matter what its sign), both width and height of the peak vary monotonically with temperature. Nonmonotonic behaviour in one of these quantities is observed for weaker interactions $1 / 2<K<2$ : for repulsive interactions, the peak height passes through a minimum between its high-temperature value and the low- $T$ fixed point conductance $K e^{2} / h$, while for moderate attraction, the peak width passes through a minimum. The crossover in both cases is determined by the ratio of temperature to the island quantization energy $\delta \epsilon \sim v_{F} / R$.

These results apply to short-range i.e. well-screened interactions. This is presumably relevant for quasi-1D organic metals but a doubtful hypothesis for semiconductor quantum wires where the electron density both in the wire and in its environment is low. Then, the long-range nature of the Coulomb interactions has to be taken into account 90. There are two essential modifications [176]: (i) the conductance of even the pure Luttinger liquid is length $(L)$ dependent as

$$
G(L)=\frac{3 e^{2} \nu}{h \sqrt{R_{\perp} / L}}
$$

where $R_{\perp}$ is the transverse extension of the quantum wire, and $\nu=(2 / 3) \sqrt{\left(1+g_{1}\right) \pi / 4 \zeta}$ (with $g_{1}$ the $2 k_{F}$-component of the Coulomb potential, $\zeta=e^{2} / \kappa v_{F}$, and $\kappa$ a dielectric constant simulating the environment). The length-dependence of $G$ apparently indicates a vanishing of the Drude weight in the conductivity of the infinite system. This is interpreted as being due to the vanishing compressibility of this system with long-range interactions, 
Eqs. (3.62) and (3.66) with $K_{\rho} \rightarrow 0$ [Section 4.4, after Eq. 4.55)]. (ii) The conductance through an impurity vanishes faster than any power of $T$ or $V$ (replace $T \rightarrow e V$ below)

$$
G(T) \sim \exp \left[-\nu \ln ^{3 / 2}\left(T_{0} / T\right)\right]
$$

This is very much reminiscent of threshold behaviour.

Essentially in disagreement with this work, an earlier calculation [177] finds a powerlaw variation of the resistivity with temperature by treating the scattering with a $f_{\text {- }}$ nite density of impurities in Born approximation. Using the same method, Ogata and Fukuyama later studied the crossover taking place as a function of system size and temperature, between regimes of quantized conductance implying infinite dc-conductivity, and finite conductivity 178 . They show that, for small $L$, one better thinks in terms of a conductance $G=2 K_{\rho} e^{2} / h$ (including spin) while beyond a given system size (determined by temperature and the elastic mean free path), the conductance crosses over to a $1 / L$-behaviour which implies finite dc-conductivity. While details of their prediction may depend on computational procedures, the existence of such a crossover seems quite plausible both for short and long range interactions.

We now pass on to the problem of many randomly positioned impurities. Here, the interference of the scattered electrons becomes important and can lead to localization [179]. In a noninteracting 1D electron gas in the presence of disorder, all states will be localized [180]. This need no longer be so if electron-electron interaction is turned on, and we have given a general discussion of their mutual renormalization in Section 4.5. Here we sketch their influence on transport.

One important feature is already apparent when comparing the renormalization group flow of a single impurity [4.85) with $\left.m_{\rho}=m_{\sigma}=1\right]$ and of many impurities (Section 4.5) in a Luttinger liquid. For the noninteracting system, the single impurity is marginal, i.e. does not change its conductance, while a finite concentration of impurities is strongly relevant and leads to localization (the interacting system follows the same logic). The difference is due to quantum interference, i.e. an electron multiply scattered off impurities interferes with its time reversed shadow. This leads to an effective backward scattering of the electron and enhanced localization. The process is absent for a single impurity.

The renormalization group equations allow to determine, in some cases, the localization length $L_{l o c}$ and temperature $T_{l o c}=v_{F} / L_{l o c}$ beyond/below which localization takes place. For small disorder $\mathcal{D} \rightarrow 0$, and close to the critical surface where the disorder is marginal, one finds respectively

$$
L_{l o c} \sim \mathcal{D}^{-1 /\left(3-K_{\rho}-K_{\sigma}^{\star}\right)} \quad, \quad L_{l o c} \sim \exp \left(\frac{K_{\rho}-2}{\mathcal{D}-\left(K_{\rho}-2\right) Y_{\sigma}}\right)
$$

In the spin-gap phases, these equations change [put $K_{\sigma}^{\star}=0$ and replace $\exp (\ldots)$ by $\exp \left\{1 / \sqrt{9 \mathcal{D}-\left(K_{\rho}-3\right)^{2}}\right\}$ in $\left.(4.90)\right]$.

To determine the temperature-dependent conductivity $\sigma(T)$, we observe that thermal fluctuations will break coherence at a length scale $\xi_{T}=v_{F} / T$, and renormalization will stop there. The conductivity can then be calculated in Born approximation. In the 
delocalized phase, one obtains $\sigma \sim T^{-1-\gamma}$ with $\gamma=K_{\rho}^{\star}-2$ in the TS region and $\gamma=K_{\rho}^{\star}-3$ in the SS region, and $K_{\rho}^{\star}$ is the renormalization group fixed point value. In the localized region, $T_{l o c}$ sets a crossover scale: for $T>T_{l o c}$, conductivity first increases with decreasing temperature, and only for $T<T_{l o c}$ the quantum interference leading to localization and a decreasing $\sigma(T)$ sets in. Above (below) $T_{l o c}\left(L_{l o c}\right)$, quantum interference is unimportant and one has a diffusive regime (absent for noninteracting 1D electrons). Furusaki and Nagaosa have refined this picture by pointing out that there is another temperature scale $T_{\text {dis }}=v_{F} / k_{B} R>T_{\text {loc }}$ where $R$ is the mean impurity distance 171. For $T_{\text {dis }}>T>T_{\text {loc }}$, there is no localization (quantum interference) and the impurities behave as isolated. Electron-electron interactions are present however, and the conductivity of the system is governed by the scattering off individual impurities as considered in the beginning of this Section. $T_{d i s}$ necessarily exceeds $T_{l o c}$ because localization can take place only on length scales beyond the mean impurity distance.

\subsubsection{Electron-phonon scattering}

One may finally inquire about the influence of electron-phonon scattering on the conductivity of a Luttinger liquid. The renormalization group equations for the electron-phonon problem (4.65) are strongly controlled by $\omega_{p h} / E(\ell)$. For $\ell>\ln \left(E_{F} / \omega_{p h}\right)$, they reduce to a purely electronic problem with renormalized starting parameters. For an incommensurate system where there are no non-Luttinger interactions left in the charge channel, once all retardation effects have been renormalized away one expects to find a Drude peak with a renormalized weight $2 v_{\rho}^{\star} K_{\rho}^{\star}$ where $v_{\rho}$ and $K_{\rho}$ are obtained from (4.65). These parameters generically decrease under renormalization so that the conductivity is lowered by electron-phonon scattering. An exception occurs only in the high-phonon-frequency regime of models with significant forward scattering which do have dominant superconducting fluctuations [157]. At temperatures above the phonon frequency, the renormalization stop is determined by $T$, and one can take over the conductivity results from the impurity scattering problem.

Interesting effects occur in the optical conductivity $\sigma(\omega)$. In the presence of phonons, a new absorption process (Holstein absorption) is allowed: upon absorbing a photon ( $\omega$ finite, $q \approx 0)$, one creates a particle-hole pair $\left[\omega_{p-h}=\omega-\omega_{p h}(q)\right]$ and a phonon $\left[\omega_{p h}(q)\right]$ whose essential task is to take up the momentum imparted by the particle-hole pair. Such a process is possible, of course, only for $\omega>\omega_{p h}$, and the additional optical conductivity generated, has been computed in second order for a $2 k_{F^{-}}$phonon as 159

$$
\sigma_{h o l}(\omega) \sim \Theta\left(\omega-\omega_{2 k_{F}}\right)\left|\omega-\omega_{2 k_{F}}\right|^{1-\alpha_{C D W}}
$$

where $\alpha_{C D W}$ is the CDW correlation function exponent (3.92). In higher orders, one has to take account of the lattice softening induced by the electron-phonon coupling, and there will be Holstein conductivity for all $\omega>0$ varying again as a power-law for small $\omega$. Small momentum scattering, on the other hand, in inefficient in generating additional conductivity in the absence of band curvature. Physically, this is so because the particle and the hole generated travel with the same group velocity and therefore 
will recombine with probability one. In the presence of band-curvature, there is a finite forward contribution to the Holstein conductivity.

\subsection{The notion of a Landau-Luttinger liquid}

We have seen in Section 4.4.2 that the Bethe Ansatz solution of the Hubbard model (4.24) is generated from the distributions of two quantum numbers $\left\{I_{i}\right\}$ and $\left\{J_{\alpha}\right\}$ via the Lieb$\mathrm{Wu}$ equations (4.25) and (4.26). In the ground state, $\left\{I_{i}\right\}$ and $\left\{J_{\alpha}\right\}$ occupy consecutive integer or half-odd-integer values once and only once, so that the distribution functions become

$$
M_{c}^{0}(q)=\Theta\left(2 k_{F}-|q|\right), \quad N_{\downarrow}^{0}(p)=\Theta\left(k_{F \downarrow}-|p|\right)
$$

in the limit $L \rightarrow \infty$. The single occupancy of $q$ - and $p$-states suggests that (pseudo)particles associated with these quantum numbers behave as fermions. Carmelo and collaborators have used this fact to construct a formalism which allows an interpretation of the Bethe-Ansatz solution in terms of a generalized Fermi liquid of charge- and spinpseudo-particles, and proposed the name "Landau-Luttinger liquid" to integrable quantum systems exhibiting this structure [168], [181]-[184]. The low-energy physics is fully controlled by departures of the distribution functions from their ground state forms (4.92).

Consider a Hubbard model off half-filling in a magnetic field. The $S O(4)$-symmetry is therefore broken down to $U(1) \times U(1)$. In this case, all low-energy excitations of the Hubbard model are given by real roots $\left\{k_{i}\right\},\left\{\Lambda_{\alpha}\right\}$ of the Lieb-Wu equations (4.25) and (4.26), and are functionals of the distributions $M_{c}(q)$ and $N_{\downarrow}(p)$. Quantities like the energy $E$ or the momentum $P$ of a state, Eq. (4.28), or magnetization and particle number, are therefore functionals of $M_{c}(q)$ and $N_{\downarrow}(p)$, too. All low-energy states only have small deviations $\delta_{c}(q), \delta_{\downarrow}(p)$ from their ground state distributions

$$
M_{c}(q)=M_{c}^{0}(q)+\delta_{c}(q), \quad N_{\downarrow}(p)=N_{\downarrow}^{0}(p)+\delta_{\downarrow}(p) .
$$

The smallness of $\delta_{c}(q)$ and $\delta_{\downarrow}(p)$ allows an expansion of the energy in powers of these deviations $E=E_{0}+E_{1}+E_{2}+\ldots$ Here, $E_{0}$ is the ground state energy, and

$$
\begin{aligned}
E_{1}= & \frac{L}{2 \pi}\left\{\int_{-\pi}^{\pi} d q \delta_{c}(q) \varepsilon_{c}(q)+\int_{-k_{F \uparrow}}^{k_{F \uparrow}} d p \delta_{\downarrow}(p) \varepsilon_{\downarrow}(p)\right\}, \\
E_{2}= & \frac{L}{(2 \pi)^{2}}\left\{\int_{\pi}^{\pi} d q \int_{-\pi}^{\pi} d q^{\prime} \delta_{c}(q) \frac{f_{c c}\left(q, q^{\prime}\right)}{2} \delta_{c}\left(q^{\prime}\right)\right. \\
& +\int_{-k_{F \uparrow}}^{k_{F \uparrow}} d p \int_{-k_{F \uparrow}}^{k_{F \uparrow}} d p^{\prime} \delta_{\downarrow}(p) \frac{f_{s s}\left(p, p^{\prime}\right)}{2} \delta_{\downarrow}\left(p^{\prime}\right) \\
& \left.+\int_{-\pi}^{\pi} d q \int_{-k_{F \uparrow}}^{k_{F \uparrow}} d p \delta_{c}(q) f_{c s}(q, p) \delta_{\downarrow}(p)\right\}
\end{aligned}
$$

in precise analogy to the Fermi liquid [Eq. (2.1)]! The quantities $\varepsilon_{c}(q)$ and $\varepsilon_{s}(p)$ are the renormalized pseudo-particle energies, and the functions $f_{c c}, f_{s s}$, and $f_{c s}$ describe the pseudo-particle interactions. The momentum, particle number, and magnetization are 
linear in the deviations and therefore independent of the pseudo-particle interactions. Also the low-lying excitations involve only a single pseudo-particle, and the interaction term is of order $1 / L$ with respect to the kinetic energy and unimportant. On the other hand, the asymptotic decay of correlation functions is controlled by finite densities of pseudoparticles with low energy, and therefore determined by the pseudo-particle interactions $f$. These interactions can be related to both the elements of the dressed charge matrix $Z$ (4.43) and to the scattering phase shifts at the Fermi surface [122]. The influence of the interaction $U$ and the external fields $H$ and $\mu$ on the low-energy properties essentially is through the pseudo-particle interactions $f$.

Though extremely similar in structure to the Fermi liquid, these Landau-Luttinger liquids differ in some important ways. Unlike the Fermi liquid, the pseudo-particles describe collective charge and spin modes of the physical system (Section 4.4.2), and a construction of the physical electrons in terms of these pseudo-particles has not been achieved yet. Single-particle excitations constructed out of one holon and one spinon do not map onto free electrons as $U \rightarrow 0$. Finally, the pseudo-particle excitations here refer to exact eigenstates of the Hubbard Hamiltonian whereas the Fermi liquid quasi-particles are made from a superposition of such eigenstates and therefore decay with time.

Similarities and differences to the Fermi liquid can be gauged quite accurately from a study of two-particle excitations [168, 183]. The dynamical charge- or spin-susceptibility $(\nu=\rho, \sigma)$ has the spectral decomposition

$$
\chi^{(\nu)}(\mathbf{k}, \omega)=-\sum_{j}|\langle j|\nu(\mathbf{k})| 0\rangle|^{2} \frac{2 \omega_{j 0}}{\omega_{j 0}^{2}-(\omega+i 0)^{2}},
$$

where $|j\rangle$ is an eigenstate with energy $\omega_{j 0}$ relative to the ground state $|0\rangle$. Carmelo and Horsch observe that even in a Fermi liquid, in the limit $\mathbf{k} \rightarrow 0$, only matrix elements involving single-pair excitations connect to the ground state [168, 183]. In this limit, the pair excitations become real one-electron-one-hole excitations, and the corresponding matrix element with the ground state reduces to unity. This fact can be taken as a two-particle criterion for Fermi liquids. Unlike the quasi-particle residue, these matrix elements (there are four of them, taking $\rho$ and $\sigma$ between states with holon or spinon excitations and the ground state) do not vanish in the generalized Landau-Luttinger liquids, and are determined by the pseudo-particle interactions $f$. The matrix formed by these elements regularly tends towards the unit matrix as $U \rightarrow 0$, indicating that the long-wavelength two-particle properties of the Landau-Luttinger liquid smoothly evolve out of those of the free Fermi gas as the interactions are turned on. Adiabatic continuity therefore holds in the long-wavelength two-particle excitations. From a study of the charge and spin currents, one can determine both the charge and spin Drude weights of the conductivities, but also the charge and spin of the pseudo-particles themselves. All except the spinon charge (zero) depend on $U$ and the external fields and are not fixed to canonical values.

From the Fermi liquid character of the pseudo-particle excitations, we expect that we can find a framework similar to Chapter 3 for their low-energy description, and this is indeed the case [184]. In particular, one can formulate operator descriptions of these 
pseudo-particles, separately in the charge and spin sectors, which obey to a fermionic algebra. The low-energy structure can also be analyzed with conformal field theory, where one finds the typical tower structure of charge, current and sound excitations both for charges and spin. The particle-hole excitations are described by a $U(1)$-Kac-Moody algebra with central charge $c=1$, and the Virasoro generators can be constructed explicitly from the currents. Of course, one can then construct an effective boson description of the fermionic pseudo-particles.

However, one always has to remember that the pseudo-particles are not perturbatively related to physical particles, and their quantum numbers are not the quantum numbers of real excitations. While they clarify the structure of the theory to a considerable extent, they still do not allow for a straightforward computation of the physical correlation functions.

The single-particle properties of Luttinger liquids are distinctively different from Fermi liquids. Their two-particle properties are distinct at larger wavevectors, but in the centre of the Brillouin zone, they are very similar. In this sense, they can be considered as almost Fermi liquids. The notion of a Landau-Luttinger liquid is one formal way of making these connections explicit. 


\section{Chapter 5}

\section{Alternatives to the Luttinger liquid: spin gaps, the Mott transition, and phase separation}

The Luttinger liquid is one possible low-energy state of 1D fermions, realized when there is no gap in the excitation spectrum. There are several other possibilities: states with a gap in the spin excitations or/and in the charge excitations (the Mott insulator) or phase separation. (A more detailed review of the Mott transition in 1D has been written by Schulz [185].) In many models, there is a duality between the spin and charge degrees of freedom, and consequently the methods to describe them are closely related.

\section{$5.1 \quad$ Spin gaps}

Spin gaps occur in spin-rotationally invariant models when the electron-electron backscattering is effectively attractive. We have seen an example for renormalization group scaling in this situation in Section 4.3, Eqs. (4.11), when $g_{1}<0$. Scaling was towards strong coupling indicating that the Luttinger liquid fixed point was unstable but naturally, renormalization group alone cannot tell us much about the physics in this situation. The generic model for this problem is given by the Hamiltonian (3.1) plus (4.6) with $g_{1 \perp}<0$, and an easy solution was provided by Luther and Emery [186. For explicit spin-rotation invariance, we add a process $g_{1 \|}$ to the Hamiltonian, whose effect is to renormalize the $g_{2 \nu} \rightarrow g_{2 \nu}-g_{1 \|} / 2$. After the canonical transformation (3.28), $H_{\sigma}$, Eq. (3.31) is diagonal with a renormalized velocity $v_{\sigma}$ (3.33), and using (3.50), $H_{1 \perp}$ becomes

$$
H_{1 \perp}=\frac{2 g_{1 \perp}}{(2 \pi \alpha)^{2}} \int d x \cos \left[\sqrt{8 K_{\sigma}} \Phi_{\sigma}(x)\right] .
$$

The essential observation now is that for $K_{\sigma}=1 / 2$, i.e. $g_{1 \|}-2 g_{2 \sigma}=-6 \pi v_{F} / 5$, a sizable attractive interaction for a spin-rotation invariant model $\left(g_{2 \sigma}=0\right)$, (5.1) can be written as a bilinear in spinless fermions (3.57)

$$
H_{1 \perp}=\frac{g_{1 \perp}}{2 \pi \alpha} \int d x\left[\Psi_{+}^{\dagger}(x) \Psi_{-}(x) e^{2 i k_{F} x}+\text { H.c. }\right]
$$


in an external potential $\left(g_{1 \perp} / \pi \alpha\right) \cos \left(2 k_{F} x\right)$. The kinetic energy $\left(2 \pi v_{\sigma} / L\right) \sum_{r p} \sigma_{r}(p) \sigma_{r}(-p)$ can also be written in fermion representation, and the total Hamiltonian

$$
H^{\prime}=v_{\sigma} \sum_{r, k} r k c_{r, k}^{\dagger} c_{r, k}+\frac{g_{1 \perp}}{2 \pi \alpha} \sum_{k}\left(c_{+, k}^{\dagger} c_{-, k-2 k_{F}}+\text { H.c. }\right)
$$

can be diagonalized by a Bogoliubov transformation. In (5.3), $k \approx r k_{F}$. From the sineGordon form of the boson representation (5.1), it is apparent that the $\Psi_{r}^{\dagger}(x)$ create solitons rather than electrons. The eigenvalue spectrum is

$$
E_{r, \pm}(k)=v_{\sigma} k_{F} \pm \sqrt{\left(k-r k_{F}\right)^{2}+\Delta_{\sigma}^{2}} \quad, \quad \Delta_{\sigma}=\frac{g_{1 \perp}}{2 \pi \alpha},
$$

where $\Delta_{\sigma}$ is the spin gap at the Fermi level.

When $K_{\sigma} \neq 1 / 2$, the problem can no longer be solved exactly. Renormalization group arguments, however, support the existence of a spin gap

$$
\Delta_{\sigma} \sim \frac{v_{F}}{\alpha} \exp \left(\frac{\pi v_{F}}{g_{1}}\right)
$$

for all negative values of $g_{1 \|}=g_{1 \perp}$ and, with a different functional dependence, for all $g_{1 \perp}<0, \quad g_{1 \|}<\left|g_{1 \perp}\right|[76]$. This conclusion is reached by scaling the model onto the Luther-Emery line $K_{\sigma}\left(\ell_{L E}\right)=1 / 2$ and relating the gap to the Luther-Emery gap $\Delta_{L E}$ by the length of the scaling trajectory $\Delta_{\sigma}=\Delta_{L E} \exp \left(-\ell_{L E}\right)$. The gap may also be obtained from a homogeneity requirement of the partition function [187] or by using the exact solution of the sine-Gordon model [140].

The Hamiltonian (3.49) plus (5.1) is recognized as the quantum-sine-Gordon model which is equivalent to the massive Thirring model [40, 188]. Both models are related to the spin-1/2 Heisenberg chain [140, 141], and it is not surprising that they can be solved by Bethe Ansatz resp. the quantum-inverse-scattering method [27, 28, 87, 189, 190]. Haldane constructed a renormalized Bethe Ansatz solution and could determine some correlation functions of these models 191.

This gap has dramatic consequences for the physical properties. It implies long-range order in the $\Phi_{\sigma}$-field. This is best seen by going back to the boson representation of $H_{\perp}$ which has the form of a quantum-sine-Gordon Hamiltonian. For $g_{1 \perp}$ negative and scaling to strong coupling, the energy will be minimized by $\left\langle\cos \left(\sqrt{8} \Phi_{\sigma}\right\rangle=1\right.$, i.e. $\sqrt{8} \Phi_{\sigma}=$ $0 \bmod 2 \pi$. The $\Theta_{\sigma}$-field gets disordered, and correlation functions containing exponentials of $\Theta_{\sigma}$ will decay exponentially with a correlation length $\xi_{\sigma}=v_{\sigma} / \Delta_{\sigma}$. This cuts off the divergences in the SDW and TS correlation functions, while SS and CDW continue to diverge. Their exponents can be obtained by setting formally $K_{\sigma}=0$ in (3.92) and (3.99). (Due to the breaking of the $S U(2)$ spin-symmetry to $U(1)$ by our abelian bosonization scheme, the cutting off of the divergence is obvious only for the $S_{z}= \pm 1$-components of TS and the $x$ - and $y$-component of SDW. The representation of the $\mathrm{TS}_{0}$ and $\mathrm{SDW}_{z}(\overline{3.96})$ rather suggests a cancellation of two individually divergent terms with ordered $\Phi_{\sigma}$-fields, which is also found in a renormalization group calculation of the correlation functions [88. The conclusion of non-diverging TS and SDW correlations in all components is firm, however, and required by spin-rotation invariance.) 
The negative- $U$ Hubbard model falls into this universality class, and the spin gap can also be calculated from the Bethe Ansatz [103]. A physical mechanism for the generation of attractive interactions is electron-phonon interaction, and a renormalization group treatment of this problem has been presented in Section 4.5 [154. In an incommensurate system where repulsively interacting electrons are coupled to phonons of finite frequency, both the electron-phonon coupling and the phonon frequency determine if the system scales towards a Luttinger liquid fixed point or into a strong-coupling region with a spin gap. The first alternative has been discussed in Section 4.5. If the phonon frequency $\omega_{p h}$ is small and the coupling constant $\gamma_{1}$ big enough, the system will pass beyond the Luttinger liquid fixed point $g_{1 \perp}^{\star}=0, K_{\sigma}^{\star}=1$ before all retardation effects are scaled out, and flow into the spin gap region. The scaling out of the retardation effects then provides another factor $\omega_{p h} / E_{F}$ on the right-hand side of (5.5), and $g_{1} \rightarrow g_{1}\left(\ell=\ln \left[E_{F} / \omega_{p h}\right]\right)$ in the exponent in (5.5). Usually, CDW correlations are dominant, except for a Holstein-type electron-phonon coupling at sufficiently high phonon frequency, where superconductivity is found [157]. Here, the electron-phonon system is in the universality class of the LutherEmery model. If the phonon frequency is low enough, it may be more appropriate to start out from the Peierls mean-field limit [5], and correct it by quantum fluctuations and interactions 154. In any case, the formation of a 3D CDW is preceded by the opening of a 1D spin gap on the chains.

Examples for opening of spin gaps when the spin- $S U(2)$ is broken, are given by Giamarchi and Schulz [192.

\subsection{The Mott transition}

In half-filled bands, a Mott metal-insulator transition may occur as a results of commensurability, manifest in Umklapp scattering (4.8) becoming relevant. The problem is completely analogous to the spin-gap situation discussed above. In the special case of the Hubbard model at $n=1$, there is a duality transformation relating positive to negative $U$

$$
c_{i \uparrow} \rightarrow(-1)^{i} c_{i \uparrow}^{\dagger} \quad, \quad c_{i \downarrow} \rightarrow c_{i \downarrow} \quad
$$

i.e. a particle-hole transformation on one spin species only. In the boson representation, $U \rightarrow-U$ simply leads to an exchange of the roles of charge and spin fluctuations. All results of the preceding section then carry over, and the spin gap becomes the MottHubbard charge gap, separating the upper and lower Hubbard (sub)bands.

This picture can be extended to include the effects of doping (in the spin-gap problem, this corresponds to the introduction of a magnetic field which, however, is required to be unrealistically strong to have visible effects). We take the doping level $\delta=1-n$ and, due to charge conjugation symmetry at $n=1$, do not distinguish electron from hole doping. Due to the commensurability pinning, it is expected that $k_{F}$ does not respond immediately to doping, and that one will rather create charged defects in a commensurate SDW background. Therefore the gap structure is expected to persist for some finite doping range, but the chemical potential will move above (below) the gap to accommodate the 
additional charge carriers.

A more refined formulation of the model is necessary, however, to obtain a detailed picture of the Mott transition [44]. We consider the Hamiltonian of the charge degrees of (3.1) plus (4.8) and apply the canonical transformation (3.28). Unlike Section 3.2.1, we do not require the non-diagonal terms $\left(2 g_{2 \rho} / L\right) \sum_{p} \rho_{+}(p) \rho_{-}(p)$ to vanish but just transform so that $K_{\rho}=1 / 2$. In general then, a finite $g_{2}$-type interaction, not diagonal in the bosons, will remain. The Hamiltonian then becomes 141

$$
\begin{aligned}
H & =H_{0}+H_{1}, \\
H_{0} & =\sum_{k}\left[(v k+\mu): c_{+, k}^{\dagger} c_{+, k}:-(v k-\mu): c_{-, k}^{\dagger} c_{-, k}:\right]+\frac{g_{3 \perp}}{2 \pi \alpha} \int d x\left[c_{+, k}^{\dagger} c_{-, k}+\text { H.c. }\right], \\
H_{1} & =\frac{\pi v_{\rho} \sinh (2 \theta)}{L} \sum_{p}\left(2 \rho_{+}(p) \rho_{-}(-p)-f_{1} \sum_{r}: \rho_{r}(p) \rho_{r}(-p):\right), \\
v & =v_{\rho}\left(\cosh (2 \theta)+f_{1} \sinh (2 \theta)\right), \quad \exp (-2 \theta)=2 K_{\rho} .
\end{aligned}
$$

The fermions $c_{r k}$ are spinless, and the boson operators $\rho_{r}(p)$ refer to these spinless fermions. $H_{0}$ is of the Luther-Emery form and can be diagonalized. For the half-filled system, the interactions simply renormalize the gap as in the preceding section. The $f_{1}$ term has been introduced by Schulz 193 and does not affect the gap. $f_{1}$ is arbitrary and can be fixed as convenient.

At half-filling, the chemical potential is in the centre of the gap, and the lower (upper) band is completely filled (empty). Doping will shift it into the upper $(\delta<0)$ or lower $(\delta>0)$ subband generating a finite occupation of negative or positive carriers there. For very low energies $\left(\ll \Delta_{\rho}\right)$, the physics is determined by the partially occupied band only. The band structure can then be linearized again around the new Fermi level $k_{c}=\pi|\delta|$, and one keeps only interaction processes at the new Fermi surface. $f_{1}$ can now be fixed so as to cancel all $g_{4}$-type terms arising, and the new subband Hamiltonian is [44]

$$
\begin{aligned}
H & =\sum_{k} v_{c} k\left(a_{+, k}^{\dagger} a_{+, k}-a_{-, k}^{\dagger} a_{-, k}\right)+2 \pi v_{\rho} \sinh (2 \theta) f\left(k_{c}\right) \sum_{p} \rho_{+}^{(a)}(p) \rho_{-}^{(a)}(-p), \\
v_{c} & =\frac{\partial E}{\partial k}=\frac{v^{2} k_{c}}{\sqrt{\left(v k_{c}\right)^{2}+\left(\Delta_{\rho} / 2\right)^{2}}} \\
f_{1} & =\frac{1}{\sqrt{1+\left(2 v k_{c} / \Delta_{\rho}\right)^{2}}}, \quad f\left(k_{c}\right)=\frac{v_{c}^{2}}{v^{2}}
\end{aligned}
$$

The fermions $a_{r k}$ now refer to the partially occupied subband, and the $\rho_{r}^{(a)}(p)$ are constructed from these fermions. This spinless Hamiltonian can now be diagonalized as in Section 3.2.2.1, and the exponent $K$ governing the decay of correlation functions is given by

$$
K=\frac{1}{2}\left[1-\frac{4 v_{\rho} k_{c}}{\Delta_{\rho}} \sinh (2 \theta)\right] .
$$

Notice the following: (i) As one goes towards the half-filled band $\delta \rightarrow 0$, the Fermi velocity in the partially occupied band vanishes $\left(v_{c} \sim k_{c} / \Delta_{\rho} \rightarrow 0\right)$ which could be interpreted as 
a diverging effective mass $m \sim 1 / \delta$ 194 as $v_{c}=k_{c} / m$ in the vicinity of the Fermi surface. However, caution is required with this argument, because when only a few holes are left, the Fermi sea and velocity become ill-defined. The vanishing of $v_{c}$ indicates that one has reached the bottom of the upper subband, and there one recovers the parabolic dispersion of free particles with a finite effective mass. (ii) The interactions between the spinless fermions vanish $\propto \delta^{2}$ on account of the $f\left(k_{c}\right)$-factor, i.e. one is always in the weak-coupling limit close to the half-filled band. This ultimately justifies the separation of the Hamiltonian as in Eqs. (5.7). (iii) $K \rightarrow 1 / 2$ as $\delta \rightarrow 0$, which is consistent with the behaviour of the Hubbard model, Section 4.4. We do however not make use of any specific feature of this model, so that these results are valid for any Luttinger liquid close to half-filling [4]. (iv) Densely packed, strongly coupled spin-1/2 fermions map onto dilute, weakly coupled spinless fermions (holons, solitons). This kind of mapping can be fruitfully applied to many other problems [196. (v) Of particular interest is the Drude weight $D$ of the conductivity which, as was pointed out by Kohn [43], can be taken as an order parameter for the metal-insulator transition. The spinless fermion current is proportional to the one of the spin-carrying fermions, and the Drude weight is therefore $D \propto v_{c} K$, vanishing linearly as $\delta \rightarrow 0$ with a slope $\sim 1 / \Delta_{\rho}$. (vi) From the mapping onto the $2 \mathrm{D}$ commensurate-incommensurate transition, it follows that the Mott-Hubbard transition is in the universality class of the Pokrovsky-Talapov transition [193, 194, 195]. This is the case quite generally for the doping behaviour of models which, exactly at commensurability, display a Kosterlitz-Thouless transition as a function of the coupling constant, Eq. (4.11), and therefore applies universally to the metal-insulator transition at even commensurability ratios in $1 \mathrm{D}$.

At higher energies, the other (completely occupied) band contributes to the properties. To treat this case, Gulácsi and Bedell have proposed a bosonization scheme which decomposes the physical fermion into four new particles, a right- and left-moving fermion for each band 194. The Hamiltonian then takes the form of a Luttinger model for the partially occupied band and of a sine-Gordon model for the gapped band. One can then calculate various correlation functions and, concerning e.g. the momentum distribution function $n(k)$, finds a sum of a Luttinger function (3.86), weighted by the doping level $\delta$, and a term linear in $k$ characteristic for gapped systems [57, rather independent of doping.

A mapping of strongly coupled spinning fermions onto weakly interacting spinless holons can also be operated in the Bethe-Ansatz formalism for the Hubbard model [197]. The Bethe-Ansatz equations can be reformulated in terms of the charge excitations only and, for small doping, can be mapped onto weakly coupled holons. The Bethe Ansatz allows the introduction of a magnetic flux through the Hubbard ring, and the Drude weight can then be obtained from the second derivative of the ground state energy, Eq. (4.54). Also, the total optical spectral weight

$$
\pi N_{\text {tot }} \equiv \int_{0}^{\infty} \operatorname{Re}[\sigma(\omega)] d \omega=\frac{\pi}{2 L}\langle T\rangle=\frac{\pi}{2 L}\left(E_{0}-U \frac{\partial E_{0}}{\partial U}\right)
$$

can be computed quite easily, so that the optical properties can be discussed in some 
detail.

At half-filling, on a ring of circumference $L$, the Drude weight varies exponentially $D(L) \sim \exp [-L / \xi(U)]$ as $L \rightarrow \infty$, defining a coherence length $\xi(U) . \xi(U) \sim 1 / \Delta_{\rho}$ for $U \rightarrow 0$ but vanishes only logarithmically $\xi \sim 1 / \ln (U)$ for $U \rightarrow \infty$. $\xi$ also determines the exponential decay of the Green function at $n=1$, and comparing to the sine-Gordon form of the Hubbard Hamiltonian, is identified as the typical length of the solitons introduced upon doping. The divergence of $\xi$ as $U \rightarrow 0$ suggests that $U=0, n=1$ is a quantum critical point, and power-counting gives $D$ the scaling dimension zero. Consequently, the singular part of $D$ should be a dimensionless scaling function

$$
D^{\text {sing }}(n, L, U)=Y_{ \pm}(\xi \delta, \xi / L)
$$

where the index \pm refers to $U>(<) 0 . \quad Y_{ \pm}$can be determined both analytically and numerically in various limits. One remarkable result is that $D$ has a universal jump of $2 / \pi$ as $U$ goes from $0^{+}$to $0^{-}$at $n=1$ and $L=\infty$ - the system is insulating at positive $U$ and metallic at $U<0$. Moreover, at small doping, $Y_{+}(\xi \delta, 0) \sim \xi \delta$ as $\xi \delta \rightarrow 0$. The Drude weight grows linearly with doping, as we have already seen above. It saturates for $\xi \delta \sim 1$. On the other hand, the sum rule (5.10) is rather independent of $\delta, L$ and $U$ in the critical region. At $\delta=0$, all the spectral weight is in the upper Hubbard band. As one dopes the system, spectral weight is simply transferred from the upper to the lower Hubbard band where it goes into the Drude peak, a result which also holds in higher dimensions [198. When $\xi \delta \sim 1$, most of the spectral weight resides in the Drude peak, and the gap structure has been destroyed.

The charge velocity vanishes as $\delta \rightarrow 0$ (the Bethe Ansatz explicitly gives a finite effective mass to the holons). Close to the metal insulator transition, the charge entropy then is much higher than the spin entropy and will dominate the thermopower. This can then be evaluated from the spinless fermions [199], and one finds a hole-like thermopower for $n<1$ and an electron-like sign for $n>1$ with coefficients varying as $1 / \delta^{2}$ [42, 197]. On the other hand, the Fermi surface is given by the number of electrons in agreement with Luttinger's theorem [4].

Kolomeisky used renormalization group to provide a general framework of the Mott transition in a $1 \mathrm{D}$ metal of spinless fermions in an external potential with periodicity $k_{F} a=(p / q) \pi(p, q$ integers) [200, 201]. There is forward and backward single-particle scattering from this potential, and the electron-electron interaction is of spinless Luttinger form (hslf). Forward scattering, though, can be eliminated by the same argument as in (4.69) so long as one is interested only in the conductance which can be used as an order parameter for the transition. The backscattering terms are then mapped onto a model for the commensurate - incommensurate transition on 2D surfaces, which occurs at a critical $K_{c}=2 / q^{2}$ [202]. This mapping again identifies the universality classes of the Mott transition. Unlike the Hubbard model, a finite critical interaction strength $K_{c}<1$ is necessary for the transition to occur. We can approach it two different ways: (i) one can decrease $K \rightarrow K_{c}$ at fixed band-filling $k_{F} a=(p / q) \pi$ (corresponding to a soliton density $n_{s}=q k_{F} / \pi-p a=0$ in the 2D problem) (Kosterlitz-Thouless universality class [77]); 
(ii) one can vary the bandfilling $k_{F} a \rightarrow(p / q) \pi\left(n_{s} \rightarrow 0\right)$ at fixed $K<K_{c}$ (PokrovskyTalapov university class [195]). In both cases, there is a universal jump of $K$ and thus of the conductance at the transition. The insulating phase of course has $G \equiv 0$, and in the metallic phase

$$
\begin{aligned}
G & =\frac{e^{2}}{h}\left(K+\frac{\left|n_{s}\right|^{2\left(q^{2} K-2\right)}}{q^{2}}\right), \quad\left(K>K_{c}\right), \\
G & =\frac{e^{2}}{h}\left(\frac{2}{q^{2}}-\frac{\text { const. }}{q^{2} \ln \left|n_{s}\right|}\right), \quad\left(K=K_{c}\right), \\
G & \rightarrow \frac{e^{2}}{h} \frac{1}{q^{2}}, \quad\left(K<K_{c}, n_{s} \rightarrow 0\right) .
\end{aligned}
$$

Eq. (5.12) gives the conductance in case (i) above the transition, and here as well as in (5.13), we have indicated the corrections predicted for slightly doping $\left(n_{s} \ll k_{F} a\right)$ away from the ideal commensurability. Of course, $K \geq K_{c}$ includes the renormalization by the irrelevant scattering processes from the lattice (in total analogy to the logarithmic corrections found in Section 4.3). These are responsible for the nonanalytic correction terms.

The universal jumps of the conductance can also be rationalized quite easily in a language closer to the main development of this article. One would describe commensurability effects in the presence of electron-electron interaction by introducing suitable Umklapp operators $H_{3}^{(q)} \sim \int d x \cos [2 q \Phi(x)]$, transferring $q$ electrons across the Fermi surface, generalizing Section 4.3. Their scaling dimension will depend on $q$ as $2-q^{2} K$ and therefore imply a critical value of $K_{c}(q)=2 / q^{2}$ for each $q$. The universal jump of the conductance is then immediately obtained by inserting $K_{c}(q)$ into (4.77). Little work has been done on the problem in the presence of spin. At q-even commensurabilities, the above analysis is extended straightforwardly: the $2 q k_{F}$-transfer Umklapp operators become $H_{3}^{(S=0, q)} \sim \int d x \cos \left[\sqrt{2} q \Phi_{\rho}(x)\right]$, where in the superscript we have indicated the fact that the $q$ particles transfer a total spin $S=0$. These operators are more relevant than those transferring finite spin and have scaling dimensions $2-q^{2} K_{\rho} / 2$. The critical $K_{\rho}$ scales as $K_{\rho, c}=4 / q^{2}$. The half-filled Hubbard model and the half- and quarter-filled extended Hubbard models [50, 139, 142] obey to this relation. An example of an Umklapp operator transferring finite spin is given by

$$
H_{3 \|}=\frac{2 g_{3 \|}}{(2 \pi \alpha)^{2}} \int d x \cos \left[\sqrt{8} \Phi_{\rho}(x)\right] \cos \left[\sqrt{8} \Phi_{\sigma}(x)\right]
$$

which couples charge and spin. It is important in the half-filled extended Hubbard model where the CDW-SDW transition is continuous at weak coupling but becomes first order beyond a tricritical point at about $U=2 \mathrm{~V} \approx 4 \ldots 5 t$. At the origin is $H_{3 \|}$ with a scaling dimension $\left(2-2 K_{\rho}-2 K_{\sigma}\right)$ [50, 139]. For $q$-odd commensurabilities, half-odd-integer spin is necessarily transferred in Umklapp scattering. The Umklapp operators therefore must be of the form (5.15) although $\Phi_{\rho}$ and $\Phi_{\sigma}$ have prefactors different from $\sqrt{8}$. Here, charges and spins are strongly coupled, and the Mott transition is accompanied by the opening of a spin gap. The physics of such an odd- $q$ Mott insulator has not been explored yet. 
Transport in commensurate systems is very interesting because Umklapp processes provide an important relaxation mechanism for charge carriers. The problem here is that the conductivity does not have a regular perturbation expansion in the Umklapp operators $g_{3}$, Eq. (4.8). A way out is provided by the memory function formalism [203]. Assuming that the system is a normal metal with a finite dc-conductivity (a strong assumption which needs justification), one can rewrite Eq. (3.64) as

$$
\sigma(\omega)=\frac{2 i v_{\rho} K_{\rho}}{\pi} \frac{1}{\omega+M(\omega)}
$$

and a perturbative calculation of the memory function $M(\omega)$ is well-defined. It involves the commutator $[H, j]$ introduced before which, for the case of Umklapp processes in a half-filled band, Eq. (4.8), reads

$$
[j(x t), H]=\frac{8 g_{3 \perp}}{(2 \pi \alpha)^{2}} i v_{\rho} K_{\rho} \sin \left[\sqrt{8 K_{\rho}} \Phi_{\rho}(x t)+\delta x\right],
$$

where $\delta=4 k_{F}-2 \pi / a$ measures an eventual doping level with respect to the half-filled band.

If $g_{3 \perp} \ll 1$, one obtains $\sigma(\omega) \sim 1 / \omega$ for $K_{\rho}>1$ and $\sigma(\omega) \sim \omega^{3-4 K_{\rho}}$ for $K_{\rho}<1$ if $\omega \gg T$. In the opposite case $T \gg \omega$, one has $\sigma(0, T) \sim T^{3-4 K_{\rho}}$. Of course, these results are valid only at sufficiently high $T$ and $\omega$ because at smaller scales, there will be a charge gap and one expects an activated conductivity. In case that $g_{3 \perp}$ is finite, one can extend these results by performing a renormalization group calculation such as the one in Section 4.3 which will be stopped by the finite temperature at a scale $\ell_{T}=\ln \left(E_{F} / T\right)$. Then, additional temperature dependence in the conductivity will be generated by inserting the renormalized values of $g_{3 \perp}\left(\ell_{T}\right)$ and $K_{\rho}\left(\ell_{T}\right)$ into the memory functions.

Surprising results obtain at lower frequencies where one expects the influence of the charge gap. Here, one can use the Luther and Emery solution [186] to diagonalize the charge part of the Hamiltonian in terms of new spinless fermions, and then express the current in the new fermions [44. The dc-conductivity comes out infinite at any finite temperature. The explanation is quite obvious: the only scattering mechanism for our charge carriers were the Umklapp processes which, however, have been diagonalized exactly by the Luther-Emery transformation. Thermally excited carriers have no dissipation mechanism left.

Away from half-filling, there are two regimes. If $T, \omega \gg v_{\rho} \delta$, the energy scales are too high for the Umklapp processes to be quenched by doping, and one basically recovers the half-filled band results. If $T$ falls below $v_{\rho} \delta$, the latter quantity will act as a cutoff to renormalization and freeze out the Umklapp scattering. One will then have a crossover to presumably exponential increase with $1 / T$ of the conductivity characteristic for the incommensurate system. On the other hand, at zero temperature, as one approaches the half-filled band, the weight of the Drude $\delta(\omega)$-part vanishes linearly with doping and with a slope that depends on the charge gap [44, for the Hubbard model in agreement with the Bethe Ansatz results [197].

Similar results for $\sigma(\omega)$ can be obtained by using Eq. (3.68). On the other hand, the predictions for $\sigma(T)$ agree with the memory function approach 204 only in certain 
cases [45. Giamarchi and Millis suggest that the use of memory functions is particularly dangerous in cases of infinite dc-conductivity where the underlying conservation laws may not be incorporated correctly into this method 445. In fact, it seems that there is an obvious contradiction between the assumed finite dc-conductivity of the memory function method and the infinite conductivity in the Luttinger liquid.

The $q \approx 0$-component of the two-particle charge-charge spectral function $\operatorname{Im} R_{\rho}(q, \omega)$ with $R_{\rho}$ from Eq. (3.91)] can be studied in detail close to the Mott transition because the charge operator here has a simple representation in terms of the spinless fermions $\rho(x t)=\Psi_{+}^{\dagger}(x t) \Psi_{+}(x t)+\Psi_{-}^{\dagger}(x t) \Psi_{-}(x t)$ [205]. At finite doping $\delta$, one finds a two-peak structure: a low-energy peak with linear dispersion arises from the long-wavelength density fluctuations within the partially occupied subband. It looses weight $\propto \delta \rightarrow 0$ as the Mott transition is approached. This peak is modelled accurately by the effective Luttinger liquid description of Chapter 4 . However, a second peak at higher energies $\left(\sim \Delta_{\rho}\right)$ is quite pronounced over a significant doping range. It represents density fluctuations between the upper and lower Hubbard subbands. In the Mott insulating phase $(\delta=0)$, it is the only signal present. The region in $q$ and $\omega$ in which a simple Luttinger liquid description is valid, therefore is very small close to the Mott transition and may contain excitations carrying very little spectral weight 205].

Shankar has considered the effect of impurities in commensurate models [47. For a half-filled spinless fermion system with nearest-neighbour repulsion $V \sum_{i} n_{i} n_{i+1}$, which has a Mott transition into a CDW state at $V=2 t$, he finds that the Mott gap is destroyed by even a small amount of disorder. This is supported by numerical density matrix renormalization group [206]. On the other hand, this work points towards persisting differences between impurities in systems with and without a Mott gap: if one dopes the system with an additional charge, it is strongly localized when the interactions are strong enough to open a charge gap while localization is quite weak in the absence of the gap, for identical disorder configurations. This situation corresponds to a Mott transition as a function of band-filling at fixed interaction strength, and Kolomeisky has argued that the elementary excitations remain solitons, as in the pure Mott system, which, however, become localized in the presence of arbitrarily small disorder [207. In contrast, for a half-filled $(\mathrm{S}=1 / 2)$ Hubbard model, Shankar predicts the Mott-Hubbard charge gap $\Delta_{\rho}$ to survive so long as the variations of the random potential are bounded to $|\xi(x)| \ll \Delta_{\rho}$ in agreement with general arguments [207] and real-space renormalization group [208]. The difference may again be due to the impurities coupling linearly to the CDW fluctuations which build up the order parameter in the spinless model but which are suppressed in the half-filled Hubbard model.

In a different line of work, Horsch and Stephan [209] consider the conductivity of a single hole doped into a half-filled $t-J$ - and large- $U$ Hubbard model. They find that, in addition to the Drude peak, there is conductivity at finite frequencies varying as $\omega^{-1 / 2}$ and $\omega^{3 / 2}$, respectively. These analytical results are supported by numerical diagonalization studies on rings as big as 19 sites. Currently, it is not understood why $\sigma(\omega>0)$ in both models differs qualitatively and also differs from the Luttinger liquid prediction $\omega^{3}$. On the other hand, one could speculate that a crossover to the $\omega^{3}$ behaviour could occur as a 
finite concentration of holes is doped into the Mott insulator and a Fermi surface forms.

The Ogata-Shiba wavefunction Eq. (14.34) [107] also allows to find the spectral properties of one hole doped into a half-filled $U=\infty$-Hubbard model, the prototypical MottHubbard insulator. This problem had been examined long time ago by Brinkman and Rice [210] who assumed Néel order for the spin configuration. They had found a complete localization of the hole becoming totally incoherent. Expressed in terms of the spectral function

$$
A(k, \omega)=-\frac{1}{\pi} \operatorname{Im} G(k, \omega)=\frac{1}{2 \sqrt{\omega^{2}-4}} .
$$

There is no quasi-particle pole, and $A$ is independent of $k$. This result, however, is mainly due to the assumed static antiferromagnetic Néel order. In the $U \rightarrow \infty$-Hubbard model, the magnetic ground state is far from Néel, and taking the real Heisenberg ground state, Sorella and Parola get quite different spectral functions [120]. Generically, a three-peak structure is found which can be understood qualitatively as a convolution of a holon and spinon Green function $\left[\sim 1 /\left\{\omega-\varepsilon^{(h)}(k)\right\}\right.$ resp. $\sim 1 / \sqrt{\omega-\varepsilon^{(s)}(k)}$ 122], where $\varepsilon^{(h, s)}(k)$ has been defined in Eqs. (4.29) and (4.30)]. At special wavevectors, two singularities may coalesce giving the peaks observed. Unfortunately, qualitatively different results were published slightly later by the same authors [121] where only a two-peak behaviour is found. While the latter is in agreement with the spectral function of a Luttinger model with charge-spin separation only and no anomalous dimension $(\alpha=0$ - there is no partner for the holes to $g_{2}$-interact) [59, 61], the reason for the discrepancy between both results is not clear. The dynamics of a single hole in the 1D $t-J$-model has also been studied by Horsch and coworkers [211] both by numerical diagonalization, and analytically within the subspace generated by applying the hopping operator to the state obtained by annihilating a fermion with $(k, s)$ in the 1D Néel state. The two methods agree in their essential features. The inclusion of quantum fluctuations in the spin background changes the results in several essential ways with respect to the dispersionless, incoherent Brinkman-Rice continuum. (i) It generates interesting dispersion in the spectra. While the lowest eigenstate disperses on a scale $J$, the first moment of the spectral function $\int_{-\infty}^{\infty} d \omega \omega A(k, \omega)$ disperses on a scale $t$. (ii) To the extent one can gauge from the finite lattice data, there seem to be three peaks, and the above dispersion behaviour suggests that spectral weight is mainly transferred, as a function of $k$ between peaks which have different dispersion. This is not unlike the three-peak structure initially found by Parola and Sorella for the Hubbard model 120] but different from later work by the same authors [121]. (iii) The lower edge of the spectrum disperses little and remains sharp close to $\omega \approx-2 t$, but the high-energy edge of the Brinkman-Rice continuum gets washed out into a tail of states. Comparing the work of the different groups it appears that the dynamics of a single hole doped into a Mott insulator is not fully clarified.

\subsection{Phase separation}

For strongly attractive interactions $g_{2 \rho}+g_{4 \rho}=-\pi v_{F}, K_{\rho}$ becomes infinite, indicating an instability of the Luttinger liquid [9]. The physical interpretation of this transition 
becomes obvious by going back to Eqs. (3.32) and (3.62) which shows that a divergence in $K_{\rho}$ implies

$$
\kappa=\frac{\partial n}{\partial \mu} \rightarrow \infty \quad, \quad v_{N \rho} \rightarrow 0
$$

Both facts indicate that a particle can be added to the system without cost in energy; the electron clump into droplets i.e. one has phase separation. This transition takes place e.g. in the extended Hubbard model with nearest neighbour attraction [50, 139, 142, 143, 145] or in the $t-J$-model with sufficiently large $J$ [130, 131, 133]. 


\section{Chapter 6}

\section{Extensions of the Luttinger Liquid}

This chapter discusses three extensions of the Luttinger liquid picture. The first two extensions are intimately related: models with two or more bands, and models with several coupled chains. In the third part, we outline the important extension to chiral Luttinger liquids arising in the fractional quantum Hall effect, where a Luttinger liquid with central charge $c \neq 1$ is found.

There are very strong similarities between models with several bands and models with several chains. The former in fact often model the bandstructure of materials with several chains per unit cell. Coupling $\mathrm{N}$ chains by a hopping matrix element produces $\mathrm{N}$ bands. They also can originate from applying a strong magnetic field to a chain. We somehow artificially separate this topic into two parts on multi-component and multi-chain models mainly because the physical questions asked in both parts are rather different.

To see the similarities more closely, consider first a two-band model

$$
H=\sum_{k, s, \alpha} \epsilon_{\alpha}(k) c_{k s \alpha}^{\dagger} c_{k s \alpha}+H_{i n t}
$$

$\alpha$ is the band index, and $\epsilon_{\alpha}(k)$ is the dispersion. The Fermi momenta $k_{F \alpha}$ and velocities $v_{F \alpha}$ may be different in general, and the $v_{F \alpha}$ may be positive (electron) or negative (hole bands at the centre of the Brillouin zone) as shown in Fig. 6.1. $H_{\text {int }}$ is the interaction Hamiltonian which contains all the processes $g_{i}$ discussed before, both within every band $\alpha$ and between the different bands. For electrons in a magnetic field $H \| \hat{z}$, the single-particle Hamiltonian is

$$
H_{0}=\sum_{k s} \epsilon(k) c_{k s}^{\dagger} c_{k s}+h \sum_{k}\left(c_{k \uparrow}^{\dagger} c_{k \uparrow}-c_{k \downarrow}^{\dagger} c_{k \downarrow}\right) \quad, \quad h=g \mu_{B} H / 2 .
$$

With $s \rightarrow \alpha$, this reduces to a spinless variant of (6.1) with $\epsilon_{\alpha}(k)=\epsilon(k) \pm h$. Coupling two chains $i=1,2$ with a single-particle tunneling matrix element $t_{\perp}$ gives

$$
H_{0}=\sum_{k s i} \epsilon(k) c_{k s i}^{\dagger} c_{k s i}-t_{\perp} \sum_{k s}\left(c_{k s 1}^{\dagger} c_{k s 2}+\text { H.c. }\right) \text {. }
$$

Here the bonding and antibonding bands disperse with $\epsilon_{0, \pi}(k)=\epsilon(k) \mp 2 t_{\perp}$ and are labeled by their transverse momenta 0 and $\pi$. The spinless version of (6.3) also describes 
electrons in a transverse magnetic field $H \| \hat{x}$, and the dispersions $\epsilon_{0, \pi}(k)$ can then also be obtained by rotating the field around the $y$-axis align it with $\hat{z}$. Under this rotation, the interactions transform nontrivially. The transformation does not affect the "isocharge". In the "isospin" channel, the $g_{1}$-processes (and consequently also $g_{2 \sigma}$ ) transform as [213]

$$
\begin{aligned}
H_{1}= & -g_{1 \|} \int d x: \sigma_{+}(x) \sigma_{-}(x):+g_{1 \perp} \sum_{s} \int d x \Psi_{+, s}^{\dagger}(x) \Psi_{-,-s}^{\dagger}(x) \Psi_{+,-s}(x) \Psi_{-, s}(x) \\
\rightarrow & -g_{1 \perp} \int d x: \sigma_{+}(x) \sigma_{-}(x):+\frac{g_{1 \|}+g_{1 \perp}}{2} \int d x \Psi_{+, s}^{\dagger}(x) \Psi_{-,-s}^{\dagger}(x) \Psi_{+,-s}(x) \Psi_{-, s}(x) \\
& -\frac{g_{1 \|}-g_{1 \perp}}{2} \int d x \Psi_{+, s}^{\dagger}(x) \Psi_{-, s}^{\dagger}(x) \Psi_{-,-s}(x) \Psi_{+,-s}(x) .
\end{aligned}
$$

The last process does not conserve the total spin of the scattering partners. In a singleband model, it can arise from spin-orbit scattering, and work on this topic is relevant here [192]. The coupling constant is commonly denoted by $g_{f}$ and describes interband backscattering and does not conserve the number of particles on a given branch of a band. More interactions of this kind can arise in systems where the starting model has internal degrees of freedom. Of course, in all these multicomponent problems, the standard fluctuation operators from Section (3.3) can be extended to include inter-component fluctuations, giving a flavour of the richness of the physics that can be described.

\subsection{Multi-component models}

We first describe a multi-component Tomonaga-Luttinger model, and then some of the instabilities occurring in more complicated models when scaling does not go towards a Tomonaga-Luttinger fixed point in all channels.

The Hamiltonian for Tomonaga-Luttinger model with $N$ components (colours, labelled by $\lambda$, including spin and chirality index) is [126]

$$
\begin{aligned}
H & =\sum_{\lambda=1}^{N} H_{\lambda}+\sum_{\lambda, \lambda^{\prime}=1}^{N} H_{\lambda \lambda^{\prime}}, \\
H_{\lambda} & =v_{\lambda} \sum_{k}\left(k-k_{F \lambda}\right) c_{k \lambda}^{\dagger} c_{k \lambda}, \\
H_{\lambda \lambda^{\prime}} & =\frac{1}{2 L} \sum_{p} g_{\lambda \lambda^{\prime}}(p) \rho_{\lambda}(p) \rho_{\lambda^{\prime}}(-p) .
\end{aligned}
$$

We assume that the Fermi velocities and momenta are pairwise $\left(v_{\lambda},-v_{\lambda}\right),\left(k_{F \lambda},-k_{F \lambda}\right)$, corresponding to a symmetric dispersion, and that the coupling constants satisfy $g_{\lambda \lambda^{\prime}}=$ $g_{\lambda^{\prime} \lambda}$. The standard Tomonaga-Luttinger model (3.1) is obtained for $N=4$ and the two pairs of Fermi velocities and momenta equal. The density operators commute as

$$
\left[\rho_{\lambda}(p), \rho_{\lambda^{\prime}}(-p)\right]=-\frac{p L}{2 \pi} \delta_{\lambda, \lambda^{\prime}} \delta_{p, p^{\prime}} \operatorname{sign} v_{\lambda}
$$

One can now repeat the arguments of Section 3.2.1 to show that (i) the single-particle Hamiltonian $H_{\lambda}$ can be written as a boson bilinear, and (ii) that the equivalence to the 
boson description is complete only upon including charge $\left(N_{\lambda}\right)$ excitations with respect to the vacuum. The Hamiltonian then becomes

$$
\begin{aligned}
H & =\frac{2 \pi}{L} \sum_{\lambda, \lambda^{\prime}, p>0} A_{\lambda \lambda^{\prime}}(p) \rho_{\lambda}(p) \rho_{\lambda^{\prime}}(-p)+\frac{\pi}{L} \sum_{\lambda \lambda^{\prime}} A_{\lambda \lambda^{\prime}}(p=0) N_{\lambda} N_{\lambda^{\prime}}, \\
A_{\lambda \lambda^{\prime}}(p) & =\left|v_{\lambda}\right| \delta_{\lambda \lambda^{\prime}}+\frac{g_{\lambda \lambda^{\prime}}(p)}{2 \pi} .
\end{aligned}
$$

This Hamiltonian being a bilinear form in the bosons, it can be diagonalized by an $N$ component generalization of a Bogoliubov transformation 126

$$
\begin{aligned}
H= & \frac{2 \pi}{L} \sum_{j, p>0}\left|v_{j}\right| \rho_{j}\left(p \operatorname{sign} v_{j}\right) \rho_{j}\left(-p \operatorname{sign} v_{j}\right)+\frac{\pi}{L} \sum_{j}\left|v_{j}\right| \sum_{\lambda \lambda^{\prime}} N_{\lambda} \alpha_{\lambda \lambda^{\prime}}^{(j)} N_{\lambda^{\prime}}, \\
P= & \sum_{\lambda} k_{F \lambda} N_{\lambda}+\frac{\pi}{L} \sum_{j \lambda \lambda^{\prime}} \operatorname{sign}\left(v_{j}\right) N_{\lambda} \alpha_{\lambda \lambda^{\prime}}^{(j)} N_{\lambda^{\prime}}+ \\
& +\frac{2 \pi}{L} \sum_{j, p>0} \operatorname{sign}\left(v_{j}\right) \rho_{j}\left(p \operatorname{sign} v_{j}\right) \rho_{j}\left(-p \operatorname{sign} v_{j}\right) .
\end{aligned}
$$

$P$ is the momentum operator. The index $j$ denotes the new operators and parameters. The renormalized sound velocities $v_{j}$ and the matrix $\alpha_{\lambda \lambda^{\prime}}^{(j)}$ are obtained as the solution of the eigenvalue problem

$$
\begin{aligned}
\mathcal{A} \cdot \mathcal{B}\left|w^{(j)}\right\rangle & =v_{j}\left|w^{(j)}\right\rangle, \\
\mathcal{A} & =\left(A_{\lambda \lambda^{\prime}}\right), \quad \mathcal{B}=\left(\delta_{\lambda \lambda^{\prime}} \operatorname{sign} v_{\lambda}\right), \quad \alpha_{\lambda \lambda^{\prime}}^{(j)}=w_{\lambda}^{(j)} w_{\lambda^{\prime}}^{(j)} .
\end{aligned}
$$

Correlation functions

$$
\begin{gathered}
G_{\lambda_{1} \ldots \lambda_{m}}\left(\mathbf{x}_{1}^{\prime}, \ldots, \mathbf{x}_{m}^{\prime} ; \mathbf{x}_{1}, \ldots, \mathbf{x}_{m}\right)= \\
(-i)^{m}\left\langle T \Psi_{\lambda_{1}}\left(\mathbf{x}_{1}^{\prime}\right) \ldots \Psi_{\lambda_{m}}\left(\mathbf{x}_{m}^{\prime}\right) \Psi_{\lambda_{m}}^{\dagger}\left(\mathbf{x}_{m}\right) \ldots \Psi_{\lambda_{1}}^{\dagger}\left(\mathbf{x}_{1}\right)\right\rangle
\end{gathered}
$$

can then be evaluated either by generalization of the bosonization formula (3.41) to $N$ components, or by combining the Ward identities associated with (6.5) with equationof-motion methods. [In (6.12), $\mathbf{x}$ stands for the space-time point $(x, t)$ and $T$ is the time-ordering operator.] One finds

$$
\begin{aligned}
G_{\lambda_{1} \ldots \lambda_{m}}\left(\mathbf{x}_{1}^{\prime}, \ldots, \mathbf{x}_{m}^{\prime} ; \mathbf{x}_{1}, \ldots, \mathbf{x}_{m}\right)=\prod_{l} G_{\lambda_{l}}\left(\mathbf{x}_{l}^{\prime}, \mathbf{x}_{l}\right) \prod_{l^{\prime}<l} \frac{f_{\lambda_{l} \lambda_{l^{\prime}}}\left(\mathbf{x}_{l}-\mathbf{x}_{l^{\prime}}^{\prime}\right) f_{\lambda_{l} \lambda_{l^{\prime}}}\left(\mathbf{x}_{l}^{\prime}-\mathbf{x}_{l^{\prime}}\right)}{f_{\lambda_{l} \lambda_{l^{\prime}}}\left(\mathbf{x}_{l}-\mathbf{x}_{l^{\prime}}\right) f_{\lambda_{l} \lambda_{l^{\prime}}}\left(\mathbf{x}^{\prime}{ }_{l}-\mathbf{x}_{l^{\prime}}^{\prime}\right)} \\
G_{\lambda}\left(\mathbf{x}^{\prime}, \mathbf{x}\right)=G_{\lambda}^{(0)}\left(\mathbf{x}^{\prime}, \mathbf{x}\right) f_{\lambda \lambda}\left(\mathbf{x}-\mathbf{x}^{\prime}\right), \\
f_{\lambda \lambda^{\prime}}(x, t)=\Lambda^{-2 \alpha^{\prime}}\left[x-v_{\lambda} t+\frac{i}{\Lambda} \operatorname{sign}\left(v_{\lambda} t\right)\right]^{\delta_{\lambda \lambda^{\prime}}} \prod_{j}\left[x-v_{j} t+\frac{i}{\Lambda} \operatorname{sign}\left(v_{j} t\right)\right]^{-\alpha_{\lambda \lambda^{\prime}}^{(j)}}
\end{aligned}
$$

Here $G^{(0)}(\mathbf{x})$ is the noninteracting Green function, $\Lambda$ is the momentum transfer cutoff familiar from Section 3.1.2, and $\alpha^{\prime}=\sum_{j} \alpha_{\lambda \lambda^{\prime}}^{(j)}$ with the sum going only over those $j$ where $v_{j} v_{\lambda}<0$. Asymptotically, this gives

$$
G\left(\{\mathbf{x}\},\left\{\Delta N_{\lambda}\right\}\right) \sim e^{-i x \sum_{\lambda} \Delta N_{\lambda} k_{F \lambda}} \prod_{j}\left(x-v_{j} t\right)^{-2 \Delta_{j}}
$$


with

$$
\Delta_{j}=\sum_{\lambda \lambda^{\prime}} N_{\lambda} \alpha_{\lambda \lambda^{\prime}}^{(j)} N_{\lambda^{\prime}}
$$

$\Delta N_{\lambda}$ is the number of fermions of colour $\lambda$ propagating from $\mathbf{0}$ to $\mathbf{x}$, weighted by $\operatorname{sign} v_{\lambda}$, i.e. the charge excitation introduced by the operator whose correlations are to be computed. This is in agreement with the conformal field theory prediction, if the $\Delta_{j}$ are interpreted as scaling dimensions. That this is indeed justified is seen by evaluating energies and momenta in a state with a definite number of charge and particle-hole excitations $\left(N_{\lambda}, n_{j}=L q / 2 \pi\right.$ respectively)

$$
\begin{aligned}
E\left(N_{\lambda}, n_{j}\right)-E_{0} & =\frac{2 \pi}{L} \sum_{j}\left|v_{j}\right|\left(\Delta_{j}+n_{j}\right)+\ldots, \\
P\left(N_{\lambda}, n_{j}\right) & =\sum_{\lambda} N_{\lambda} k_{F \lambda}+\frac{2 \pi}{L} \sum_{j}\left(\Delta_{j}+n_{j}\right) \operatorname{sign} v_{j},
\end{aligned}
$$

i.e. one obtains the typical tower structure of conformal field theories. Comparing with Eqs. (3.161), (3.162), $\Delta_{j}$ is identified as the scaling dimension of a primary operator, and we thus have generalized these expressions to an $N$-component system [126].

One example where these expressions can be fruitfully applied, is the Hubbard model in a magnetic field which scales towards an $N=4$-Tomonaga-Luttinger model. Of course, one could take the perturbative renormalization approach [214]. More accurate results, valid at any coupling, are obtained, however, from the the Bethe-Ansatz solution in a finite magnetic field. There are two practical possibilities: either use conformal field theory directly to obtain the correlation exponents [127] or perform a mapping on the $N$-color Tomonaga-Luttinger model by identifying the low-energy spectral properties between both models [126]. We briefly comment on the second method.

The similarity to the $\boldsymbol{\Delta} \mathbf{N}$ and $\mathbf{D}$ in the conformal field theory treatment of the Hubbard model in Section 4.4 .3 should be apparent, as well as the similarity between Eqs. (6.14) and (4.45). The requirement that the correlation exponents of the TomonagaLuttinger model and the Hubbard model be equal, implies for the scaling dimensions

$$
\Delta_{j}=\Delta_{c(s)}^{ \pm}
$$

where the latter quantity is evaluated in the Hubbard model and related by (4.49) to the elements of the dressed charge matrix. Also the $\mathcal{A}$-matrix, and therefore the coupling constants $g_{\lambda \lambda^{\prime}}$ of the Tomonaga-Luttinger model, can be found. This basically involves constructing an expansion of the scaling dimensions (6.15) in terms of the excitations in the Bethe Ansatz wavefunction of the Hubbard model. Of course, the coupling constants $g_{i}$ are found linear in $U$ at small $U$. As $U \rightarrow \infty$, they saturate, as implied by the saturation in $K_{\rho}$. More interesting is the finding that, at fixed $U$, the $g_{i}$ have a nonanalytic variation with $h$ as $h \rightarrow 0$, translating into a nonanalytic $h$-dependence of $K_{\rho}$ [127], and meaning that a magnetic field $h$ can never be regarded as a small perturbation.

Models involving interactions other than forward scattering alone often do not scale towards the $N$-colour Luttinger liquid fixed point (6.5) and most often have been studied 
for two bands $(A, B)$ with or without spin degrees of freedom. The most important interaction not contained in single-band models is interband backscattering, the last term in (6.4) with coupling constant $g_{f}$. In Eq. (6.4), the spin index labels the two bands $s=A, B$. (There are more interband backscattering terms; momentum conservation suppresses all of them but $g_{f}$ when the two Fermi momenta are not nearly equal.) For spinning fermions, we can have $g_{f \|} \neq g_{f \perp}$. For half-filled bands (here $k_{F, A}+k_{F, B}$ or $\left.2 k_{F, A(B)}=2 \pi / a\right)$, one must further add the corresponding Umklapp scattering process $g_{3}$. It is also useful to think in terms of charge fluctuation interactions (scattering processes changing the number of particles in a band like $g_{f}$ does) and exchange interactions (interband processes conserving the charge but changing the spin in a band).

These models have been studied most often with the same methods used for the singleband problems: renormalization group starting from a bosonic or fermionic description and eventually strong-coupling field theory. We neglect from our subsequent discussion all non-Luttinger intraband interactions whose effects have been discussed in the preceding chapters. The 2-colour Luttinger liquid (6.5) is a hyperplane of critical fixed points $g_{3}=g_{f}=0$ [215]. It is stable for $2 g_{2 A B}<-\left|g_{2 A A}+g_{2 B B}\right|$, and then is attractive for $\left[g_{f}=0\right.$ and $\left.\left(2 g_{2 A B}+g_{2 A A}+g_{2 B B}\right)<c\left|g_{3}\right|\right]$ or $\left[g_{3}=0\right.$ and $\left.\left(2 g_{2 A B}-g_{2 A A}-g_{2 B B}\right)<-c\left|g_{f}\right|\right]$, where $c$ is a constant related to the difference in the Fermi velocities. In the symmetric case $\left(v_{F, A}=\right.$ $\left.v_{F, B}, g_{2 A A}=g_{2 B B}\right)$ the two bands decouple, and one can compute correlation functions by the standard methods presented earlier [192]. In the absence of intraband backscattering, the renormalization group equations have the Kosterlitz-Thouless structure (4.11), and there are both massive and massless phases. If $g_{2 A B}-g_{2 A A}<0$ and $\left|g_{2 A B}-g_{2 A A}\right|<\left|g_{f}\right|$, scaling will go to weak coupling, and there will be a massless two-component Luttinger liquid with dominant CDW correlations. The combination $g_{2 A B}+g_{2 A A}$ controls if they are of inter- or intraband type (corresponding to $\mathrm{SDW}_{z}$ and CDW of a $\mathrm{s}=1 / 2$-singleband model, respectively). If one of the preceding inequalities is violated, scaling will go to strong coupling and, depending on $g_{2 A B}+g_{2 A A}$, one will have either intraband superconducting pairing, or one of two new types of interband CDWs (corresponding to $\mathrm{SDW}_{x, y}$ in a spin-1/2 single-band model). Their structure can be seen more clearly if one imagines the two bands arising from two spinless fermion chains coupled by $t_{\perp}$ [213]. There, they correspond to (i) a CDW with a charge density modulation on the bonds of the chains and (ii) to a configuration where currents circulate around the plaquettes of the ladder in an alternating pattern. This can be viewed as an orbital antiferromagnet and is directly related to the staggered flux phases discussed some time ago in the high- $T_{c}$ -problem 216.

In the asymmetric model, the bands do not decouple. At weak coupling, this is quite apparent from the renormalization group equations. At strong coupling, the Hamiltonian can be decoupled into two sine-Gordon models involving phase fields which are linear combinations of those describing the bands, with one condition on the coupling constants [215], and the excitation spectra and correlation exponents can be determined. One consequence of the coupling between the bands is that the correlation exponents for the different intraband and the interband fluctuations may all be different. For example, when $g_{3}=0, g_{f}$ may become relevant and open a gap in one of the sine-Gordon models, 
the other remaining massless. Depending on the precise value of the interactions, either an intraband SS or an interband CDW have divergent fluctuations. It is interesting then that the conditions for divergent SS can be realized from purely repulsive interactions, something impossible in the single-band case [215].

Up to now, we have discussed only the physics of the charge fluctuations. The interband exchange processes are interesting, too, and require an extension of the previous models by spin degrees of freedom [212, 217]. Due to the proliferation of the coupling constants, a general discussion of such a model is a formidable task, and will not be attempted here. An interesting limit is $v_{A} \ll v_{B}$, i.e. a band of light electrons (B) coupled to heavy electrons (A). On a technical level, a small velocity $v_{A}$ in (6.1) is generated by hybridizing a dispersionless A-band with the B-electrons [take the two-chain Hamiltonian (6.3) and put $t_{\|}=0$ for one spin direction only]. For $v_{A} \ll v_{B}$, the charge fluctuations between the bands may scale out of the problem. In that case, exchange between the bands is the only remaining coupling, and the physics then becomes very similar to the single-impurity Kondo problem [212, 217]. In particular, for positive exchange coupling constants, scaling goes to weak coupling, in analogy to the ferromagnetic Kondo impurity. For negative exchange constants, on the other hand, scaling is to strong coupling as in the antiferromagnetic Kondo problem. The carriers in the two bands will bind into interband singlets, and an interband spin gap will open. The dominant response functions are then interband CDW and SS, depending on the remaining marginal intraband couplings. In this way, one can model a 1D Kondo lattice.

Pursuing the analogy of this two-band model to the Kondo problem and identifying the heavy carriers with spins, complete Kondo screening can occur because there are always sufficient light electrons to screen out the spins $\left(k_{F A}<k_{F B}\right.$ in Fig. 6.1) [218]. Caron and Bourbonnais have studied directly a 1D Kondo lattice with $n_{s}$ impurity spins and $2 n_{c}$ carriers 219. They verify by second order renormalization group Nozières' criterion [218], stating that complete Kondo screening only occurs when there are sufficient carriers $2 n_{c} \geq n_{s}$. If this criterion is violated, the spins become RKKY-coupled by the electrons

and form a $2 k_{F}-\mathrm{SDW}$, at least for weak exchange integrals. For stronger exchange, a Kondo regime may be reestablished.

Finally, we add for completeness that Emery has solved the 1D version 196 of the two-band model which was proposed in 2D for the $C u O$-high- $T_{c}$ superconductors [220]. Here, the band splitting is produced by a term in the Hamiltonian $(\epsilon / 2) \sum_{n, s}(-1)^{n} c_{n s}^{\dagger} c_{n s}$ modelling the energy difference between the copper and oxygen orbitals. In contrast to the models discussed above, the two bands do not overlap in energy, and the physics taking place upon doping is qualitatively similar to that arising from a half-filled one-band model with a charge gap, which was discussed in Section 5.2 .

\subsection{Crossover to higher dimensions}

On a macroscopic level, 1D systems are well known for their opposition to long-range order. At finite temperature, the entropy associated with the defects in an ordered phase 
more than outweighs the cost in energy for their creation [221, and thermal fluctuations thus destroy ordered phases. At $T=0$, the influence of quantum fluctuations is more subtle. From the equivalence of 1D quantum systems to 2D classical statistical mechanics, the Mermin-Wagner theorem [146] suggests that phases where a continuous symmetry would be broken, could not possess long-range order even at zero temperature. That quantum fluctuations indeed destroy long-range order associated with continuous broken symmetries was demonstrated by Takada [147]. Finite temperature phase transitions therefore must be a consequence of 3D coupling between the chains. Two mechanisms can couple electrons on different chains: finite-range Coulomb interactions and interchain tunneling.

The electron dynamics must also be affected by 3D tunneling. Assume that we have a Luttinger model on-chain dispersion $v_{F}\left(r k-k_{F}^{1 D}\right)$ and a hopping matrix element $t_{\perp}$ between neighbouring chains. The $3 \mathrm{D}$ dispersion of the electrons then becomes

$$
\varepsilon_{r}^{3 D}(\mathbf{k})=v_{F}\left(r k-k_{F}^{1 D}\right)-2 t_{\perp} \cos \left(k_{\perp b} b\right)-2 t_{\perp} \cos \left(k_{\perp c} c\right)
$$

where $b$ and $c$ are the transverse lattice constants (the longitudinal one is denoted $a$ ). We find for the Fermi surface

$$
\mathbf{k}_{F, r}^{3 D}=\left(\begin{array}{c}
r\left[k_{F}^{1 D}+\frac{2 t_{\perp}}{v_{F}} \cos \left(k_{\perp b} b\right)+\frac{2 t_{\perp}}{v_{F}} \cos \left(k_{\perp c} c\right)\right] \\
\mathbf{k}_{\perp}
\end{array}\right) .
$$

If $t_{\perp}$ is of the order of $t_{\|} \sim v_{F} / a$, the Fermi surface will be closed and we better start from an anisotropic 3D system. For smaller $t_{\perp}$, however, the Fermi surface consists of two warped sheets, Fig. 6.2, and retains some $1 \mathrm{D}$ character. The issue now is the transverse coherence of the electronic motion. Naively, we expect that at high temperatures $T>t_{\perp}$, where there is "thermal blurring" of the Fermi surface of the order $T$, the electrons are unable to sense the warping and behave essentially 1D. At $T<t_{\perp}$, the warping and thus the 3D aspects of the Fermi surface can be probed, and transverse coherence would emerge. We shall see below that the actual picture is significantly more complicated.

The reasons for these complications reside in the electron-electron interaction which may possibly confine electrons on their chains. In 1D, here are two dramatic differences from the free particle picture implictly assumed in the preceding arguments: the vanishing quasi-particle residue at the Fermi energy, and charge-spin separation. $t_{\perp}$ transfers particles or at least quasi-particles. Its efficiency may therefore be severly reduced if there are only collective excitations. While $z\left(k=t_{\perp} / v_{F}\right) \neq 0$ in general, we still expect that a reduced quasi-particle residue $z\left(t_{\perp}\right) \ll 1$ will delay the establishment of 3D coherence in the single-electron dynamics [21, 22, 222]. Charge-spin separation could also confine particles on their chains because a holon and spinon must tunnel together - yet in general they are separated [223].

The theory we have outlined in the preceding paragraphs relies heavily on the 1D nature of our models. The key point were the 1D conservation laws of charge and spin currents, i.e. of charge and spin on each branch of the dispersion separately, Eq. (3.7) which no longer hold at $t_{\perp} \neq 0$. Is the theory of the preceding chapters therefore limited 
to strictly one dimension, or can it be extended beyond? What is its relevance for highly anisotropic quasi-1D problems? Can it provide a framework to describe the physics of real quasi-1D materials such as the organic (super-)conductors?

To answer this questions, we consider an array of coupled chains to mimic a 2D or 3D situation. The Hamiltonian then becomes

$$
\begin{aligned}
H & =H_{\|}+H_{\perp}^{(t)}+H_{\perp}^{(g)} \\
H_{\|} & =\sum_{n} H_{n}^{\|}, \\
H_{\perp}^{(t)} & =-t_{\perp} \sum_{<m, n>, r, s} \int d x \Psi_{m, r, s}^{\dagger}(x) \Psi_{n, r, s}(x)+\text { H.c. } \\
& =-2 t_{\perp} \sum_{k \approx r k_{F}, \mathbf{k}_{\perp}, r, s}\left[\cos \left(k_{\perp b} b\right)+\cos \left(k_{\perp c} c\right)\right] c_{k, \mathbf{k}_{\perp}, r, s}^{\dagger} c_{k, \mathbf{k}_{\perp}, r, s}, \\
H_{\perp}^{(g)} & =\frac{2}{L} \sum_{k, \mathbf{k}_{\perp}} g_{2}^{(\perp)}\left(k, \mathbf{k}_{\perp}\right) \rho_{+}\left(k, \mathbf{k}_{\perp}\right) \rho_{-}\left(-k,-k_{\perp}\right)+ \\
& +\frac{1}{L} \sum_{k, \mathbf{k}_{\perp}, r} g_{4}^{(\perp)}\left(k, \mathbf{k}_{\perp}\right) \rho_{r}\left(k, \mathbf{k}_{\perp}\right) \rho_{r}\left(-k,-k_{\perp}\right)+ \\
& +\sum_{<m, n>, s, s^{\prime}} g_{1, m, n}^{(\perp)} \int d x \Psi_{m,+, s}^{\dagger}(x) \Psi_{n,-, s^{\prime}}^{\dagger}(x) \Psi_{n,+, s^{\prime}}(x) \Psi_{m,-, s}(x)
\end{aligned}
$$

Here, $H_{n}^{\|}$is the one-chain Luttinger Hamiltonian (3.1) for chain $n$ where the density operators acquire an additional chain $(n)$ or transverse momentum $\left(\mathbf{k}_{\perp}\right)$ label. $t_{\perp}$ is the transverse hopping integral, $m, n$ in the sums denotes the chains, and $r$ and $s$ are the branch and spin index, respectively. We have allowed for different lattice constants in all three directions. $g_{2}^{(\perp)}$ and $g_{4}^{(\perp)}$ are the interchain forward scattering constants of $g_{2^{-}}$and $g_{4}$-type, respectively, which, in the case of a Coulomb interaction, must be equal. $g_{1}^{(\perp)}$ measures the strength of the interchain backscattering. If this term is included into the Hamiltonian, consistency would require that one include also the intrachain backscattering Hamiltonian (4.6) into $H_{n}^{\|}$, in order to treat both interactions on an equal footing.

We first discuss the interchain Coulomb interaction $\left(t_{\perp}=0\right)$. Quite generally, transverse Coulomb coupling screens the effective on-chain interactions or, if negative initially, makes them more negative [224, 225, 226] - a tendency reminiscent of Little's old suggestion in favour of quasi-1D materials as candidates for high-temperature superconductivity [227. However, a more detailed investigation leads to rather different conclusions. Interchain forward scattering $\left(g_{2}^{(\perp)}, g_{4}^{(\perp)}\right)$ respects the conservation of total charge and spin on each branch of the dispersion on each chain separately. Moreover, $g_{2}^{(\perp)}$ is a marginal operator and only couples the charge fluctuations; therefore it will influence the dimensions of operators, and the exponents of those correlation functions which are sensitive to charge fluctuations. The Hamiltonian can be diagonalized exactly, and one obtains renormalized values of $K_{\rho}\left(\mathbf{k}_{\perp}\right)$ depending now on the perpendicular wavevector $\mathbf{k}_{\perp}$ [224, 225, 226]. Exponents of on-chain correlation functions then contain integrals over $\mathbf{k}_{\perp}$ involving $K_{\rho}\left(\mathbf{k}_{\perp}\right)$ or functions thereof while the spin parts can be taken over unchanged from the singlechain problem. Physically, the system remains a Luttinger liquid but, concerning the competition between SS and CDWs, SS is favoured at the expense of CDWs, not unlike 
Little's suggestion [227. The situation is completely different if interchain backscattering $\left(g_{1}^{(\perp)}\right)$ is allowed. Firstly, as on a single chain, it violates the separate conservation of total spin on each dispersion branch. But, unlike the intrachain backscattering, (6.25) also violates separate charge conservation. This indicates that, if this interaction process can become relevant, gaps may open in the charge and spin fluctuations with the concomitant possibility of long-range CDW order. This is indeed what happens, at least in the regime of attractive on-chain backscattering, but also for repulsive backscattering if the complete intra- and inter-chain potential has $\delta$-function shape [224, 225, 226]. Depending on the sign of $g_{1}^{(\perp)}$, long-range CDW order can be stabilized with a wavevector $Q_{<}=\left(2 k_{F}, 0,0\right)$ (i.e. a CDW in phase on neighbouring chains) for $g_{1}^{(\perp)}<0$, and $Q_{>}=\left(2 k_{F}, \pi / b, \pi / c\right)$ (i.e. the CDWs on neighbouring chains are out of phase) for $g_{1}^{(\perp)}>0$. Physically, these results are quite easy to understand for dominant on-chain-CDW fluctuations because the system can gain Coulomb energy from the charge modulations on the neighbouring chains with $Q_{<}$resp. $Q_{>}$.

Up to this point, we have allowed general coupling contants $g_{i}$ both for the intra- and interchain interactions. Of course, the physical Coulomb potential is $V(\mathbf{q})=4 \pi e^{2} / \mathbf{q}^{2}$, and finite forward scattering constants only arise as a consquence of screening ( $g_{1}$ can be considered as constant but may depend on details of the wavefunctions of particular materials). The problem of screening of the divergence of the Coulomb potential can be solved on an array of chains [52, 228]: the interaction of two electrons on a given chain can be screened by the electrons on the others.

The Hamiltonian

$$
H_{C}=\frac{e^{2}}{2} \sum_{n, n^{\prime}} \int d z d z^{\prime} \frac{\rho_{n}(z) \rho_{n^{\prime}}\left(z^{\prime}\right)}{\sqrt{a^{2}\left[\left(n_{x}-n_{x}^{\prime}\right)^{2}+\left(n_{y}-n_{y}^{\prime}\right)^{2}\right]+\left(z-z^{\prime}\right)}}
$$

[where $e$ is the electron charge, $\rho_{n}(z)$ the total charge density operator (3.44) at position $z$ on chain $n$, and $a$ the transverse lattice constant] can be mapped onto the form (3.25) with the couplings

$$
g_{2 \rho}(\mathbf{q})=g_{4 \rho}(\mathbf{q})=\frac{4 \pi e^{2}}{a^{2}\left(\varepsilon_{\|} q_{z}^{2}+\varepsilon_{\perp} q_{\perp}^{2}\right)} .
$$

(In principle, there is an infinite sum over transverse reciprocal lattice vectors, but the matrix elements then will depend again on details of wavefunctions and are not universal [52]). $\varepsilon_{\|, \perp}$ are background dielectric constants. The $g_{i \sigma}$ remain unaffected. One now can diagonalize the Hamiltonian for each $\mathbf{q}_{\perp}$ leading to $\mathbf{q}_{\perp}$-dependent velocities and coupling constants. The energy of the charge excitations is

$$
\omega_{\rho}(\mathbf{q})=\sqrt{v_{F}^{2} q_{z}^{2}+\frac{\omega_{p l}^{2} q_{z}^{2}}{\varepsilon_{\|} q_{z}^{2}+\varepsilon_{\perp} \mathbf{q}_{\perp}^{2}}} \quad, \quad \omega_{p l}^{2}=\frac{8 e^{2} v_{F}}{a^{2}}=\frac{4 \pi e^{2} n}{m} .
$$

For any finite $\mathbf{q}_{\perp}, \omega_{\rho}\left(q_{z}\right) \propto\left|q_{z}\right|$ in the limit $q_{z} \rightarrow 0$, allowing to define a renormalized charge velocity $v_{\rho}\left(\mathbf{q}_{\perp}\right)$. For $|\mathbf{q}| \rightarrow 0$, one obtains the plasma frequency of the anisotropic system $\left(\varepsilon_{\|}=\varepsilon_{\perp}=\varepsilon\right.$ for simplicity)

$$
\omega_{\rho}(\Theta)=\frac{\omega_{p l}}{\sqrt{\varepsilon}}|\cos \Theta|
$$


$\Theta$ is the angle between $\mathbf{q}$ and the $z$-axis. A renormalized coupling constant $K_{\rho}\left(\mathbf{q}_{\perp}\right)$ can be defined from the diagonalization of the Hamiltonian. Its usefulness for determining the asymptotic decay of correlation functions is, however, not as immediate as for the single-chain problem. In fact, when calculating the correlation functions, one obtains $\mathbf{q}_{\perp^{-}}$ dependent expressions for their decay exponents which have to be integrated over $\mathbf{q}_{\perp} a t$ the end. It is not allowed to use an integrated $\int d^{2} q_{\perp} K_{\rho}\left(\mathbf{q}_{\perp}\right)$ in the standard Luttinger expressions. As a consequence, the simple scaling relations between the exponents of the various correlation functions (single-particle Green function, SDW, CDW, SS, ... ) break down! Each function has its own, independent exponent.

The screening effect strongly depends on the density of carriers and on the anisotropy of the lattice. Denser chain packing means better screened interaction. In the limit of vanishing packing density, one crosses over to the single-chain case [90] with a vanishing plasma frequency, a correction $\propto \ln \left|q_{z}\right|$ to the Fermi velocity, and a formally vanishing $K_{\rho}$-exponent, as discussed at the end of Section 4.4.3.

Interchain single-particle tunneling $t_{\perp}$ can lead to further new physics. $t_{\perp}$ can generate transverse coherence in the electron dynamics, i.e. a crossover from essentially $1 \mathrm{D}$ to effectively 3D behaviour. It is not clear at this time, if the effectively higher-dimensional behaviour is necessarily of Fermi-liquid type or not. Interchain tunneling also can generate transverse pair tunneling (either of particle-particle or particle-hole type) which propagate the dominant on-chain correlations in the transverse directions, and eventually a finitetemperature phase transition into a symmetry-broken ground state occurs. In both cases, the 1D Luttinger liquid is unstable.

We now consider the Hamiltonian (6.21) with $H_{\perp}^{(g)} \equiv 0$ (6.24). The essential qualitative physics can be seen from a scaling argument due to Schulz [42] and Wen [229]. Consider the free energy of a system with small $t_{\perp}$ at finite temperature and investigate the relevance of different terms generated by an expansion in $t_{\perp}$. The free energy of the strictly 1D system is $F^{(0)} \propto T^{2}$. In second order in $t_{\perp}$, we obtain a correction

$$
\delta F^{(2)} \approx t_{\perp}^{2} \int d x d \tau G_{r s}^{2}(x, \tau)
$$

where $G_{r s}(x, \tau)$ is the single-chain Green function at imaginary time $\tau$. This function behaves as

$$
\begin{aligned}
G_{r s}\left(x_{1}-x_{2}, \tau_{1}-\tau_{2}\right) & \approx|1-2|^{-1-\alpha} \\
\text { with }|1-2| & \equiv \frac{v_{F}}{2 \pi T} \sqrt{\cosh \left[2 \pi T \frac{x_{1}-x_{2}}{v_{F}}\right]-\cos \left[2 \pi T\left(\tau_{1}-\tau_{2}\right)\right]}
\end{aligned}
$$

$\alpha$ is the single-particle exponent from Section (3.3). The correction to the free energy then scales as

$$
\delta F^{(2)} \propto t_{\perp}^{2} T^{2 \alpha}
$$

If $\alpha<1$ (i.e. $3-\sqrt{8}<K_{\rho}<3+\sqrt{8}$ ), a case encountered in many models (Section 4.4), this terms will become more important than $F^{(0)}$ at sufficiently low temperature no matter how small $t_{\perp}$, indicating that $t_{\perp}$ then is a relevant perturbation. If $t_{\perp}$ is the most relevant perturbation, we expect the system to show a single-particle $1 \mathrm{D}-3 \mathrm{D}$ crossover at 
some temperature $T_{X}^{1}$. Only if $\alpha>1$ interchain single-particle tunneling will be irrelevant. But then, look at the next order in the expansion of the free energy, corresponding to interchain pair tunneling

$$
\begin{aligned}
\delta F^{(4)} & \approx t_{\perp}^{4} \int d 1 d 2 d 3 d 4\left[\frac{|1-3||2-4|}{|1-2||3-4|}\right]^{\left(K_{\rho}-1 / K_{\rho}\right) / 2}[|1-4 \| 2-3|]^{-2-2 \alpha} \\
& \approx t_{\perp}^{4} \max \left(T^{4 \alpha}, T^{2 K_{\rho}}, T^{2 / K_{\rho}}\right) .
\end{aligned}
$$

The first term corresponds to two uncorrelated single-particle events and is the square o $\delta F^{(2)}$. The second term is generated from coherent tunneling of a particle-hole pair and is more important than $F^{(0)}$ whenever $K_{\rho}<1$, i.e. for repulsive interactions. Coherent pair tunneling generates the third term which dominates the zero-order term for attractive interactions. The particle-particle and particle-hole pair terms are more important than the single-particle terms for $1 / K_{\rho}$ resp. $K_{\rho}<(2 / \sqrt{3}-1)$ i.e. rather strong interactions. If this happens, one expects to find a two-particle 1D-3D crossover at a temperature $T_{X}^{2}>T_{X}^{1}$, and the system very likely will undergo a symmetry-breaking phase transition to a ground state corresponding to the most dominant intrachain fluctuation. For spinless fermions, single-particle tunneling is relevant for $\alpha<1 / 2$ i.e. $K$ resp. $1 / K<2+\sqrt{3}$, but two-particle tunneling is stronger than single-particle tunneling already for $K$ resp. $1 / K>1+\sqrt{2}$ [230.

This renormalization group argument is good for infinitesimal transverse coupling only. It will certainly fail for bigger $t_{\perp}$ : one expects the warping of the Fermi surface and deviations from perfect nesting to cut off the Peierls divergence. Below the temperature where this cutoff happens, superconductivity in general will be the only possible instability remaining. A description of the system for finite $t_{\perp}$ is, however, a difficult task. We only briefly review the major achievements and refer the reader to more extended treatments [21, 22, 222] for further details.

Early work starts from the exact solution of the $1 \mathrm{D}$ models and adds $t_{\perp}$ as a perturbation [222, 224, 231]. In this way, one generates an effective transfer of a pair of particles. There are no real interchain single-particle transitions, and the pair motion takes place through virtual events. The perturbation theory only becomes well defined if there is either a gap in the charge or spin fluctuations, or if the Luttinger model interactions are sufficiently strong [basically, the integral in Eq. (6.30) must converge]. This is a good assumption for attractive backscattering where we have a spin gap, but not for the generic repulsive Luttinger liquid. Doing then mean-field theory in $t_{\perp}$ with spin gap, one finds a transition to a SS or CDW phase at a finite critical temperature [224]. When only one type of fluctuation is divergent on a single chain, tunneling will stabilize it into an ordered phase. When both are divergent, tunneling will favour SS.

The question of a single-particle crossover from 1D to 3D behaviour was addressed by Prigodin and Firsov [222]. When the 1D interactions are weak, the system will behave as a 1D Luttinger liquid at higher energies. Naively, one would expect a crossover to 3D behaviour at an energy of the order of the transverse bandwidth $t_{\perp} . t_{\perp}$ is, however, renormalized by the $1 \mathrm{D}$ intrachain correlations, and the $1 \mathrm{D}-3 \mathrm{D}$ crossover will only take 
place at a temperature $T_{X}^{1} \approx t_{\perp}^{\prime} / \pi$ determined by the renormalized $t_{\perp}^{\prime}$ which can be significantly lower than $t_{\perp} \cdot t_{\perp}^{\prime}$ is determined self-consistently from the requirement $t_{\perp}^{\prime}=t_{\perp} z\left(t_{\perp}^{\prime}\right)$ where $z\left(t_{\perp}^{\prime}\right)$ is the quasi-particle residue at the energy scale $t_{\perp}^{\prime}$. The renormalization of interchain tunneling $t_{\perp} \rightarrow t_{\perp}^{\prime}$ indicates a tendency of the electrons towards confinement on the chains induced by their on-chain correlations. Below the crossover temperature, the interference between the Peierls and Cooper channels is destroyed. The transition temperatures to a symmetry-broken ground state and the competition of various types of order then can be determined from standard summation of ladder diagrams. In the 1D high-energy regime, the perturbative treatment does not allow for generation of interchain pair tunneling. This, again, could only take place in the case of strong interactions or presence of a spin or charge gap, a problem that also is present in later work by Brazovskii and Yakovenko 231. Interestingly, however, in such a gapped regime, Prigodin and Firsov obtain a maximum of the superconducting $T_{c}$ as a function of $t_{\perp}$ for $t_{\perp} \sim \Delta$, where $\Delta$ is the $1 \mathrm{D}$ spin or charge gap [222]. $T_{c}$ at maximum is significantly higher than in the $3 \mathrm{D}$ limit $t_{\perp} \rightarrow t_{\|}$. The reasons for this become apparent upon realizing that the maximal $T_{c}$ just occurs at the point where the Peierls state breaks down: here one has an optimal combination of $1 \mathrm{D}$ effects (phonon softening at $2 k_{F}$ and high density of states at $\left.E_{F}\right)$ with the 3D tunneling necessary to establish superconductivity.

Bourbonnais and Caron proposed a renormalization group scheme both for the onchain interactions $g_{i}$ and the interchain tunneling $t_{\perp}$ with respect to the free Fermi gas which generates interchain pair tunneling from single particle tunneling even at weak coupling [22, 232]. The basic mechanism is shown in Fig. 6.3, where we display an expansion of the vertex corrections in terms of the $g_{i}$ and $t_{\perp}$. In particular, the last diagram corresponds to the coherent hopping of a pair to neighbouring chains. This scheme produces all kinds of pair tunneling processes because the general diagrammatic structure of Fig. 6.3 applies to all combinations of propagation directions and spins. The renormalization group transformations under a scaling of the bandwidth cutoff from $E_{0}$ to $E_{0}(\ell)$ generate terms of the type

$$
H_{\text {pair }}=\frac{1}{4} \sum_{\mu, i, j, q, \omega_{n}} V_{\mu}(\ell) O_{\mu, i}^{\dagger}\left(q, \omega_{n}\right) O_{\mu, j}\left(q, \omega_{n}\right),
$$

where finite pair tunneling matrix elements $V_{\mu}$ arise from $t_{\perp}$ through

$$
\begin{aligned}
\frac{d V_{\mu}\left(\mathbf{k}_{\perp}\right)}{d \ell} & =f_{\mu}(\ell)\left[\cos \left(k_{\perp b} b\right)+\cos \left(k_{\perp c} c\right)\right]+V_{\mu}\left(\mathbf{k}_{\perp}, \ell\right) \frac{d \ln \bar{X}_{\mu}(\ell)}{d \ell}-\frac{\left[V_{\mu}\left(\mathbf{k}_{\perp}, \ell\right)\right]^{2}}{2 \pi v_{F}}(6.35) \\
f_{\mu} & = \pm 2 \pi v_{F}\left(\frac{t_{\perp}^{\prime}}{E_{0}(\ell)}\right)^{2} g_{\mu}^{2}(\ell) .
\end{aligned}
$$

Here, the index $\mu=\mathrm{CDW}$,SDW,SS,TS denotes the different kinds of fluctuations, the operators $O_{\mu, i}$ describe these fluctuations on chain $i$ as in Eqs. (3.89) or (3.94)-(3.96), and the $g_{\mu}$ denote effective combinations of the coupling constants $g_{i}$ relevant for the respective operators. In (6.36), the plus-sign applies for $\mu=\mathrm{CDW}, \mathrm{SDW}$ and the minussign for $\mu=$ SS,TS. $\bar{X}_{\mu}(\ell)$ is an auxiliary pair correlation function for fluctuations of type $\mu$. The last term is an RPA-like interchain ladder contribution, the second term is the 
pair vertex correction whose strong-coupling limit basically has been treated in the earlier work, and the first term generates finite $V_{\mu}$ from the initial value $V_{\mu}=0$.

The fluctuation $\mu$ has a tendency to long-range order at that wavevector $\mathbf{k}_{\perp}$ for which $V_{\mu}\left(\mathbf{k}_{\perp}\right)$ is negative and extremal. One now integrates the renormalization group equations (approximately) and finds the effective pair-tunneling amplitudes in all situations of interest, both for weak and strong coupling, and on high and low energy scales. Interestingly, the solutions allow for a regime where, for weak on-chain interactions, scaling of $V_{\mu}$ goes to strong coupling at energies above $T_{X}^{1}$. This corresponds to the growth of critical fluctuations of type $\mu$ gaining 3D coherence. There can thus be a two-particle $1 \mathrm{D}-3 \mathrm{D}$ crossover temperature $T_{X}^{2}>T_{X}^{1}$ where interchain pair tunneling becomes coherent despite essentially 1D single-particle dynamics. This complements, at weak coupling, earlier work in the strong-coupling or gapped regime 231]. Moreover, since the interchain interactions strongly depend on temperature, they can change from repulsive at high temperature to attractive at low temperature. In this case, one will find antiferromagnetic fluctuations coexisting with singlet superconductivity. At lower temperatures $T<T_{X}^{1}$, in the absence of perfect nesting, a transition to superconductivity occurs. Here, interchain Cooper pairs form, and the superconducting gap has a line of zeros on the Fermi surface. By combining the quasi-1D renormalization group with approaches like RPA and parquet summation, a variety of useful results on critical temperatures and response functions in the presence of interchain tunneling can be computed in all relevant regimes [22].

By 1991, it was believed that this series of work provided quite detailed a picture for the crossover from one into three dimensions both concerning the electron dynamics and the establishment of long-range order of some symmetry-broken phase. Then, Anderson pointed out that both our simple renormalization group argument at the beginning of this section as well as the series of detailed calculations reviewed thereafter, are irrelevant because they neglect charge-spin separation [223]. This is supposed to be a particularly serious flaw because charge-spin separation is a consequence of the restricted phase space in $1 \mathrm{D}$ and a nonperturbative effect. According to Anderson, there is a well-defined order in which to turn on interactions and $t_{\perp}$ : interactions first, and then $t_{\perp}$. In this way, electrons on the chains would first separate into holons and spinons and inactivate $t_{\perp}$, because it requires both of them, i.e. an electron, to tunnel. Consequently, the electrons would be confined to a 1D chain. Only pair tunneling of holons and spinons would be allowed, and therefore, in the language of the preceding paragraphs, one would necessarily have a two-particle 1D-3D crossover. A single-particle crossover, presumably to a Fermiliquid state, would be forbidden. In addition, anomalous fermion dimensions could, of course, strengthen the intrachain confinement [233]. Anderson also pointed out that two chains are enough to study confinement which introduces considerable simplification in the actual calculations.

Anderson's suggestion has spun off a flurry of activity on two-chain Hubbard and Luttinger models. Many of these do not follow Anderson's prescription and diagonalize the bandstructure first and then turn on the interactions. Moreover, charge-spin separation is often neglected, again. In this way, one arrives at the effective two-band models discussed before. 
Charge-spin separation is present in a one-branch Luttinger liquid where the only allowed interaction is $g_{4 \perp} \neq g_{4 \|}$. The question "confinement or not?" can be studied here on a minimal model. Fabrizio and Parola have produced an exact solution of such a two-chain one-branch Luttinger liquid following precisely Anderson's prescription in that they first produce charge-spin separation and then turn on $t_{\perp}$ [234, 235]. The Hamiltonian is (6.23) with (3.2) and (3.4) for each chain. Keeping only the right-moving particles and dropping the corresponding index $r=+$ on the operators,

$$
\begin{aligned}
H & =\frac{2 \pi}{L} v_{\rho} \sum_{p>0}\left[\rho_{1}(p) \rho_{1}(-p)+\rho_{2}(p) \rho_{2}(-p)\right] \\
& +\frac{2 \pi}{L} v_{\sigma} \sum_{p>0}\left[\sigma_{1}(p) \sigma_{1}(-p)+\sigma_{2}(p) \sigma_{2}(-p)\right]-t_{\perp} \sum_{k, s}\left[c_{k s 1}^{\dagger} c_{k s 2}+\text { H.c. }\right] .
\end{aligned}
$$

The index 1,2 labels the chains. The on-chain part of the Hamiltonian has already been diagonalized and exhibits charge-spin separation $v_{\rho} \neq v_{\sigma}$. We now can use the bosonization identity (3.41) for the interchain part $H_{\perp}$ in order to get its representation in terms of the on-chain phase fields $\Phi_{\nu}$ and $\Theta_{\nu}$, Eqs. (3.42) and (3.43). Introducing symmetric and antisymmetric combinations of the charge and spin density operators $\nu_{1,2}(p)$ of the different chains, the resulting boson Hamiltonian can be refermionized simply by inverting the bosonization identity (3.41). Then going through a series of unitary transformations, one finally obtains a Hamiltonian bilinear in fermions with four dispersion branches

$$
\begin{array}{ll}
\epsilon_{1}(q)=v_{\rho} q & \epsilon_{3}(q)=\frac{\left(v_{\rho}+v_{\sigma}\right) q}{2}+\sqrt{\left[\frac{\left(v_{\rho}-v_{\sigma}\right) q}{2}\right]^{2}+4 t_{\perp}^{2}} \\
\epsilon_{2}(q)=v_{\sigma} q & \epsilon_{4}(q)=\frac{\left(v_{\rho}+v_{\sigma}\right) q}{2}-\sqrt{\left[\frac{\left(v_{\rho}-v_{\sigma}\right) q}{2}\right]^{2}+4 t_{\perp}^{2}} .
\end{array}
$$

A particle-hole transformation having been performed in the course of the calculation, these expressions are only defined for $q>0$.

Two excitation branches 1,2 retain the original Luttinger dispersion: they originate from the chain-symmetric linear combinations of the density operators which remains unaffected by $t_{\perp}$. The antisymmetric combinations are shifted by $t_{\perp}$. At $q=0$, they are split from the Fermi level by $\pm 2 t_{\perp}$ and for $q \rightarrow \infty$, they approach the two Luttinger branches: $\epsilon_{3,4} \rightarrow v_{\nu} q$. The ground state of the system is thus obtained by occupying the branch 4 up to $Q=2 t_{\perp} / \sqrt{v_{\rho} v_{\sigma}}$, i.e. up to $\epsilon_{4}(Q)=0$, the chemical potential. Information on the confinement of the carriers on individual chains can be obtained from several quantities. The ground state energy change due to $t_{\perp}$ is determined by the occupation of the branch 4

$$
\frac{\Delta E}{L}=-\frac{1}{2 \pi} \frac{4 t_{\perp}^{2}}{v_{\rho}-v_{\sigma}} \log \left(\frac{v_{\sigma}}{v_{\rho}}\right) .
$$

Being of order $t_{\perp}^{2}$, it indicates that there is a finite ground state expectation value of $H_{\perp}^{(t)}$ (e.g. $\Delta E \propto t_{\perp}^{2}$ is obtained for free particles where $t_{\perp}$ shifts the bands). The occupation number difference between the bonding and anti-bonding bands (labelled by their $k_{\perp^{-}}$ values 0 and $\pi$ ) is

$$
\frac{\left\langle N_{0}-N_{\pi}\right\rangle}{L}=\frac{4 t_{\perp}}{2 \pi} \frac{1}{v_{\rho}-v_{\sigma}} \log \left(\frac{v_{\sigma}}{v_{\rho}}\right)
$$


also indicating a shift between the bonding and anti-bonding bands $\propto t_{\perp}$. The difference in occupation numbers corresponds to a shift of the Fermi wavevector between the bonding and antibonding bands of $2 \Delta k_{F}$ with

$$
\Delta k_{F}=\frac{t_{\perp}}{v_{\rho}-v_{\sigma}} \log \left(\frac{v_{\rho}}{v_{\sigma}}\right) .
$$

All three quantities show that interchain tunneling does shift the bonding with respect to the antibonding band, and that there is no confinement of electrons on individual chains despite charge-spin separation. On the other hand, the nature of the spectrum indicates that charge-spin separation is a phenomenon robust against interchain coupling so that it could conceivably survive under certain circumstances in more than one dimension, and with it some Luttinger liquid physics.

This is made more clear in the single- and many-particle dynamics. The Green function for particles with transverse momentum 0 or $\pi$ can be calculated from a cumulant expansion and becomes

$$
\left\langle\Psi_{0, \pi}(x, t) \Psi_{0, \pi}^{\dagger}(0,0)\right\rangle \sim e^{i\left(k_{F} \pm \Delta k_{F}\right) x}\left(x-v_{\rho} t\right)^{-3 / 8}\left(x-v_{\sigma} t\right)^{-3 / 8}\left(x-v_{r} t\right)^{-1 / 4}
$$

where $v_{r}=2 v_{\rho} v_{\sigma} /\left(v_{\rho}+v_{\sigma}\right)$ and the $+(-)$-signs go with $k_{\perp}=0, \pi$, respectively. The corresponding spectral function still is purely incoherent - there is no quasi-particle-like feature - but now, it exhibits a three-peak structure at wavevectors small with respect to $t_{\perp} /\left(v_{\rho}-v_{\sigma}\right)$, compared to two peaks for the isolated chains. In the new excitation dispersing with $v_{r}$, charge and spin strongly interact through $t_{\perp}$. At large wavevectors, on the contrary, $t_{\perp}$ seems to be inefficient, and the spectral function reduces to that of two uncoupled chains [53, 59, 61]. The long-wavelength charge and spin density correlation functions are also changed. The on-chain spectral function for the charge fluctuations contains a pole contribution from the Luttinger branch which has the form of isolated chains. In addition, there are incoherent pieces close to $v_{\rho} q \pm 2 t_{\perp}$ indicating branch cuts in the correlation function, which originate from the coupling between charge and spin fluctuations generated by $t_{\perp}$ as well as the nonlinear dispersion of $\epsilon_{3,4}(q)$. As $q$ is increased, spectral weight is transferred from the incoherent features into the central pole.

For the special problem of the two-chain-one-branch Luttinger liquid, the same results are found by proceeding in the opposite sense: first diagonalize the band structure and then turn on the interactions [236]. Here, one bosonizes the bonding and antibonding fermions, but now the antiparallel-spin interactions lead to a Hamiltonian which is highly nonlinear in the boson operators [of the structure of the backscattering Hamiltonian $H_{1 \perp}$ in Eq. (4.6) but with right- or left-moving fields only], so that an exact solution no longer is feasible. Still, the Hamiltonian separates into four pieces corresponding to the four excitations found in Eq. (6.38). These spectra can be determined from thermodynamics and using special symmetries of the model, and agree with (6.38). The Green function then obtains as Eq. (6.42).

Although obtained from an exceedingly simple Hamiltonian, these results are extremely important. They tell us (i) that even in the case where the interchain tunneling is turned on after the establishment of charge-spin separation on the chains, there is 
no confinement of electrons by this special kind of 1D interactions, and their interchain dynamics can become coherent; (ii) the results do not depend on the order of turning on charge-spin separation and interactions. Since charge-spin separation was the only important feature left out in the work reviewed at the beginning of this section, we get additional confidence in the relevance of its conclusions.

One can now include the $g_{2}$-forward scattering into a two-chain model. This couples right-and left-moving electrons and gives rise to the anomalous dimensions on a single chain parameterized by $K_{\rho}$. On a more qualitative level, one can study confinement by looking at the stability of the 1D momentum distribution function $n(k)$, Eq. (3.86), with respect to $t_{\perp}$. Turning on $t_{\perp}$ at the end, one finds [237]

$$
\delta n(\mathbf{k})= \begin{cases}t_{\perp} \cos k_{\perp}\left[C+D\left(k_{\|}-k_{F}\right)^{2 \alpha-1}\right] & \text { for } \quad \alpha<1 \\ t_{\perp} \cos k_{\perp}\left[C+D\left(k_{\|}-k_{F}\right)\right] & \text { for } \quad \alpha \geq 1 .\end{cases}
$$

There is a singular correction to the momentum distribution in the neighbourhood of $k_{F}$ for small $\alpha$ while it vanishes for large $\alpha$. This essentially reproduces the conclusions of our scaling argument on the level $\delta F^{(2)}$ from the beginning of this section, Eq. (6.32). Of course, in the large- $\alpha$ limit, we will find relevant pair-tunneling processes which are not covered by this argument.

A more detailed analysis is possible if one accepts first diagonalizing the band structure - but from the behaviour of the two-chain-one-branch model above, we expect this procedure to be safe. In a renormalization group analysis of Luttinger models $\left(g_{2}, g_{4} \neq 0\right)$ coupled by $t_{\perp}$, new relevant interactions are generated by the RG 236]. Specifically, an interaction term

$$
H_{\lambda}=\frac{\lambda}{4 L} \sum_{|p| \ll k_{F}}\left[\rho_{+, 0}(p)-\rho_{+, \pi}(p)\right]\left[\rho_{-, 0}(p)-\rho_{-, \pi}(-p)\right]
$$

is generated and goes relevant, and its coupling constant $\lambda$ increasing towards large negative values under renormalization - independent of the sign of the on-chain $g_{2}$ ! It is driven by interband forward scattering with antiparallel spins involving opposite branches (i.e. of interband- $g_{2}$-type but formally of structure similar to the usual backscattering Hamiltonian) which does not conserve the total spin on each of the four excitation branches. In Eq. (6.44), $\rho_{r, k_{\perp}}(p)$ denotes the right- or left-moving $(r=+,-)$ density fluctuations obtained from the bonding or antibonding $\left(k_{\perp}=0, \pi\right)$ fermions with parallel momentum $p$. Due to the shift between the bands brought about by $t_{\perp}$, the differences in [...] do not equal the on-chain densities. One can now bosonize the strong-coupling fixed point and find that a spin gap opens in the system. At this level, it is not completely clear if this favours SS or CDW correlations, but in a 3D array of pairs of chains, the Peierls divergence will be cut off by $t_{\perp}$ and superconductivity comes up [236]. Here, the Cooper pairs can be formed predominantly either on or between the chains, depending on the sign of $g_{2}$. For repulsive interactions, one would find pairing between the chains, in qualitative agreement with Bourbonnais and Caron [22].

One can go one step further and consider the Hubbard model on two-chains - finally, this is the problem we are most interested in! This requires the inclusion of all kind 
of backscattering processes, and one has to treat a problem with fifteen independent coupling constants (excluding commensurate situations where additional Umklapps come up) [235, 238]. The phase diagram is given in Fig. 6.4. There is a trivial Luttinger liquid (LL1) at large $t_{\perp}$ where the upper band is empty and only the bonding band is filled. When the two bands only slightly overlap, there is another Luttinger liquid (LL2) whose existence is related to a big difference between the bonding and antibonding Fermi velocities. However, there is a finite value of $t_{\perp}^{\text {eff }}$ and no confinement. Decreasing $t_{\perp}$, one enters strong-coupling phases. The phase III at large $U$ and small $t_{\perp}$ does have confinement $\left(t_{\perp}^{\text {eff }}=0\right)$ and strong pair hopping between the chains. It is interesting that an interband pair susceptibility, pairing particles of the bonding with those of the antibonding band, has the most divergent fluctuations, but on-chain SDWs diverge, too. At smaller $U$, one enters another phase I where the dominant pairing fluctuations involve pairs from the bonding or from the antibonding bands. An interchain SDW diverges, too, though less strongly. Intercalated between those phases may be a third phase II with conventional on-chain Cooper pairing.

The correlations on two Hubbard chains can also be studied with numerical calculations 239. A density-matrix renormalization group study finds a spin gap at half-filling with exponentially decaying spin-spin correlations. The correlation length is a few lattice constants. Singlet pairing correlations also decay exponentially, and their correlation length is even shorter which may indicate a liquid of disordered singlets. If one dopes the systems with holes, the spin gap is quite robust and persists down to at least $n \sim 3 / 4$ i.e. a doping level of $25 \%$. On the other hand, the doping greatly favours the pairing correlations (both on and between the chains i.e. in a d-wave like pattern) which change from exponential to power-law and therefore will be dominant at long distance. However, they decay as $1 / r^{2}$, like free fermions which is definitely weaker than the divergences predicted from renormalization group. In addition, no sign of the subdominant SDW divergences predicted by renormalization group is reported from the numerical calculations. Another Quantum Monte Carlo study where the reduced density matrix of the superconducting correlations was computed, does find evidence for enhanced superconducting correlations with respect to the uncorrelated system at $n=3 / 4$ [240]. There is some structure in this enhancement when $t_{\perp}$ is varied at fixed $U$ which has been associated with the different phases LL2, SC1, and SC2 in Fig. 6.4. When $t_{\perp}$ becomes so large that only the bonding band is occupied (LL1), no enhancement can be detected. On the other hand, a finite size analysis of the correlations assuming the Luttinger liquid power laws (3.92) and (3.99) suggests that the exponent $K_{\rho}<1$ which would imply dominant density wave and only leave space for subdominant SS correlations [240]. This conclusion does not agree with the renormalization group work 235, 238.

Much information has also been gathered on arrays of coupled $t-J$-chains following a suggestion that they could be used as a model for certain cuprate compounds 241]. Two or more chains described by the standard $t-J$-Hamiltonian (4.21) are coupled by transverse hopping $\left(t_{\perp}\right)$ and and exchange $\left(J_{\perp}\right)$ integrals. In an undoped system of two (or an even number of) coupled Heisenberg chains [242], if $J_{\perp} \gg J$, of course singlet pairs will form across the rungs of the ladder, and the excitations will have a spin gap. 
This singlet-triplet gap survives not only down to the isotropic point $J_{\perp}=J_{\|}$but there is evidence that it does so for any finite $J_{\perp}$ [242]. On the other hand, for an odd number of chains, no such dimer state is possible, and it turns out that its excitations are gapless like in the single-chain model. Introducing holes into two chains will lower but not destroy the spin gap [243, 244]. One can now combine this fact with a bosonization analysis to inquire what type of correlations will govern the physics of this two-chain system. In a spin-singlet state, the effective exponents for the charge degrees of freedom become

$$
\frac{1}{K_{\rho}^{ \pm}}=\sqrt{1+\frac{J}{\pi t}\left\langle\mathbf{S}_{i j} \cdot \mathbf{S}_{i+1 j}\right\rangle \pm \frac{J_{\perp}}{\pi t}\left\langle\mathbf{S}_{i 1} \cdot \mathbf{S}_{i 2}\right\rangle}
$$

where \pm stands for the chain-symmetric (antisymmetric) combination of the phase fields $\Phi_{\nu}$ and $\Theta_{\nu}$ used to bosonize the single chain, $\mathbf{S}_{i j}$ is the spin operator at site $i$ of chain $j=1,2$, and the expectation values do not depend on the site index $i$ [243]. In general, one has $K_{\rho}^{+}>1$ corresponding to attractive interactions in the bonding channel which are generated by the preferred singlet $\left(\left\langle\mathbf{S}_{i 1} \cdot \mathbf{S}_{i 2}\right\rangle<0\right)$ correlations across the rung of the ladder. This analysis cannot determine if $K_{\rho}^{-}>$or $<1$, which would correspond to modified dwave SS correlations or a special CDW phase where an alternating flux $\Phi=2 k_{F}$ is enclosed in a plaquette [243]. Such an "orbital antiferromagnet" had been discovered earlier in a study of a two-chain model of spinless fermions with nearest-neighbour interaction [213]. Numerical calculations seem to prefer the d-wave SS correlations 244. The orbital antiferromagnet is a two-chain version of the flux states discussed for the $2 \mathrm{D}$ high- $T_{c}$ superconductors; these flux phases have been discussed also for anisotropic 2D systems as we consider them here [245]. They model systems with both open and closed orbitals in the neighbourhood of the Fermi surface, and in the anisotropic limit, instabilities reminiscent of the $1 \mathrm{D}$ systems are found.

There is also a detailed picture of the excitations created upon hole-doping a $t-J$ ladder [244]. The lowest excitation at half-filling is a spin-triplet above the gap. Doped holes (with concentration $\delta$ ) will pair so that the spins can take advantage of the singlet binding across the rungs. One obvious magnetic excitation is the triplet, again, which can propagate in the ladder with an exchange integral $J / 2$ while the hole pair moves with $1 /\left(J_{\perp}-4 / J_{\perp}\right)$. The number of such possible triplets goes as $(1-\delta)$. But there is another possibility: one can form quasi-particles, singly occupied rungs, carrying charge and spin$1 / 2$, as a bound holon-spinon pair. These quasi-particles will move with a hopping element $t / 2$ and their number scales with $\delta$. They have triplet spin correlations. In a wide range of $J_{\perp}$, the creation of such quasi-particles is energetically favourable, and the spin gap is then reduced from the singlet-triplet gap of the half-filled model. Consequently, the dynamics and thermodynamics of the spin excitations is dominated by different energy scales as the doping level is varied: increasing doping will bring up a new low-energy scale associated with the quasi-particle excitations [244]. The quasi-particles also show up in the single-particle spectral function although there are sizable incoherent contributions. There is a coherent peak dispersing towards the Fermi energy as $k \rightarrow k_{F}$, until the spin gap is reached. In this limit, the particle peak at $\omega>0$ has acquired a strong shadow component at $\omega<0$, as in a superconductor [244]. On the other hand, this strong 
shadow component is the direct continuation of the spectral weight at $\omega<0$ found for the Luttinger liquid in Section 3.3, Fig. 3.6, to a situation where a fully developed spin gap exists.

A generalization of this picture for four coupled $t-J$-chains is now available [246]. Also, the interplay of superconductivity and phase separation has been studied in the regime of large $J / t$ [247]. Here, a strong possibility for phase separation between the chains is found, and this is precisely the range where strong signals of superconductivity are detected. Notice, however, that these $J$ values are out of the range which can be derived from large- $U$ Hubbard models, although more general models do allow them [97].

Very recently, the partition and Green functions have been derived for Luttinger liquids on an arbitrary number of chains coupled by interchain hopping, including charge-spin separation [248]. This analysis essentially confirms the earlier results [22] where this feature had been neglected. Provided the interactions are not so strong that a twoparticle crossover would occur before the single-particle crossover, this work provides us with Green functions containing explicitly a quasi-particle residue indicative of a Fermi liquid ground state in this case, plus correction terms containing the remnants of the 1D Luttinger liquid. Others give explicit spectral functions for the case of selfconsistently screened Coulomb interactions [52, 228] including interchain hopping [249] although some approximations made may overestimate the anomalous 1D component of the spectra.

An important virtue of the variational wavefunctions used for mapping out the Luttinger liquid correlations of the $1 \mathrm{D} t-J$-model in Section 4.4 is the possibility to generalize them to 2D 250]. The Luttinger liquid state with nontrivial $K_{\rho}$ is stabilized by gains in kinetic energy with respect to the Gutzwiller wave function describing a Fermi liquid. It possess the anomalous dimensions of a Luttinger liquid but no charge-spin separation. Moreover, as in the 1D case, one can apply the power method to obtain increasingly accurate approximations to the true ground state which conserve the typical power laws [251. There have been claims of charge-spin separation in the $2 \mathrm{D} t-J$-model based on high-temperature expansion [252] but this interpretation of the data has been opposed by others 253.

An approach rather different from the work above has been taken by Castellani et al. who consider fermions with short-range interactions in continuous dimensions $1 \leq D \leq$ 2 [254]. Here, the Fermi surface is closed and isotropic in the $D$-dimensional reciprocal space while the approaches coupling 1D Luttinger liquids with $t_{\perp} \ll t_{\|}$all imply open warped Fermi surfaces which conserve a strong 1D character. They find that while the 1D conservation laws for total charge and spin on a branch of the dispersion, Eqs. (3.106) and (3.107), are no longer satisfied exactly, similar laws for charge and spin associated with directions radially outward from the $D$-dimensional Fermi surface are still obeyed asymptotically. This allows the formulation of corresponding asymptotic Ward identities which strongly constrain the low-energy physics close to the Fermi surface. In particular, a Fermi liquid fixed point is found for all dimensions $D>1$. However, for dimensions $D \leq 2$, there are dramatic corrections to quasi-particle behaviour away from the fixed point. They are strongly reminiscent of the behaviour of 1D Luttinger liquids but now in radial direction. Such a "tomographic Luttinger liquid" behaviour at finite energy 
could completely mask the Fermi liquid fixed point physics, and provide a realization of Anderson's suggestion [11]. Singular interactions, as proposed by Anderson, could then conceivably stabilize such physics also at the fixed point.

We finally mention that there is a variety of work in $2 \mathrm{D}$ producing evidence for nonFermi-liquid and possibly Luttinger liquid low-energy physics using peculiar, often longrange, interaction Hamiltonians [255]. Others attempt to describe the Fermi liquid with methods borrowed from the 1D systems reviewed here, such as bosonization and renormalization group [256]. In some sense, one goes the way opposite to the one we took in Section 3.5 where we applied standard techniques of Fermi liquid theory in 1D. Further development of these methods will hopefully sharpen our understanding of scenarios for a possible breakdown of Fermi liquid theory in higher dimensions, and for similarities and differences to the $1 \mathrm{D}$ case reviewed here.

\subsection{Edge states in the quantum Hall effect}

When a 2D electron gas which can be created in the inversion layer of a metal-oxidesemiconductor or a semiconductor heterostructure, is exposed to a strong magnetic field, it is observed that the Hall conductance is quantized in units of the elementary conductance $\sigma_{x y}=\nu e^{2} / h$ 257. The initial observation was that $\nu$ is integer 258 but subsequently fractional $\nu$ were discovered, too [259]. In both cases, the quantization is due to the existence of a mobility gap at the Fermi level in the bulk of the sample, although its microscopic origin is different: in the integer effect, disorder leads to localized states while in the fractional effect, correlations condense the particles into a new collective state whose excitations are gapped. Due to the mobility gap in the bulk, transport must take place on the edge of the sample - a 1D manifold.

The basic model for the integer effect was put forward by Halperin [260] and developed further by Büttiker [261] and others [262]. In Fig. 6.5 an annulus with inner radius $r_{1}$ and outer radius $r_{2}$ is considered and the disorder is supposed to be confined to the bulk of the annulus. The edges are shifted upward in energy because of the boundary condition of vanishing wavefunction at the sample boundaries. Although all bulk states at $E_{F}$ are localized (if there are any), at the fields where $\sigma_{x y}$ shows a plateau, low-energy excitations are possible at the edges. The excitations living on the edges are ordinary electrons. They form a 1D chiral Fermi liquid - Fermi liquid now understood in the sense of the higher-dimensional systems. In the Luttinger model (3.1), there is only one of the two dispersion branches: due to the orbital coupling, all electrons move in the same direction, i.e. have a definite chirality. This leaves $g_{4}$ as the only possible interaction. However, due to Zeeman coupling, the electrons are fully spin-polarized, and $g_{4 \perp}$, in principle able to generate charge-spin separation, is quenched. Remains $g_{4 \|}$ which only renormalizes the Fermi velocity and does not destroy the quasi-particle pole in the Green function.

Gapless edge excitations also exist in the fractional quantum Hall effect, and Wen has clarified their nature and dynamics in considerable detail [174]. We attempt to follow his proceeding here, because his way from very general principles (essentially only gauge 
invariance, locality of the theory, and incompressibility of the ground state) to a detailed operator description of the low-energy properties is in some sense opposite to the bulk of our earlier presentation, and highly instructive. Related work has been performed by Stone 263.

We first fix the general form of the action of the edge excitations from the requirement of gauge invariance. Assume that a system displays the quantum Hall effect with $\sigma_{x y}=$ $\nu e^{2} / h$ in an magnetic vector potential $\bar{A}_{\mu}$. We do not know the detailed Hamiltonian, but due to the gap in the quasi-particle excitations, we know that the electrons can be integrated out safely, resulting in an effective Lagrangian

$$
\begin{aligned}
\mathcal{L}_{\mathrm{eff}}\left[\delta A_{\mu}\right] & =\frac{\nu e^{2}}{4 \pi} \delta A_{\mu} \partial_{\lambda} \delta A_{\kappa} \epsilon^{\mu \lambda \kappa}+\frac{1}{4 g_{1}^{2}}\left(\delta F_{01}\right)^{2}-\frac{1}{4 g_{2}^{2}}\left(\delta F_{12}\right)^{2}+\ldots \quad, \\
\delta A_{\mu} & =A_{\mu}-\bar{A}_{\mu} \quad, \quad \delta F_{\mu \lambda}=\partial_{\mu} \delta A_{\lambda}+\partial_{\lambda} \delta A_{\mu}, \quad \mu \quad, \quad \mu=0,1,2 .
\end{aligned}
$$

$\delta F_{\mu \lambda}$ is the strength of the magnetic field. The first term on the right-hand side is called Chern-Simons term, and its coefficient is given by the Hall conductance. The detailed properties of $\mathcal{L}_{\text {eff }}$ are unimportant in what follows. On a compactified space, say a torus, the action $S_{\text {bulk }}=\int d^{3} x \mathcal{L}_{\text {eff }}\left[\delta A_{\mu}\right]$ is invariant under gauge transformations $A_{\mu} \rightarrow A_{\mu}+\partial_{\mu} f(x)$. This is not so on a bounded space where

$$
S_{\text {bulk }}\left[A_{\mu}+\partial_{\mu} f(x)\right]=S_{\text {bulk }}\left[A_{\mu}\right]+\frac{\nu e^{2}}{4 \pi} \int d x_{0} d \sigma f(x) \delta F_{\sigma 0}(x)
$$

The variable $\sigma$ parameterizes the boundary, and $x_{0}=t$. Gauge invariance is violated by a boundary term generated by the Chern-Simons term. A gauge invariant action can now be obtained by adding a boundary action $S_{\text {edge }}$, from which the properties of the boundary excitations can be constructed. $S_{\text {edge }}$ alone must not be gauge invariant and must transform as (6.47) but with a minus-sign in front of the Chern-Simons contribution, and is given by

$$
S_{\text {edge }}=\frac{1}{2} \int d t d \sigma d t^{\prime} d \sigma^{\prime} \delta A_{\alpha}(t, \sigma) R_{(j)}^{\alpha \beta}\left(t-t^{\prime}, \sigma-\sigma^{\prime}\right) \delta A_{\beta}\left(t^{\prime}, \sigma^{\prime}\right), \quad(\alpha, \beta=0, \sigma),
$$

where $R_{(j)}^{\alpha \beta}$ is the time-ordered current-current correlation function (containing, in the notation of Chapter 3, $R_{\rho \rho}, R_{j j}$ and $R_{\rho j}$ ). It has the properties

$$
-k_{\alpha} R_{(j)}^{\alpha \beta}=\frac{\nu e^{2}}{4 \pi} \epsilon^{\alpha \beta} k_{\alpha} \quad, \quad R_{(j)}^{\alpha \beta}\left(k_{\alpha}\right)=R_{(j)}^{\alpha \beta}\left(-k_{\alpha}\right)=\left[R_{(j)}^{\alpha \beta}\left(-k_{\alpha}\right)\right]^{\star}
$$

where $k_{\alpha}=(\omega, k)$ is a reciprocal space vector. We recognize the first equation as a Ward identity, up to the different right-hand side identical to (3.113). The second equation implements the required symmetries (even for the symmetric and odd for the nonsymmetric) and the reality of the correlation functions.

One can imagine knowing the Hamiltonian for the edge excitations and their coupling to the gauge field $A_{\alpha}$. By integrating out the edge excitations, one would then obtain the action (6.48). We proceed inversely and try to get information on the dynamics of the excitations from the effective action. Assume that all edge excitations are gapped, 
and that the theory is local. Then $R_{(j)}$ is smooth near $\omega=0$ and $k=0$. But a smooth function cannot satisfy (6.49). There must thus be gapless excitations (labelled by $i$ ). If their dispersion is linear $\omega(k)=v_{i} k$, and if $R_{(j)}$ has pole structure for the gapless excitations (the gapped ones can only contribute polynomials), its singular part must be of the form

$$
\begin{aligned}
& R_{(j), i j}^{\alpha \beta \text { sing }}(\omega, k)=\frac{\delta_{i j} \eta_{i} S^{\alpha \beta}(\omega, k)}{2 \pi\left(\omega-v_{i} k\right)}, \\
& S^{00}=k \quad, \quad S^{0 \sigma}=S^{\sigma 0}=\left(\omega+v_{i} k\right) / 2, \quad S^{11}=v_{i} \omega .
\end{aligned}
$$

$\eta_{i}=\operatorname{sign}\left(v_{i}\right) q_{i}^{2}$, where $q_{i}$ is the charge of the excitations. There must be an operator $j_{i}^{\alpha}(k)$ which generates a state with energy $\omega(k)=v_{i} k$ from the vacuum. One can then deduce first the vacuum expectation values of the commutators of $j_{i}^{ \pm}(k)=\left[j_{i}^{0}(k) \pm j_{i}^{\sigma}(k) / v_{i}\right] / 2$ from the correlation functions, and then, under some very general further assumptions, the algebra of the operators themselves

$$
\left[j_{i}^{+}(k), j_{j}^{+}\left(k^{\prime}\right)\right]=\left|\eta_{i}\right| k \delta_{i, j} \delta_{k,-k^{\prime}} \quad, \quad\left[H, j_{i}^{+}(k)\right]=c k j_{i}^{+}
$$

For $\operatorname{sign}\left(v_{i}\right) \operatorname{sign}(k)<0$, the operator $j_{i}^{+}(k)$ acts as an annihilation operator. For $\operatorname{sign}\left(v_{i}\right) \operatorname{sign}(k)>$ 0 , it generates the harmonic spectrum of $H$. The operator $j_{i}^{-}(k)$ acts as a null operator.

We recognize the algebra of the $j_{i}^{+}(k)$ (6.51) as a $U(1)$-Kac-Moody algebra. Unlike Eq. (3.169), however, the prefactor of the right-hand side is $\left|\eta_{i}\right|$ rather than unity. This suggests that our edge excitations on each branch $i$ are described by a $U(1)$-Kac-Moody algebra with a central charge $c=\left|\eta_{i}\right|=q_{i}^{2}$. Following our discussion in Section 3.6, we then can construct both an effective action as a bilinear in boson currents $j_{i}^{ \pm}$and in terms of chiral fermions $\Psi_{i}$ coupled to a gauge field, and the latter reads

$$
S_{\text {edge }}=i \sum_{i} \int d t d \sigma \Psi_{i}^{\dagger}\left[\left(\partial_{t}+i q_{i} \delta A_{0}\right)+v_{i}\left(\partial_{\sigma}+i q_{i} \delta A_{\sigma}\right)\right] \Psi_{i}
$$

with charges $q_{i}$ satisfying the sum rule

$$
\sum_{i} \eta_{i}=\sum_{i} \operatorname{sign}\left(v_{i}\right) q_{i}^{2}=\nu e^{2}
$$

For the integer effect ( $\nu$ integer), the charges are integer i.e. the fermions describe real electrons, but for the fractional quantum Hall effect, they are irrational in general. If there is a single branch, we have $q_{i}=\sqrt{\nu} e$. The fermions rather refer to solitons than real electrons. So long as all edge excitations move in the same direction, there is no possibility to open a mass gap. Only when velocities have different signs (an issue we touch upon below) can a gap be opened, as a consequence of backward and Umklapp scattering. In the same way, it should be apparent from the discussion of impurity scattering in Section 4.6 that the edge excitations travelling in the same direction, are not sensitive to scattering by impurities.

For practical calculations, of course, the boson representation is more convenient, and the Hamiltonian corresponding to the boson action is

$$
H=\sum_{i} \sum_{k} \frac{\pi v_{i}}{q_{i}^{2}} \rho_{i}(k) \rho_{i}(-k) \text {. }
$$


There may be also interactions between different excitations, i.e.

$$
H \rightarrow H+\delta H \quad, \quad \delta H=\sum_{i j} \sum_{k} g_{i j} \rho_{i}(k) \rho_{j}(-k)
$$

This has the structure of the Luttinger Hamiltonian (3.1) with $g_{2}$ and $g_{4}$ processes, depending on the sign of the velocities of the branches, or of its multicomponent generalization (6.5). In general, the properties will be different, however, because $c \neq 1$. (6.55) can be diagonalized by a Bogoliubov transformation, leading to new operators $\tilde{\rho}_{i}$, renormalized velocities $\tilde{v}_{i}$, and to renormalized charges $\tilde{q}_{i}=\sum_{j} U_{i j} q_{j}$ where $(U)_{i j}$ is the transformation matrix. It is these renormalized charges and velocities which are experimentally measurable in edge magnetoplasmon excitations. Fractional charges can also be found in the integer effect when several branches of excitations are present. Wen showed that the renormalized fractional charges can be measured experimentally as the strength of the peaks in the absorption spectrum of a rotating electric field. Also the width of the resonances is related through the charges to the edge resistance.

Up to now, we have only considered particle-hole excitations out of the Fermi sea on the edges, i.e. we have restricted to the charge-zero sector of the theory. We now consider the charge excitations on the edges, to give a more systematic basis to the notion of irrational charges, and to construct a relation between the physical fermions $\Psi$ and the bosons living on the edges. We assume a single excitation branch for the moment with $q=\sqrt{\nu}$. There is thus a definite chirality. The fermions act as charge-raising operators

$$
[\Psi, Q]=e \Psi \quad, \quad Q=\int d \sigma j^{0}(\sigma)
$$

The Hilbert space is therefore composed of sectors with charge $Q$, and within these sectors, $j^{+}$creates particle-hole excitations. Within each sector $Q$, it generates the Fock space of the harmonic oscillators defined by the $U(1)$-Kac-Moody algebra (6.51). One can now introduce a bosonic field $\varphi(x)$ via the current and charge

$$
j^{\alpha}(t, \sigma)=\frac{\sqrt{\nu}}{2 \pi} \epsilon^{\alpha \beta} \partial_{\beta} \varphi(t, \sigma) \quad, \quad Q=\sqrt{\nu} N_{\operatorname{sign} v} .
$$

The charge-raising operators of a chiral boson theory are in general, Eq. (3.175)

$$
\Psi(x)=: e^{i \gamma \varphi(x)}:
$$

From the requirement that they anticommute, we derive $\gamma^{2}=2 n+1$, an odd integer. Moreover, $\Psi$ must carry a unit charge. From $[Q, \Psi]$ we find that the charge of $\Psi$ is $\gamma \sqrt{\nu}$ which must equal unity. This imposes a restriction on the filling fractions $\nu$ which can be described by a single branch of excitations $\nu=1 /(2 n+1)$. All other filling fractions must possess more than one edge excitation - a conclusion also verified in numerical calculations [264]. But even in the single-branch situation, a physical electron carrying unit charge, added to the edge, is fragmented into solitonic excitations of charge $\sqrt{\nu}$. However, the charges of excited states are integers, not multiples of $\sqrt{\nu}$.

In principle, one can now calculate all the correlation functions on the edge. Due to the fractional charges, or equivalently to the central charge being different from unity, 
anomalous powers arise even in the one-branch situation, contrary to the standard Luttinger liquid of Chapter 3. This can be seen quite easily from (6.58) where the fractional charge $\nu=K$ plays the same role as the stiffness constant played in Chapter 3 . The mapping is provided by comparing to Eq. (3.67). As an example, the single-particle Green function is

$$
\left\langle\Psi(t, \sigma) \Psi^{\dagger}(0,0)\right\rangle \sim\left(\frac{i}{t+\sigma}\right)^{1 / \nu} .
$$

Rewriting this in the form of a standard Luttinger liquid, one concludes that the chiral single-particle exponent is

$$
\alpha_{\text {chiral }}=\frac{1}{\nu}-1=2 n
$$

for a filling fraction $\nu=1 /(2 n+1)$. The scaling relation between $\alpha$ and $K$ in a chiral Luttinger liquid is different from the non-chiral system. The remarkable fact here is that the exponents become universal and are fully determined by the filling of the Landau levels. This means that one can precisely predict the exponents of the correlation functions probed by specific experiments. Moreover, $\nu$ being a topological invariant, the exponents are expected to be robust against perturbations. Wen has proposed the label "chiral Luttinger liquid" for the low-energy physics of the quantum Hall edge states.

An interesting generalization, which could also be relevant for situations with several edges with opposite velocities, is provided by considering the edge states on a cylinder threaded by the magnetic flux. There are now two edges with excitations moving in opposite directions, and the Luttinger liquid is no longer chiral. Of course, it can be built on the chiral Luttinger liquid of a disk, and the structure of the theory is quite similar to the previous one. There are two important differences, however: (i) charge excitations can be transferred between the edges, which complicates somewhat the quantization rules relating $\gamma$ in $(6.58)$ to the filling fraction $\nu$; (ii) if the edges are not too far from each other, tunneling both of electrons and of Laughlin quasi-particles, the fractionally charged objects introduced earlier, may take place between them. Electron tunneling can be described by the operator

$$
H_{\text {tunnel }}=g \int d x \cos \left[\frac{\varphi(x)}{\sqrt{\nu}}\right],
$$

which is isomorphic in form to our the backscattering operator (4.6). The scaling dimension of this operator is $2-1 / \nu$, and the operator is irrelevant for $\nu<1 / 2$ while it is relevant for $\nu>1 / 2$. This would imply that in the integer effect $(\nu=1)$, a gap could open on the edges when they are brought close enough together. On the other hand, for the fractional effect $\nu=1 /(2 n+1) \leq 1 / 3$, the electron tunneling operator is irrelevant. Tunneling of Laughlin quasi-particles is possible because the two edges are connected by the quantum Hall fluid (and not vacuum), and described by an operator (6.61) where, however, the factor $1 / \sqrt{\nu}$ is replaced by $\sqrt{\nu} 262$. This operator is always relevant. An important problem, however, is that of a local constriction on a Quantum Hall cylinder: here the inter-edge tunneling only takes place at $x=0$, and the dimension of this operator is then $1-\nu$. It is marginal in the integer effect and relevant for all fractional single-edge 
situations. The problem can also be formulated in terms of scattering off impurities in a non-chiral Luttinger liquid [169, and out conclusion agrees with the analysis of Section 4.6 .

The dynamical excitations on the edges have a very rich structure which reflects the topological order in the quantum Hall effect. Wen [174, 262] and others [263] have developed here a chiral Luttinger liquid theory as a framework for a detailed description of their properties. It is similar to the Luttinger liquid discussed in the preceding chapters but differs (at least) in one important way: the charges of the edge excitations are fractional (or irrational), and the universality classes of the chiral and standard Luttinger liquids are different: the central charge describing the edge excitations is different from unity. 


\section{Chapter 7}

\section{The normal state of quasi-one-dimensional metals - a Luttinger liquid?}

A wide variety of materials with low-dimensional structural and electronic properties is available now, and experimentalists have used them to search for Luttinger liquid correlations. In the main body of this chapter, we shall concentrate on the quasi-1D organic conductors such as TTF - TCNQ and superconductors like $(T M T S F)_{2} X$ or the related (nonsuperconducting) series $(T M T T F)_{2} X$. We summarize evidence that electronic correlations are important in these materials, that in their normal state, they are sufficiently anisotropic so that 1D models of interacting electrons are indeed relevant for their description, and that experiments can be interpreted consistently within the theoretical framework set up in this article. At the end, we briefly touch upon semiconductor heterostructures, a new and rapidly growing branch in the field of correlated 1D electrons.

\subsection{Organic conductors and superconductors}

Tetrathiafulvalene-tetracyanoquinodimethane $(T T F-T C N Q)$ crystalizes in a herringbone pattern of two segregated stacks of TTF and TCNQ molecules, respectively. A charge transfer from the TTF to the TCNQ molecules of 0.57 electron/molecule produces partially filled electron- and hole-like bands, i.e. the material behaves as a two-chain conductor.

Between $54 \mathrm{~K}$ and $38 \mathrm{~K}, T T F-T C N Q$ undergoes a series of phase transitions into a CDW state, accompanied by a periodic lattice modulation due to electron-phonon coupling. Although the traditional picture due to Peierls would consider electron-phonon interaction as the driving force of such a CDW transition [5, 265], the actual situation is more complicated, probably radically different.

In fact, there is ample evidence for important repulsive interactions on both chains. In $\mathrm{X}$-ray experiments, diffuse X-ray scattering is not only detected at $2 k_{F}$ but surprisingly also at $4 k_{F}$, even up to very high temperature [266, 267]. While $2 k_{F}$-fluctuations are 
expected as precursors of the Peierls transition [265], the existence of $4 k_{F}$ fluctuations can only be explained assuming sizable Coulomb correlations [58, as can be seen from comparing the exponents of the $2 k_{F^{-}}$and $4 k_{F^{-}} \mathrm{CDW}$ correlation functions (3.92) and (3.93). Notice from Eqs. (4.65) that coupling to lattice phonons $\left(Y_{2}^{(p h)}=0\right)$ reduces $K_{\rho}$. When electronic correlations are dominant, phonons can enhance them further, and they certainly outweigh the logarithmic "advantage" of the SDWs against the $2 k_{F}$-CDWs. The Pauli susceptibility is significantly enhanced over the free electron value [268, 269] and the finite frequency optical conductivity is much larger than the dc-conductivity 167. Further evidence for Coulomb correlations stems from the analysis of systematic variations of physical properties through entire families [270] of closely related compounds such as $(N M P)_{x}(\text { Phen })_{1-x}(T C N Q)$ 110, 271] or the $1: 2-T C N Q$ salts 272].

There is thus an alternative, well supported view that the CDWs in TTF - TCNQ are, in fact the consequence of electronic correlations rather than of electron-phonon interaction; the latter then would just probe the electronic fluctuations without feeding back on them in any significant manner, and couple the preformed charge density modulation into the lattice and make it visible to X-rays. This is different from other CDW systems to be discussed in Section 7.2 below.

Closely related are the single-chain conductors $(T M T S F)_{2} X$ and $(T M T T F)_{2} X$ ("Bechgaard salts"), where TMTSF stands for the molecule tetramethyl-tetraselenafulvalene. A sketch of this molecule and of the stacking pattern, immediately suggestive of onedimensionality, is shown in Figure 7.1. (TMTSF $)_{2} P F_{6}$ undergoes a metal-insulator transition into a SDW state at ambient pressure at $T_{S D W}=12 K$. Evidence for SDWs is provided by peculiar NMR relaxation behaviour [15] but most convincingly by observation of the nonlinear conductivity associated with a sliding SDW [273]. Under 12 kbar pressure, however, superconductivity can be stabilized at $T_{c}=0.9 \mathrm{~K}$ [274]. Other members of the family show superconductivity, too [275]. By substituting the four selenium atoms by sulfur, one obtains $(T M T T F)_{2} X$. Generically, these sulfur-based systems undergo a charge localization transition ("Wigner crystallization") around $200 K$ (seen e.g. as a minimum in the resistivity) and reduce to an effective spin chain below. Finally, around $20 \mathrm{~K}$, one observes spin-Peierls transitions into a spin-singlet state, accompanied by a lattice deformation [23]. However, by applying pressure, a behaviour more akin to the SDW state of $(T M T S F)_{2} P F_{6}$ can be obtained.

The importance of Coulomb interactions in the $(T M T S F)_{2} X$ and $(T M T T F)_{2} X$ is more readily apparent than in $T T F-T C N Q$. We discussed various Luttinger liquid correlation functions in Chapter 4 , and SDW correlations require repulsive interactions. In the $(T M T T F)_{2} X$-series, the Coulomb repulsion is even stronger than in $(T M T S F)_{2} X$ : the charge localization transition around $200 \mathrm{~K}$ can be interpreted as a transition into a $4 k_{F}$ CDW [58, 89, 110]. Other properties indicate strong Coulomb interaction, too. The Pauli susceptibility of the conduction electrons is enhanced considerably $(\times 3-5)$ with respect to a simple band picture [276]. Moreover, it is temperature dependent and, at low temperature, close to what is expected for a 1D antiferromagnet [23]. Optical properties are also unconventional. Apparently it is observed that $\sigma(\omega)$ in the infrared is higher than the dc-conductivity which is not compatible with free electrons and suggests rather 
localized charges. In these infrared measurements one also observes totally symmetric molecular vibrations [277, 278]. These vibrations are IR-forbidden for free electrons but can be activated by local charge modulations such as a CDW [279]. In the $(T M T S F){ }_{2} X$ and $(T M T T F)_{2} X$ such a CDW would be at $4 k_{F}$ and the observation of the activated vibrations in the IR then suggests a considerable degree of charge localization. Strong electronic repulsion generates antiferromagnetic spin fluctuations which can be, and have been, observed in NMR [280, 281]. There is also a detailed theory for the analysis of these experiments which lends further support to a strongly correlated picture for the electrons in the Bechgaard salts [282, 283].

The basic building blocks of the materials discussed in the preceding section are large planar molecules with $\pi$-orbitals directed out of the molecular plane. In general, the lattice parameters, the lattice dynamics, and the elastic constants are anisotropic though not strongly so, and are best viewed as three-dimensional [15]. The electronic properties, however, are strongly anisotropic. Essentially, the overlap between wave functions on neighbouring molecules, and matrix elements of a Hamiltonian between them, both depend exponentially on the intermolecular separation: minor variations in the structure are then dramatically amplified in the electron dynamics. The sensitive dependence on the intermolecular distances is further amplified by the strong directionality of many of the molecular orbitals involved. However, relatively short distances between $S e$ or $S$ atoms in the $(T M T S F)_{2} X$ and $(T M T T F)_{2} X$ give nonnegligible interstack contacts and some effectively $2 \mathrm{D}$ character to the $(T M T S F)_{2} X$ salts [284].

The central question is therefore if the Fermi surface of these organic conductors is closer to the parallel sheets characterizing 1D or to the cylinder obtained for layered $2 \mathrm{D}$ materials, i.e. open vs. closed orbits, and if, for given external parameters $(T, P)$, the electrons hop coherently or not from one chain to its neighbour. There seems to be general agreement [23] that the picture of two sheets, warped by the finite hopping integrals perpendicular to the chains, is most appropriate. Values often used are $t_{\|} \equiv t_{a}=$ $150 \ldots 200 \mathrm{meV}, t_{\perp} \equiv t_{b}=20 \ldots 30 \mathrm{meV}$, and $t_{c}<t_{b} / 10$ for $(T M T S F)_{2} X$ [23 although next-nearest-neighbour transfer integrals also seem to play some role [284]. Apparently $T T F-T C N Q$ is significantly more anisotropic and the Fermi surface has been suggested to consist out of two parallel, diffuse sheets. Representative transfer integrals are $t_{\|}=$ $110 \mathrm{meV}$ for the TCNQ chain, $t_{\|}=50 \mathrm{meV}$ for TTF [15], and $t_{\perp}$ as low as $5 \mathrm{meV}$ [285.

Transport measurements probe the anisotropy and coherence of the electron dynamics. In optical absorption, a pronounced plasma edge in the $1 \mathrm{eV}$-range is observed for electric fields polarized parallel to the chain axis both in TTF - TCNQ and $(T M T S F){ }_{2} X$, indicating band formation along the chains, and longitudinal bandwidths of the order of $1 \mathrm{eV}$ have been derived within simple tight-binding models. In $(T M T S F)_{2} P F_{6}$, a transverse plasma edge at about $0.1 \mathrm{eV}$, implying coherent perpendicular transport, is observed only at $T=25 K$ 15. In TTF - TCNQ, to the best of the author's knowledge, the establishment of a transverse plasma edge has never been observed. These measurements support the picture of a very anisotropic band and rule out the possibility of a closed Fermi surface in these materials.

Depending on the magnitude of $\tau_{\|} t_{\perp} / \hbar$, the transverse transport can be either coherent 
or diffusive. Here $\tau_{\|}$is the on-chain collision time after which the electron wave function looses its coherence, i.e. a measure of cleanliness, and $t_{\perp}<t_{\|}$is assumed. The transverse escape rate $1 / \tau_{\perp} \approx 2 \pi \tau_{\|} t_{\perp}^{2} / \hbar$ can be measured by NMR [286]. The long-wavelength spin fluctuations on the chains are certainly diffusive and then described by a $1 \mathrm{D}$ random walk which gives a $1 / \sqrt{\omega_{e}}$ power spectrum to the NMR relaxation rate $1 / T_{1} T$ and strong fielddependent deviations from the Korringa law through the electronic Larmor frequency $\omega_{e}$. At low-fields, the spin dynamics becomes $3 \mathrm{D}$ and $1 / T_{1} T$ is field independent. A crossover is observed for $\omega_{e} \tau_{\perp} \approx 1$ in TTF - TCNQ [286] and $(T M T S F)_{2} X$ [287]. $\tau_{\|}$ can be estimated e.g. from conductivity. Putting things together, one concludes that, in $T T F-T C N Q$, the perpendicular transport is always diffusive while in $(T M T S F)_{2} P F_{6}$, it is diffusive at higher temperature but transverse coherence is established at lower temperatures. One also obtains the above values of $t_{\perp}$. This suggests that theories discussing the establishment of transverse coherence in the single- and two-particle dynamics can be critically tested here.

The information available points towards a picture of strong Coulomb interaction and pronounced one-dimensionality. What is the experimental evidence in favour of Luttinger liquid behaviour in these "metals"? The instabilities observed at low temperatures are certainly suggestive of $1 \mathrm{D}$ physics. Of course, they do not tell us much about the normal-state properties; still the observation of high-temperature $4 k_{F^{-}} \mathrm{CDW}$ fluctuations in $T T F-T C N Q$ [266, 267] places constraints on the effective Luttinger parameters: $K_{\rho}<1 / 2$ for their divergence, and $K_{\rho}<1 / 3$ for them being stronger than the $2 k_{F^{-}}$CDWs. These low values of $K_{\rho}$ indicate that a simple Hubbard model is certainly inappropriate for the description of this compound, and that longer range interactions cannot be neglected. The optical spectrum of $T T F-T C N Q$ is very unusual (Figure 7.2). There is a pronounced pseudogap present already at $85 \mathrm{~K}$, i.e. far above the Peierls temperature, which deepens as the temperature is lowered into the CDW phase. It is tempting to connect this with the pseudogap observed in the density of states [167, and in fact, $\sigma(\omega)$ is not incompatible with the expression (4.75) derived by Ogata and Anderson [166], if the large $\alpha$-values implied by the $4 k_{F}$-fluctuations are inserted.

In the $(T M T S F){ }_{2} X$-materials, NMR has uncovered anomalous correlations [280, 281] (Figure 7.3). The spin-lattice relaxation rate $T_{1}^{-1}$ strongly deviates from the Korringa law $T_{1}^{-1} \propto T$ at lower temperatures. This is believed to result from the temperature dependent SDW-correlations. In fact, $T_{1}^{-1}$ contains two contributions, from the long-

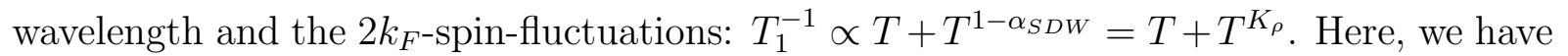
a quantity which can give direct information on $K_{\rho}$ ! Below a certain temperature $T_{0}$, the SDW contribution $T^{K_{\rho}}$ dominates over the long-wavelength part $T$. From the latest work [281, one deduces $K_{\rho} \sim 0.15$. This is a surprisingly big value, and again indicates effective strong and long-range interactions. One problem here, in contrast to TTF - TCNQ is that the $4 k_{F}$-fluctuations predicted in this limit, are not observed in X-rays (but they are neither in the $(T M T T F)_{2} X$-series where $4 k_{F}$-localization is established quite firmly).

Similar values of $K_{\rho}$ are suggested by the photoemission experiments shown in Figure 7.4. This experiment measures the occupied $(\omega<0)$ part of the single-particle density of states $N(\omega)$ (3.87). There is no spectral weight at the Fermi surface, and it rises smoothly 
as one goes to lower energies. The origin of the peak at $-1 \mathrm{eV}$ is not clear - this scale lies at the lower edge of the valence band, or even below. Close to the Fermi energy, the smooth variation is compatible with the Luttinger liquid form $|\omega|^{\alpha}$ if one assumes an exponent $\alpha>1$. The NMR- $K_{\rho}=0.15$ implies $\alpha \sim 1.25$, and this value describes the data quite well.

Summarizing, there are experimental indications in favour of Luttinger liquid correlations in these quasi-1D organic conductors which, if taken serious, would place them in the limit of strong, long-range effective interactions. The experiments described probe, however, only the anomalous operator-dimensions. There has been no successful attempt to measure charge-spin separation. From Section 3.4, this would require angle-resolved photoemission or inelastic neutron scattering. However, the relevance of a Luttinger liquid description, even within a 1D framework, has been questioned 2289. In fact, the $(T M T S F)_{2} X$-materials are slightly dimerized, and therefore the Fermi level lies in an effectively half-filled subband, where even weak interactions can lead to charge localization and an associated charge gap, cf. Chapter 5. The unusual phenomena observed would, in this view, either be extrinsic or mainly reflect the consequences of a Mott transition. At the time of writing, the controversy over the appropriate model for describing the organic conductors is pretty open.

\subsection{Inorganic charge density wave materials}

Many inorganic crystals, such as e. g. $\mathrm{K}_{0.3} \mathrm{MoO}_{3},\left(\mathrm{TaSe}_{4}\right)_{2} \mathrm{I}, \mathrm{NbSe} e_{3}$ etc. undergo, at temperatures between $50 K$ and $250 K$, a Peierls transition into a CDW ground state, giving rise to fascinating nonlinear transport phenomena 290. Apart $\mathrm{NbSe}_{3}$ which apparently is quite $3 \mathrm{D}$ and where pieces of a Fermi surface persist even into the CDW state, the materials, generically, are rather 1D and do not show indications of strong electronic correlations.

The driving mechanism for CDW formation is the electron-phonon interaction [5], and accordingly, this interaction is supposed to be the dominant one in these materials. Considerable success in describing their normal state properties has been achieved based

on the model of a fluctuating Peierls insulator [291]. The basic idea here is that 1D fluctuations will lower the actually observed critical temperature $T_{P}$ below the $1 \mathrm{D}$ meanfield temperature $T_{P}^{M F}$ by as much as a factor of $4-5$. In the fluctuation regime, there is a pseudo-gap in the electronic density of states which develops into a real gap only at $T_{P}$ and which accounts for the unusual thermodynamic and transport properties above $T_{P}$. There is no pseudo-gap left beyond $T_{P}^{M F}$.

The fluctuating Peierls insulator model is a single-particle picture, i.e. charge and spin degrees of freedom behave symmetrically. This is observed in some materials, such as $(\mathrm{TaSe} 4)_{2} \mathrm{I}$, but not in others, e.g. $\mathrm{K}_{0.3} \mathrm{MoO}_{3}$, where the dc-conductivity is totally unaffected by the conjectured pseudo-gap while the susceptibility shows temperature dependence reminiscent of thermal activation. Moreover, the transition in conductivity is extremely sharp - but gradual in susceptibility [292]. The Lee-Rice-Anderson picture 
seems to imply that above $T_{P}^{M F}$ the system reduces to a normal metal. The corresponding temperature-independent Pauli susceptibility above $T_{P}^{M F}$ has, however, not been observed in any of the 1D CDW materials.

Photoemission experiments question the validity of the picture of a fluctuating Peierls insulator. As in the organic conductors, the single-particle density of states shows no significant weight at the Fermi energy in the blue bronze $K_{0.3} \mathrm{MoO}_{3}$, and appreciable spectral weight is only found a sizable fraction of an $\mathrm{eV}$ below 293. Angle-resolved studies on related materials either show a fading away of the signal as the Fermi surface is approached [294, or weight dispersing some finite energy below $E_{F}$ 295. Clearly, there have been speculations about 1D correlations at the origin of the mysterious behaviour.

Experimental evidence points against simple Luttinger liquid behaviour in these CDW materials. On the other hand, our renormalization group analysis (4.65) in Section 4.5 shows that, in such a $1 \mathrm{D}$ scenario, a spin gap $\Delta_{\sigma}$ must open as a precursor of CDW formation while the charge fluctuations remain massless in strictly 1D. Such a system is in the Luther-Emery universality class [186]. The Pauli susceptibility rising with increasing temperature, found in all CDW materials, can be interpreted as evidence for such a spin gap. The conductivity of the blue bronze $K_{0.3} \mathrm{MoO}_{3}$ also provides evidence for massless charge fluctuations [292]. One can compute, at least the diagonal part of the single-particle density of states for this model and finds 53

$$
N(\omega) \approx \Theta\left(|\omega|-\Delta_{\sigma}\right)\left(|\omega|-\Delta_{\sigma}\right)^{2 \gamma_{\rho}}
$$

where $\gamma_{\rho}$ has been defined in (3.84). Such a behaviour is consistent with the photoemission properties of the blue bronzes. We note, however, that (i) optical experiments [296] are in excellent agreement with the fluctuating-Peierls-insulator model when one goes beyond the Lee-Rice-Anderson treatment and includes the thermally excited motion of the lattice [297, and their precise relation to the photoemission experiments is not understood to date; (ii) there is no evidence for charge-spin separation in CDW-materials other than $K_{0.3} \mathrm{MoO}_{3}$ which therefore are not in the Luther-Emery universality class.

Similar unusual photoemission behaviour: absence of spectral weight at the Fermi surface and a smooth rise below, has also been reported for another inorganic 1D material, $\mathrm{BaVS}_{3}[298]$.

\subsection{Semiconductor heterostructures}

Semiconductor heterostructures may open a wide new field for the study of 1D interacting electrons, in regimes usually inaccessible to organic crystals. There are two principal directions: (i) quantum wires and (ii) quantum Hall effect edge states.

Traditionally, a 2D electron gas is induced at interfaces in such structures by applying a gate voltage across the structure. Very recent progress has allowed the fabrication of narrow channels in the heterostructures where the electrons can be confined [299] into a quantum wire. The electronic system can be made truly one-dimensional by appropriate design of the structure. Periodic conductance oscillations are observed as a function of the 
carrier density, and it has been speculated that they could be either due to the formation of a charge density wave or of a Wigner crystal [299]. Wigner crystal formation, i.e. the formation of $4 k_{F}$-CDWs has been discussed in Chapter 1 [89, 90, 112, 148]. Furthermore, the 1D channel can be constricted, and we can verify the predictions for transport through an impurity, discussed in Section 4.6 .

Such a constriction can also be built on samples showing the quantum Hall effect. From Section 6.3, we know that the edge states are described as chiral Luttinger liquids. For the $\nu=1 / 3$ quantum Hall state where we have a single edge, the Luttinger stiffness constant is $K=\nu=1 / 3$. The exponents of all correlation functions are fully determined by the filling-factor $\nu$ of the Landau levels! Eq. (4.82) shows that the corrections to the Luttinger liquid conductance diverge as $T \rightarrow 0$. An equation equivalent for the opposite case, tunneling across the constriction, predicts the tunneling conductance across the constriction to vary as $G(T) \sim T^{4}$ [169]. Such an experiment has been performed 300, and the result shown in Figure 7.5 is in accurate agreement with the theoretical prediction. For comparison, a temperature independent conductance is expected for a Fermi liquid $(K=1)$, and is indeed found in the integer $\nu=1$-state 300. The scaling with applied point-contact voltage is similar. Again, for the $\nu=1 / 3$-state, a strong variation with voltage is found while the variation for $\nu=1$ is much weaker [300]. It would be very interesting to see if such constrictions can also be used to separate charge and spin of the electrons on the two sides, as suggested in Section 4.6. 


\section{Chapter 8}

\section{Summary}

We have gone a long way from the simplest 1D model Hamiltonian, the Luttinger model, to exotic correlations in complicated materials, often uncovered only under extreme conditions. It is certainly useful to briefly summarize the essential steps and achievements.

Fermi liquid theory based on a quasi-particle picture as in higher dimensions, does not work in 1D because of two new features with respect to 3D: a logarithmic divergence in the particle-hole bubble, due to the perfect nesting of the 1D Fermi surface, and because of charge-spin separation in 1D. Both effects are connected to the fact that momentum transfer cannot be neglected in the scattering processes in 1D.

Quasi-particles are unstable in 1D, and the elementary excitations are bosonic collective charge and spin fluctuations. The Luttinger model incorporates these essential features and can therefore be taken as a basis for the description of gapless 1D quantum systems. This model has been solved by several methods: bosonization, equation of motion and Green's function techniques, conformal field theory. All of them are related to the symmetries and conservation laws of the 1D Fermi surface, but incorporate them in a different manner. While the use of Ward identities in the Green's function method emphasizes strong similarities to the Fermi liquid, bosonization rather displays the differences. In particular, we used bosonization to compute correlation functions. They decay as nonuniversal power-laws, and the scaling relations between their exponents are parameterized by a single effective coupling constant $K_{\nu}$ per degree of freedom. In addition, there is a renormalized velocity of each collective mode. It renormalizes the thermodynamic and transport properties, but its most spectacular consequence, charge-spin separation, is only visible in dynamical correlations at large wave-vector, such as the single-particle spectral function close to $k_{F}$ or the charge and spin structure factors at $2 k_{F}$.

The effective coupling constant is defined from the velocities associated with three different low-energy excitations: particle-hole excitations, charge $\left( \pm k_{F}\right.$-symmetric addition of particles) and current ( $k_{F} \leftrightarrow-k_{F}$-transfer of particle) excitations, and thus from the eigenvalue spectrum alone. This structure persists in a low-energy subspace of more complicated models containing nonlinear dispersion, (irrelevant) large-momentum transfer scattering, coupling to external degrees of freedom, etc., and carries to the notion of a "Luttinger liquid". It implies that the low-energy properties of these models are described by a renormalized Luttinger model, provided their excitations are gapless. The 
Luttinger liquid is the universality class of these gapless 1D quantum systems. By a controlled mapping of 1D models ranging from the Hubbard model to electron-phonon systems, an asymptotically exact solution of the 1D many-body problem is achieved. We have discussed several procedures, some applicable only where an exact solution by Bethe Ansatz is possible, others generally applicable and therefore also suited for non-soluble models. We often used renormalization group which is not as powerful as methods based on an exact eigenvalue spectrum because it is based on perturbative developments and thus limited to weak coupling. While failing quantitatively at stronger coupling, most of its predictions are qualitatively valid beyond the weak-coupling range. In particular, it allows to derive logarithmic corrections to correlation functions which lift the unphysical degeneracies implied by their exponents. Moreover, it is flexible enough to allow a treatment of problems beyond the reach of both exact and numerical solutions such as electron-phonon coupling and scattering off impurities. It is therefore essential for a determination of transport properties, to which we devoted much space. Of course, Luttinger liquid behaviour is also found in multi-band and multi-component models, and most methods generalize straightforwardly to these problems.

Not all 1D fermion systems are Luttinger liquids. When backward or Umklapp scattering operators become relevant, as they do for attractive interactions or commensurate band-fillings, respectively, a gap opens in the spin or in the charge channel. Passing back and forth between strong- and effective weak-coupling models, a detailed picture of their properties can be constructed. We have done this in particular for the Mott transition in commensurate, repulsively interacting systems, and emphasized phase diagrams, critical interaction strengths, and the scaling behaviour of transport properties and correlations.

The solution of the Luttinger, Hubbard and other models relies in an essential way on the strong conservation laws provided by the small phase space of 1D. An important problem therefore is the stability of the Luttinger liquid with respect to transverse coupling. Coupling by interchain Coulomb interaction often gives only quantitative modifications of the 1D behaviour, except for backscattering which can stabilize charge density wave correlations into a long-range ordered phase. Interchain tunneling on the other hand can lead to transverse coherence either in the single- or in the two-particle dynamics, depending on the on-chain correlations, and stabilize a variety of phases. If a single-particle crossover occurs first, as the temperature is lowered, the Peierls-Cooper interference in destroyed, and a low-temperature phase transition may take place in one channel alone. Despite intense research, the normal-state properties above such a transition, are not fully clear to date. There seems to be agreement that charge-spin separation is an essential feature in this situation, but there is disagreement on the extent to which it confines the electron dynamics onto a single chain. A two-particle crossover may occur before the single-particle one, and the on-chain correlations are then propagated by transverse particle-particle or particle-hole pair hopping, leading again to low-temperature phase transitions. Here, the normal state is of 1D Luttinger type. An important problem is the Hubbard model on two or more coupled chains, and at least the two-chain variant provides evidence for possible superconducting correlations at repulsive interactions.

Many experiments have provided evidence for Luttinger liquid correlations in low- 
dimensional materials, although there is often some controversy about their precise interpretation. Quasi-1D organic conductors and superconductors, for example, show (TTF$T C N Q$ ) diffuse X-ray scattering at $2 k_{F}$ and $4 k_{F}$ and strongly depressed low-frequency optical conductivity, and others $\left((T M T S F)_{2} X\right)$ power-law deviations from the Korringa law in NMR, and vanishing spectral weight at the Fermi surface in photoemission. These experiments point towards really strong, and in particular long-range, interactions. Instrumental to this conclusion are bounds on the Luttinger coupling constants $K_{\nu}$ derived from mapping various lattice models onto the Luttinger model. Suggesting single-particle exponents $\alpha$ in excess of unity, in fact, there are no known lattice models which naturally would provide values so big. This certainly is a major problem for future research and for the modelling of these materials.

Similarly surprising is the absence of spectral weight at $E_{F}$ in inorganic charge density wave systems although these are believed to be dominated by electron-phonon coupling. From our discussion of phonon-coupled Luttinger liquids, we have suggested that they fall into the Luther-Emery universality class, and that spin gap formation is quite generally a precursor of charge density wave formation. Studies of spectral functions for these models are certainly called for.

Finally, edge state transport in the fractional quantum Hall effect is an exciting new area of low-dimensional physics. We have discussed how these gapless edges can be modelled as chiral Luttinger liquids. While they are similar to those discussed before, having a central charge $c \neq 1$, they fall into a different universality class. They also have power-law correlations, but their charges in general are irrational. Still, much of what has been said about correlation functions and transport for the normal Luttinger liquid carries over to the chiral variant. The remarkable feature here is that, at least for the single-edge situations, the renormalized coupling constant $K$ is fully determined by the Landau level filling fraction $K=\nu$, and therefore all correlation exponents (i) are known in advance, and (ii) can be tuned accurately by varying $\nu$. A recent experiment on tunneling through a barrier on the edges at $\nu=1 / 3$ is in agreement with the chiral Luttinger prediction and apparently provides a first evidence for the relevance of this picture.

\section{Acknowledgements}

I should like to thank the following colleagues for stimulating interaction, helpful suggestions, criticism, and essential support, often over many years: Jim Allen, Natan Andrei, Claude Bourbonnais, Sergei Brazovskii, Helmut Büttner, David Campbell, Laurent Caron, Michele Fabrizio, Florian Gebhard (especially for many constructive comments on the present article), Thierry Giamarchi, Daniel Malterre, Thierry Martin, Eugene Mele, Philippe Nozières, Jürgen Parisi, Jean-Paul Pouget, Dierk Rainer, Mario Rasetti and the Institute for Scientific Interchange in Torino, Heinz Schulz, Markus Schwoerer, AndréMarie Tremblay, Joe Wheatley. The responsibility for flaws in this article is, however, entirely mine. My research is supported by Deutsche Forschungsgemeinschaft through SFB 279-B4. 


\section{Bibliography}

[1] L. D. Landau, Sov. Phys. JETP 3, 920 (1957); 5, 101 (1957); 8, 70 (1959).

[2] P. Nozières, Interacting Fermi Systems, W. A. Benjamin Inc, New York (1964).

[3] F. D. M. Haldane, J. Phys. C 14, 2585 (1981).

[4] J. M. Luttinger, Phys. Rev. 119, 1153 (1960).

[5] R. Peierls, Quantum Theory of Solids, Oxford University Press, London (1955).

[6] J. Bardeen, L. N. Copper, and J. R. Schrieffer, Phys. Rev. 108, 1175 (1957).

[7] J. M. Luttinger, J. Math. Phys. 4, 1154 (1963).

[8] S. Tomonaga, Prog. Theor. Phys. 5, 544 (1950).

[9] D. C. Mattis and E. H. Lieb, J. Math. Phys. 6, 304 (1963).

[10] K. B. Efetov and A. I. Larkin, Sov. Phys. JETP 42, 390 (1976).

[11] P. W. Anderson and Y. R. Ren, Proceedings of the Los Alamos Conference on High$T_{c}$-Superconductivity, Addison Wesley Publ. Comp., 1990, p. 3; P. W. Anderson, Phys. Rev. Lett. 64, 1839 and 65, 2306 (1990).

[12] J. R. Engelbrecht and M. Randeria, Phys. Rev. Lett. 65, 1032 (1990); D. Coffey and K. S. Bedell, ibid. 71, 1043 (1993).

[13] C. M. Varma, P. B. Littlewood, S. Schmitt-Rink, E. Abrahams, and A. E. Ruckenstein, Phys. Rev. Lett. 63, 1996 (1989); N. Mitani and S. Kurihara, Physica C 192, 230 (1992).

[14] I. Perakis, C. M. Varma, and A. E. Ruckenstein, Phys. Rev. Lett. 70, 3467 (1993); G.-M. Zhang and L. Yu, ibid. 72, 2474 (1994); C. Sire, C. M. Varma, A. E. Ruckenstein, and T. Giamarchi, ibid. p. 2478. See also G. M. Eliashberg, JETP Lett. 46, S81 (1988); B. R. Alascio and C. R. Proetto, Sol. St. Comm. 75, 217 (1990).

[15] D. Jérôme and H. J. Schulz, Adv. Phys. 31, 299 (1982).

[16] Proceedings of recent conferences on Synthetic Metals, e. g. Synth. Met. 27 (1988) - 29 (1989); $4 \mathbf{1}-\mathbf{4 3}$ (1991); $\mathbf{5 5}$ - $\mathbf{5 7}$ (1993); $6 \mathbf{9}$ - $\mathbf{7 1}$ (1995). 
[17] P. Nozières and A. Blandin, J. Phys. (Paris) 41, 193 (1980); N. Andrei and C. Destri, Phys. Rev. Lett. 52, 364 (1984); A. W. W. Ludwig and I. Affleck, Phys. Rev. Lett. 67, 3160 (1991).

[18] J. Sólyom, Adv. Phys. 28, 201 (1979).

[19] V. J. Emery, in Highly Conducting One-Dimensional Solids, ed. by J. T. Devreese, R. E. Evrard, and V. E. van Doren, Plenum Press, New York (1979).

[20] I. Affleck, in: Fields, Strings, and Critical Phenomena, ed. by E. Brézin and J. Zinn-Justin, Elsevier Science Publishers B. V., Amsterdam, 1989.

[21] Yu. A. Firsov, V. N. Prigodin, and Chr. Seidel, Phys. Rep. 126, 245 (1985).

[22] C. Bourbonnais and L. G. Caron, Int. J. Mod. Phys. B 5, 1033 (1991).

[23] D. Jérôme, Science 252, 1509 (1991).

[24] J. M. Williams, A. J. Schultz, U. Geiser, K. D. Carlson, A. M. Kini, H. H. Wang, W.-K. Kwok, M.-H. Whangbo, and J. E. Schirber, Science 252, 1501 (1991).

[25] Low-Dimensional Conductors and Superconductors, ed. by D. Jérôme and L. G. Caron, Plenum Press, New York, 1987.

[26] B. Sutherland, in Exactly Solvable Problems in Condensed Matter and Relativistic Field Theory, Lecture Notes in Physics 242, 1 (1985).

[27] V. E. Korepin, N. M. Bogoliubov, and A. G. Izergin, Quantum Inverse Scattering Method and Correlation Functions, Cambridge University Press, Cambridge, 1993.

[28] Yu. A. Izyumov and Yu. N. Skryabin, Statistical Mechanics of Magnetically Ordered Systems, Consultants Bureau, New York, 1988, chapter 5.

[29] A. A. Belavin, A. M. Polyakov, and A. B. Zamolodchikov, Nucl. Phys. B 241, 333 (1984); Fields, Strings, and Critical Phenomena, ed. by E. Brézin and J. ZinnJustin, Elsevier Science Publishers B. V., Amsterdam, 1989; C. Itzykson and J.-M. Drouffe, Statistical Field Theory, Cambridge Universtity Press, Cambridge, 1989, vol. 2; Y. Grandati, Ann. Phys. Fr. 17, 159 (1992); A. W. W. Ludwig, Trieste Lectures 1992.

[30] Yu. A. Bychkov, L. P. Gorkov, and I. E. Dzyaloshinskii, Sov. Phys. JETP 23, 489 (1966).

[31] R. Heidenreich, R. Seiler, and A. Uhlenbrock, J. Stat. Phys. 22, 27 (1980).

[32] A. Luther and I. Peschel, Phys. Rev. B 9, 2911 (1974).

[33] D. C. Mattis, J. Math. Phys. 15, 609 (1974). 
[34] I. E. Dzyaloshinskii and A. I. Larkin, Sov. Phys. JETP 38, 202 (1974).

[35] H. U. Everts and H. Schulz, Sol. State Comm. 15, 1413 (1974).

[36] M. Apostol, J. Phys. C 16, 5937 (1983).

[37] This argument was suggested by Michele Fabrizio.

[38] R. de L. Kronig, Physica 2, 968 (1935).

[39] E. Witten, Commun. Math. Phys. 92, 455 (1984).

[40] S. Mandelstam, Phys. Rev. D 11, 3026 (1975).

[41] F. D. M. Haldane, Phys. Rev. Lett. 47, 1840 (1981).

[42] H. J. Schulz, Int. J. Mod. Phys. B 5, 57 (1991).

[43] W. Kohn, Phys. Rev. 133, A171 (1964); X. Zotos, P. Prelovsek, and I. Sega, Phys. Rev. B 42, 8445 (1990); B. S. Shastry and B. Sutherland, Phys. Rev. Lett. 65, 243 (1990).

[44] T. Giamarchi, Phys. Rev. B 44, 2905 (1991).

[45] T. Giamarchi and A. J. Millis, Phys. Rev. B 46, 9325 (1992).

[46] W. Metzner and C. Di Castro, Phys. Rev. B 47, 16107 (1993).

[47] R. Shankar, Int. J. Mod. Phys. B 4, 2371 (1990).

[48] H. J. Schulz, Phys. Rev. Lett. 64, 2831 (1990).

[49] F. D. M. Haldane, Phys. Rev. Lett. 45, 1358 (1980).

[50] J. Voit, Phys. Rev. B 45, 4027 (1992).

[51] Y. Suzumura, Prog. Theor. Phys. 63, 5 (1980).

[52] H. J. Schulz, J. Phys. C 16, 6769 (1983).

[53] J. Voit, J. Phys. CM 5, 8305 (1993).

[54] K. Schönhammer and V. Meden, Phys. Rev. B 47, 16205 (1993) and (E) 48, 11521 (1993).

[55] A. Theumann, J. Math. Phys. 8, 2460 (1967).

[56] H. Gutfreund and M. Schick, Phys. Rev. 168, 418 (1968).

[57] M. Brech, J. Voit, and H. Büttner, Europhys. Lett. 12, 289 (1990).

[58] V. J. Emery, Phys. Rev. Lett. 37, 107 (1976). 
[59] J. Voit, Phys. Rev. B 47, 6740 (1993).

[60] V. Meden and K. Schönhammer, Phys. Rev. B 46, 15753 (1992).

[61] H. C. Fogedby, J. Phys. C 9, 3757 (1976).

[62] J. Voit, in the Proceedings of the NATO Advanced Research Workshop on The Physics and Mathematical Physics of the Hubbard Model, San Sebastian, October 3-8, 1993, edited by D. Baeriswyl, D. K. Campbell, J. M. P. Carmelo, F. Guinea, and E. Louis; Plenum Press, New York (1995).

[63] K. Schönhammer and V. Meden, Phys. Rev. B 48, 11390 (1993).

[64] J. Voit, Synth. Met. 70, 1015 (1995).

[65] K. Penc and J. Sólyom, Phys. Rev. B 44, 12690 (1991).

[66] C. Di Castro and W. Metzner, Phys. Rev. Lett. 67, 3852 (1991);

[67] D. K. K. Lee and Y. Chen, J. Phys. A 21, 4155 (1988).

[68] D. Schmeltzer, Phys. Rev. B 43, 8650 (1991).

[69] C. Mudry and E. Fradkin, Phys. Rev. B 50, 11409 (1994).

[70] S.-K. Ma, Modern Theory of Critical Phenomena, Benjamin/Cummings Publ. Comp., Reading, MA, 1976.

[71] H. W. J. Blöte, J. L. Cardy, and M. P. Nightingale, Phys. Rev. Lett. 56, 742 (1986); I. Affleck, ibid. p. 746 .

[72] D. Friedan, Z. Qiu, and S. Shenker, Phys. Rev. Lett. 52, 1575 (1984).

[73] J. Cardy, J. Phys. A 17, L385 (1984) and Nucl. Phys. B 270, [FS16], 186 (1986).

[74] A. D. Mironov and A. V. Zabrodin, Phys. Rev. Lett. 66, 534 (1991).

[75] F. D. M. Haldane, Phys. Lett. 81A, 153 (1981).

[76] S.-T. Chui and P. A. Lee, Phys. Rev. Lett. 35, 325 (1975).

[77] J. M. Kosterlitz and D. J. Thouless, J. Phys. C 6, 1181 (1973); J. M. Kosterlitz, J. Phys. C 7, 1046 (1974).

[78] J. Voit, J. Phys. C 21, L1141 (1988).

[79] T. Giamarchi and H. J. Schulz, Phys. Rev. B 39, 4620 (1989).

[80] J. L. Black and V. J. Emery, Phys. Rev. B 23, 429 (1981).

[81] H. A. Bethe, Z. Phys. 71, 205 (1931). 
[82] J. Hubbard, Proc. Roy. Soc. A 240, 539 (1957); 243, 336 (1958); 276, 238 (163).

[83] E. H. Lieb and F. Y. Wu, Phys. Rev. Lett. 20, 1445 (1968).

[84] P. A. Bares and G. Blatter, Phys. Rev. Lett. 64, 2567 (1990).

[85] B. Sutherland, Phys. Rev. B 12, 3795 (1975).

[86] P. Schlottmann, Phys. Rev. B 36, 5177 (1987).

[87] H. Bergknoff and H. B. Thacker, Phys. Rev. D 19, 3666 (1979).

[88] E. Lieb and W. Liniger, Phys. Rev. 130, 1616 (1963).

[89] J. Hubbard, Phys. Rev. B 17, 494 (1978).

[90] H. J. Schulz, Phys. Rev. Lett. 71, 1864 (1993).

[91] S. Kivelson, W.-P. Su, J. R. Schrieffer, and A. J. Heeger, Phys. Rev. Lett. 58, 1899 (1987).

[92] A. Painelli and A. Girlando, in Ref. [93].

[93] Interacting Electrons in Reduced Dimensions, ed. by D. Baeriswyl and D. K. Campbell, Plenum Press, New York (1989).

[94] D. K. Campbell, J. T. Gammel, and E. Y. Loh Jr. Phys. Rev. B 42, 475 (1990).

[95] F. Buzatu, Phys. Rev. B 49, 10176 (1994).

[96] J. E. Hirsch, Physica C 158, 326 (1989).

[97] F. C. Zhang and T. M. Rice, Phys. Rev. B 37, 3759 (1988); A. Fortunelli and A. Painelli, Sol. State Comm. 89771 (1994).

[98] K.-J.-B. Lee and P. Schlottmann, Phys. Rev. Lett. 63, 2299 (1989); P. Schlottmann, Phys. Rev. B 43, 3101 (1991).

[99] F. Gebhard and A. E. Ruckenstein, Phys. Rev. Lett. 68, 244 (1992); F. Gebhard, A. Girndt, and A. E. Ruckenstein, Phys. Rev. B 49, 10926 (1994).

[100] P. Nozières, Lecture Notes, Collège de France, Paris, 1991/92.

[101] H. Shiba, Phys. Rev. B 6, 930 (1972).

[102] M. Takahashi, Prog. Theor. Phys. 47, 69 (1972).

[103] T. B. Bahder and F. Woynarovich, Phys. Rev. B 33, 2114 (1986); K. Lee and P. Schlottmann, Phys. Rev. B 38, 11566 (1988).

[104] A. A. Ovchinnikov, Sov. Phys. JETP 30, 1160 (1970). 
[105] C. F. Coll III, Phys. Rev. B 9, 2150 (1974).

[106] F. D. M. Haldane and Yuhai Tu, unpublished.

[107] M. Ogata and H. Shiba, Phys. Rev. B 41, 2326 (1990).

[108] J. Carmelo and D. Baeriswyl, Phys. Rev. B 37, 7541 (1988).

[109] J. E. Hirsch and D. J. Scalapino, Phys. Rev. B 27, 7169 (1983) and 29, 5554 (1984).

[110] S. Mazumdar and A. N. Bloch, Phys. Rev. Lett. 50, 207, (1983); S. Mazumdar, S. N. Dixit, and A. N. Bloch, Phys. Rev. B 30, 4842 (1984); S. Mazumdar and S. N. Dixit, ibid. 34, 3683 (1986).

[111] K. C. Ung, S. Mazumdar, and D. Toussaint, Phys. Rev. Lett. 73, 2603 (1994).

[112] G. Goméz-Santos, Phys. Rev. Lett. 70, 3780 (1993).

[113] S. Sorella and M. Parinello, in Ref. 93.

[114] S. Sorella, E. Tosatti, S. Baroni, R. Car, and M. Parinello, Int. J. Mod. Phys. B 1, 993 (1988).

[115] C. Bourbonnais, H. Nélisse, A. Reid, and A.-M. S. Tremblay, Phys. Rev. B 40, 2297 (1989).

[116] M. Imada and Y. Hatsugai, J. Phys. Soc. Jpn. 58, 3752 (1989).

[117] S. Sorella, A. Parola, M. Parinello, and E. Tosatti, Europhys. Lett. 12, 721 (1990).

[118] T. A. Kaplan, P. Horsch, and J. Borysowicz, Phys. Rev. B 35, 1877 (1987); R. R. P. Singh, M. E. Fisher, and R. Shankar, Phys. Rev. B 39, 2562 (1989).

[119] A. Parola and S. Sorella, Phys. Rev. Lett. 64, 1831 (1990).

[120] S. Sorella and A. Parola, J. Phys. CM 4, 3589 (1992).

[121] A. Parola and S. Sorella, Phys. Rev. B 45, 13156 (1992).

[122] Y. Ren and P. W. Anderson, Phys. Rev. B 48, 16662 (1993).

[123] A. G. Izergin, V. E. Korepin, and N. Yu. Reshetikhin, J. Phys. A 22, 2615 (1989);

F. Woynarovich, ibid., p. 4243; H. Frahm and N.-C. Yu, ibid. 23, 2115 (1990).

[124] H. Frahm and V. E. Korepin, Phys. Rev. B 42, 10553 (1990).

[125] N. Kawakami and S.-K. Yang, Phys. Lett. A 148, 359 (1990).

[126] K. Penc and J. Sólyom, Phys. Rev. B 47, 6273 (1993).

[127] H. Frahm and V. E. Korepin, Phys. Rev. B 43, 5653 (1991). 
[128] R. Preuss, A. Muramatsu, W. von der Linden, P. Dieterich, F. F. Assaad, and W. Hanke, Phys. Rev. Lett. 73, 732 (1994).

[129] N. Kawakami and S.-K. Yang, Phys. Rev. Lett. 65, 2309, (1990) and J. Phys. CM 3, 5983 (1991).

[130] M. Ogata, M. Luchini, S. Sorella, and F. F. Assaad, Phys. Rev. Lett. 66, 2388 (1991).

[131] C. S. Hellberg and E. J. Mele, Phys. Rev. B 48, 646 (1993).

[132] C. S. Hellberg and E. J. Mele, Phys. Rev. B 44, 1360 (1991).

[133] C. S. Hellberg and E. J. Mele, Phys. Rev. Lett. 67, 2080 (1991).

[134] M. C. Gutzwiller, Phys. Rev. Lett. 10, 159 (1963).

[135] F. Gebhard and D. Vollhardt, Phys. Rev. Lett. 59, 1472 (1987).

[136] B. Sutherland, Phys. Rev. A 4, 2019 (1971) and 5, 1372 (1972); F. D. M. Haldane, Phys. Rev. Lett. 60, 635 (1988); B. S. Shastry, ibid., p. 639.

[137] N. Kawakami and P. Horsch, Phys. Rev. Lett. 68, 3110 (1992).

[138] C. S. Hellberg and E. J. Mele, Phys. Rev. Lett. 68, 3111 (1992).

[139] B. Fourcade and G. Spronken, Phys. Rev. B 29, 5089 and 5096, (1984); J. E. Hirsch, Phys. Rev. Lett. 53, 2327 (1984); H. Q. Lin and J. E. Hirsch, Phys. Rev. 33, 8155 (1986); J. W. Cannon and E. Fradkin, Phys. Rev. B 41, 9435 (1990); J. W. Cannon, R. T. Scalettar, and E. Fradkin, Phys. Rev. B 44, 5995 (1991).

[140] A. Luther, Phys. Rev. B 14, 2153 (1976).

[141] A. Luther, Phys. Rev. B 15, 403 (1977).

[142] F. Mila and X. Zotos, Europhys. Lett. 24, 133 (1993).

[143] K. Penc and F. Mila, Phys. Rev. B 49, 9670 (1994).

[144] K. Sano and Y. Ono, J. Phys. Soc. Jpn. 63, 1250 (1994).

[145] K. Kuroki, K. Kusakabe, and H. Aoki, Phys. Rev. B 50, 575 (1994).

[146] N. D. Mermin and H. Wagner, Phys. Rev. Lett. 17, 1133 (1966); P. C. Hohenberg, Phys. Rev. 158, 383 (1967).

[147] S. Takada, Prog. Theor. Phys. 54, 1039 (1975).

[148] P. Bak and R. Bruinsma, Phys. Rev. Lett. 49, 249 (1982); G. V. Uimin and V. L. Pokrovsky, J. Phys. (Paris) Lett. 44, L865 (1983); L. A. Bol'shov, V. L. Pokrovsky, and G. V. Uimin, J. Stat. Phys. 38, 191 (1985). 
[149] W.-P. Su, J. R. Schrieffer, and A. J. Heeger, Phys. Rev. Lett. 42, 1698 (1979) and Phys. Rev. B 22, 2099 (1980).

[150] A. J. Heeger, S. Kivelson, J. R. Schrieffer, and W.-P. Su, Rev. Mod. Phys. 60, 781 (1988).

[151] T. Holstein, Ann. Phys. 8, 325 and 343 (1959).

[152] D. Feinberg, S. Chiuchi, and F. de Pasquale, Int. J. Mod. Phys. B 4, 1317 (1990).

[153] J. M. Ginder and A. J. Epstein, Phys. Rev. B 41, 10674 (1990); D. Baranowski, H. Büttner, and J. Voit, ibid. 45, 10990 (1992) and 47, 15472 (1993).

[154] J. Voit and H. J. Schulz, Phys. Rev. B 34, 7429 (1986), 36, 968 (1985), and 37, 10068 (1988); L. G. Caron and C. Bourbonnais, Phys. Rev. B 29, 4230 (1984); C. Bourbonnais and L. G. Caron, J. Phys. (Paris) 50, 2751 (1989).

[155] S. Engelsberg and B. B. Varga, Phys. Rev. 136, A1583 (1964); J. Voit and H. J. Schulz, Molec. Cryst. Liq. Cryst. 119, 449 (1985). An error in the treament of acoustic phonons in this paper has been corrected by Y. Chen, D. K. K. Lee, and M. U. Luchini, Phys. Rev. B 38, 8497 (1988).

[156] D. Loss and T. Martin, Phys. Rev. B 50, 12160 (1994).

[157] J. Voit, Phys. Rev. Lett. 64, 323 (1990).

[158] G. T. Zimanyi, S. A. Kivelson, and A. Luther, Phys. Rev. Lett. 60, 2089 (1988).

[159] J. Voit, Synth. Met. 27, A41 (1988).

[160] V. Meden, K. Schönhammer, and O. Gunnarsson, Phys. Rev. B 50, 11179 (1994).

[161] T. Giamarchi and H. J. Schulz, Europhys. Lett. 3, 1287 (1987), and Phys. Rev. B 37, 325 (1988).

[162] S.-T. Chui and J. W. Bray, Phys. Rev. B 16, 1329 (1977); W. Apel, J. Phys. C 15, 1973 (1982); Y. Suzumura and H. Fukuyama, J. Phys. Soc. Jpn. 52, 2870 (1983) and 53, 3918 (1984).

[163] A. A. Abrikosov and J. A. Ryzhkin, Adv. Phys. 27, 147 (1978).

[164] P. W. Anderson, J. Phys. Chem. Sol. 11, 26 (1959).

[165] H. Fukuyama and P. A. Lee, Phys. Rev. B 17, 535 (1978).

[166] M. Ogata and P. W. Anderson, Phys. Rev. Lett. 70, 3087, (1993).

[167] H. Basista, D. A. Bonn, T. Timusk, J. Voit, D. Jérôme, and K. Bechgaard, Phys. Rev. B 42, 4088 (1990). 
[168] J. M. P. Carmelo and P. Horsch, Phys. Rev. Lett. 68, 871 (1992).

[169] C. L. Kane and M. P. A. Fisher, Phys. Rev. Lett. 68, 1220 (1992), Phys. Rev. B 46, 7268 and 15233 (1992).

[170] W. Apel and T. M. Rice, Phys. Rev. B 26, 7063 (1982).

[171] A. Furusaki and N. Nagaosa, Phys. Rev. B 47, 4631 (1993).

[172] K. A. Matveev, D. Yue, and L. I. Glazman, Phys. Rev. Lett. 71, 3351 (1993).

[173] A. Furusaki and N. Nagaosa, Phys. Rev. B 47, 3827 (1993).

[174] X. G. Wen, Phys. Rev. Lett. 64, 2206 (1990), Phys. Rev. B 41, 12838 (1990) and 43, 11025 (1991).

[175] K. Moon, H. Yi, C. L. Kane, S. M. Girvin, and M. P. A. Fisher, Phys. Rev. Lett. 71, 4381 (1993).

[176] M. Fabrizio, A. O. Gogolin, and S. Scheidl, Phys. Rev. Lett. 72, 2235 (1994).

[177] H. Fukuyama, H. Kohno, and R. Shirasaki, J. Phys. Soc. Jpn. 62, 1109 (1993).

[178] M. Ogata and H. Fukuyama, Phys. Rev. Lett. 73, 468 (1994).

[179] P. A. Lee and T. V. Ramakrishnan, Rev. Mod. Phys. 57, 287 (1985).

[180] N. F. Mott and W. D. Twose, Adv. Phys. 10, 107 (1961).

[181] J. Carmelo and A. A. Ovchinnikov, J. Phys. CM 3, 757 (1991).

[182] J. Carmelo, P. Horsch, P. A. Bares, and A. A. Ovchinnikov, Phys. Rev. B 44, 9967 (1991).

[183] J. M. P. Carmelo, P. Horsch, and A. A. Ovchinnikov, Phys. Rev. B 45, 7899 and 46, 14728 (1992).

[184] J. M. P. Carmelo, A. H. Castro Neto, and D. K. Campbell, Phys. Rev. Lett. 73, 926 and Phys. Rev. B 50, 3667 and 3683 (1994).

[185] H. J. Schulz, in Strongly Correlated Electronic Materials: The Los Alamos Symposium 1993, ed. by K. S. Bedell et al., Addison-Wesley, Reading, 1994, p. 187.

[186] A. Luther and V. J. Emery, Phys. Rev. Lett. 33, 589 (1974); P. A. Lee, Phys. Rev. Lett. 34, 1247 (1973).

[187] V. J. Emery, A. Luther, and I. Peschel, Phys. Rev. B 13, 1272 (1976).

[188] S. Coleman, Phys. Rev. D 11, 2088 (1975).

[189] M. Fowler and X. Zotos, Phys. Rev. B 24, 2634 (1981). 
[190] E. K. Sklyanin, L. A. Takhtadzhyan, and L. D. Faddeev, Theor. Mat. Phys. 40, 688 (1979).

[191] F. D. M. Haldane, J. Phys. A 15, 507 (1982).

[192] T. Giamarchi and H. J. Schulz, Phys. Rev. B 33, 2066 (1986) and J. Phys. (Paris) 49, 819 (1988).

[193] H. J. Schulz, Phys. Rev. B 22, 5274 (1980).

[194] M. Gulácsi and K. S. Bedell, Phys. Rev. Lett. 72, 2765 (1994).

[195] V. L. Pokrovsky and A. L. Talapov, Phys. Rev. Lett. 42, 65 (1979).

[196] V. J. Emery, Phys. Rev. Lett. 65, 1076 (1990).

[197] C. A. Stafford and A. J. Millis, Phys. Rev. B 48, 1409, (1993).

[198] H. Eskes and A. Oleś, Phys. Rev. Lett. 73, 1279 (1994).

[199] P. M. Chaikin, R. L. Greene, S. Etemad, and E. Engler, Phys. Rev. B 13, 1627 (1976).

[200] E. B. Kolomeisky, Phys. Rev. B 47, 6193 (1993).

[201] J. P. Straley and E. B. Kolomeisky, Phys. Rev. B 48, 1378 (1993).

[202] B. Horovitz, T. Bohr, J. M. Kosterlitz, and H. J. Schulz, Phys. Rev. 28, 6596 (1983).

[203] W. Götze and P. Wölfle, Phys. Rev. B 6, 1226 (1972).

[204] T. Giamarchi, Phys. Rev. B 46, 342 (1992).

[205] M. Mori, H. Fukuyama, and M. Imada, J. Phys. Soc. Jpn. 63, 1639 (1994).

[206] H. Pang, S. Liang, and J. F. Annett, Phys. Rev. Lett. 71, 4377 (1993).

[207] E. B. Kolomeisky, Phys. Rev. B 48, 4998 (1993).

[208] M. Ma, Phys. Rev. B 26, 5097 (1982).

[209] P. Horsch and W. Stephan, Phys. Rev. B 48, 10 595, (1993).

[210] W. F. Brinkman and T. M. Rice, Phys. Rev. B 2, 1324 (1970).

[211] K. J. von Szczepanski, P. Horsch, W. Stephan, and M. Ziegler, Phys. Rev. B 41, 2017 (1990); M. Ziegler and P. Horsch, in Dynamics of Magnetic Fluctuations in High-Temperature Superconductors, ed. by G. Reiter and P. Horsch, Plenum Press, New York, 1991, p.329.

[212] K. Penc and J. Sólyom, Phys. Rev. B 41, 704 (1990). 
[213] A. A. Nersesyan, Phys. Lett. A 153, 49 (1991).

[214] G. Montambaux, M. Héritier, and P. Lederer, Phys. Rev. B 33, 7777 (1986).

[215] K. A. Muttalib and V. J. Emery, Phys. Rev. Lett. 57, 1370 (1986).

[216] I. Affleck and J. B. Marston, Phys. Rev. B 37, 3774 (1988); A. B. Harris, T. C. Lubensky, and E. J. Mele, Phys. Rev. B 40, 2631 (1989).

[217] C. M. Varma and A. Zawadowski, Phys. Rev. B 32, 7399 (1985).

[218] P. Nozières, Ann. Phys. Fr. 10, 19 (1985).

[219] L. G. Caron and C. Bourbonnais, Europhys. Lett. 11, 473 (1990).

[220] V. J. Emery, Phys. Rev. Lett. 58, 2794 (1987); C. M. Varma, S. Schmitt-Rink, and E. Abrahams, Sol. State Comm. 62, 681 (1987).

[221] L. D. Landau and E. M. Lifshitz, Statistical Physics, Pergamon Press, London (1959), p. 482.

[222] V. N. Prigodin and Yu. A. Firsov, Sov. Phys. JETP 49, 369 and 813 (1979).

[223] P. W. Anderson, Phys. Rev. Lett. 67, 3844 (1991).

[224] R. A. Klemm and H. Gutfreund, Phys. Rev. B 14, 1086 (1976).

[225] P. A. Lee, T. M. Rice, and R. A. Klemm, Phys. Rev. B 15, 2984, (1977).

[226] L. P. Gor'kov and I. E. Dzyaloshinskii, Sov. Phys. JETP 40, 198 (1975).

[227] W. A. Little, Phys. Rev. 134, A1416 (1964).

[228] S. Barišić, J. Phys. (Paris) 44, 185 (1983); S. Botrić and S. Barišić, ibid. 45, 185 (1984).

[229] X. G. Wen, Phys. Rev. B 42, 6623 (1990).

[230] F. V. Kusmartsev, A. Luther, and A. Nersesyan, JETP Lett. 55, 724 (1992); V. Yakovenko, ibid. 56, 510 (1992).

[231] S. Brazovskii and V. Yakovenko, J. Phys. (Paris) Lett. 46, L-111 (1985); Sov. Phys. JETP 62, 1340 (1985); J. Phys. (Paris) 47, 175 (1986).

[232] C. Bourbonnais and L. G. Caron, Europhys. Lett. 5, 209 (1988).

[233] D. G. Clarke, S. P. Strong, and P. W. Anderson, Phys. Rev. Lett. 72, 3218 (1994).

[234] M. Fabrizio and A. Parola, Phys. Rev. Lett. 70, 226 (1993).

[235] M. Fabrizio, Phys. Rev. B 48, 15838 (1993). 
[236] A. M. Finkel'stein and A. I. Larkin, Phys. Rev. B 47, 10461 (1993).

[237] C. Castellani, D. di Castro, and W. Metzner, Phys. Rev. Lett. 69, 1703 (1992).

[238] M. Fabrizio, A. Parola, and E. Tosatti, Phys. Rev. B 46, 3159 (1992).

[239] R. M. Noack, S. R. White, and D. J. Scalapino, Phys. Rev. Lett. 73, 882 (1994).

[240] Y. Asai, Phys. Rev. B 50, 6519 (1994).

[241] T. M. Rice, S. Gopalan, and M. Sigrist, Europhys. Lett. 23, 445 (1993).

[242] S. P. Strong and A. J. Millis, Phys. Rev. Lett. 69, 2419 (1992); T. Barnes, E. Dagotto, J. Riera, and E. S. Swanson, Phys. Rev. B 47, 3196 (1993); S. Gopalan, T. M. Rice, and M. Sigrist, Phys. Rev. B 49, 8901 (1994).

[243] D. V. Khveshchenko, Phys. Rev. B 50, 386 (1994).

[244] H. Tsunetsugu, M. Troyer, and T. M. Rice, Phys. Rev. B 49, 16078 (1994).

[245] J. Voit and E. J. Mele, Synth. Met. 43, 3911 (1991).

[246] D. Poilblanc, H. Tsunetsugu, and T. M. Rice, Phys. Rev. B 50, 6511 (1994).

[247] J. A. Riera, Phys. Rev. B 49, 3629 (1994).

[248] D. Boies, C. Bourbonnais, and A.-M. S. Tremblay, Phys. Rev. Lett. 74, 968 (1995).

[249] P. Kopietz, V. Meden, and K. Schönhammer, Phys. Rev. Lett. 74, 2997 (1995).

[250] R. Valentí and C. Gros, Phys. Rev. Lett. 68, 2402 (1992) and (E) 69, 996 (1992);

C. Gros and R. Valentí, Mod. Phys. Lett. B 7, 119 (1993).

[251] Y. C. Chen and T. K. Lee, Z. Phys. B 95, 5 (1994).

[252] W. O. Putikka, R. L. Glenister, R. R. P. Singh, and H. Tsunetsugu, Phys. Rev. Lett. 73, 170 (1994).

[253] Y. C. Chen, A. Moreo, F. Ortolani, E. Dagotto, and T. K. Lee, Phys. Rev. B 50, 655 (1994); C. Gros and R. Valentí, ibid., p. 11313; T. Tohyama, P. Horsch, and S. Maekawa, Phys. Rev. Lett. 74, 980 (1995).

[254] C. Castellani, C. Di Castro, and W. Metzner, Phys. Rev. Lett. 72, 316 (1994).

[255] T. Holstein, R. E. Norton, and P. Pincus, Phys. Rev. B 8, 2649 (1973); M. Yu. Reizer, Phys. Rev. B 39, 1602 (1989) and 4011571 (1989); F. Guinea and G. Zimanyi, Phys. Rev. B 47, 501 (1993); P. A. Bares and X.-G. Wen, Phys. Rev. B 48, 8636 (1993); D. V. Khveshchenko, R. Hlubina, and T. M. Rice, Phys. Rev. B 48, 10766 (1993); R. Hlubina, Phys. Rev. B 50, 8252 (1994). 
[256] A. Luther, Phys. Rev. B. 19, 320 (1979); R. Shankar, Physica A 177, 530 (1991) and Rev. Mod. Phys. 66, 129 (1994); A. Houghton and J. B. Marston, Phys. Rev. B 48, 7790 (1993); A. Houghton, H.-J. Kwon, and J. B. Marston, Phys. Rev. B 50, 1351 (1994); A. H. Castro Neto and E. Fradkin, Phys. Rev. Lett. 72, 1393 (1994) and Phys. Rev. B 49, 10877 (1994).

[257] The Quantum Hall Effect, ed. by R. E. Prange and S. M. Girvin, Springer Verlag, New York, 1987.

[258] K. von Klitzing, G. Dorda, and M. Pepper, Phys. Rev. Lett. 45, 494 (1980).

[259] D. C. Tsui, H. L. Sörmer, and A. Gossard, Phys. Rev. Lett. 48, 1559 (1982).

[260] B. I. Halperin, Phys. Rev. B 25, 2185 (1982).

[261] M. Büttiker, Phys. Rev. B 38, 9375 (1988).

[262] For a review, see X.-G. Wen, Int. J. Mod. Phys. B 6, 1711 (1992).

[263] M. Stone, Ann. Phys. (NY) 207, 38 (1991) and Int. J. Mod. Phys. B 5, 509 (1991).

[264] M. D. Johnson and A. H. MacDonald, Phys. Rev. Lett. 67, 2060 (1991).

[265] D. Jérôme and H. J. Schulz, in Extended Linear Chain Compounds, Vol. 2, edited by J. S. Miller, Plenum Press, New York (1982).

[266] J. P. Pouget, S. K. Khanna, F. Denoyer, R. Comès, A. F. Garito, and A. J. Heeger, Phys. Rev. Lett. 37, 437 (1976).

[267] S. Kagoshima, T. Ishiguro, and H. Anzai, J. Phys. Soc. Japan 41, 2061 (1976).

[268] S. Klotz, J. S. Schilling, M. Weger, and K. Bechgaard, Phys. Rev. B 38, 5878 (1988).

[269] T. Takahashi, D. Jérôme, F. Masin, J. M. Fabre, and L. Giral, J. Phys. C 17, 3777 (1984).

[270] J. B. Torrance, in Ref. [25].

[271] J. P. Pouget, R. Comès, A. J. Epstein, and J. S. Miller, Molec. Cryst. Liq. Cryst. 85, 1593 (1982).

[272] J. Skov Pedersen and K. Carneiro, Rep. Prog. Phys. 50, 995 (1987).

[273] S. Tomić, J. R. Cooper, and K. Bechgaard, Phys. Rev. Lett. 62, 462 (1989).

[274] D. Jérôme, A. Mazaud, M. Ribault, and K. Bechgaard, J. Phys. Lett. (Paris) 41, L95 (1980).

[275] S. S. P. Parkin, M. Ribault, D. Jérôme, and K. Bechgaard, J. Phys. C 14, 5305 (1981). 
[276] L. Forró, J. R. Cooper, B. Rothaemel, J. S. Schilling, M. Weger, and K. Bechgaard, Solid State Comm. 60, 11 (1986).

[277] R. Bozio, M. Meneghetti, D. Pedron, and C. Pecile, Synth. Met. 27, B129 (1988).

[278] R. Bozio, M. Meneghetti, and C. Pecile, J. Chem. Phys. 76, 5785 (1982).

[279] M. J. Rice, Phys. Rev. Lett. 37, 36 (1976).

[280] C. Bourbonnais, F. Creuzet, D. Jérome, K. Bechgaard, and A. Moradpour, J. Phys (Paris) Lett. 45, L-755 (1984).

[281] P. Wzietek, F. Creuzet, C. Bourbonnais, D. Jérôme, and A. Moradpour, J. Phys. (Paris) I 3, 171 (1993).

[282] C. Bourbonnais, in Ref. [25].

[283] C. Bourbonnais, J. Phys. (Paris) I 3, 143 (1993).

[284] L. Ducasse, M. Abderraba, J. Hoarau, M. Pesquer, B. Gallois, and J. Gaultier, J. Phys. C 19, 3805 (1986).

[285] G. Soda, D. Jérôme, M. Weger, J. M. Fabre, and L. Giral, Solid State Comm. 18, 1417 (1976).

[286] G. Soda, D. Jérôme, M. Weger, J. Alizon, J. Gallice, H. Robert, J. M. Fabre, and L. Giral, J. Phys. (Paris) 38, 931 (1977).

[287] P. C. Stein, A. Moradpour, and D. Jérôme, J. Phys. Lett. (Paris) 46, 241 (1985).

[288] B. Dardel, D. Malterre, M. Grioni, P. Weibel, Y. Baer, J. Voit, and D. Jérôme, Europhys. Lett. 24, 687 (1993).

[289] F. Mila and K. Penc, Synth. Met. 70, 997 (1995).

[290] Electronic Properties of Inorganic Quasi-One-Dimensional Compounds, vol. 1 and 2, edited by P. Monceau, D. Reidel Publ. Comp., Dordrecht (1985).

[291] P. A. Lee, T. M. Rice, and P. W. Anderson, Phys. Rev. Lett. 31, 462 (1973).

[292] C. Schlenker and J. Dumas, in Crystal Chemistry and Properties of Materials with Quasi-One-Dimensional Structures, ed. by J. Rouxel, D. Reidel Publ. Comp., Dordrecht, 1986, p. 135.

[293] B. Dardel, D. Malterre, M. Grioni, P. Weibel, Y. Baer, and F. Lévy, Phys. Rev. Lett. 67, 3144 (1991); J.-Y. Veuillen, R. C. Cinti, and E. Al Khoury Nemeh, Europhys. Lett. 3, 355 (1987).

[294] Y. Hwu, P. Alméras, M. Marsi, H. Berger, F. Lévy, M. Grioni, D. Malterre, and G. Margaritondo, Phys. Rev. B 46, 13624 (1992) 
[295] K. E. Smith, K. Breuer, M. Greenblatt, and W. McCarrol, Phys. Rev. Lett. 70, 3772 (1993).

[296] L. Degiorgi, G. Grüner, K. Kim, R. H. McKenzie, and P. Wachter, Phys. Rev. B 49, 14754 (1994).

[297] K. Kim, R. H. McKenzie, and J. W. Wilkins, Phys. Rev. Lett. 71, 4015 (1993).

[298] N. Nakamura, A. Sekiyama, H. Namatame, A. Fujimori, H. Yoshihara, T. Ohtani, A. Misu, and M. Takano, Phys. Rev. B 49, 16191 (1994).

[299] U. Meirav, M. A. Kastner, M. Heiblum, and S. J. Wind, Phys. Rev. B 40, 5871 (1989).

[300] F. P. Milliken, C. P. Umbach, and R. A. Webb, unpublished; also reported by B. Gross Levi, Physics Today 47, 21 (1994). 


\section{List of Figures}

Figure 2.1: The Peierls instability: the presence of an interaction component with wave vector $q \approx 2 k_{F}$ in a $1 \mathrm{D}$ electron gas (left) hybridizes the two Fermi points $\pm k_{F}$ and, in a mean-field description, opens a gap at the Fermi level (middle). Responsible is the logarithmic $2 k_{F}$ divergence in the particle-hole bubble (right).

Figure 2.2: Diagrams contributing to the self-energy to second order.

Figure 2.3: The Bethe-Salpeter equation. $\Gamma$ is the complete particle-hole interaction, $I$ the irreducible one. Solid lines are Green's functions.

Figure 3.1: Particle-hole excitations in 1D (left). The spectrum (right) has no lowfrequency excitations with $0 \leq|q| \leq 2 k_{F}$ unlike in higher dimensions where these states are filled in.

Figure 3.2: The Luttinger model. Dispersion (left) and forward scattering processes (right). Solid lines denote electrons propagating with $k_{F}$ and dashed lines those propagating with $-k_{F}$.

Figure 3.3: Backward $\left(g_{1 \perp}\right)$ and Umklapp $\left(g_{3 \perp}\right)$ scattering not included in the Luttinger model. Scattering particles have antiparallel spin here.

Figure 3.4: Dyson equation for the density correlation function $R_{\rho \rho}$.

Figure 3.5: Dyson equation for the polarization. $\lambda^{\rho}$ represents the bare vertex.

Figure 3.6: Luttinger model spectral function $\rho_{+}(q, \omega)$ for $q \geq 0$ and $\alpha=0.125$. The $\omega<0$-part has been multiplied by 10 for clarity.

Figure 3.7: Dynamical charge and spin structure factors of the Luttinger model. $S(q, \omega)$ and $S_{4}(q, \omega)$ are the $2 k_{F^{-}}$and $4 k_{F^{-}}$CDW structure factors, and $\chi(q, \omega)$ is the magnetic structure factor close to $2 k_{F}$.

Figure 4.1: Linearized renormalization group flow of $g_{1 \perp}$ and $K_{\sigma}$ for the backscattering Hamiltonian. The line $g_{1 \perp}^{\star}=0, K_{\sigma}^{\star} \geq 1$ is the Luttinger liquid fixed line.

Figure 4.2: Phase diagram of the one-dimensional Fermi liquid off half-filling. The system is a Luttinger liquid at $g_{1 \perp} \geq 0$ where $K_{\sigma}^{\star}=1$. Fluctuations indicated in parenthesis have the same exponents as the dominant ones but are logarithmically weaker. At $g_{1 \perp}<0$, there is a spin gap, and formally $K_{\sigma}^{\star}=0$. Here, fluctuations appearing in parenthesis diverge with a smaller power-law exponent than the dominant ones. 
Figure 4.3: Momentum distribution $n(k)$ of the quarter-filled Hubbard model in the limit $U / t \rightarrow \infty$ as calculated from the Bethe Ansatz equations (4.32) and (4.33). (Anti)periodic boundary conditions were used for $4 n+2-(4 n$-)site lattices, respectively. From ref. [107, Fig. 3 .

Figure 4.4 The correlation exponent $K_{\rho}$ of the Hubbard model as a function of bandfilling $n$ for different values of $U(U / t=1,2,4,8,16$ from top to bottom). From ref. [42], Fig. 3 .

Figure 4.5: The charge and spin velocities $v_{\rho}$ (full line) and $v_{\sigma}$ (dash-dotted lines) of the Hubbard model as a function of bandfilling for different $U / t . U / t=1,2,4,8,16$ from top to bottom for $v_{\sigma}$ and from bottom to top for $v_{\rho}$ in the left part of the Figure. From ref. [42], Fig. 1. $u_{\rho, \sigma}$ are denoted $v_{\rho, \sigma}$ in our text.

Figure 4.6: Phase diagram of the $t-J$-model determined from variational wavefunctions. "Repulsive Luttinger" stands for a Luttinger liquid with dominant SDW correlations, and "attractive Luttinger" for one with dominant TS. The spin-gap phase has dominant SS. The dashed line corresponds to $K_{\rho}=1$. From ref. [131], Fig. 1 .

Figure 4.7: Phase diagram of the extended Hubbard model at quarter-filling. I is an insulating $4 k_{F}$-CDW state, $\mathrm{M}$ the metallic, repulsive Luttinger liquid and $\mathrm{SC}$ an attractive Luttinger liquid with superconducting correlations. The dashed lines are lines of constant $K_{\rho}$. From [142], Fig. 1.

Figure 6.1: Dispersion relations of two hybridized bands and their linearized approximations. In (a) two particle-like bands hybridize. In (b) a particle-like band hybridizes with a hole-like band leading to different signs of the Fermi velocities on the same side of the dispersion. From Ref. [212], Figs. 1+2. (a) contains (a) from both Fig. 1 and Fig. 2; same applies to (b).

Figure 6.2: Cut at $k_{y}=0$ through the Brillouin zone of a system of weakly coupled chains. The shaded area indicates occupied electron states. From Ref. [15], Fig. 1.7. Their $k_{F}^{0}$ is denoted by $k_{F}^{1 D}$ in our text.

Figure 6.3: Generation of transverse hopping corrections to the propagation of correlated particle-particle or particle-hole pairs. The thick (thin) lines refer to 3D (1D) propagators in the high-energy shell eliminated by the renormalization group transformation. Time arrows and spin indices can be put depending on the (CDW, SDW, SS, TS)-operator under consideration, and the full square then denotes its effective combination of coupling constants. From Ref. [22], Fig. 9.

Figure 6.4: Phase diagram for the quarter-filled two-chain Hubbard model from renormalization group. The different phases are explained in the text. From Ref. [235, Fig. 9. 
$\rho$ in the inset is denoted by $n$ in our text.

Figure 6.5: Electronic structure for the integer quantum Hall effect on an annulus of radii $r_{1}$ and $r_{2}$. The shaded areas indicate localized states. From Ref. [260], Fig. 3.

Figure 7.1: Structure of the molecule tetramethyl-tetraselenafulvalene and schematic stacking pattern of the $(T M T S F)_{2} X$-crystals.

Figure 7.2: Real part of the optical conductivity of $T T F-T C N Q$ at $85 \mathrm{~K}$ along the chain axis for two different samples (trial 1 and 2). Notice the suppression of conductivity with respect to a Drude model at low frequency. From Ref. [167], Fig. 6.

Figure 7.3: ${ }^{77} \mathrm{Se}$-NMR spin-lattice relaxation rate $T_{1}^{-1}$ as a function of temperature in $(\mathrm{TMTSF})_{2} \mathrm{ClO}_{4}$. The different symbols denote different fields. Shown in the inset is the theoretical profile for $T_{1}^{-1}$ for two values of the exponent $\alpha_{S D W}$. $T_{X}^{1}$ is the singleparticle crossover temperature, and $E_{0}$ marks the temperature where the $2 k_{F}$-SDW becomes stronger than the $q \approx 0$-fluctuations. From Ref. [280], Fig. 1 (main figure) and 5 (a) (inset). $\eta$ is $\alpha_{S D W}$ in our text and $T_{X}$ is $T_{X}^{1}$.

Figure 7.4: HeII photoemission spectrum of $(T M T S F)_{2} P F_{6}$ at $T=50 K$. The HeIspectrum in the insert has a better statistics and clearly shows that there is no spectral weight near the Fermi surface. From Ref. [288], Figure 1.

Figure 7.5: Tunneling conductance through a constriction in a $\nu=1 / 3$ quantum Hall state as a function of temperature. Different curves refer to different voltages on the point contact forming the constriction. The Luttinger liquid prediction is $G(T) \sim T^{4}$. From the preprint of Ref. [300] and Physics Today (p.23). 


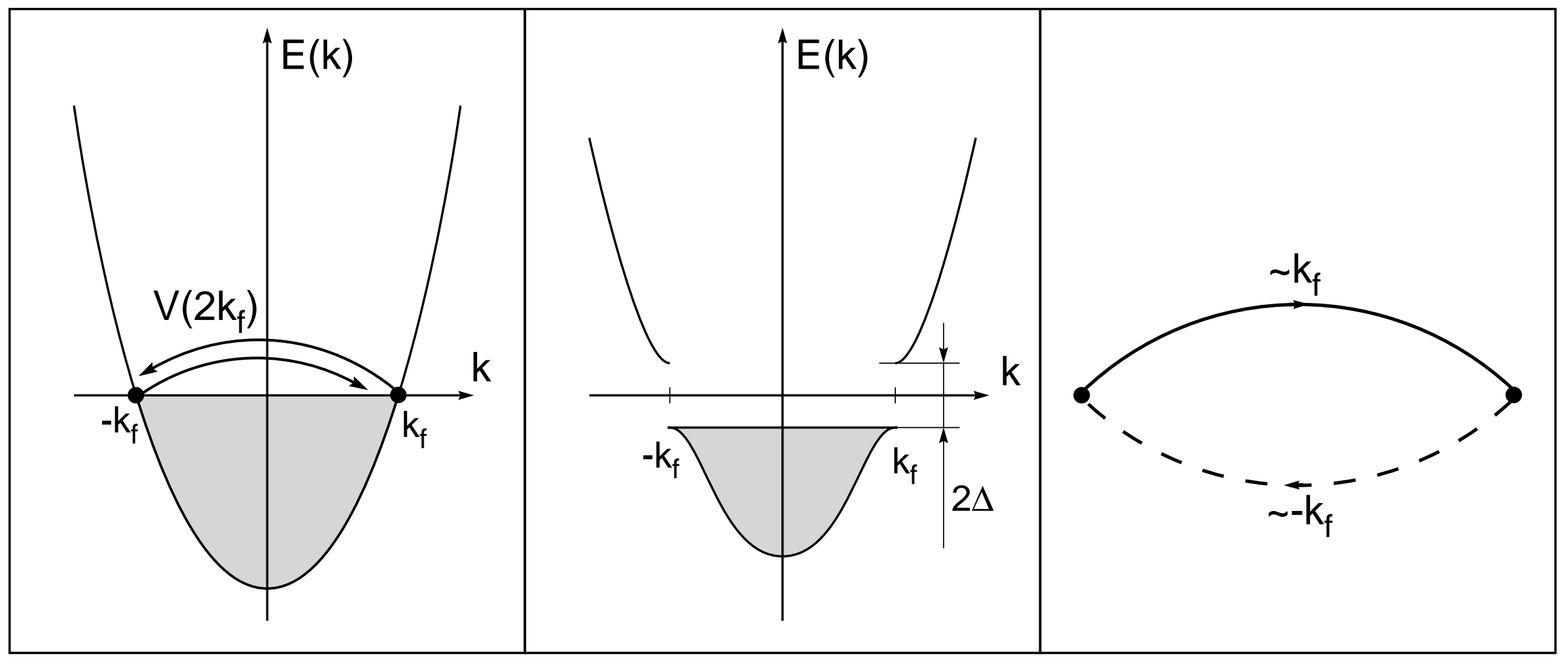



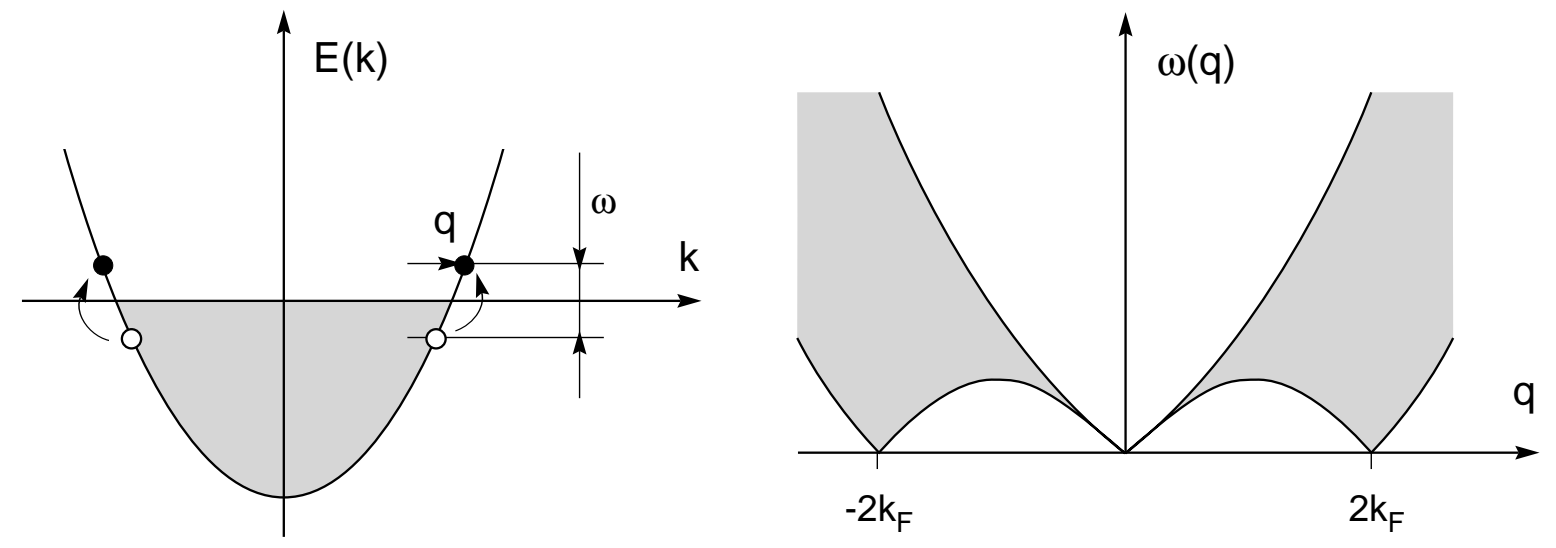


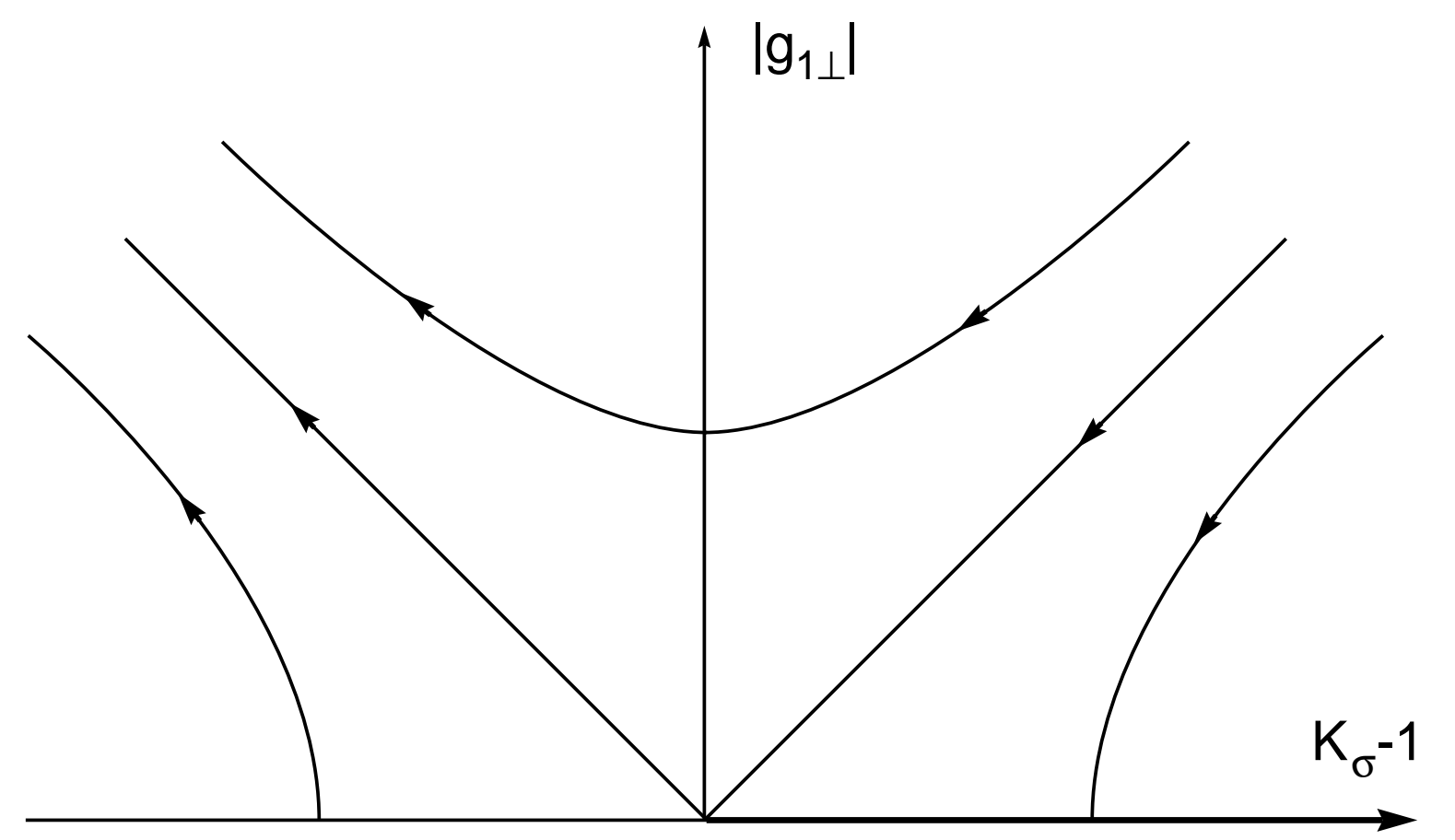




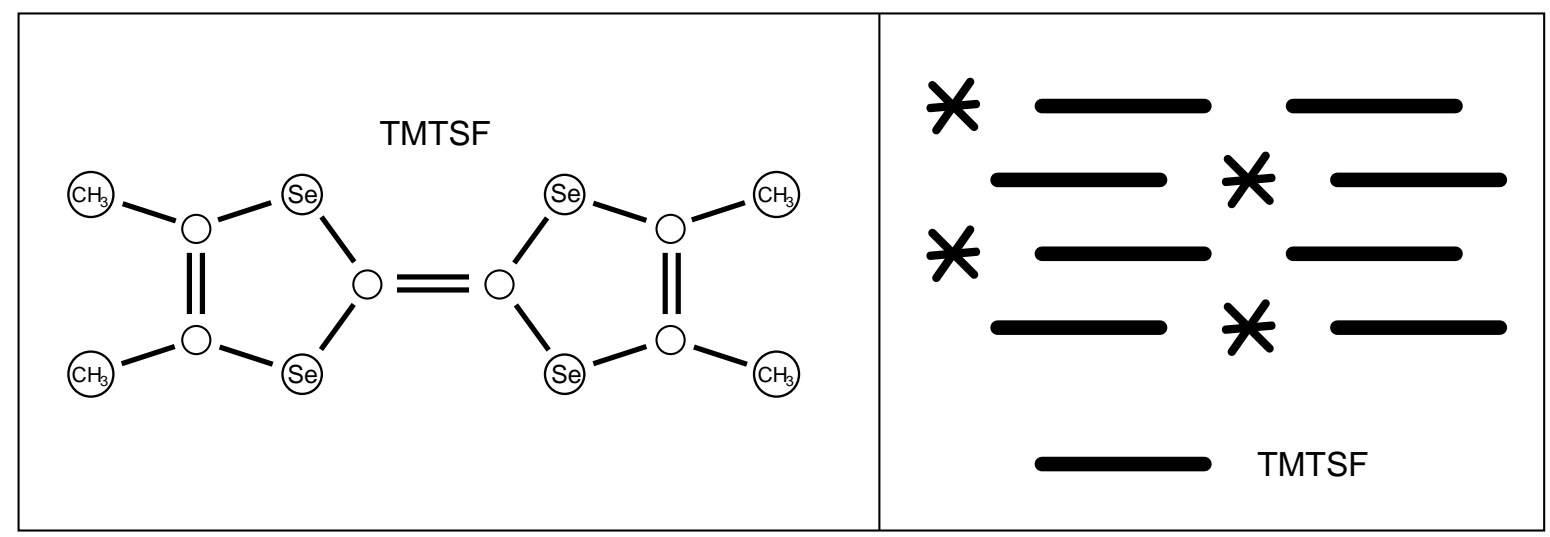




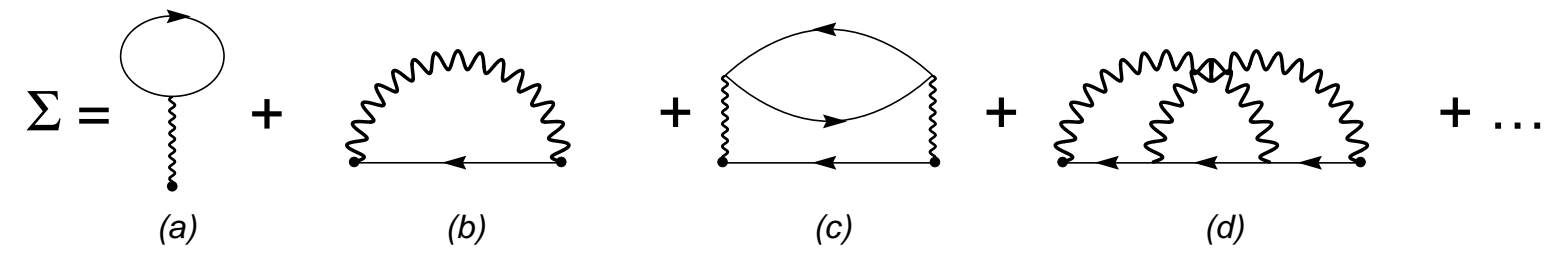



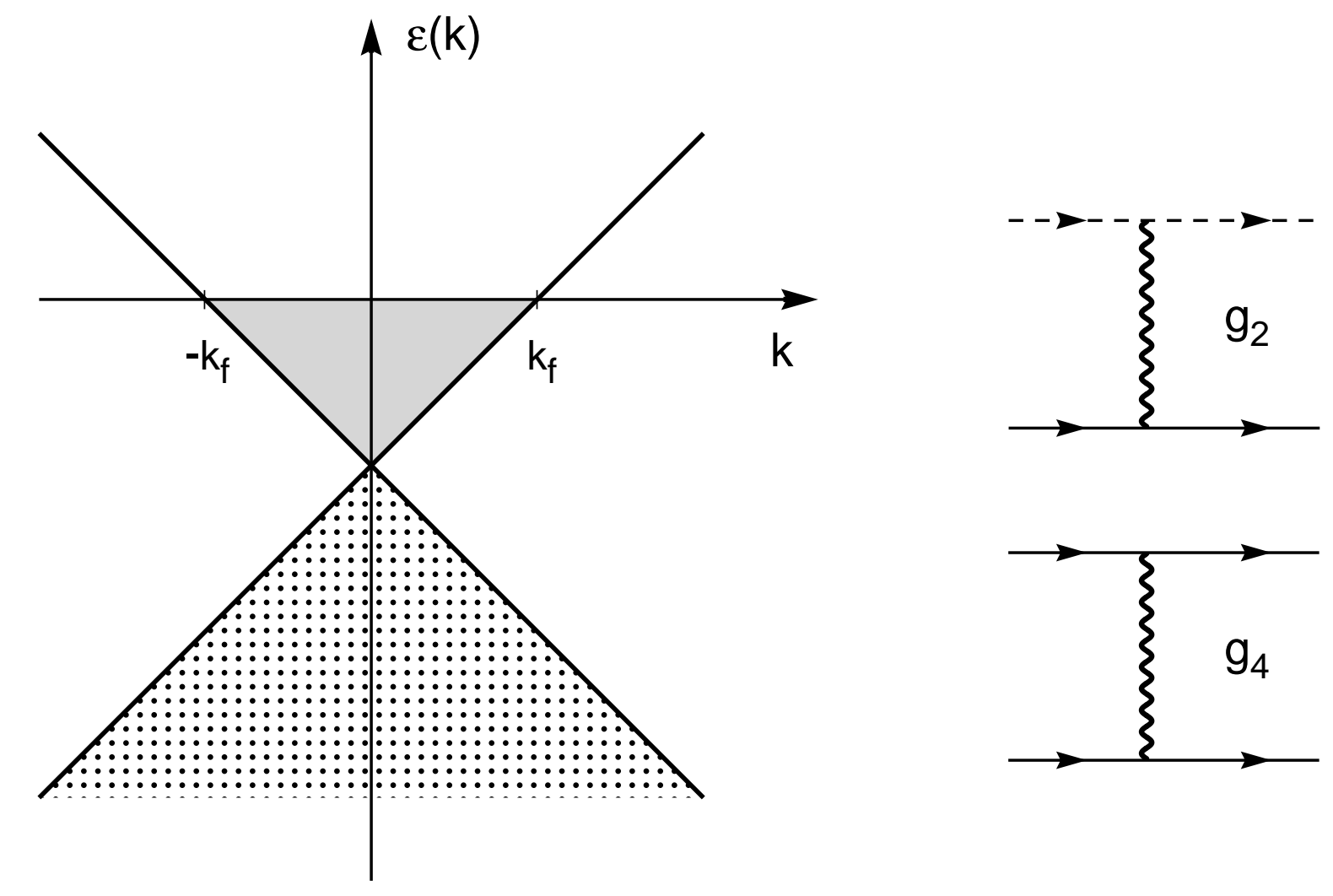


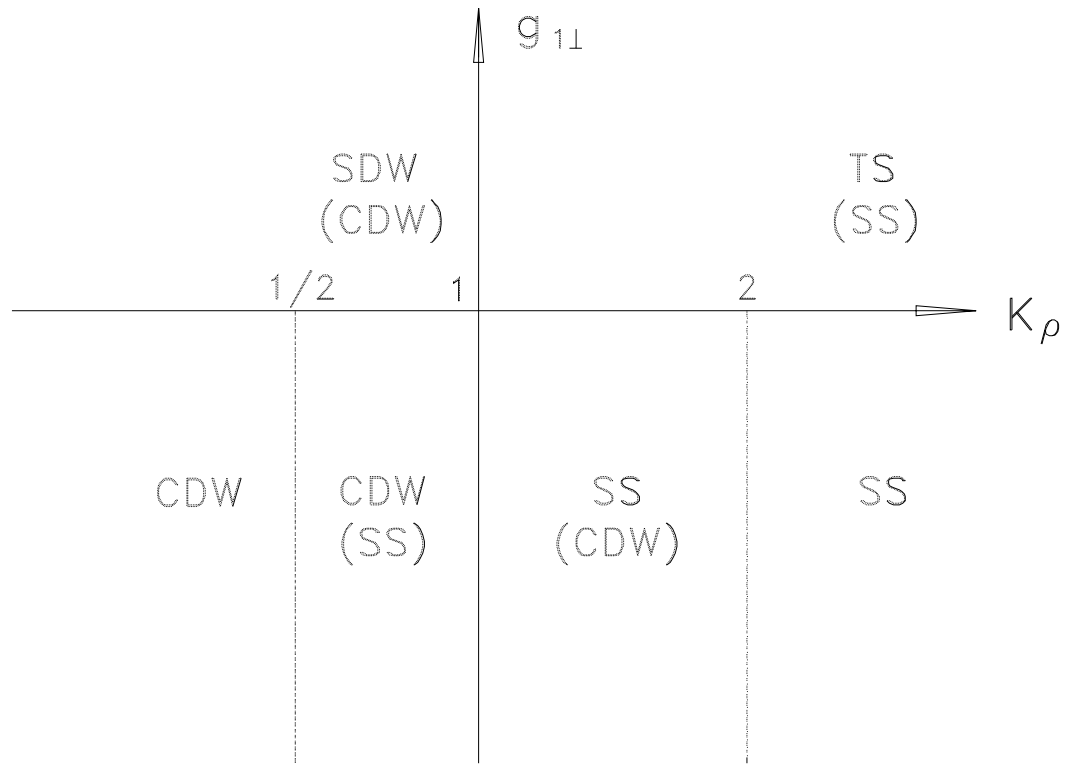



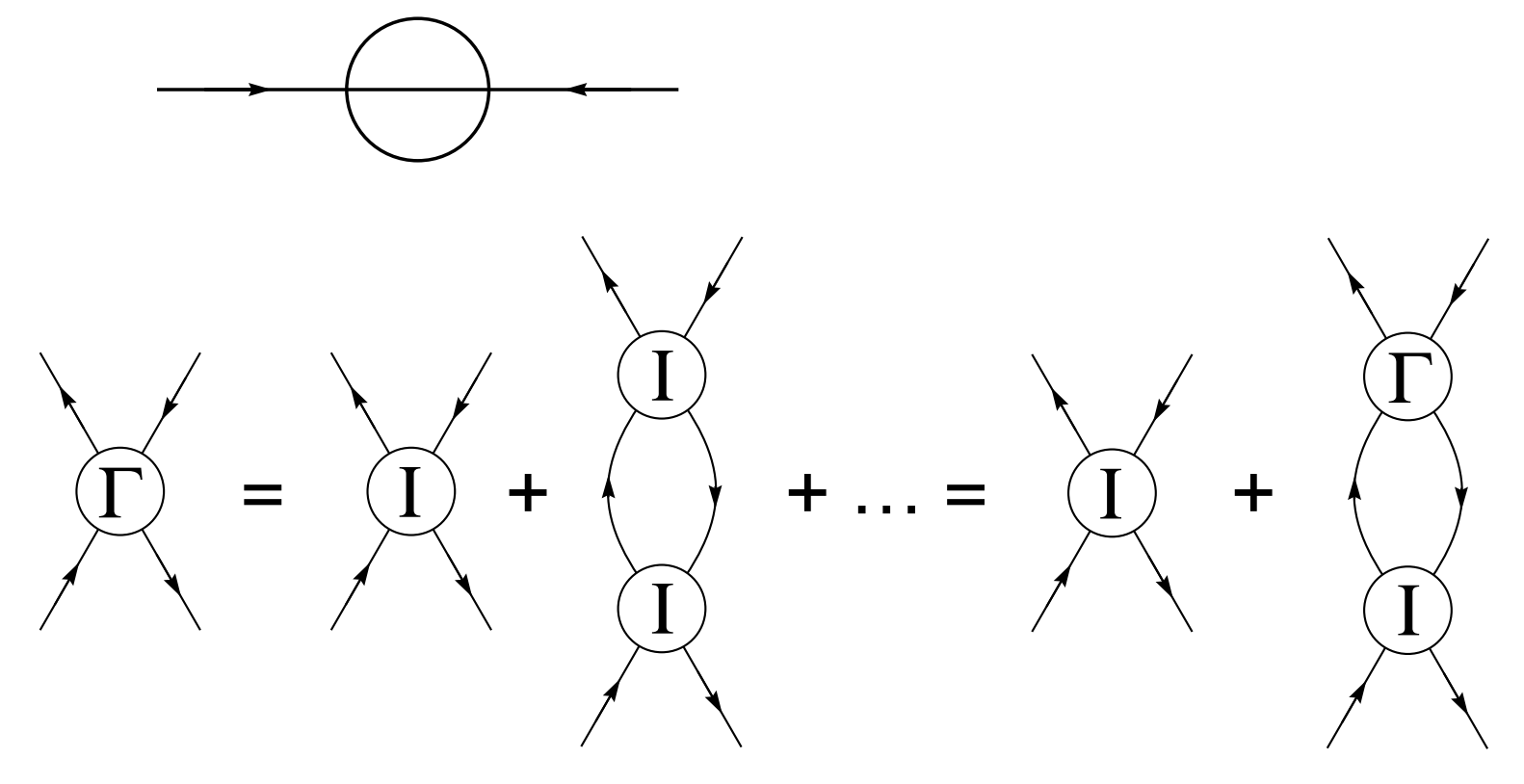
T. T. 


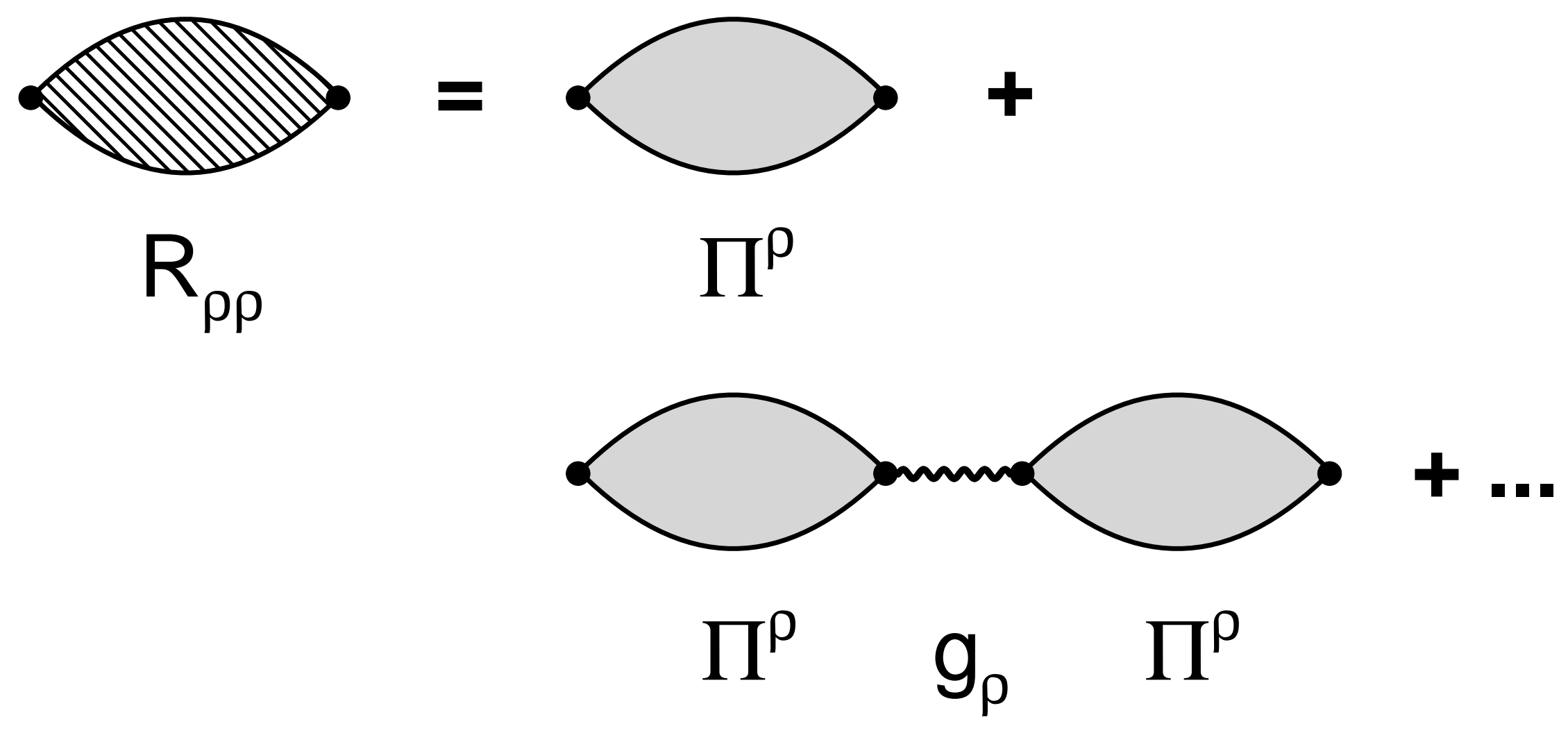




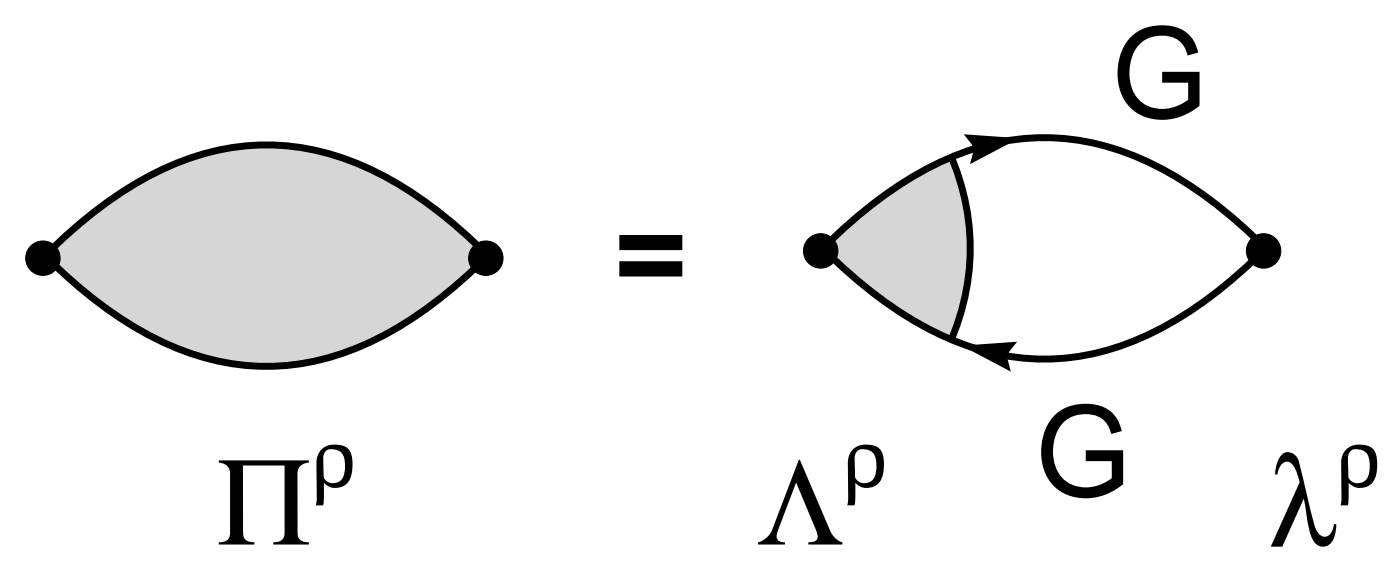




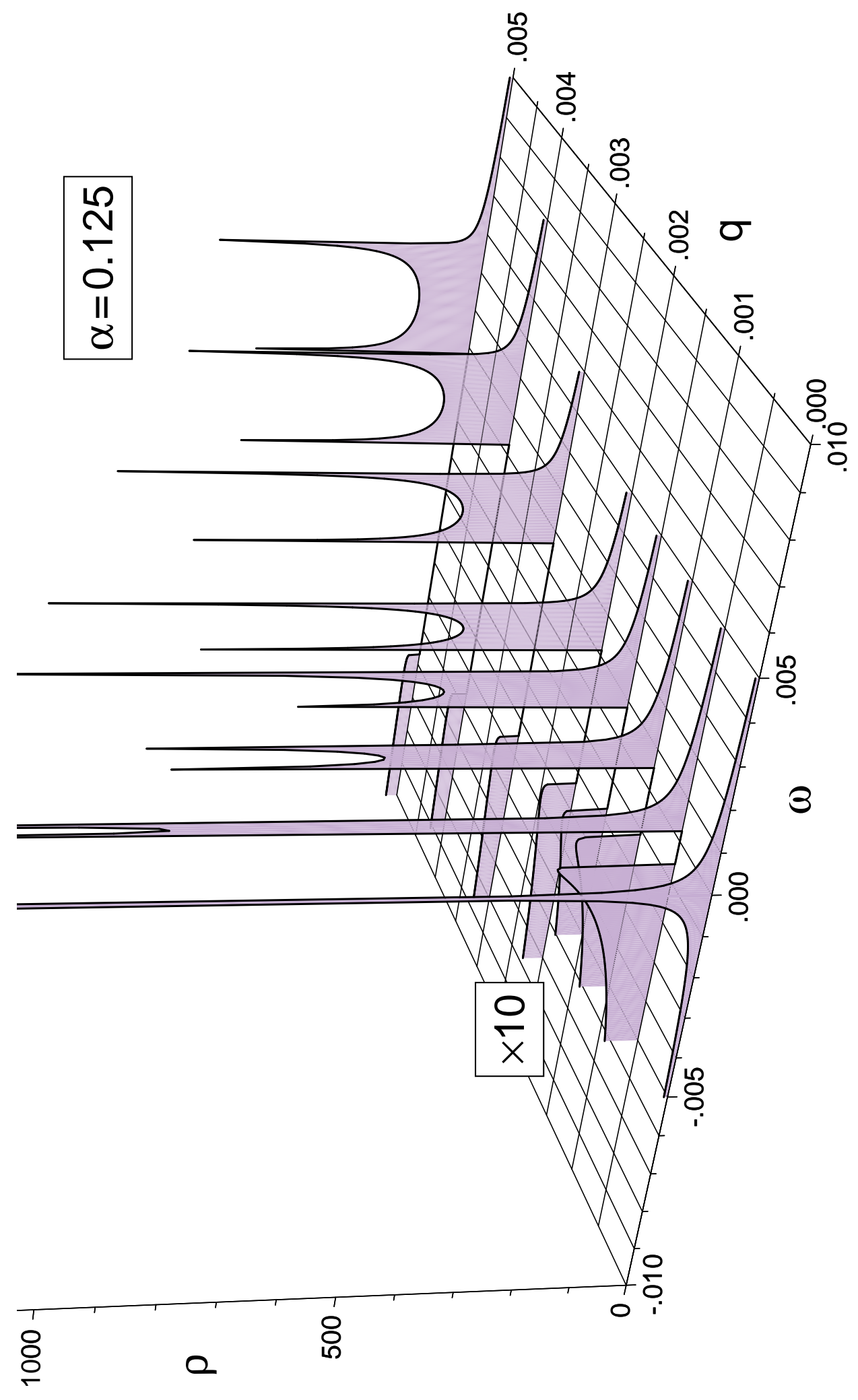



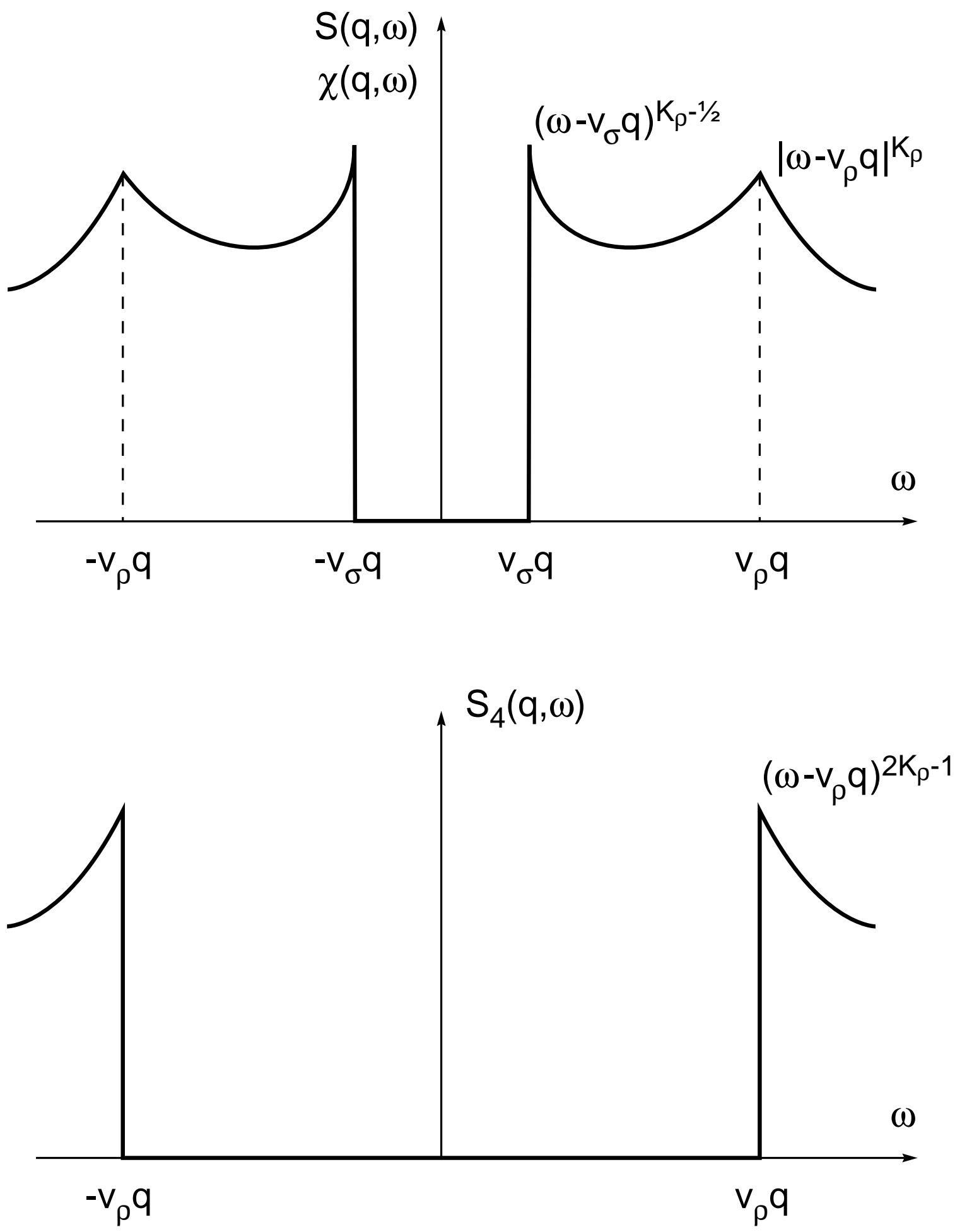